\title{
Liquid Crystals in Aqueous lonic Surfactant Solutions: Interfacial Instabilities \& Optical Applications
}
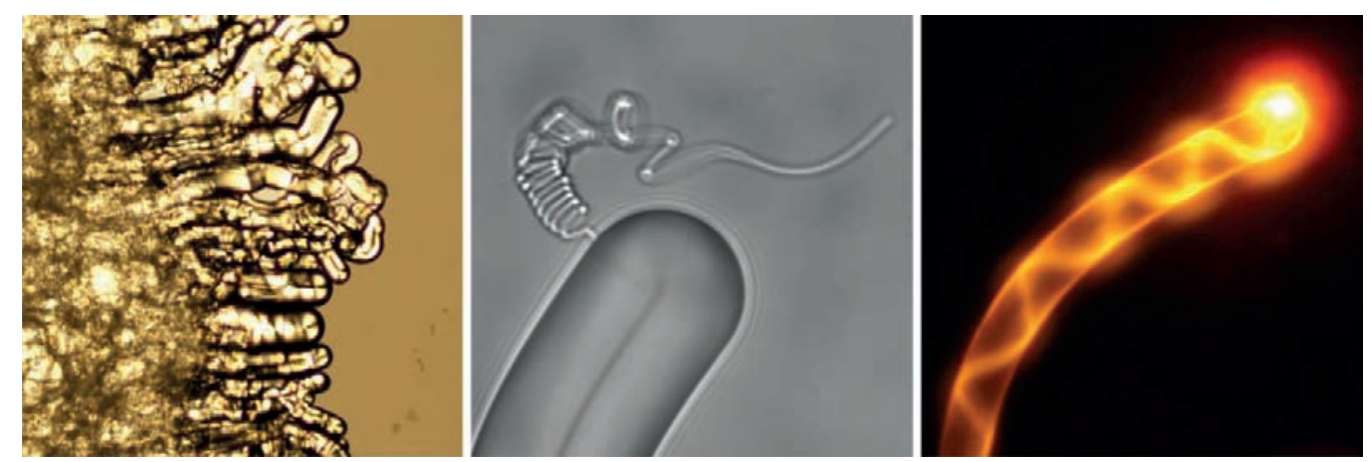

Doctoral Dissertation

Karthik Reddy Peddireddy

Faculty of Physics

University of Göttingen 



\title{
Liquid Crystals in Aqueous Ionic Surfactant Solutions: Interfacial Instabilities \& Optical Applications
}

\section{Dissertation}

zur Erlangung des mathematisch-naturwissenschaftlichen Doktorgrades

"Doctor rerum naturalium" der Georg-August-Universität Göttingen

im Promotionsprogramm ProPhys der Georg-August University School of Science (GAUSS)

\author{
vorgelegt von \\ Karthik Reddy, Peddireddy
}

aus Nagarkurnool, Telangana, India

Göttingen, 2014 


\section{Betreuungsausschuss:}

Prof. Dr. Marcus Müller, Institute for Theoretical Physics, University of Göttingen

Prof. Dr. Stephan Herminghaus, Dynamics of Complex Fluids, Max Planck Institute for Dynamics and Self-Organization, Göttingen

Prof. Dr. Sarah Köster, Institute of X-Ray Physics, University of Göttingen

\section{Mitglieder der Prüfungskommission:}

Referent: Prof. Dr. Marcus Müller, Institute for Theoretical Physics, University of Göttingen

Korreferent: Prof. Dr. Stephan Herminghaus, Dynamics of Complex Fluids, Max Planck Institute for Dynamics and Self-Organization, Göttingen

\section{Weitere Mitglieder der Prüfungskommission:}

Dr. Christian Bahr, Dynamics of Complex Fluids, Max Planck Institute for Dynamics and Self-Organization, Göttingen

Prof. Dr. Jörg Enderlein, Third Institute of Physics, University of Göttingen

Dr. Simone Techert, Structural Dynamics of (Bio)chemical Systems, Max Planck Institute for Biophysical Chemistry, Göttingen

Prof. Dr. Sarah Köster, Institute of X-Ray Physics, University of Göttingen

Tag der mündlichen Prüfung: 12-05-2014 




\section{Acknowledgements}

First of all, I would like to acknowledge financial support from the European Union (EC Marie Curie ITN project Hierarchy-PITN-CA-2008-215851) and Prof. Stephan Herminghaus. I would like to convey my regards to all the members of our Marie Curie network (Hierarchical Assembly in Controllable Matrices) and its coordinator Dr. P.H.J. Kouwer. Through this network, I made many friends (Dr. Venkata Jampani, Dr. Matjaž Humar, David Seč, Dr. Giorgio Mirri, Boldizsár Kurilla, Dr. Uroš Tkalec, Dr. Miha Ravnik, Regina Jose, Maryam Nikkhou and Firat Ozdemir) for which I am thankful to all the masterminds behind this project.

Special thanks to my group leader, Dr. Christian Bahr, who always made himself available for his students almost every working day. He continued to support me despite many failures during the early days of my doctoral studies. I will be eternally grateful to Christian for introducing me to the beautiful world of liquid crystals. I would also like to thank Christian for proof-reading my thesis and translating my abstract into German.

I do not think I could have survived in Germany without the support from my colleagues Dr. Xunda Feng, Dr. Songchuan Zhao, Dr. Shashi Thutupalli, Dr. Sravanti Uppaluri, Sayhwa Tan, Dr. Junaid Laskar, Dr. Marta Sanchez de La lama, Dr. Daniel Herde and Dr. Eric Stellamanns. It is my best friends Xunda and Jampani who encouraged and supported me during the tough times. Xunda and I finished the credits required for our doctoral degrees together and shared ideas almost every day. It is the overflowing enthusiasm of Dr. Jampani for research that kept me going for all these days. The members of the liquid crystal group, Dr. Pramoda Kumar, Dr. Benzamin Schulz, and Dr. Anupam Sengupta, contributed significantly to my work in one way or another. I cannot thank enough the support staff of our Dynamics of Complex Fluids (DCF) department (Ms. Monica Tueteberg, Dr. Kristian Hantke, Markus Benderoth, Wolf Keiderling, Thomas Eggers, Sibylle Nägle, Guido Schriever, Trümper and the late Udo Krafft). Dr. Hantke was always available to share his knowledge in the field of optics. Dr. Lucas Goehring was kind enough to accept our request to conduct SANS experiments on our samples. I am deeply grateful to Dr. Florian Rehfeldt and his team (Christina Jayachandran and Meenakshi Prabhune) for allowing as well as assisting me to use their rheometer. I am also grateful to the support provided by my thesis committee members (Prof. Marcus Müller and Prof. Sarah Köster).

Prof. Herminghaus's visit to the Jožef Stefan Institute in 2012 (his talk: "Experiments with Active Emulsions") acted as a catalyst for my doctoral research. This talk was the initiating point of my collaboration with Prof. Igor Muševič. Prof. Igor kindly invited me to his soft matter laboratory several times and provided excellent scientific ideas. I started appreciating the beauty of the liquid crystal world only after visiting Prof. Igor's lab. I developed a great rapport with the members of his lab (Dr. Miha Škarabot, Dr. Jampani, Dr. Yuji Sasaki-san, Dr. Matjaž, Gregor Posnjak, Huan Peng, Dr. Andriy Nych, Dr. Uliana Ognysta, Dr. Anna Ryzhkova, Nikkhou). I would also like to extend my regards to Prof.

Slobodan Žumer and his team. I will not forget the fun I had during several after-work 
parties in the soft matter lab.

Life is not complete without friends from outside work. I was fortunate enough to make many great friends during my stay in Göttingen (Dr. Bhaskar Bandarapu, Mrs. Radhika Bandarapu, Dr. Raees Ahmed (my mentor), Florian Winkel, Dr. Pitchaiah Cherukuri, Sai srinivas, Sakya Dasgupta, Heike Kristin Paesel (my dance partner), Dr. Shweta Aggarwal, Dr. Harish Potukuchi, Dr. Raghavendran LakshmiNarayanan, Dr. Nivethida Thirugnanasambandam, Toni Walther, Tathagata Banerjee, Dr. Elango Kandasamy, Arun Joseph, Liza Swain, Alana Poloni, Theresa Wollenberg, Nunna Suneetha, Dr. Gopala Darbha, Carolina Ordieres Franky, Andres, Maximilian Puelma Touzel, David Hofmann, Mirko Lukovic, Amir Asgharsharghi and the members of Goettingen University Cricket Club (GCC-CTdUG)).

Finally, I would like to sincerely thank my parents Thirupathi Reddy and Hymavati, and my sister Shrutha Keerthi for their lifetime support. I dedicate this thesis to all the above people. 


\section{Abstract}

This thesis presents the properties and applications of various liquid crystal (LC)-aqueous ionic surfactant systems. It begins with the study of micellar solubilization of different LCs in aqueous ionic micellar solutions. The analysis of a few hundred $\mu \mathrm{m}$-sized LC droplets in either nematic or isotropic state (parent droplets) shows a linear decrease of the droplet size over time. During the entire micellar solubilization process, the material transport across a parent droplet's surface exists in both directions. Numerous aqueous droplets (daughter droplets) start to appear in the parent droplet due to the transport of surrounding aqueous solution into it. The average size of the aqueous daughter droplets and their number density increase over time. But, no daughter LC droplets form in the surrounding aqueous solution due to the transport of LC molecules from the parent LC droplet into it. However, in special cases, depending on the interfacial tension between the parent droplet and its surrounding aqueous solution, the parent droplet may either expel tiny droplets into the surrounding aqueous medium or transform itself into a filament like structure. Furthermore, the material transfer across a parent droplet's surface generates convective flows around it. In concentrated aqueous surfactant solutions, the convective flows are strong enough to propel the parent droplet itself. The parent LC droplets become completely solubilized and a clear aqueous solution is produced, provided the weight fraction of the LC in the system is not larger than a few percent. The properties of the equilibrium LC-aqueous systems are investigated through shear and small angle neutron scattering experiments.

In the second part, the behavior of the smectic droplets in the aqueous micellar solution is presented. A smectic droplet spontaneously produces smectic filaments at the interface with an aqueous solution, which resemble the myelin figures observed in lyotropic lamellar systems. The three-dimensional structure of the smectic-A filaments is studied with the polarizing optical microscopy and the fluorescence confocal polarizing microscopy. The results show that the LC molecules are oriented perpendicular to the surface of the filament and the layers are wrapped around a central disclination line in a concentric cylindrical fashion. Further, the study on the growth and the stability of the smectic-A filaments shows that the freely floating smectic-A filaments are only transient structures and they either transform into discs or break into small smectic droplets. However, it is possible to stabilize the filaments at least for several months, either by confining them in square capillaries or by photo polymerization.

In the last part, the optical applications of the smectic filaments are discussed. In the case of the smectic-A filaments, light guiding through the filaments and Whispering Gallery Mode (WGM) lasing in a plane perpendicular to the filaments are presented. Moreover, it is also shown that smectic-C* filaments act as photonic band-gap waveguides.

Keywords: liquid crystals, surfactants, liquid crystal emulsions, micellar solubilization, artificial swimmers, filaments, myelin figures, liquid crystal ropes, waveguiding, lasing.

PACS: 61.30.Jf, 61.30.-v, 42.79.Gn, 64.75.Yz, 42.82.-m, 42.79.-e, 64.70.pv, 61.30.Hn 


\section{Kurzzusammenfassung}

Die vorliegende Arbeit beschreibt Eigenschaften und Anwendungen von thermotropen Flüssigkristallen in wässrigen Tensidlösungen. Zunächst wird der Prozess der mizellaren Solubilisierung von Flüssigkristalltropfen in wässrigen mizellaren Lösungen ionischer Tenside betrachtet. Der Durchmesser der Flüssigkristalltropfen, der zunächst einige Hundert $\mu \mathrm{m}$ beträgt, nimmt linear mit der Zeit ab; dies wird sowohl für nematische als auch für isotrope Tropfen beobachtet. Zusätzlich zum Transport der Flüssigkristallmoleküle in die Mizellen der wässrigen Umgebung findet auch ein Materialtransport von der wässrigen Phase in die Flüssigkristalltropfen hinein statt, erkennbar an $\mu \mathrm{m}$-großen wässrigen Tröpfchen, die in den Flüssigkristalltropfen erscheinen. In einigen Fällen wird auch beobachtet, dass der Flüssigkristalltropfen kleinere, $\mu$ m-große Tröpfchen oder filamentartige Strukturen in die wässrige Umgebung abgibt, zusätzlich zum molekularen Transport in die Tensidmizellen. Der Solubilisierungsprozess der Flüssigkristalltropfen induziert konvektive Strömungen in den Tropfen und der umgebenden wässrigen Phase, die zu einer selbständigen Fortbewegung der Flüssigkristalltropfen führt. Wenn der Gewichtsanteil des flüssigkristallinen Materials in der wässrigen Phase nicht mehr als wenige Prozent beträgt, werden die Flüssigkristalltropfen vollständig solubilisiert und es kommt schließlich zur Bildung einer Mikroemulsion, die durch rheologische Messungen und Kleinwinkelneutronenstreuung untersucht wurde.

Der zweite Teil der Arbeit beschäftigt sich mit dem Verhalten von smektischen Flüssigkristalltropfen in wässrigen mizellaren Tensidlösungen. Während der Solubilisierung bilden smektische Tropfen spontan filamentartige Strukturen, ähnlich den Myelinfiguren in lyotropen Systemen, an der Grenzfläche zur wässrigen Phase aus. Untersuchungen mit dem Polarisationsmikroskop und dem konfokalen Fluoreszenzpolarisationsmikroskop zeigen, dass in den smektischen Filamenten die molekularen Schichten eine konzentrische zylindrische Anordnung um eine zentrale Defektlinie entlang der Filamentachse ausbilden. Mit fortschreitender Solubilisierung bilden sich an der Oberfläche der zuerst entstandenen Filamente sekundäre dünnere Filamente aus, die schließlich in kleine Tröpfchen oder andere Fragmente zerfallen und letztlich vollständig solubilisiert werden. In eingrenzender Umgebung, z. B. in Kapillaren, oder durch Photopolymerisation lassen sich die smektischen Filamente zumindest für mehrere Monate stabilisieren.

Schließlich werden optische Anwendungen der smektischen Filamente diskutiert. Filamente der smektischen A Phase lassen sich als optische Wellenleiter einsetzen. Auch die Induzierung von Laseraktivität, basierend auf Whispering-Gallery-Moden, wird demonstriert. Filamente der chiralen smektischen $\mathrm{C}^{*}$ Phase besitzen eine periodisch modulierte Ortsabhängigkeit des Brechungsindex und weisen daher auch eine photonische Bandlücke auf, die die optische Wellenleitung beeinflusst. 


\section{Contents}

Acknowledgements $\quad$ V

$\begin{array}{lll}\text { Abstract } & \text { VII }\end{array}$

$\begin{array}{lll}\text { Kurzzusammenfassung } & \text { VIII }\end{array}$

1 Introduction 1

1.1 Thermotropic LC phases . . . . . . . . . . . . . . . . . . . . . . . . 3

1.2 Lyotropic LC phases . . . . . . . . . . . . . . . . . . . . . . . . 6

1.3 Thesis outline . . . . . . . . . . . . . . . . . . 10

2 Materials and methods $\quad 11$

2.1 Materials . . . . . . . . . . . . . . . . . . 11

2.2 Experimental methods . . . . . . . . . . . . . . . . . . . 13

2.2.1 Characterization of the micellar solutions . . . . . . . . . . . 13

2.2.2 Polarizing optical microscopy . . . . . . . . . . . . . . . . . 14

2.2.3 Fluorescence confocal polarizing microscopy . . . . . . . . . . . . . . 15

2.2.4 Optical setup for waveguiding experiments . . . . . . . . . . 17

2.2.5 Optical setup for lasing experiments . . . . . . . . . . . . . . 19

3 Results and discussion $\quad \mathbf{2 1}$

3.1 Nematic liquid crystals in aqueous ionic micellar solutions . . . . . . . . . . 21

3.1.1 Phase diagrams: Equilibrium states of NLCs in cationic micellar so-

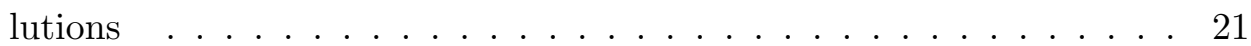

3.1.2 Microscopy observations: Solubilization of NLCs in TTAB solutions . 24

$3.1 .35 \mathrm{CB}$ droplets . . . . . . . . . . . . . . . . . 25

3.1.3.1 Solubilization of 5CB vs Temperature . . . . . . . . . 27

3.1.3.2 Solubilization of $5 \mathrm{CB}$ vs TTAB concentration . . . . . . 28

3.1.3.3 Effect of solubilizate chain length on solubilization rates . . 29

3.1 .4 MBBA droplets . . . . . . . . . . . . . . . . . . 30

3.1.4.1 Spontaneous expulsion of daughter MBBA droplets - Threshold temperature $\left(T_{c}\right) \ldots \ldots \ldots \ldots$

3.1.4.2 Solubilization of MBBA vs Temperature . . . . . . . . . 32

3.1.4.3 Solubilization of MBBA vs TTAB concentration . . . . . . . 33

3.1.5 Plausible molecular pathways in the micellar solubilization of LC droplets

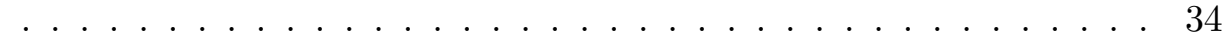

3.1.5.1 Todorov's theoretical model . . . . . . . . . . . . . . 36

3.1.6 Rheology of LCs in TTAB solutions at equilibrium . . . . . . . . 38

3.1.6.1 5CB in TTAB 25 wt\% solutions: Steady shear flow curves . 39 
3.1.6.2 nCB compounds in TTAB 25 wt\% solutions: Effect of added solubilizate chain length on steady shear flow properties . . 43

3.1.6.3 5CB in TTAB $25 \mathrm{wt} \%$ solutions: Oscillatory shear measure-

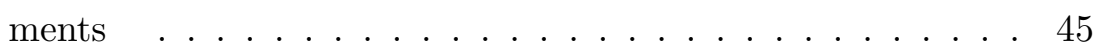

3.1.6.4 MBBA in TTAB 25 wt\% solutions: Steady shear flow curves 45

3.1.7 5CB in TTAB 25 wt\% solution: Small-angle neutron scattering (SANS)

3.1.8 5CB droplets in aqueous ionic micellar solutions: Self-propelled motion . . . . . . . . . . . . . . . . . . . 49

3.1 .9 Nematic filaments . . . . . . . . . . . . . . . . . . 53

3.1.9.1 Reversible transformation: Isotropic droplet $\leftrightarrow$ nematic fil-

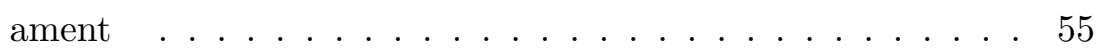

3.2 Smectic liquid crystals in aqueous ionic miceller solutions . . . . . . . . . . . 61

3.2.1 Lyotropic lamellar liquid crystals: Myelin figures . . . . . . . . . . 61

3.2.2 Three dimensional structure of the smectic filaments . . . . . . . . 63

3.2.3 Growth behavior of thermotropic smectic-A filaments . . . . . . . 75

3.2.3.1 Sandwich method . . . . . . . . . . . . . 75

3.2.3.2 Growth rates of smectic-A filaments: Effect of CTAB con-

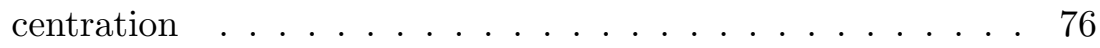

3.2.3.3 Growth rates of smectic-A filaments: Effect of temperature $\quad 77$

3.2.3.4 Effect of the confining walls on the morphology of smectic-A filaments ................... 78

3.2.3.5 Growth rates of smectic-A filaments: Surfactant chain length dependence . . . . . . . . . . . . 78

3.2.3.6 Capillary method . . . . . . . . . . . . . . . . 79

3.2.3.7 Confined single filaments: Effect of the capillary dimensions and the surfactant concentration . . . . . . . 80

3.2.3.8 Smectic-A filaments' growth: Comparison between sandwich and capillary methods . . . . . . . . . . . 81

3.2.4 Surface instabilities of smectic filaments: Liquid crystal (LC) ropes $\quad$. 82

3.2.4.1 LC ropes: Why do they form on the surface of smectic filaments? . . . . . . . . . . . . . 83

3.2.4.2 Temperature dependence of the spontaneous ejections of LC

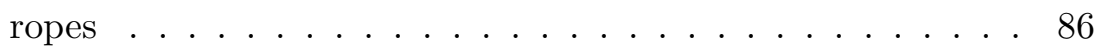

3.2.4.3 LC ropes: Liquid rope coiling effect . . . . . . . . . . . 87

3.2.4.4 LC rope helical coils: Effect of salt in the aqueous phase . . 88

3.2.5 UV photo-polymerization of smectic filaments . . . . . . . . . . 89

3.2.6 Self-propelled motion of smectic droplets . . . . . . . . . . . . . 91

3.2.7 Optical applications of smectic filaments f . . . . . . . . . . . 92

3.2.7.1 Waveguiding in smectic-A filaments . . . . . . . . . . . 93

3.2.7.2 Lasing in smectic-A filaments . . . . . . . . . . . . . 98

3.2.7.3 Optical properties of smectic- $C^{*}$ objects (droplets and filaments $\ldots \ldots \ldots \ldots 1$

4 Conclusions 105

$\begin{array}{llr}5 & \text { References } & 109\end{array}$

$\begin{array}{lr}\text { Index of Figures } & 123\end{array}$ 
Index of Tables

Abbreviations 128

Appendix 


\section{Introduction}

"When an oil (5CB) droplet is placed in an aqueous micellar surfactant (TTAB) solution, it continues to self-propel itself for several days without any need for external energy depending on the parameters of the system (i.e. surfactant concentration, water to oil ratio, temperature and the molecular solubility of the oil in the pure water). For a fleeting moment, it feels like a perpetual motor is achieved. But in reality self-propulsion of the oil droplet is a side effect of its solubilization in the aqueous micellar solution. The oil droplet's motion exists as long as there is a mass-transfer across the oil-aqueous interface. If we artificially create a surfactant concentration gradient in the aqueous solution, oil droplets spontaneously propel themselves towards the region containing the highest surfactant concentration in the system (artificial chemotaxis). This simple three component non-equilibrium system containing organic molecules and water produces an object (oil droplet) with life-like properties".

The interaction between organic molecules and water is a fundamentally important topic to investigate as this may enable us to discover the likely routes of abiogenesis ${ }^{1}$ [1]. Complex organic molecules and water are present ubiquitously throughout the cosmos as shown in Fig.

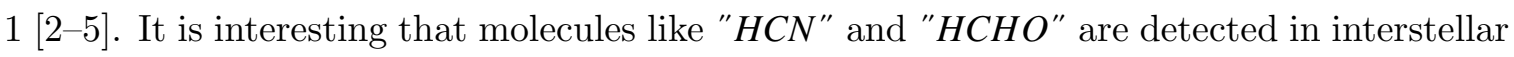
molecular clouds [3]. These two molecules are extremely reactive and they can easily form amino acids. Amino acids are also detected in other objects that belong to our solar system - comets and meteorites [3]. The famous "Miller-Urey experiment" has also shown us that amino acids can be easily produced from a mixture of simple chemicals $\left(\mathrm{CH}_{4}, \mathrm{NH}_{3}, \mathrm{H}_{2}\right.$ and $\mathrm{H}_{2} \mathrm{O}$ ) in a test tube [6-10]. Amino acids combine to form proteins, one of the two kinds of large biomolecules which form the basis of life on earth. Proteins are the workhorse molecules of life, which are required for the structure, function and regulation of everything in our body. The other kind of essential biomolecules are nucleic acids. Nucleic acids store coded instructions which are used by cell machinery for producing proteins. Nucleobases such as adenine and guanine (the components of nucleic acids) are found in meteorites [11]. Just like amino acids, nucleobases are produced from a mixture of $\mathrm{HCN}, \mathrm{NH}_{3}$ and $\mathrm{H}_{2} \mathrm{O}$ in the laboratory. The most basic building block of nucleic acids is a sugar molecule called ribose. This complex sugar molecule can be formed by using much simpler sugar molecules such as glycolaldehyde. Glycolaldehyde is in fact found in the Sagittarius B2, the interstellar molecular cloud which lies near the center of our galaxy [12,13]. Even though building blocks of proteins and nucleic acids are detected in space, it is not yet known how to build these bio-macromolecules from their respective building blocks. Thus, the logical next step is to find out the way by which nature has transformed inanimate organic matter into these bio-macromolecules and eventually into life forms. The resolution of the mystery behind the abiogenesis would not only satisfy our curiosity regarding the purpose of life but would also

\footnotetext{
${ }^{1}$ Abiogenesis is the chemical process through which the simplest life emerges from inanimate matter.
} 

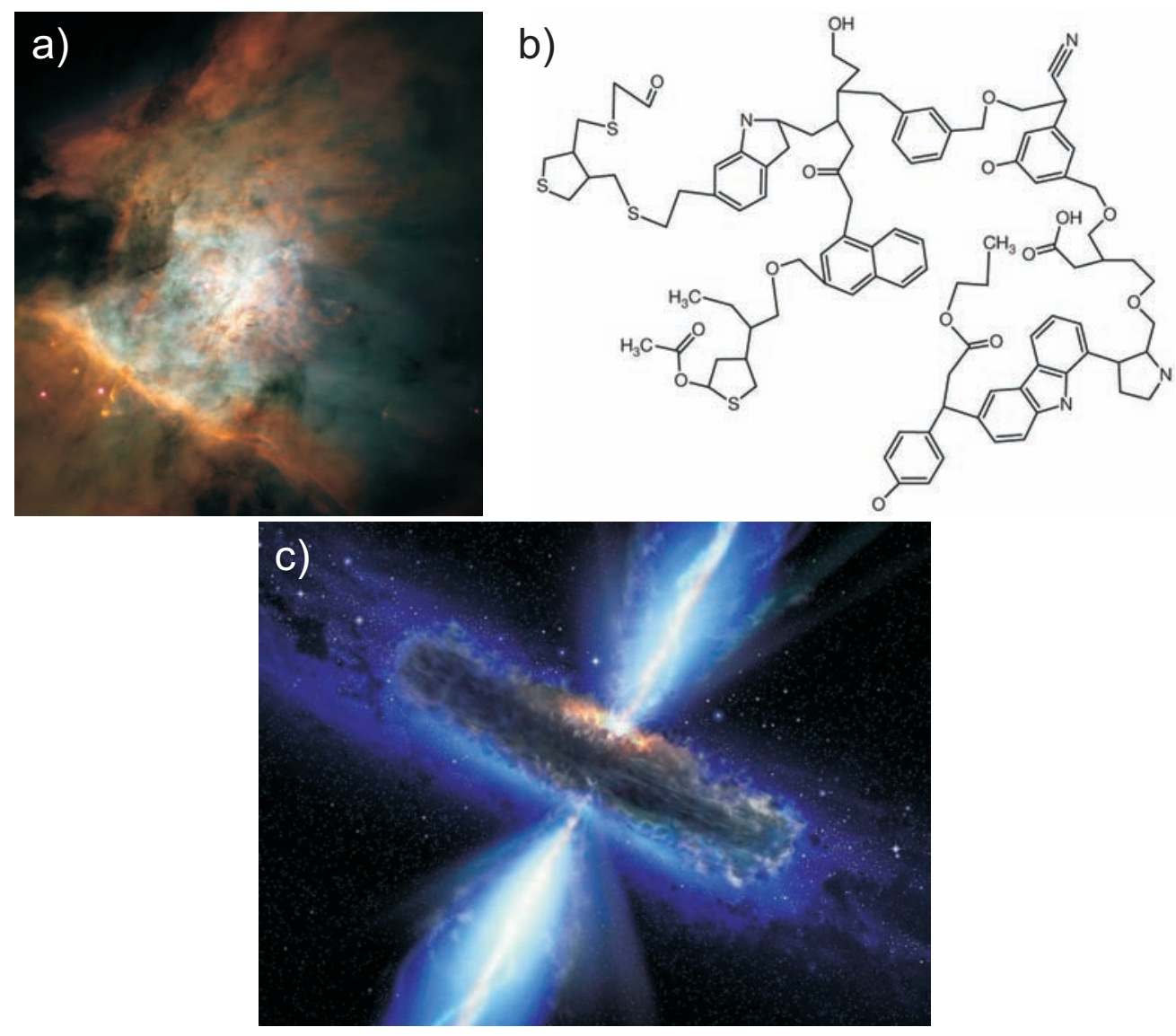

Figure 1: Cosmological structures that contain potentially life-enabling molecules. (a) This picture represents the center of the Orion nebula, a star-forming region located at a distance of 1500 light years away from us in the constellation Orion the Hunter. Numerous complex organic molecules along with water are detected throughout this region of active star formation. Image credit: NASA, C. R. O'Dell and S. K. Wong (Rice University) (b) This complex organic molecule with a mixed aliphatic-aromatic structure is detected in the Orion nebula [4]. It is important to note that this structure is similar to that of the organic compounds found in the famous Murchison meteorite [5]. (c) This is an artistic representation of a quasar. Huge amounts of water vapor (4000 times more water vapor is present than in our galaxy) are detected in the quasar APM 08279+5255 located more than 12 billion light years away from us. Image credit: NASA/ESA.

help us to produce new forms of life. From a practical standpoint as well, the knowledge of interactions between water and organic matter will be extremely useful for commercial emulsions, oil recovery and drug delivery, etc. [14].

Biological molecules in a completely disordered state (isotropic) or a completely rigid state (crystal) cannot support life $[15,16]$. For example, a cell membrane which consists of lipids and proteins must be fluid enough to selectively allow material across the membrane but it should also be rigid enough to maintain its structure. This intermediate state that exists in the cell membrane is called the liquid crystalline state (lamellar). Loss of the liquid crystalline (LC) state within the cell membrane results in death. For any given mixture of biological molecules, the liquid crystalline state exists at a range of temperatures and pressures. Thus, living organisms can only survive within certain ranges of temperatures and pressures and, the habitable environment for any living organism is directly dependent on its composition of organic matter and water. This makes it necessary to investigate the various liquid crystalline phases formed by organic matter and water. 


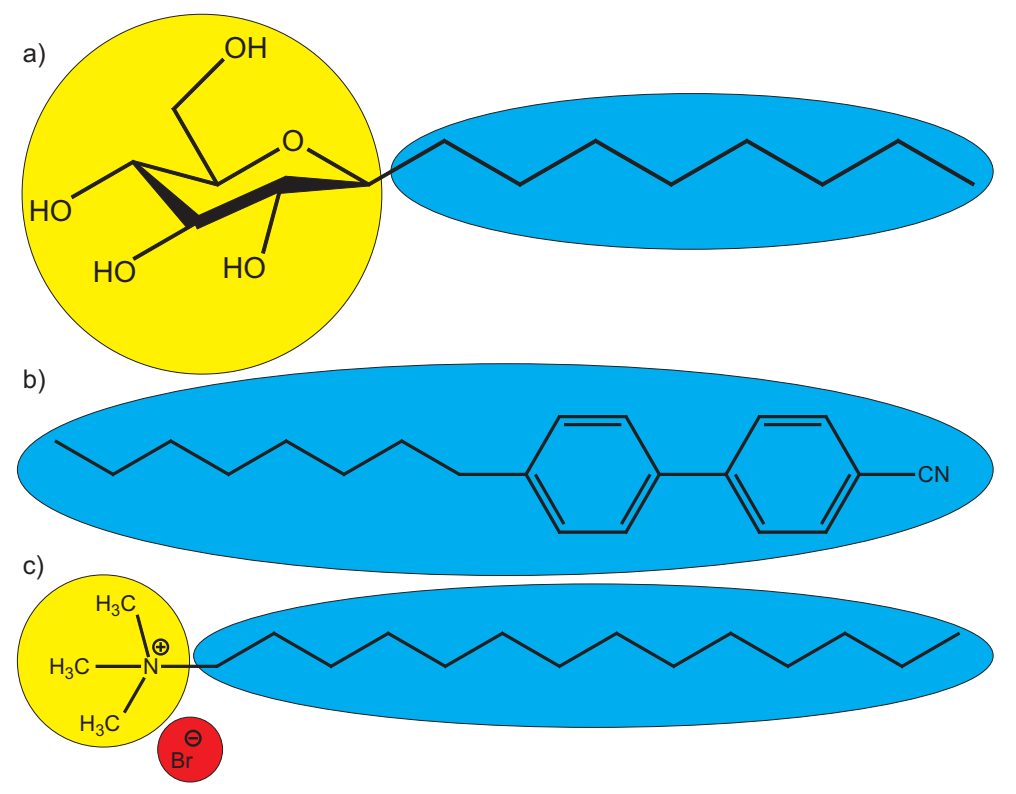

Figure 2: Molecular structures of typical organic molecules that form LC phases. Yellow and blue circles indicate hydrophilic and hydrophobic regions respectively. The red circle represents a counter ion. (a) Octyl $1-O-\beta$-D-glucopyranoside forms both lyotropic and thermotropic LC phases [15]. (b) 4'-n-octyl-4-cyanobiphenyl (8CB) forms only thermotropic LC phases as it is sparingly soluble in water $(5 \mu \mathrm{g} / \mathrm{L})$. (c) Tetradecyltrimethylammonium bromide (TTAB) can only form lyotropic LC phases.

Many organic molecules like glycolipids are amphiphilic in nature (Fig. 2(a)). This means that they contain both water-loving (hydrophilic) and water-averse (hydrophobic) regions. The LC phases formed by these organic molecules on their own are called thermotropic LC phases and the LC phases formed by mixing them in water are called lyotropic LC phases. Some organic molecules like 4'-n-alkyl-4-cyanobiphenyls (Fig. 2(b)) can only form thermotropic LC phases where as many commercial surfactants (Fig. 2(c)) form lyotropic LC phases exclusively.

\subsection{Thermotropic LC phases}

Thermotropic LC phases were first identified by an Austrian botanist, Freidrich Reinitzer in 1888 [17]. He observed that a substance related to cholesterol (cholesteryl benzoate) has two melting points. On heating the solid form of this compound, it first turns into a turbid liquid at $145.5^{\circ} \mathrm{C}$ and then turns into a clear liquid at $178.5^{\circ} \mathrm{C}$. The thermal behavior of this compound is reversible, as he observed the same transition temperatures while cooling the clear liquid back into the solid form. He subsequently collaborated with a German physicist, Otto Lehmann, who found that this compound in its turbid liquid phase shows both fluid and crystal properties at the same time. For example, this turbid liquid flows like a fluid but it also exhibits optical birefringence which was at that time thought to be the virtue of anisotropic crystals only. Thus, Lehmann began to call the organic compounds which show multiple melting points "liquid crystals". In 1922, Georges Friedel classified the turbid liquid phases observed in various organic compounds with multiple melting points (i.e., liquid crystals) into three categories based on internal molecular arrangements [18]. These three phases are named as nematic, smectic and cholesteric phases. Any organic compound (or a mixture of organic compounds) which exhibits one or more of these three 
phases in certain temperature ranges is more specifically called a thermotropic liquid crystal.

Not all organic molecules can form thermotropic LC phases. For example, hexane can only exist in three standard states of matter - solid, isotropic liquid and gas. The hexane molecules in the isotropic liquid phase diffuse randomly without any orientational or positional order, whereas they directly attain both positional and orientational order spontaneously upon cooling below the melting temperature $\left(-96\right.$ to $\left.-94^{\circ} \mathrm{C}\right)$. This means hexane cannot form any LC phase between the isotropic liquid phase and the solid phase. The molecular structure of organic molecules determines whether they exhibit thermotropic LC phases. They must have molecular shape anisotropy, which means that one molecular axis is considerably different from the other two axes. As shown in Fig. 3, thermotropic liquid crystals can be classified into two types based on their molecular shapes - calamitics (rod-like) and discotics (disc-like). Thermotropic LCs must have rigid cores in order to maintain their anisotropic molecular shapes. Rigid cores can be achieved by using two or more ring structures, linked together with or without unsaturated linking groups. Rigid cores are always attached by one or more flexible hydrocarbon chains such as alkyl or alkyloxy chains. It is generally not possible to predict the thermotropic LC phases formed by any organic molecule just by knowing its molecular structure. Since my work is undertaken using calamitic liquid crystals exclusively, thermotropic LC phases exhibited by disc-like molecules are not included in this discussion.
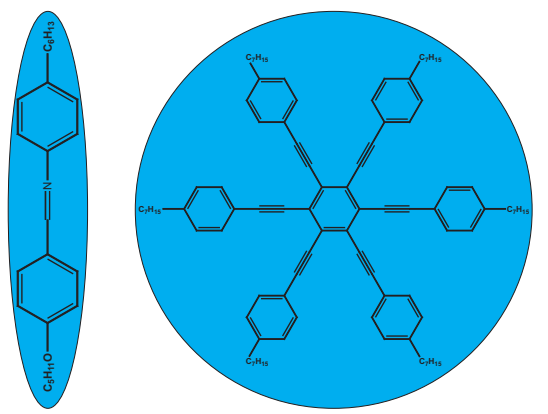

Figure 3: Schematic representation of typical calamitic and discotic LC molecules.

The nematic phase $(\mathrm{N})$ produced by calamitic molecules is the most common and simplest thermotropic LC phase with a long-range orientational order. In this phase, the molecules diffuse much like the molecules in an isotropic liquid phase but their long molecular axes always tend to point along a preferred direction (Fig. 4). This preferred direction is known as the director and it is generally represented with the unit vector, $\vec{n}$. It is generally observed that $\vec{n} \equiv-\vec{n}$, which means that the nematic phase does not show polar order. An order parameter, $\mathrm{S}$, is introduced to quantify the extent of the orientational order in the nematic phase. $\mathrm{S}$ is defined as the average of the second Legendre polynomial,

$$
S=\frac{1}{2}<3 \cos ^{2} \theta-1>,
$$

where $\theta$ represents the angle between the long molecular axis and the director as shown in Fig. 4. The brackets indicate the temporal and the spatial average over an entire sample at any given time. $\mathrm{S}=1$ represents the perfectly oriented nematic LC sample whereas $\mathrm{S}=$ 0 represents the isotropic phase. Under no external forces, typical nematic LCs have order parameter values between 0.3 and 0.8. The $\mathrm{S}$ value for any LC decreases with increasing temperature. The director of a nematic LC can be easily influenced by an external electrical or magnetic field. This property of nematic LCs is successfully exploited to produce liquid crystal displays (LCDs). 


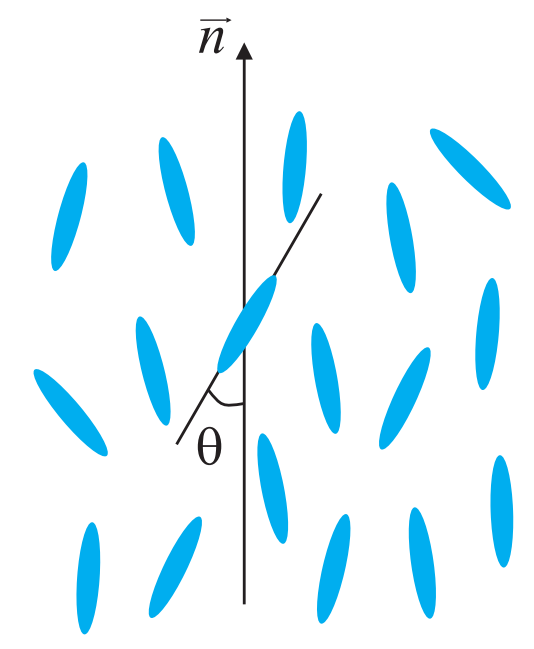

Figure 4: Schematic illustration of the molecular order in a nematic LC.

If we cool a nematic LC, molecules may sometimes attain extra positional order in addition to the existing orientational order. This means molecules are spontaneously arranged in layers as shown in Fig. 5. If the director of the LC sample is parallel to the layer normal, then this particular phase is called a smectic-A LC phase (Fig. 5(a)). If the directions of the director and the layer normal do not match, then it is called a smectic-C LC phase (Fig. $5(\mathrm{~b}))$. In these two phases, there is no further positional order within each layer. Several other higher ordered smectic LC phases (B, E, F, H, I, J and K) have also been discovered. These higher ordered smectic phases are further grouped into two categories: smectic hexatic phases $\left(\mathrm{B}_{\text {hexatic }}, \mathrm{F}\right.$ and $\left.\mathrm{I}\right)$ and crystal mesophases $\left(\mathrm{B}_{\text {crystal }}, \mathrm{H}, \mathrm{I}, \mathrm{J}\right.$ and $\left.\mathrm{K}\right)$. Smectic hexatic phases contain a certain amount of positional order within each layer but no correlation of the positional order exists between adjacent layers (i.e., the positional order in these phases is strictly two-dimensional). On the contrary, crystal mesophases contain three-dimensional positional order but the order does not extend beyond a few layers. It is important to note that some organic materials directly transform into smectic phases from the isotropic phase by skipping the nematic LC phase.

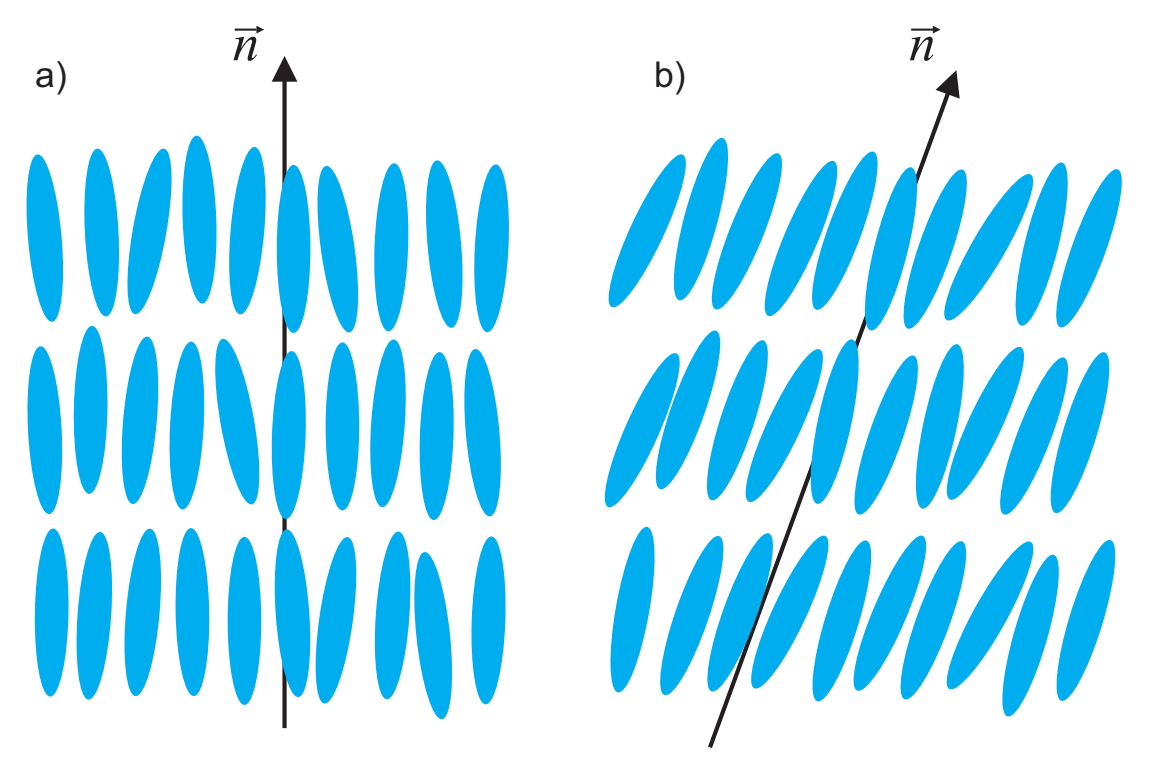

Figure 5: Schematic representation of the thermotropic LC phases with positional order. (a) Smectic-A phase (b) Smectic-C phase. 
If the calamitic molecules have one or more chiral centers, then the chiral versions of the above thermotropic LC phases form ${ }^{2}$. For example, the nematic LC phase is replaced by the chiral nematic (cholesteric, $\mathrm{N}^{*}$ ) phase, in which the director rotates in a helical fashion about an axis perpendicular to the director (Fig. 6(a)). The distance over which the director rotates by $360^{\circ}$ along the helix is known as the pitch of a chiral nematic phase. A chiral nematic phase is also produced by doping a nematic phase formed by achiral molecules with a small amount of a chiral material/dopant. The helical pitch of this chiral nematic phase decreases with increasing concentration of chiral dopant. Both chiral and achiral versions of a smectic-A phase are structurally identical. Fig. 6(b) shows the internal molecular structure that exists in the chiral version of a smectic-C phase (chiral smectic-C, smectic- $\mathrm{C}^{*}$ ). In this phase, the director rotates around a hypothetical cone as the position along the layer normal is varied. If equal parts of each optical isomer of a chiral calamitic compound are mixed together (racemic mixture), predictably, achiral versions of the LC phases form.

a)

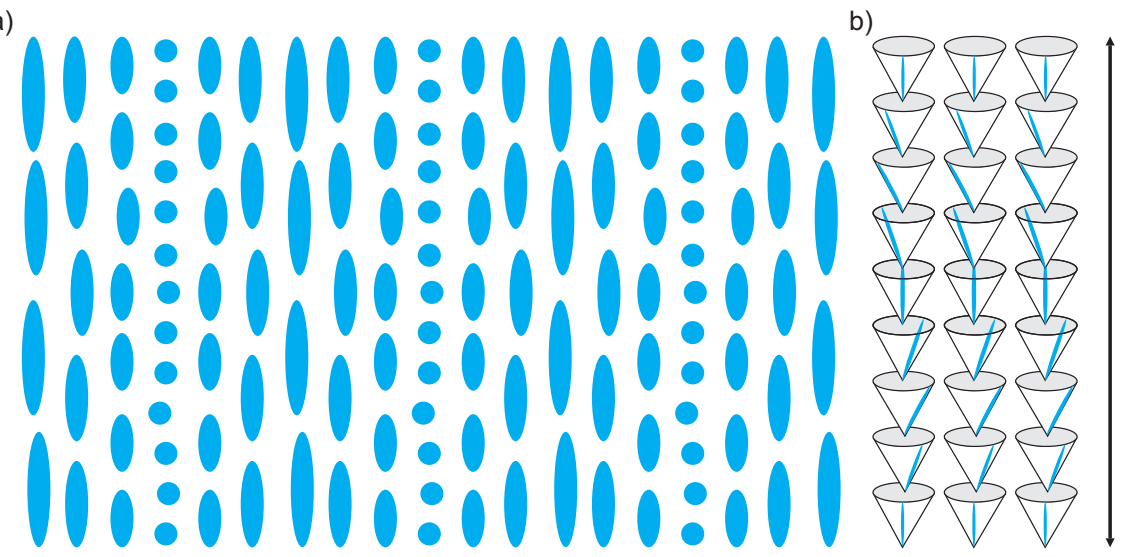

Figure 6: Schematic representation of the thermotropic chiral LC phases. Double-sided black arrows indicate the pitch of the respective LC phases. (a) Chiral nematic phase. (b) Chiral smectic phase. Blue ellipsoids represent an organic material that forms a smectic-C* phase. Hypothetical cones are included to help visualize the orientation of the molecules.

\section{$1.2 \quad$ Lyotropic LC phases}

Mother Nature uses lyotropic LC phases for many purposes. For example, spider silk owes its great mechanical strength to the lyotropic LC phase formed by an aqueous protein solution that is secreted by spider glands [19]. The precise ordering of protein molecules in silk is critical for its outstanding mechanical properties. Similarly many other biopolymers, such as DNA and cellulose, form lyotropic LC phases in aqueous solutions (sometimes also in organic solvents) at high concentrations ${ }^{3}$ [20]. By studying these lyotropic LC phases we may explain the origin of many biological structures as well as find ways to treat diseases such as Parkinson's disease. For example, it is difficult to imagine how a bacterial flagellum (which is made by about 25 different proteins) has evolved over time [21,22]. Creationists use this biological structure as an example to support a theory known as "irreducible complexity"

\footnotetext{
${ }^{2}$ In order to differentiate chiral versions from the thermotropic LC phases formed by achiral calamitic molecules, chiral phases are often designated by an asterisk.

${ }^{3}$ The concentrations at which DNA forms LC phases are very much comparable to those observed in vivo. It is well known that DNA concentration in chromosomes can reach up to $800 \mathrm{~g} / \mathrm{L}$. Lyotropic LC phases like cholesteric and blue phases can be formed by DNA well below this concentration.
} 
[23]. This term was coined by a biochemistry professor Micheal Behe who defined it as: "A single system which is composed of several interacting parts that contribute to the basic function, and where the removal of any one of the parts causes the system to effectively cease functioning". But this apparently complicated tail- or whip-like structure might have had a very simple lyotropic LC phase origin. The possible ways of the origin can be visualized if we examine the behavior of LC-aqueous interfaces. For example, spectacular single- and double-stranded filaments form at a thermotropic cholesteric LC-aqueous interface under the right conditions (i.e., ultra-low interfacial tension), as shown in Fig. 7.

Apart from the above mentioned fairly rigid lyotropic LC polymers, simple flexible organic molecules can also form several different lyotropic LC phases. In order to form LC phases, these flexible organic molecules must be amphiphilic in nature, possessing both polar and non-polar regions (Fig. 2(c)). Up to a certain concentration of an amphiphile, which is known as the critical micellar concentration $(\mathrm{CMC})$, they dissolve in water ${ }^{4}$ on a molecular level (monomer state) and no molecular aggregates are formed. The hydrophobic interactions between amphiphilic molecules lead to the formation of molecular aggregates. In order to form molecular aggregates, the temperature of the aqueous solution must also be above a limit known as the Krafft temperature. The Krafft temperature increases with increase in the hydrophobicity of the amphiphile. Below the Krafft temperature and above the CMC of an amphiphile, hydrated crystals of the amphiphile co-exist with an aqueous solution containing the amphiphile monomers. Above the Krafft temperature and above the CMC of an amphiphile, several types of molecular aggregates - including lyotropic LC phases - form, depending on the amphiphile-to-water ratio. A typical phase diagram of a two component water-amphiphile system is shown in Fig. 8(a). Generally, near-spherical micellar aggregates form just above the CMC (Fig. 8(b-c)). At these dilute concentrations, micelles have no particular orientational or positional order in the aqueous solution. Thus, the resulting aqueous micellar solution $\left(L_{1}\right)$ does not exhibit any birefringence under a polarizing microscope and this micellar phase is not considered a lyotropic LC phase. The simplest lyotropic LC phase is formed when these micelles are arranged in a structure similar to a face-centered or bodycentered cubic crystal lattice (Fig. 8(d)). This phase is known as a micellar cubic phase $\left(I_{1}\right)$ and it also does not exhibit birefringence. Increasing the amphiphile concentration further, the shape of the micelles becomes distorted and forms different shapes like ellipsoids and cylinders (Fig. 8(e)). These anisotropic micelles sometimes form a nematic phase ${ }^{5}$ before a more ordered and viscous hexagonal lyotropic LC phase $\left(H_{1}\right)$ forms. The hexagonal phase is produced by cylindrical micelles of indefinite length arranged in a hexagonal manner (Fig. 8(f)). The lamellar lyotropic LC phase $\left(L_{\alpha}\right)$ forms at amphiphile concentrations above $50 \mathrm{wt} \%$. As shown in Fig. 8(g), this phase consists of a layered arrangement of amphiphiles. Each amphiphile layer is essentially a bilayer of oppositely-directed amphiphiles with hydrophilic regions exposed to water. These bilayer amphiphile sheets are separated by layers of water. Due to this layered structure, lamellar phases are less viscous than hexagonal phases, despite forming at relatively higher amphiphile concentrations. Many more lyotropic LC phases are observed in various amphiphile-water systems but they are omitted here $[26-28]$.

Amphiphiles are generally used to reduce the dissimilarity (interfacial or surface tension)

\footnotetext{
${ }^{4}$ Based on solute-solvent interactions, molecular aggregates can be formed in any type of solvent. For example, commercial surfactants form lyotropic LC phases in glycerol as well as in water [25]. But as this thesis is focused on the interactions between organic molecules and water, discussion is restricted to aqueous solutions.

${ }^{5}$ Predictably, a chiral version of the amphiphile produces a chiral nematic phase instead of a general nematic phase.
} 

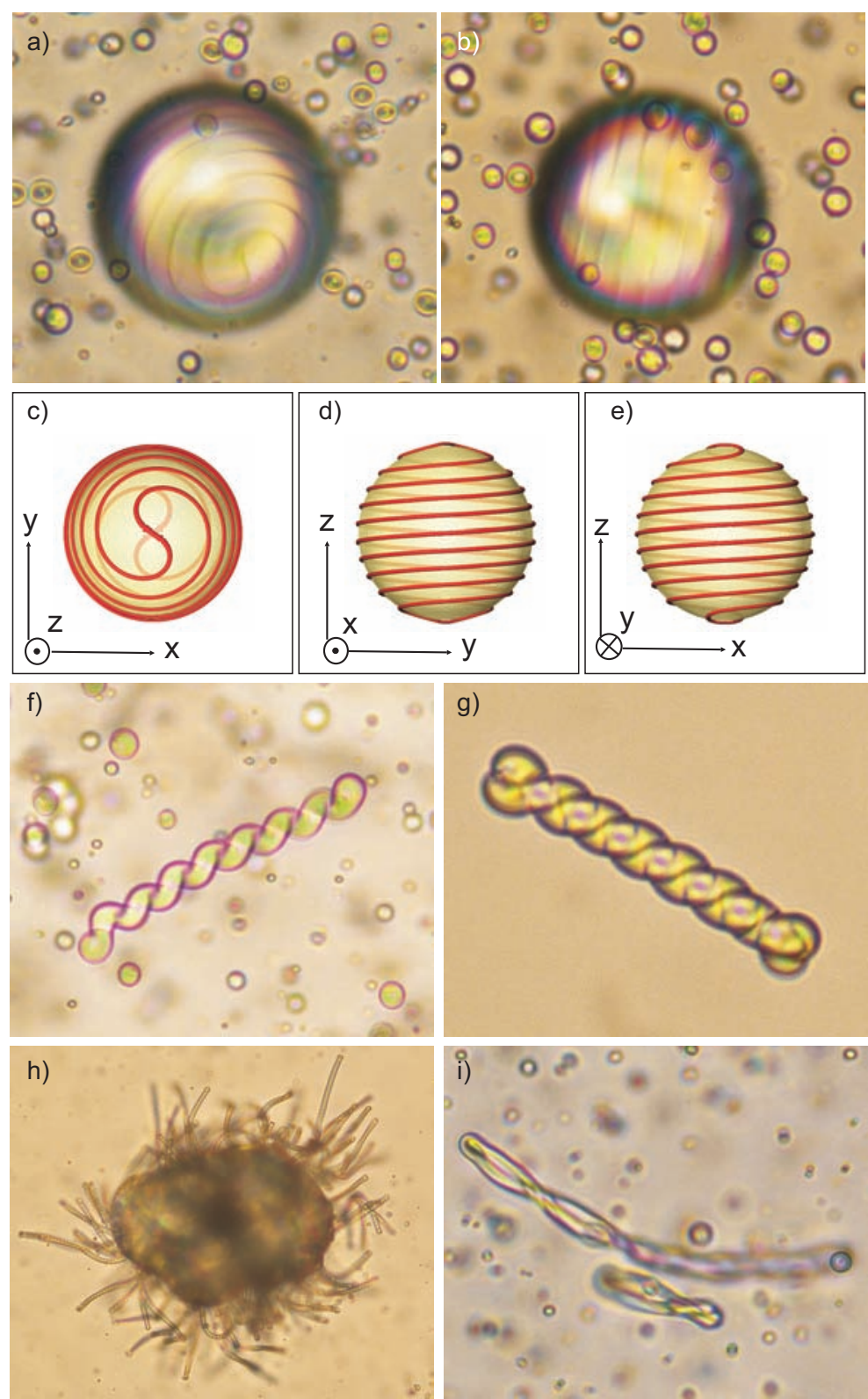

Figure 7: Cholesteric droplets in aqueous micellar solutions. Surrounding aqueous solutions in all the above cases contain $0.75 \mathrm{mM} \mathrm{CTAB}$ (a higher homologue of TTAB). (a-b) White light images of a $35 \mu \mathrm{m}$ cholesteric droplet. (c-e) Schematic representation of the defect line orientation on the surface of the above cholesteric droplet. Images are provided by Miha Ravnik (University of Ljubljana). (f-g) If the interfacial tension between LC-aqueous solution is significantly low, cholesteric droplets spontaneously expand into either singleor double-stranded helical filaments. Horizontal widths of the images 'f' and 'g' are 72 $\mu \mathrm{m}$ and $24 \mu \mathrm{m}$ respectively. (h) In the case of a very big cholesteric droplet, multiple double-stranded (or single) filaments emanate from the surface of the droplet. The resulting cholesteric droplet looks like an organism with multiple tails. Horizontal width of the image is $216 \mu \mathrm{m}$. (i) Cholesteric droplets can also morph into plectonemic supercoils with different writhes. Circular DNA molecules also form similar supercoiled structures [24]. Horizontal width of the image is $58 \mu \mathrm{m}$. 


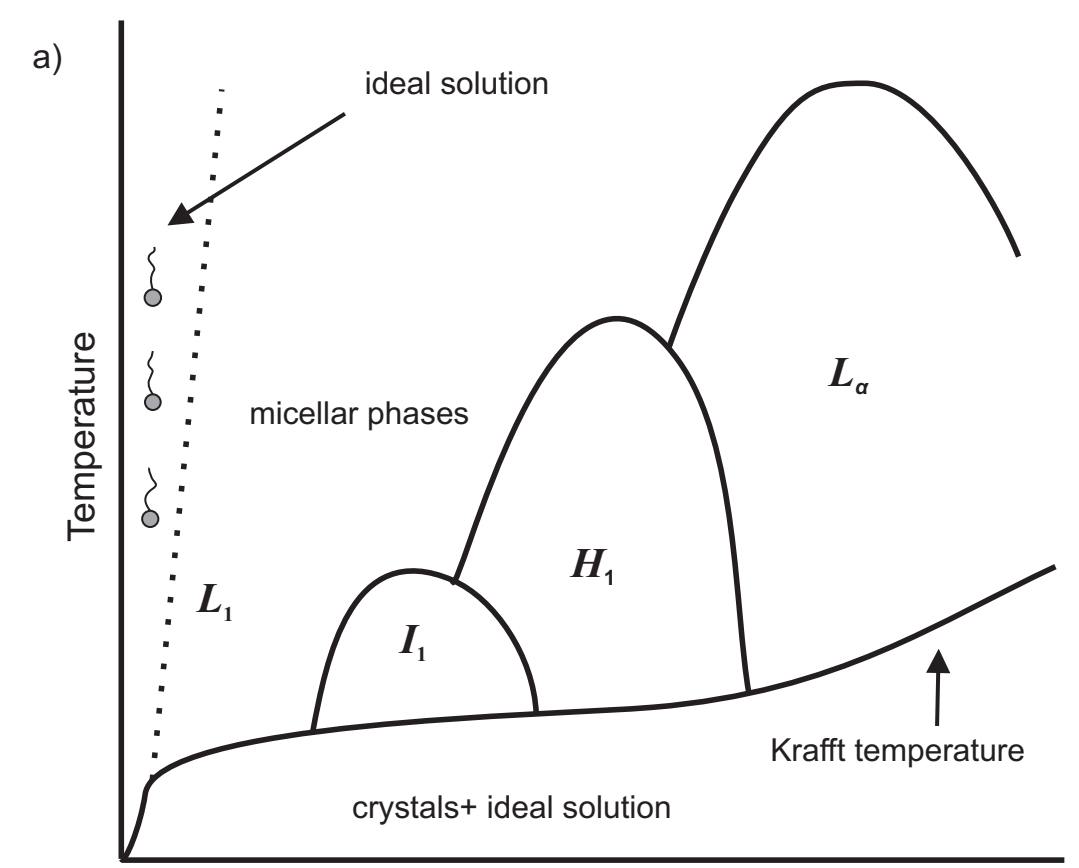

Amphiphile concentration

b)

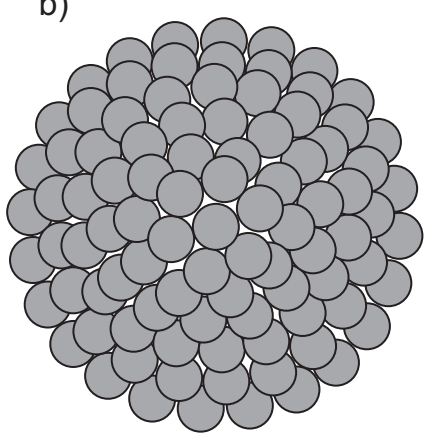

e)

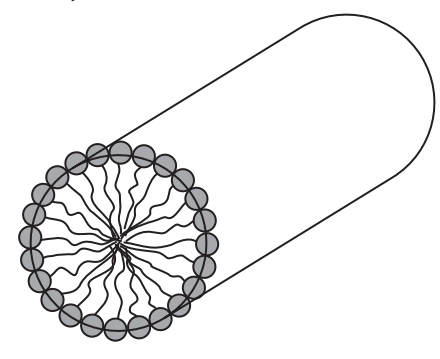

c)

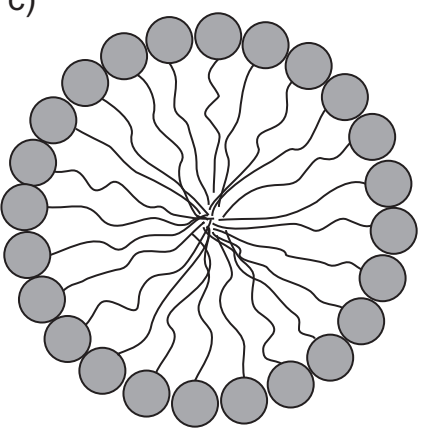

f)

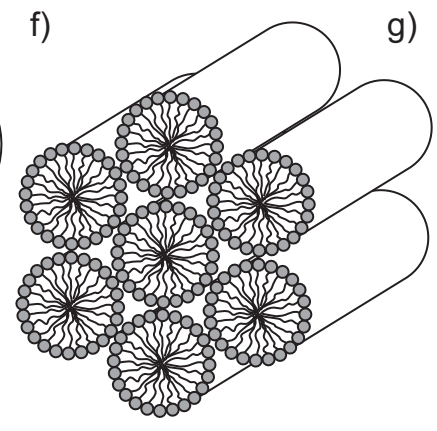

d)

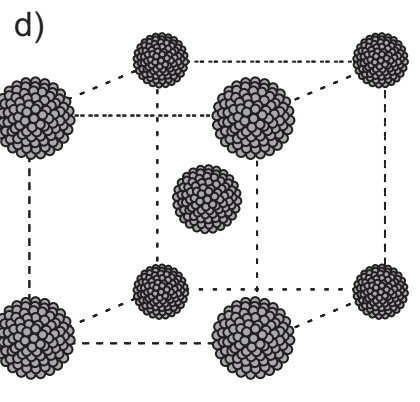

g)

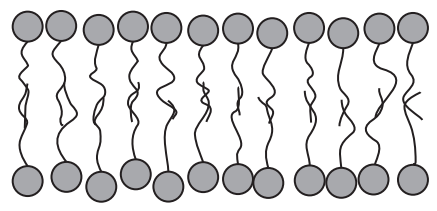
water

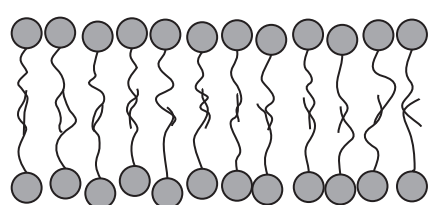

Figure 8: A typical phase diagram of an amphiphile-water system. (a) This diagram clearly shows that the formation of lyotropic LC phases depends on both the temperature and the amphiphile concentration. An ideal solution means an aqueous solution that contains amphiphiles in the monomer state. (b-g) Schematic representation of the various structures formed by amphiphiles in water. (b) Spherical micelle. (c) Cross-sectional image of the spherical micelle. (d) Cubic lyotropic LC phase. Micelles are arranged in a body-centered cubic structure. (e) Cross-sectional image of a cylindrical micelle. (f) Hexagonal lyotropic LC phase. (g) Lamellar lyotropic LC phase. 
between two phases. As an amphiphile contains the regions that have affinity towards both phases, energetically it is favorable for it to adsorb onto the surface or interface of the system $^{6}$. Due to this reason, the amphiphiles are also known as surface active agents or surfactants. This specific property of the surfactants is used in many practical applications like wetting and emulsifying. If the concentration of the surfactant in water exceeds the CMC level, insoluble organic molecules (oils) can be solubilized in the hydrophobic regions of the micelles. This oil solubilization capacity of aqueous micellar solutions is used in applications such as cleaning and drug delivery.

\subsection{Thesis outline}

In this dissertation, we focussed on the interactions between various thermotropic liquid crystals and aqueous surfactant solutions. The internal molecular arrangement of the thermotropic liquid crystals has striking influence on the behavior of LC-aqueous interfaces. Thus, we investigated several LC-aqueous systems and observed the formation of variety of $\mathrm{LC}$ objects at their respective LC-aqueous interfaces. The non-equilibrium dynamics of the LC-aqueous systems and the optical applications of some of the LC objects are reported. The LC-aqueous systems at equilibrium are also characterized by using rheological and neutron scattering techniques.

This thesis is organized in the following manner. Chapter 2 presents the materials and the experimental techniques that have been used to investigate the interfacial behavior of various thermotropic LC-aqueous systems. The insertion of an LC droplet in an aqueous micellar solution results in the complete solubilization of the droplet. Solubilization of an LC droplet is always accompanied by the self-propelled motion of the droplet. The effect of various parameters such as temperature and surfactant concentration on solubilization and the self-propelled motion of nematic LC droplets are extensively discussed in the first part of Chapter 3 (nematic LCs in aqueous ionic micellar solutions). Solubilization of the thermotropic LCs in aqueous ionic micellar solutions generally makes the aqueous solutions extremely viscous. Thus, rheological properties of the resulting aqueous solutions, as well as the micellar morphologies, are investigated using a micro-rheometer and small-angle neutron scattering technique (SANS). Ultra-low interfacial tension values between nematic LCs and aqueous micellar solutions result in the formation of nematic filaments. Structural and stability analyses of these filaments are also presented in this nematic LCs section. The second part of the Chapter 3 (smectic LCs in aqueous ionic micellar solutions) provides the structural analysis of various self-assembled smectic LC objects and subsequently demonstrates their optical applications. Finally, the main concluding remarks of the thesis are presented in Chapter 4.

\footnotetext{
${ }^{6}$ The boundaries between solid-liquid and liquid-liquid systems are called "interfaces", whereas the boundary between a liquid-gas system is termed a "surface".
} 


\section{Materials and methods}

\section{$2.1 \quad$ Materials}

All the employed thermotropic LC materials and their respective phase transition temperatures are listed in Table 1. All the LCs, except C7 and 8.0.6, were obtained from SYNTHON Chemicals and used as received without any further purification. $\mathrm{C} 7$ and 8.0.6 were synthesized in the lab of Prof. Dr. Gerd Heppke, Technische Universität Berlin.

\begin{tabular}{|c|c|c|c|}
\hline Liquid crystal & Chemical name & $\begin{array}{l}\text { Phase transition } \\
\text { temperature }\left[{ }^{\circ} \mathrm{C}\right]\end{array}$ & CAS number \\
\hline $2 \mathrm{CB}$ & 4-Ethyl-4'-cyanobiphenyl & Cr 75 [N 22] Iso & $58743-75-2$ \\
\hline $3 \mathrm{CB}$ & 4-Propyl-4'-cyanobiphenyl & Cr 67.3 (N 30.3) Iso & $58743-76-3$ \\
\hline $4 \mathrm{CB}$ & 4-Butyl-4'-cyanobiphenyl & Cr 48 (N 16.5) Iso & $52709-83-8$ \\
\hline $5 \mathrm{CB}$ & 4-Pentyl-4'-cyanobiphenyl & Cr 24 N 35.3 Iso & $40817-08-1$ \\
\hline $6 \mathrm{CB}$ & 4-Hexyl-4'-cyanobiphenyl & Cr 14.3 N 30.1 Iso & $41122-70-7$ \\
\hline $7 \mathrm{CB}$ & 4-Heptyl-4'-cyanobiphenyl & $\begin{array}{l}\text { Cr' } 15.0 \text { Cr } 30.0 \mathrm{~N} \\
42.8 \text { Iso }\end{array}$ & $41122-71-8$ \\
\hline $8 \mathrm{CB}$ & 4-Octyl-4'-cyanobiphenyl & $\begin{array}{l}\text { Cr 21.5 SmA } 33.5 \mathrm{~N} \\
40.5 \text { Iso }\end{array}$ & $52709-84-9$ \\
\hline $9 \mathrm{CB}$ & 4-Nonyl-4'-cyanobiphenyl & $\begin{array}{l}\text { Cr' 29.5 Cr } 42.0 \text { SmA } \\
48.0 \mathrm{~N} 49.5 \text { Iso }\end{array}$ & $52709-85-0$ \\
\hline $10 \mathrm{CB}$ & 4-Decyl-4'-cyanobiphenyl & Cr 44.0 SmA 54.5 Iso & $59454-35-2$ \\
\hline $11 \mathrm{CB}$ & 4-Undecyl-4'-cyanobiphenyl & Cr 53.0 SmA 57.5 Iso & $65860-74-4$ \\
\hline $12 \mathrm{CB}$ & 4-Dodecyl-4'-cyanobiphenyl & Cr 48.0 SmA 58.5 Iso & $57125-49-2$ \\
\hline MBBA & $\begin{array}{l}\text { N-(4-Methoxybenzylidene) } \\
\text {-4-butylaniline }\end{array}$ & Cr 22.0 N 48.0 Iso & $97402-82-9$ \\
\hline EBBA & $\begin{array}{l}\text { N-(4-Ethyloxybenzylidene) } \\
\text {-4-butylaniline }\end{array}$ & Cr 36.5 N 79.8 Iso & $98814-59-6$ \\
\hline $\mathrm{C} 7$ & $\begin{array}{l}\text { 4-(2S,3S)-(2-Chloro- } \\
\text {-3-methylpentanoyloxy)-4' } \\
\text {-heptyloxybiphenyl }\end{array}$ & $\begin{array}{l}\text { Cr 55.0 (SmG* } 44.0 \\
\left.\mathrm{SmC}^{*} 55.0\right) \mathrm{SmA}^{*} 62.0 \text { Iso }\end{array}$ & $100497-43-6$ \\
\hline 8OPhPy8 & $\begin{array}{l}\text { 5-Octyl-2-(4-octyloxyphenyl) } \\
\text { pyrimidine }\end{array}$ & $\begin{array}{l}\text { Cr 28.5 SmC } 55.5 \mathrm{SmA} \\
62 \mathrm{~N} 68 \text { Iso }\end{array}$ & $57202-50-3$ \\
\hline 8.0 .6 & $\begin{array}{l}\text { 4-(Hexyloxy)phenyl 4' } \\
\text {-(octyloxy)benzoate }\end{array}$ & $\begin{array}{l}\text { (39.0) Cr 55.0 SmC } 66.0 \\
\text { N 89.0 Iso }\end{array}$ & $54963-63-2$ \\
\hline
\end{tabular}

Table 1: Phase transition temperatures of LCs. Monotropic phases and extrapolated phases are denoted in round and square brackets respectively. Cr \& Cr': crystal phases; N: nematic; SmA: smectic-A; SmC: smectic-C; SmA* \& SmC*: chiral versions of the smectic-A and smectic-C phases; SmG* : chiral version of crystal $\mathrm{G}$ phase; and "Iso" represents an isotropic phase. 
Most of the work was undertaken with three room temperature LCs, namely, 5CB (4-pentyl-4'-cyanobiphenyl), MBBA (4-methoxybenzylidene-4'-butylaniline), and 8CB (4octyl-4'-cyanobiphenyl), due to convenience. At room temperature, 5CB and MBBA are in the nematic state and $8 \mathrm{CB}$ forms a smectic-A phase. Another reason for selecting these LCs is that several homologues of these compounds are also commercially available. Cholesteric nematic phases were produced by doping the above three compounds with different chiral compounds (Table 2). The chiral dopants CB15 and R811 induce right-handed helix structures whereas S811 induce left-handed helix structures. The helix pitch decreases with increasing concentration of the chiral dopant. The helix pitch also generally decreases with increasing temperature (reverse trends are also observed in some cases). In the case of the chiral smectic-C phase $(\mathrm{C} 7)$, the effect of temperature on helix pitch is generally opposite to that seen in the chiral nematic phase. If a material exhibits both cholesteric and smectic- $\mathrm{C}^{*}$ phases, then generally the helix pitch is relatively shorter in the former.

\begin{tabular}{|c|l|l|l|}
\hline Chiral dopant & Chemical name & CAS number & Supplier \\
\hline CB15 & $\begin{array}{l}\text { (S)-4-Cyano-4'-(2-methylbutyl) } \\
\text { biphenyl }\end{array}$ & $63799-11-1$ & Synthon Chemicals \\
\hline S811 & $\begin{array}{l}\text { (S)-Octan-2-yl 4-((4-(hexyloxy) } \\
\text { benzoyl)oxy)benzoate }\end{array}$ & $87321-20-8$ & Merck KGaA \\
\hline R811 & $\begin{array}{l}\text { (R)-Octan-2-yl 4-((4-(hexyloxy) } \\
\text { benzoyl)oxy)benzoate }\end{array}$ & 133676-09-2 & Merck KGaA \\
\hline
\end{tabular}

Table 2: Chiral dopants. S811 and R811 are in a solid state at room temperature whereas CB15 is in a liquid state.

All the thermotropic LCs listed in Table 1 are sparingly soluble in water. Thus, a pure LC-pure water interface is extremely inactive. In order to activate the LC-aqueous interface, small amounts of surfactants must be added to at least one of the phases. A complete list of the surfactants that were used to activate the LC-aqueous interfaces are provided in Table 3. All the surfactants, except the last two amphiphiles in Table 3, were obtained from Sigma-Aldrich Laborchemikalien GmbH (Seelze, Germany) and were used as received. If the concentration of surfactants in the aqueous phase is above their respective CMC values, the micellar solubilization of LC molecules is observed (see Section 3.1.2).

Thermotropic LCs form filament-like structures at LC-aqueous interfaces during the solubilization of LC droplets in aqueous ionic surfactant solutions. The morphology of the observed filaments at LC-aqueous interfaces depends significantly on the phase of LC molecules. The structure of smectic-A filaments closely resembles heavily reported lyotropic myelin figures. Thus, a significant part of the thesis is devoted to finding the similarities and differences between smectic-A filaments and classical lyotropic myelin figures. We studied the smectic-A filament formation, growth and stability using two experimental configurations (Section 3.2.3: sandwich and capillary methods). For sandwich experiments, glass slides and cover slips were bought from VWR International GmbH (Hannover, Germany). In the case of capillary experiments, square glass capillaries of several sizes (length $=5 \mathrm{~cm}$; side length of the square cross section: 35, 50, 80 and $100 \mu \mathrm{m}$ ) were obtained from CM Scientific Ltd., United Kingdom. Any LC filament growth and stability depends significantly on the interfacial tension between LC and the surrounding aqueous solution. The concentration of surfactants and salts significantly affects the interfacial tension values. As salt, we used sodium chloride ( $\mathrm{NaCl}$, Sigma-Aldrich, used as received). Ultimately, these LC filaments are only transient structures which make them inadequate for any practical 


\begin{tabular}{|c|c|c|c|}
\hline Amphiphile & Chemical name & $\begin{array}{l}\mathrm{CMC} \text { value in water } \\
\text { at } 25^{\circ} \mathrm{C}(\mathrm{mM})\end{array}$ & CAS number \\
\hline $\operatorname{DeTAB}\left(\mathrm{C}_{10} \mathrm{TAB}\right)$ & $\begin{array}{l}\text { Decyltrimethylammonium } \\
\text { bromide }\end{array}$ & 68 & $2082-84-0$ \\
\hline $\operatorname{DTAB}\left(\mathrm{C}_{12} \mathrm{TAB}\right)$ & $\begin{array}{l}\text { Dodecyltrimethylammonium } \\
\text { bromide }\end{array}$ & 16 & $1119-94-4$ \\
\hline TTAB $\left(\mathrm{C}_{14} \mathrm{TAB}\right)$ & $\begin{array}{l}\text { Tetradecyltrimethylammonium } \\
\text { bromide }\end{array}$ & 3.6 & $1119-97-7$ \\
\hline CTAB $\left(\mathrm{C}_{16} \mathrm{TAB}\right)$ & $\begin{array}{l}\text { Hexadecyltrimethylammonium } \\
\text { bromide }\end{array}$ & 0.98 & 57-09-0 \\
\hline SDS & Sodium dodecyl sulfate & 8.2 & $151-21-3$ \\
\hline $\mathrm{C}_{12} \mathrm{E}_{3}$ & $\begin{array}{l}\text { Triethylene glycol monododecyl } \\
\text { ether }\end{array}$ & 0.052 & $3055-94-5$ \\
\hline Monoolein & 1-Oleoyl-rac-glycerol & $\begin{array}{c}10.5 \mathrm{mM} \text { in } \\
\text { benzene at } 30^{\circ} \mathrm{C}\end{array}$ & $111-03-5$ \\
\hline $\mathrm{PC}$ & $\begin{array}{l}\text { L- } \alpha \text {-phosphatidylcholine } \\
\text { (Egg, Chicken) }\end{array}$ & $<10^{-9}$ & $97281-44-2$ \\
\hline PS & $\begin{array}{l}\text { L- } \alpha \text {-phosphatidylserine } \\
\text { (Brain, Porcine) (sodium salt) }\end{array}$ & Between $10^{-9}$ and $10^{-5}$ & $383907-32-2$ \\
\hline
\end{tabular}

Table 3: Critical micellar concentration values of amphiphiles. An extensive list of the CMC values of various amphiphiles is available in the reference publication [14]. The CMC value of monoolein in water is not available.

application. One possible way to stabilize them is to freeze their structures by using a UV polymerization technique (Section 3.2.5). Liquid crystal was doped with a monomer (RM 305), a cross linker (RM 257) and a photo-initiator (Irgacure 907). Filaments formed by this mixture in an aqueous solution were immobilized by using $320 \mathrm{~nm}$ UV light. RM 305 and RM 257 were obtained from Merck, UK. Irgacure 907 was freely provided by the Dispersions \& Pigments division, BASF (Germany).

The properties of the lyotropic myelin figures were investigated by using three different amphiphiles (Triethylene glycol monododecyl ether $\left(\mathrm{C}_{12} \mathrm{E}_{3}\right)$, L- $\alpha$-phosphatidylcholine (PC) and L- $\alpha$-phosphatidylserine (PS)). Phospholipids (PC and PS) were obtained from Avanti Polar Lipids (Alabaster, AL, USA), whereas $\mathrm{C}_{12} \mathrm{E}_{3}$ was purchased from Sigma-Aldrich.

\subsection{Experimental methods}

Several experimental techniques were employed to investigate the LC-aqueous systems. In this section, we briefly discuss the specifications of all the instruments that were used to explore the LC-aqueous systems.

\subsubsection{Characterization of the micellar solutions}

The micellar solubilization of LC molecules in aqueous solutions generally produces very viscous clear solutions, provided there are enough micelles in the aqueous solutions to accommodate all the LC molecules. The rheological properties of these viscous solutions are investigated using an Anton Paar MCR 501 rheometer. Both steady state shear flow and oscillatory tests were carried out at $23^{\circ} \mathrm{C}$ using a cone-plate geometry (a cone of $25 \mathrm{~mm}$ 
diameter and cone angle of $1^{\circ}$ ). This geometry consists of a rotating upper cone and a fixed bottom plate, with a measurement gap of $50 \mu \mathrm{m}$. A solvent trap was used to minimize the solvent (water) evaporation problem. Stress sweeps at a frequency of $1 \mathrm{~Hz}$ were performed to estimate the linear viscoelastic range. The alteration of the viscosity of an aqueous solution before and after the addition of solubilizates is due to the change in structural and electrical properties of the micelles. Preliminary small-angle neutron scattering (SANS) experiments were performed by my colleague (Dr. Lucas Goehring) for obtaining structural properties of the micelles. The aqueous solutions were placed in $0.2 \mathrm{~mm}$ thick quartz-glass Hele-Shaw cells, and capped with tape to prevent drying. In these experiments, the detector positions used were $1.2 \mathrm{~m}$ and $4 \mathrm{~m}$ with a wavelength of $6 \AA$. This gives a scattering vector $(\mathrm{Q})$ range between 0.008 and $0.17 \AA^{-1}$. The raw scattering spectra were corrected for detector efficiency, absorption, solvent $\left(\mathrm{D}_{2} \mathrm{O}\right)$ scattering and instrumental background. The experiments were carried out at $25^{\circ} \mathrm{C}$.

\subsubsection{Polarizing optical microscopy}

The anisotropic shapes of LC molecules give rise to anisotropy in refractive indices. We restrict our discussion to uniaxial materials as all the LCs used in this thesis are uniaxial in nature. As shown in Fig. 9(a), uniaxial LC molecules have only two principle refractive indices $-\mathrm{n}_{\|}$and $\mathrm{n}_{\perp}$. When the unpolarized light enters into an uniaxial LC material, it may decompose into fast (ordinary ray) and slow (extraordinary ray) components. The refractive indices of the LC medium that the ordinary and extraordinary rays experience are denoted by $\mathrm{n}_{o}$ (ordinary refractive index) and $\mathrm{n}_{e}$ (extraordinary refractive index). The $\mathrm{n}_{o}$ value is always constant and equal to $\mathrm{n}_{\perp}$. But the $\mathrm{n}_{e}$ value depends on the direction of the light propagation, i.e., it depends on $\phi$, the angle between the optic axis and the direction of light propagation. The relation between extraordinary refractive index $\mathrm{n}_{e}$ and $\phi$ is provided by

$$
n_{e}=\frac{n_{\|} n_{\perp}}{\sqrt{n_{\|}^{2} \cos ^{2} \phi+n_{\perp}^{2} \sin ^{2} \phi}},
$$

and the birefringence of the LC medium is characterized by

$$
\delta n=n_{e}-n_{o}
$$

Because the electric field component of the incident light experiences different refractive indices, the waves become out of phase as they propagate in the LC medium. Thus, the polarization state of the light is not the same before and after passing through the LC medium. Any polarization state can be obtained from the incident unpolarized light by choosing the right combination of birefringent material and sample thickness. All the LCs listed in Table 1 are known as positive uniaxial LCs because the condition $\delta n>0$ holds true for all of them.

Polarizing optical microscopy (POM) helps us to use the birefringent nature of the LCs for the visualization of the macroscopic molecular orientation. Fig. 9(b) presents the simplified POM experimental technique in the transmission mode. An unpolarized white light source and an ocular lens (or a video camera) are present at the extreme ends of the setup. LC samples are always placed on a rotating stage that exists between two linear polarizers. The relative positions of the polarizer axes of the two polarizers can be easily adjusted to the minimum precision of $1^{\circ}$. The most often used crossed polarized state consists of the polarizer axes in a mutually perpendicular position. In this crossed polarized 


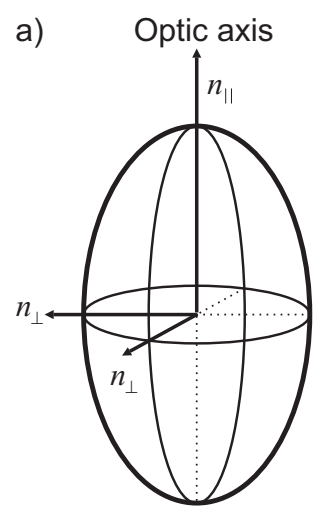

c)

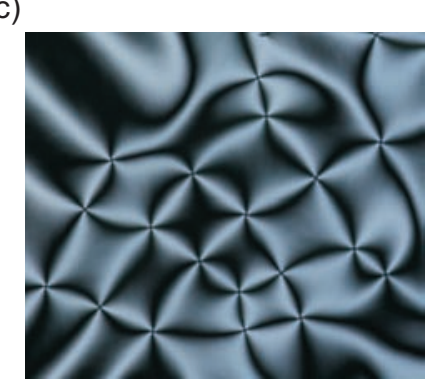

b)

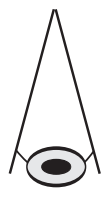

Eyepiece
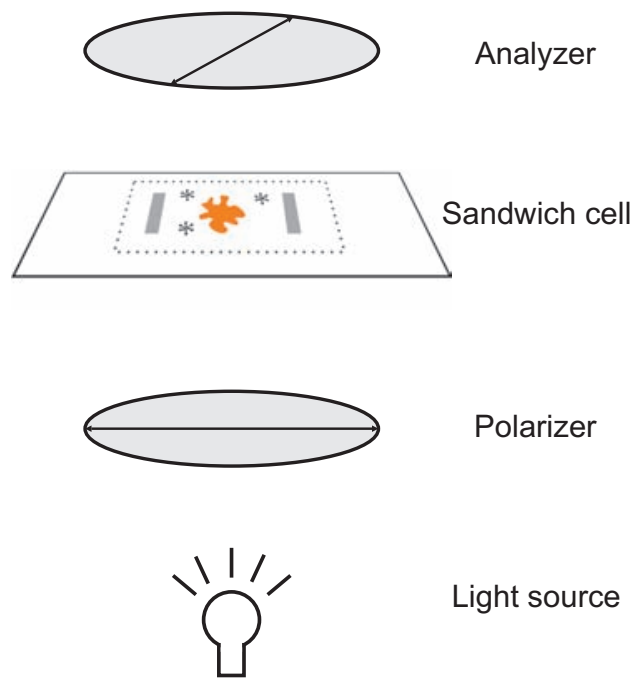

Polarizer

Light source

Figure 9: Schematic view of the polarizing optical microscopy technique and an image of Schlieren textures. (a) Schematic representation of the two principal refractive indices of a uniaxial LC material. (b) Schematic diagram of a simplified configuration of a polarizing optical microscope. Sandwich cell: An LC droplet (orange color region) is sandwiched between two glass plates which are separated by thin spacers. The LC droplet is surrounded by an aqueous micellar solution. (c) A thin 5CB (nematic phase) film under crossed polarizers. Horizontal width of the image is $255 \mu \mathrm{m}$.

state, no light will pass through the second polarizer (also known as the analyzer) in the absence of any material with a non-zero birefringence. As shown in Fig. 9(c), a randomly oriented nematic LC produces a beautiful pattern (Schlieren texture) when viewed under crossed polarizers. Any dark region in the schlieren texture represents the local director that is aligned parallel or perpendicular to the polarizer or analyzer axes.

Our lab is equipped with a Nikon Eclipse LV 100 (Japan) polarizing optical microscope and a Nikon DS-Fi1 digital camera. Temperature controlled POM experiments were conducted by using a Linkam THMSG600 micro heating stage (connected to a TMS 94 temperature controller) mounted in the microscope between the polarizer and analyzer. A retardation plate $(\lambda=530 \mathrm{~nm})$ was used very often to determine the internal molecular arrangement of the weakly birefringent or thin filaments. But, simple POM observations are not enough to obtain full 3D structures of the filaments. Thus, the fluorescence confocal polarizing microscopy (FCPM) technique is used to determine the 3D structure of smectic-A filaments (Section 3.2.2).

\subsubsection{Fluorescence confocal polarizing microscopy}

The fluorescence confocal polarizing microscopy technique was developed by Smalyukh, Shiyanovskii and Lavrentovich $[29,30]$, and the principle scheme of the FCPM technique is provided in Fig. 10(a). This technique is just an extension of widely used confocal fluorescence microscopy (CFM) with just two modifications [31]. First, an LC sample is doped with a strongly anisometric fluorescent dye, which preferentially aligns along the director 
of the LC sample. Due to this preferential alignment of the dye in the LC host, excitation/fluorescence transition dipoles of the dye follow the local director. Second, the dyedoped LC sample is probed with a linearly polarized laser light source. Because of these two modifications, the maximum (minimum) intensity of the fluorescence is observed when the polarization of the laser beam is parallel (perpendicular) to the LC director. This capability to image three-dimensional patterns of orientational order gives the FCPM technique an important advantage over the CFM technique.

In order to verify whether our selection of dye (Nile red, Sigma-Aldrich) is suitable for the FCPM experiments, a simple test was performed. An 8CB (doped with $0.01 \mathrm{wt} \%$ Nile red) smectic sandwich cell was prepared. The director of $8 \mathrm{CB}$ in the cell was aligned uniformly in the plane of the cell. This cell was exposed to a linearly polarized laser light source and the emitted fluorescent light was collected by using an imaging spectrometer (Andor, Shamrock SR-500i). As shown in Fig. 10(b), the emitted fluorescent intensity was at its maximum when the polarization of incident laser light was parallel to the director. This strong orientation of Nile red molecules along the local director of the $8 \mathrm{CB}$ smectic-A sample allowed us to obtain the 3D structure of $8 \mathrm{CB}$ smectic-A filaments.

When an $8 \mathrm{CB}$ filament grows at the $8 \mathrm{CB}$ bulk (doped with 0.01 wt\% Nile red)-aqueous interface, Nile red molecules also diffuse into the filament along with $8 \mathrm{CB}$ molecules from the bulk. Based on the above experimental evidence, we presume that dye molecules are aligned along $8 \mathrm{CB}$ molecules in the filament. Our lab is equipped with a Leica TCS-SP2 confocal microscope. It contains a linearly polarized He-Ne laser $(\lambda=543 \mathrm{~nm})$ which is used to excite the dye molecules in the filament. One needs to be very careful regarding the dye concentration and the intensity of the laser, as the filament is extremely sensitive to changes in temperature. If the exposed region of the filament is overheated, $8 \mathrm{CB}$ starts to leak into the surrounding aqueous solution in the form of tiny filament-like structures (Section 3.2.4).

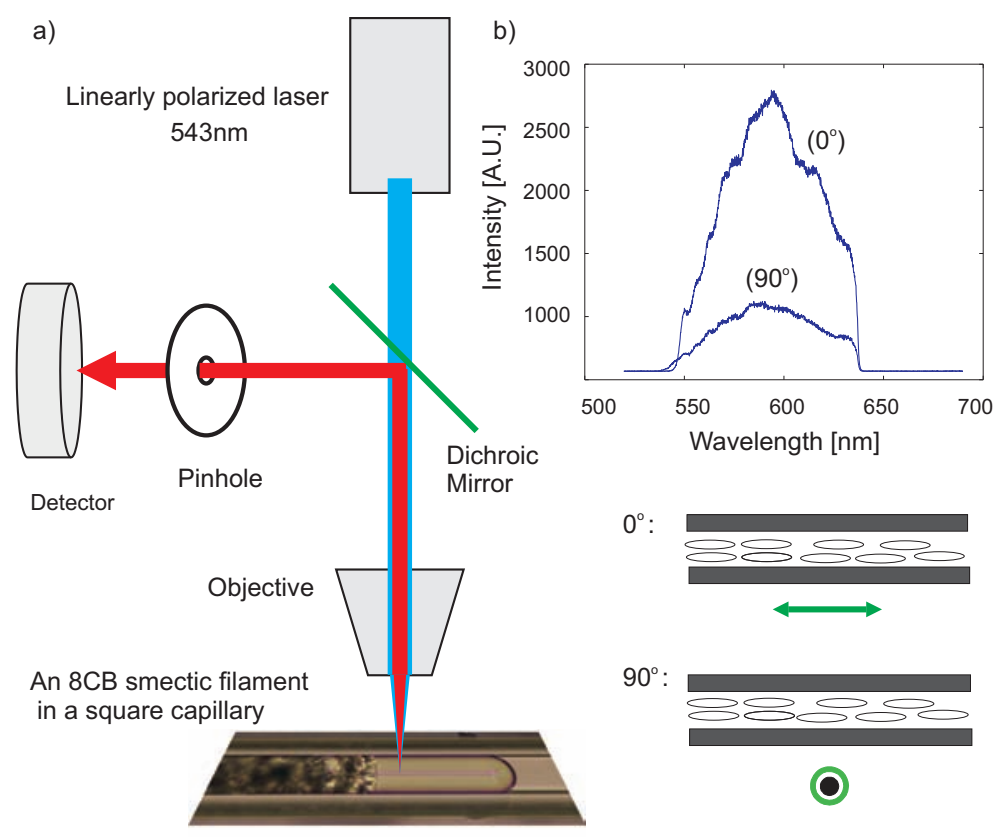

Figure 10: Schematic view of the fluorescence confocal polarization microscopy and polarization dependant intensity measurements. (a) Schematic illustration of the FCPM technique. (b) Maximum fluorescent light is emitted by Nile red when the polarization of the incident laser beam is parallel $\left(0^{\circ}\right)$ to the $8 \mathrm{CB}$ director. The cell is made of unidirectionally rubbed polyimide substrates. 
A photomultiplier tube (PMT) is used to detect a selected region $(620-660 \mathrm{~nm})$ of the emitted fluorescent light (the excitation maximum for Nile red is at $640 \mathrm{~nm}$ ). A pinhole is placed in front of the PMT in order to allow the light that is emitted exclusively from the focussed region. The laser beam can scan the filament both horizontally and vertically. The polarization direction of the incident laser beam is always kept constant. The polarization dependence of the fluorescence emission from the $8 \mathrm{CB}$ smectic-A filament is obtained by rotating the filament in a plane horizontal to the microscope stage. The results obtained from this technique are presented in Section 3.2.2.

\subsubsection{Optical setup for waveguiding experiments}

All the work related to the optical applications of smectic filaments (Section 3.2.7) was carried out in the soft matter laboratory, Jožef Stefan Institute, Slovenia. All the optical experiments were performed on an inverted optical microscope (Nikon Eclipse TE2000-U) which was placed on a vibration-free optical table. The schematic representation of the whole optical setup is presented in Fig. 11 and the real view of the setup is provided in Fig. 12. The sandwich cells that contain freely floating smectic filaments were mounted on a motorized $\mathrm{X}-\mathrm{Y}-\mathrm{Z}$ stage (Prior OptiScan II). This stage was controlled by a joystick (or a computer program) and this option greatly helped to move the stage (cell) very precisely. For realizing the waveguiding in smectic filaments, two lasers were connected to the microscope from its back side. The beams generated by each one of the two laser sources were passed through acousto-optic-deflector (AOD) units, which enabled us to spatially control the optical beams. After passing through the AOD units, the beams also passed through dichroic mirror turrets, which selectively allowed certain wavelengths of the light to reflect into the back aperture of an objective. Each laser source was allotted with separate units of AOD and dichroic mirror turrets which enabled us to use them simultaneously. One of the laser sources was an infrared (IR) continuous wave (CW) fiber laser (Tweez 200si, Laser unit, Aresis) with an emission peak at $1064 \mathrm{~nm}$ and with a maximum power output of $1.7 \mathrm{~W}$. These infrared laser tweezers were used to position the smectic filaments in the aqueous solution. The second laser source was an Argon ion $\left(\mathrm{Ar}^{+}\right) \mathrm{CW}$ laser (Coherent, Innova 90C-4) with an emission peak at $514.5 \mathrm{~nm}$ and with a maximum power output of $1.5 \mathrm{~W}$. These $\mathrm{Ar}^{+}$laser tweezers were used for fluorescent excitation. Nile red and Rhodamine B (Sigma-Aldrich) were used for doping liquid crystals whose absorption peaks exist at $553 \mathrm{~nm}$ and $554 \mathrm{~nm}$ respectively. The waveguiding properties of the dye doped smectic-filaments were studied by generating fluorescent light at one end of a filament using the $\mathrm{CW} \mathrm{Ar}^{+}$laser tweezers (see Section 3.2.7.1 for further details). IR and $\mathrm{Ar}^{+}$laser tweezers were controlled by Tweez v2.1 and Tweez v2.13m software respectively. High numerical aperture long working distance objectives (Nikon NIR Apo 60X/1.0w, Nikon Plan Fluor 60X/0.7) were used for the above two laser tweezers operations.

The microscope also has one front port and two side ports. The front port was generally used for taking color pictures with a Nikon Coolpix E5400 digital camera. One of the side ports is occupied by a CMOS black and white camera (PixeLink, PLA741, $1280 \times$ 960 pixels, and physical pixel size $-6.7 \mu \mathrm{m})$. The other side port is occupied by a high resolution imaging spectrometer (Andor, Shamrock SR-500i) with a maximum resolution of $0.05 \mathrm{~nm}$. This spectrometer was used to measure the emitted fluorescence spectra of the filaments. The spectrometer has an adjustable rectangular input slit with a fixed length of $3000 \mu \mathrm{m}$ and an adjustable width in the range of $10-2500 \mu \mathrm{m}$. This input slit is positioned in the focal plane of the microscope, which enables the focused region in the sample cell to be imaged onto the input slit. Three different interchangeable diffraction gratings (300 


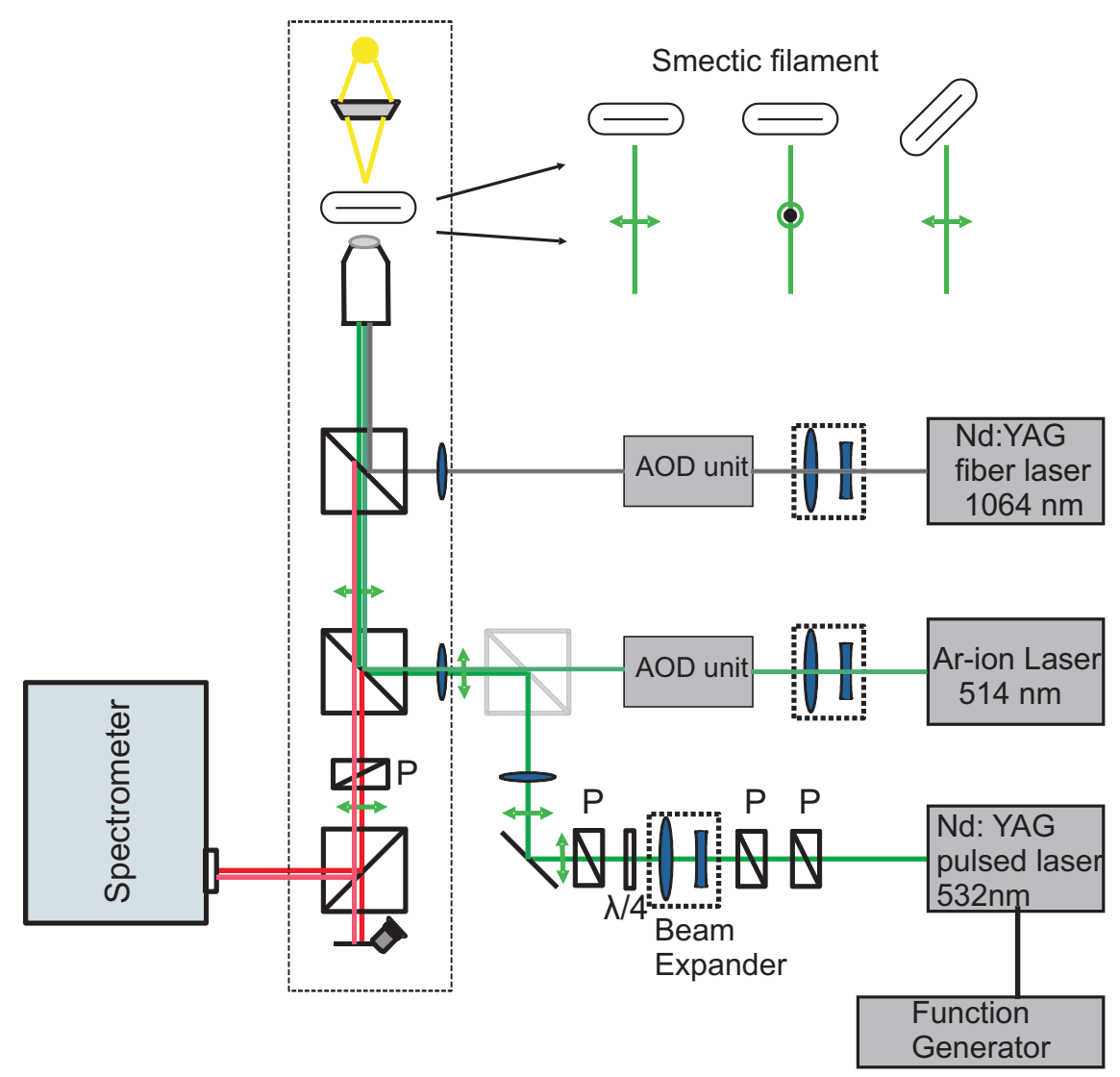

Figure 11: Schematic diagram of the optical setup for waveguiding and lasing experiments. In the case of the lasing experiments, the polarization of the incident laser beam is modified by rotating the polarizer that follows the quarter wave plate.

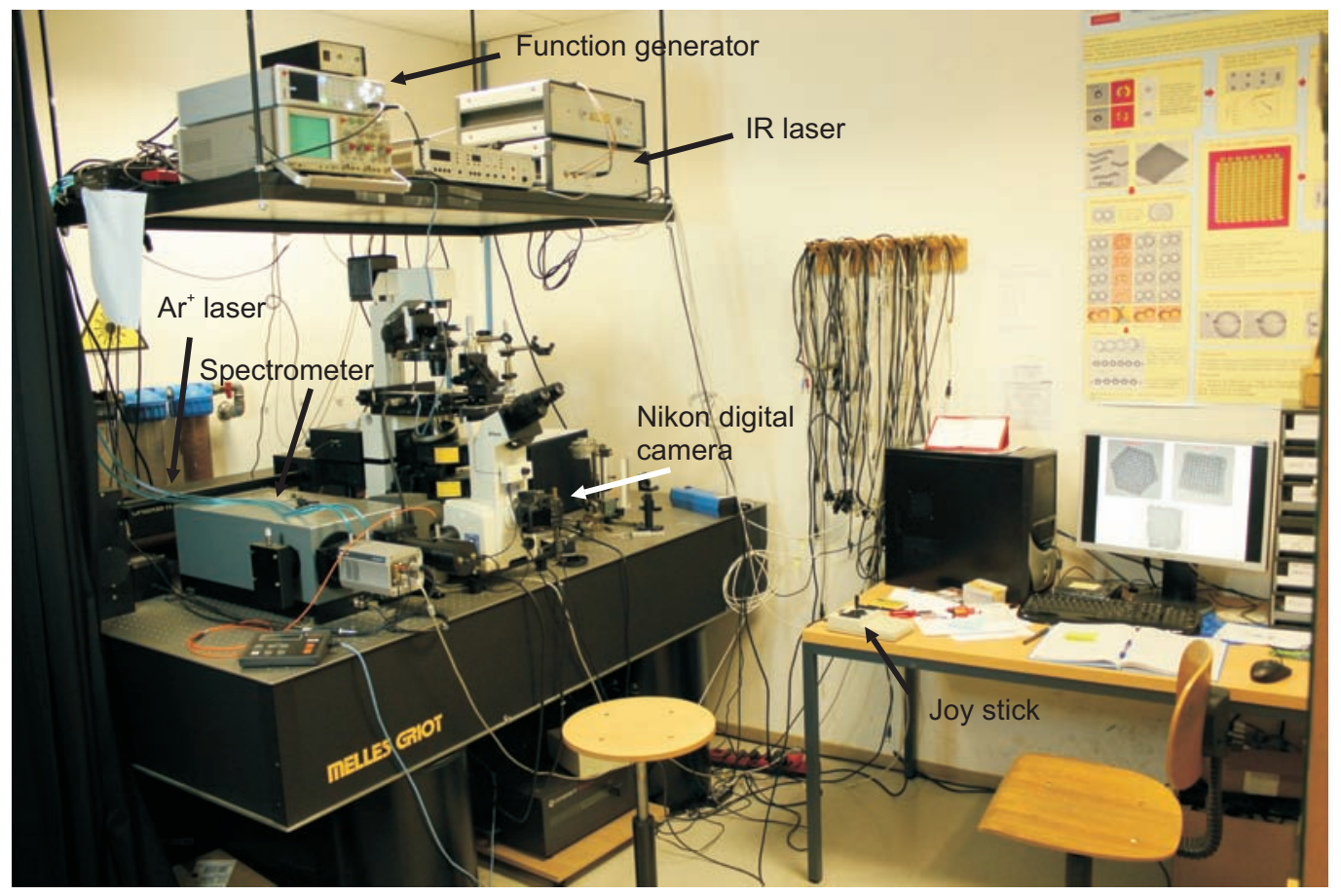

Figure 12: Photo of the actual optical setup. The real experimental setup of the laser tweezers in the soft matter laboratory, IJS, Slovenia 
lines/mm, 1200 lines/mm, 2400 lines/mm) are available for choosing an appropriate spectral range. The maximum resolution of $0.05 \mathrm{~nm}$ can be achieved by using 2400 lines/mm grating. Software was developed by Dr. Matjaž Humar for reading and analyzing spectra. An ITC503 temperature controller (Oxford Instruments) was available for heating purposes.

The photonic bandgap (PBG) characteristics of smectic- $\mathrm{C}^{*}$ filaments were investigated by using a supercontinuum laser (Fianium, SC-450). The range of the emission spectrum is from $450 \mathrm{~nm}$ to $2000 \mathrm{~nm}$. The supercontinuum laser light was produced by sending a 1064 $\mathrm{nm}$ pulsed laser beam with a repetition rate of $20 \mathrm{MHz}$ into a photonic crystal fiber.

\subsubsection{Optical setup for lasing experiments}

The lasing properties of the smectic-A filaments were investigated by using a pulsed laser (Nd:YAG $532 \mathrm{~nm}$, Alphalas, Pulselas-A-1064-500) with a $100 \mathrm{~Hz}$ repetition rate, a pulse length of $1 \mathrm{~ns}$ and a maximum pulse energy of $10 \mu \mathrm{J} .10 \mathrm{kHz}$ is the maximum possible repetition rate. This laser is attached to the microscope through an additional port that is available between the AOD unit and the dichroic mirror turret of the $\mathrm{Ar}^{+}$laser setup. Due to this arrangement, only one of these two lasers can be operated at a time. For our experiment, it was important to know the polarization of the laser light that excites the filaments. Thus, a set of optical elements was installed in between the laser source and the dichroic mirror turret. Initially, the pulsed beam was passed through two GlanTaylor polarizers. The first polarizer ensured that the laser beam was linearly polarized. The second polarizer was used to adjust the transmitted intensity of the laser beam. An adjustable Galilean beam expander was placed $\left(f_{1}=-50 \mathrm{~mm}\right.$ and $\left.f_{2}=100 \mathrm{~mm}\right)$ after these two polarizers. It was possible to produce a collimated laser beam with a beam waist as large as $100 \mu \mathrm{m}$ by using the combination of this beam expander and a $20 \mathrm{X}$ objective. A set of a quarter wave plate and a linear polarizer was placed after the beam expander just to make sure that the collimated laser beam was linearly polarized. This linear polarizer was also useful to change the polarization direction of the laser beam. The lasing thresholds were obtained by taking lasing spectra at various transmitted intensities of the pulsed laser beam. 


\section{Results and discussion}

\subsection{Nematic liquid crystals in aqueous ionic micel- lar solutions}

The dissolution behavior of isotropic oils such as alkanes, short aromatic compounds, was extensively investigated and plausible mechanisms were proposed [32-40]. Very little literature is available about how the presence of an additional orientational order in the oils (nematic liquid crystals) affect the dissolution behavior [41,42]. This chapter provides the details of solubilization rates of the different nematic liquid crystals (NLCs) in ionic micellar surfactant solutions. Based on our microscopy observations, possible solubilization pathways are discussed. Nematic liquid crystal droplets dissolve completely in the micellar solutions, provided enough active micelles are available. Rheological and structural analysis details of the resulting aqueous solutions at equilibrium are also provided. Solubilization of both the isotropic and nematic phase oil droplets result in self-propelled motion of the droplets, as well as convective flows around the droplets. Common features and differences of the solubilization of nematic liquid crystals are compared with previous micellar solubilization studies of isotropic oils. Finally, shape instabilities of the nematic liquid crystalline droplets in the aqueous medium at ultra-low interfacial tension values are discussed.

\subsubsection{Phase diagrams: Equilibrium states of NLCs in cationic micellar solutions}

A micelle in water has a hydrophobic core. This core can host (solubilize) external hydrophobic molecules (oils). The extent of the solubilization of oils in a micellar solution depends upon several properties of the micelles (aggregation number, polarity and size of individual surfactant molecules) and oil molecules (size and polarity). Investigating the equilibrium states is an essential first step in understanding the dynamics and molecular pathways of the solubilization of oils. Up to $40 \mathrm{wt} \%$, TTAB produces a micellar phase $\left(L_{1}\right)$ in water [43]. Micellar properties change with TTAB concentration. TTAB micelles are prolate ellipsoidal in shape and their half axes are $a=b=20.2 \AA$ and $c=28.9 \AA$ at $25 \mathrm{mM}$ concentration. While the minor axes $(a$ and $b)$ are found to be almost independent of the surfactant concentration, the major axis, $c$, increases from $28.9 \AA$ at $25 \mathrm{mM}(0.83 \mathrm{wt} \%)$ to $57.2 \AA$ at $673 \mathrm{mM}(18.46 \mathrm{wt} \%)$. For the same concentrations, the aggregation number of TTAB micelles increases from 90 to 203 [44].

We investigated the phase behavior of three nematic liquid crystals $(5 \mathrm{CB}, \mathrm{MBBA}$ and EBBA) in TTAB solutions (Fig. 13). Phase diagrams were made with NLC concentration as the only variable. The TTAB to water weight ratio was kept fixed at $25 \mathrm{wt} \%$. At low concentrations, all three NLCs are completely soluble as shown in Fig. 14. The saturation concentration for each NLC varies. The saturation concentration depends on the molecular 

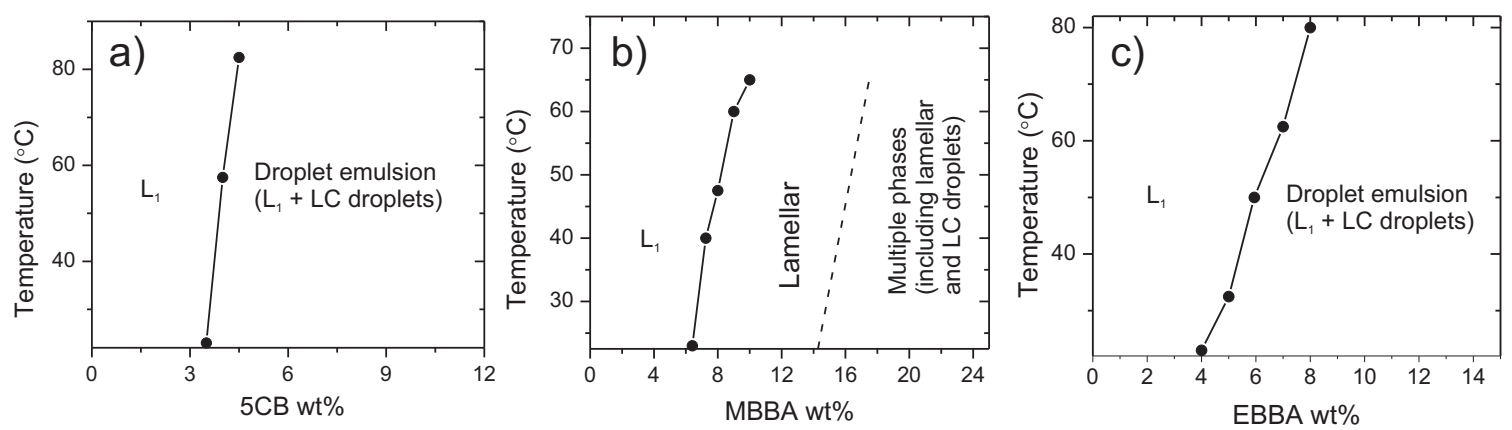

Figure 13: Phase diagrams of NLCs in TTAB 25 wt\% aqueous solutions. Phase diagrams for these ternary systems are plotted as a function of temperature and liquid crystal concentration. (a) 5CB cannot form lyotropic liquid crystalline phases in TTAB 25 wt\% solution. However, it can form a lamellar phase with more concentrated TTAB solutions such as 35 wt\%. It should be noted that pure TTAB $35 \mathrm{wt} \%$ solution is still in the $L_{1}$ phase. (b-c) Saturation solubility of EBBA is much less than MBBA, and EBBA cannot form a lyotropic liquid crystalline phase like MBBA can in TTAB $25 \mathrm{wt} \%$ solution. An increase in the chain length of a solubilizate generally reduces the solubilization limit. For concentrations between $10 \mathrm{wt} \%$ and $14 \mathrm{wt} \%$ MBBA in TTAB $25 \mathrm{wt} \%$ solutions, an unidentified multiple emulsion phase exists at high temperatures $\left(\sim>45^{\circ} \mathrm{C}\right)$.

chain length and polarity of the NLCs. The saturation concentrations of $5 \mathrm{CB}, \mathrm{MBBA}$ and EBBA are 3.5, 14 and 4 wt $\%$ respectively at $23^{\circ} \mathrm{C}$. As we increase the $5 \mathrm{CB}$ concentration beyond $3.5 \mathrm{wt} \%$, excess $5 \mathrm{CB}$ turns into nematic droplets at $23^{\circ} \mathrm{C}$ (multiple emulsion, $L_{1}+5 \mathrm{CB}$ droplets). Optically the solution turns into a turbid solution (due to the presence of nematic droplets) from a clear solution at lower $5 \mathrm{CB}$ concentrations. The average size and number density of the nematic droplets increases with increasing $5 \mathrm{CB}$ concentration. The saturation concentration increases with increasing temperature of the system. This means a turbid solution at room temperature may turn into an optically clear micellar solution as we increase the temperature. This transition between a turbid solution and a clear solution is a reversible process. There exist higher-end $5 \mathrm{CB}$ concentrations where an inverted emulsion (Fig. 15(a)) and an inverted micellar phase $\left(L_{2}\right)$ form. The $L_{2}$ phase consists of inverted micelles in the $5 \mathrm{CB}$ continuous medium. Inverted emulsions consist of aqueous droplets suspended in the $L_{2}$ phase as a continuous medium. Exact ranges of inverted emulsion and the $L_{2}$ phases are not investigated.

The MBBA phase diagram is different in comparison to $5 \mathrm{CB}$. The saturation concentration of MBBA in TTAB $25 \mathrm{wt} \%$ solution is almost four times larger than in the case of $5 \mathrm{CB}$ at $23^{\circ} \mathrm{C}$. The lengths of individual molecules of 5CB and MBBA are $18 \AA$ and $20 \AA$ respectively but $5 \mathrm{CB}$ forms dimers in the bulk, whose length reaches $25.7 \AA$ [45]. Even though both compounds are sparingly soluble in pure water, the molecular solubility of MBBA in water $(5.6 \mathrm{mg} / \mathrm{L})$ is 80 times higher than $5 \mathrm{CB}(0.07 \mathrm{mg} / \mathrm{L})$ [46]. As shown in Fig. 13 and Fig. 14, there exists a lamellar phase $\left(L_{\alpha}\right)$ in between the micellar and the multiple emulsion phases. The typical oily streak texture of a lamellar phase is observed under a polarizing microscope (Fig. 15). This lamellar phase does not seem to be a continuous single phase. Instead it appears like a mixture of $L_{1}$ and $L_{\alpha}$ and the percentage of $L_{\alpha}$ increases as the MBBA concentration increases. Solutions from this lamellar region do not phase separate even after two years. Thus, the solutions must be either single phase solutions or two phase mixtures where the two phases have almost identical densities. Detailed structural analysis is required to confirm this. It is clear that the MBBA solubilization in a TTAB micellar solution causes stronger structural changes to the TTAB micelles in comparison to $5 \mathrm{CB}$ 

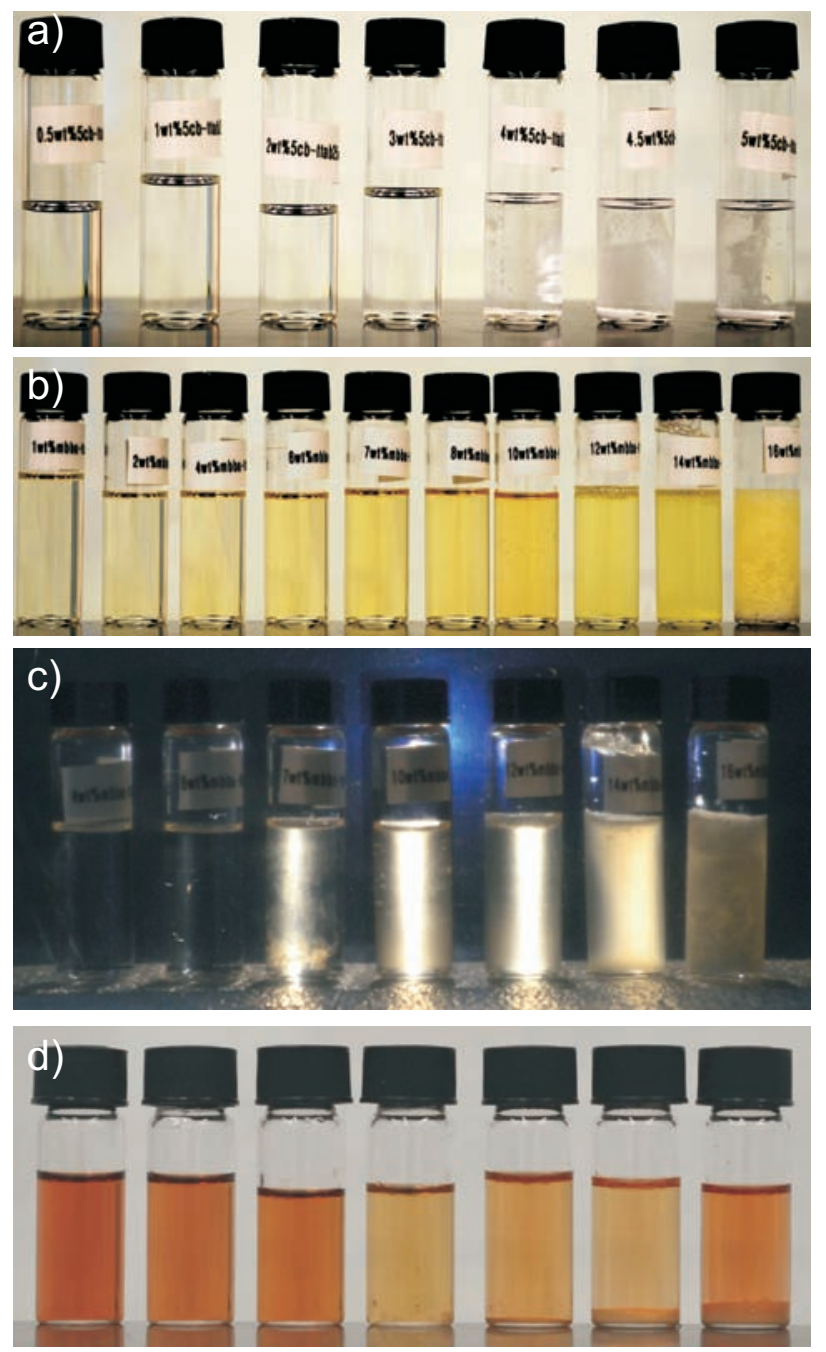

Figure 14: Visual appearances of equilibrium states of liquid crystals in TTAB 25 wt\% solutions at $23^{\circ} \mathrm{C}$. (a) Different concentrations of $5 \mathrm{CB}$ in TTAB $25 \mathrm{wt} \%$ solutions are shown (Left to right: $0.5,1,2,3,4,4.5,5 \mathrm{wt} \% 5 \mathrm{CB}$ concentrations). For concentrations above 3.5 wt\%, white color sediments exist at the bottom of the bottles. They are $5 \mathrm{CB}$ droplets that have settled due to their higher density. Predictably sediment volume fraction increases with increasing 5CB concentration (b) Equilibrium states of MBBA in TTAB $25 \mathrm{wt} \%$ solutions are shown (Left to right: $1,2,4,6,7,8,10,12,14,16 \mathrm{wt} \%$ MBBA concentrations). It is clearly evident that multiple emulsion phases exist above $14 \mathrm{wt} \%$ MBBA concentration. (c) MBBA in TTAB 25 wt\% solutions between crossed polarizers (Left to right: 4, 6, 7, 10, 12 , $14,16 \mathrm{wt} \% 5 \mathrm{CB}$ concentrations). Transition between the $L_{1}$ phase and the lamellar phase exists between 6 and 7 wt\% MBBA concentrations. (d) EBBA in TTAB 25 wt\% solutions with increasing EBBA concentrations from left to right $(1,2,3,4,5,8,11 \mathrm{wt} \%)$. As in the case of $5 \mathrm{CB}$, sediment volume fraction increases with increasing EBBA concentration for concentrations above $4 \mathrm{wt} \%$.

solubilization. Our neutron scattering data shows that the solubilization of $5 \mathrm{CB}$ in TTAB solutions does not cause significant structural changes (see Section 3.1.7). But it seems that the solubilization of MBBA induces considerable structural changes in the TTAB micelles and decreases the effective charge on the micelle. After $6.4 \mathrm{wt} \%$ MBBA in TTAB $25 \mathrm{wt} \%$ solution at $23^{\circ} \mathrm{C}$, the system becomes birefringent as shown in Fig. 14. The birefringence of the system increases with increasing MBBA concentration. Between 6.4 and $10 \mathrm{wt} \%$, there exists a temperature where the birefringent system turns non-birefringent ( $L_{1}$ phase). This 

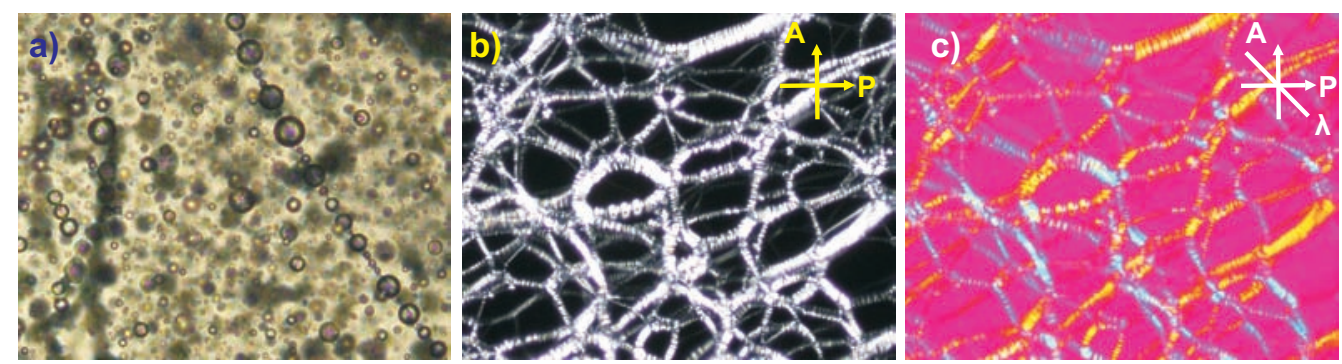

Figure 15: Optical microscopy images of LC-TTAB 25 wt\% equilibrium solutions at $23^{\circ} \mathrm{C}$. (a) Water in $5 \mathrm{CB}$ inverse emulsion phase. The continuous phase is $5 \mathrm{CB}$ in a nematic state and droplets contain aqueous TTAB solution. (b-c) The crossed polarizer and red wave plate images of the oily streak texture of $12 \mathrm{wt} \%$ MBBA in TTAB 25 wt\% solution.

transformation is also observed under the optical microscope. While heating the birefringent sample, the $L_{1}$ phase islands form and spread all over the sample at the transition temperature. While cooling many multi-lamellar vesicles (MLVs) and batonnets are spontaneously nucleated on entering the lamellar phase. MLVs show a maltese cross texture when viewed under crossed polarizers. After $14 \mathrm{wt} \%$ MBBA, the TTAB micelles cannot solubilize any more MBBA at $23^{\circ} \mathrm{C}$. Additional MBBA leads to the formation of nematic droplets just as in the case of $5 \mathrm{CB}$. However it is not easy to compare the properties of two compounds with very different molecular structures. Hence, the phase behavior of EBBA in TTAB 25 wt\% solution is investigated (Fig. 13(c)). The EBBA structure differs from MBBA by an extra methylene group added at one end of the chain (molecular chain length is $21 \AA$ ) [45]. The phase behavior of the EBBA system is very similar to $5 \mathrm{CB}$. No lamellar phase exists between the $L_{1}$ and the multiple emulsion phase. The saturation concentration of EBBA in TTAB $25 \mathrm{wt} \%$ solution is $4 \mathrm{wt} \%$ at $23^{\circ} \mathrm{C}$. This is in accord with the general trend of oil solubilization in micellar solutions. Increasing the molecular chain length reduces the solubilization limit in the micellar solutions [14]. Nor do any in the $\mathrm{nCB}$ series (2CB to $12 \mathrm{CB}$ ) produce any lamellar phase in TTAB $25 \mathrm{wt} \%$ solutions at or above $23^{\circ} \mathrm{C}$. Even here, as we increase the molecular chain length in the $\mathrm{nCB}$ series $(5 \mathrm{CB}, 8 \mathrm{CB}$ and $12 \mathrm{CB})$, the solubilization limit in the TTAB solutions decreases at any given temperature.

\subsubsection{Microscopy observations: Solubilization of NLCs in TTAB solutions}

There are two simple ways to perform solubilization experiments (Batch emulsion method, single droplet method). The Batch emulsion method carries a significant disadvantage if we want to understand the molecular pathways of solubilization. Two opposite physical phenomena are observed simultaneously: a decrease in droplet size and number density due to micellar solubilization, and an increase of the mean droplet size due to Ostwald ripening. Hence a single droplet in an aqueous micellar solution method is recommended as it simplifies the system considerably. Following this method, a large nematic parent droplet of the order of a few hundred microns is injected into a reservoir of TTAB micelles. The micellar solubilization of the parent droplet is tracked with a polarizing microscope equipped with a video recorder. 


\subsubsection{CB droplets}

When a $5 \mathrm{CB}$ droplet is immersed into a TTAB $25 \mathrm{wt} \%$ solution at room temperature, the droplet is in a nematic state and it spontaneously starts to self-propel itself in the aqueous micellar solution. Self-propulsion of the parent droplet is accompanied by convective flow inside and around the droplet. Convective flow inside a 5CB droplet is easily observed by the moving textural features (see Section 3.1.8) of the nematic phase under crossed polarizers. As we continue to observe the parent droplet under a microscope, one can start to recognize the evolution of some dirt-like objects inside the droplet. The size and number density of these objects ('daughter droplets') increase over time. Daughter droplets formed inside the parent $5 \mathrm{CB}$ droplet are probed with fluorescence confocal microscopy. This experiment confirms that daughter droplets are made up of the surrounding aqueous micellar solution, seeping through the 5CB - water interface (Fig. 16(a)).

Formation of stable aqueous daughter droplets inside a $5 \mathrm{CB}$ parent droplet requires TTAB at their interface. As TTAB is not soluble in $5 \mathrm{CB}$, the only way of transferring TTAB into a $5 \mathrm{CB}$ droplet is by means of inverted micelles. More and more inverted micelles

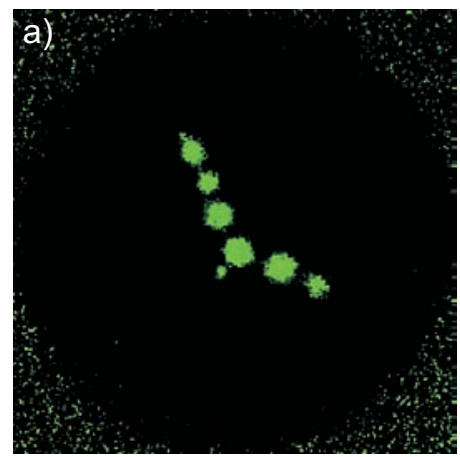

d)

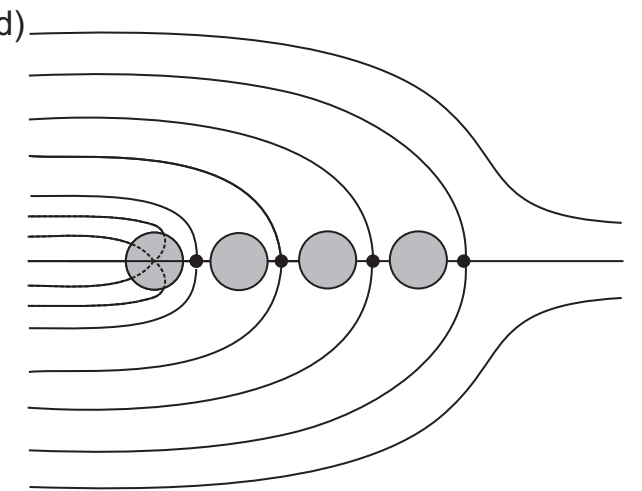

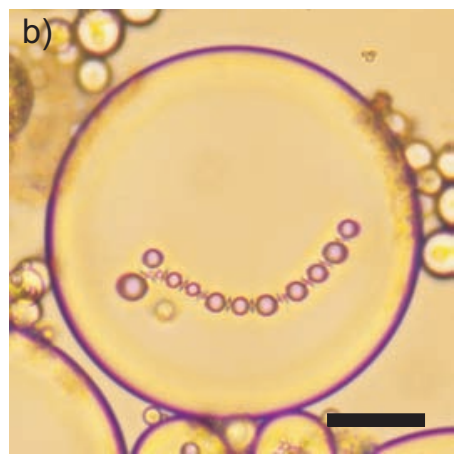
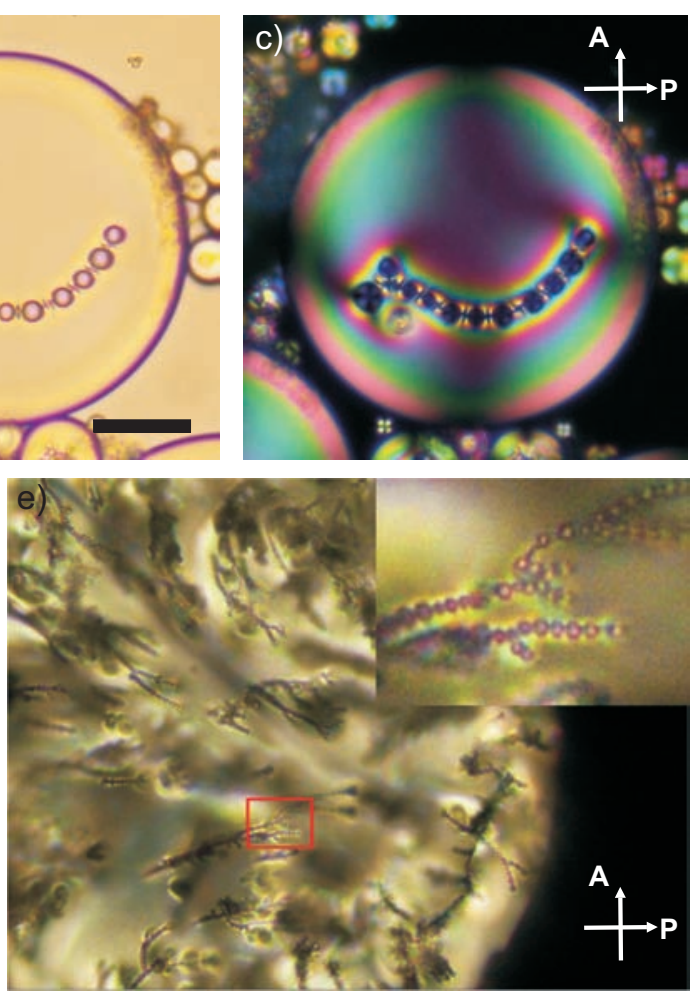

Figure 16: Aqueous daughter droplets in a 5CB parent droplet. (a) A 5CB droplet is initially immersed in a TTAB $25 \mathrm{wt} \%$ solution containing fluorescein. After the formation of daughter droplets, the parent $5 \mathrm{CB}$ droplet is transferred into a pure water solution. A strong fluorescence signal from the daughter droplets in the $5 \mathrm{CB}$ parent droplet clearly proves that they are made up of aqueous TTAB solution. The black circular region surrounding daughter droplets represents 5CB bulk. (b-c) White light and crossed polarizer images of a sandwiched 5CB droplet. Scale bar: $15 \mu \mathrm{m}$. (d) Schematic diagram of an aqueous water droplet chain in a nematic host. The big gray colored circles are water droplets and the small black circles are hyperbolic defects. The thin black lines represent the director orientation in the nematic host medium. (e) This image shows the formation of complex structures over time in a 5CB-TTAB $25 \mathrm{wt} \%$ system. Inset shows a larger magnification of the area marked by the red rectangle, whose horizontal width is $35 \mu \mathrm{m}$. 
seep into the $5 \mathrm{CB}$ droplet over time. This results in an increase in the number density of inverted micelles and in the frequency of collisions between them. These collisions result in the merging of inverted micelles which grow in size over time. This is due to the inability of single chain ionic surfactants such as TTAB and SDS to stabilize the inverted micelles in an oil (5CB) continuous phase. This process of coalescence does not continue indefinitely but stops after daughter aqueous droplets reach a critical size that is approximately a few micrometers. Then these aqueous droplets start to aggregate in the form of linear chains (Fig. 16(b-d)). These linear chains may also evolve over time into large complex structures with lateral connections between chains (Fig. 16(e)). Similar behavior is observed during the phase separation of a binary mixture [47]. This binary mixture consists of an isotropic fluid (silicone oil) and a liquid crystal (E7). A small amount of silicone oil in E7 forms a single nematic phase at $50^{\circ} \mathrm{C}$. Rapid cooling to room temperature nucleates small silicone oil droplets, which grow in size until they reach a critical size of a few micrometers. Then these silicone droplets start forming linear chains just as in the current system. These specific interactions are a result of orientational elasticity of the continuous phase. The energy scale of an elastic distortion around a spherical particle with radius " $a$ " is of order " $K a$ ", assuming a single elastic constant $K$, for splay, twist and bend deformations, whereas surface anchoring energy of the corresponding spherical particle amounts to $W a^{2}$. The constant $W$ is known as molecular anchoring strength, which is a measure of the ease with which orientation at the interface can deviate from its preferred anchoring direction. Strong homeotropic anchoring is achieved at the aqueous droplet interface only when surface anchoring energy becomes comparable to the energy scale of the elastic distortion around the droplet (i.e., above a critical radius where $W a^{2} \sim K a$ ). Thus, small aqueous droplets (i.e., with radii below the critical value) can freely diffuse and possibly coalesce when they collide with each other, as they do not induce significant distortions in the surrounding NLC medium. A droplet with strong homeotropic anchoring is topologically equivalent to a radial hedgehog defect of topological charge +1 . A hyperbolic hedgehog defect with opposite topological charge -1 becomes nucleated near the surface of the aqueous droplet as shown in Fig. 16(d) [47-51]. Both these defects combine and produce a net charge of zero. This defect pair behaves like an electrostatic dipole at the long range. Thus droplets attract each other at long range but the presence of a topological defect between two approaching droplets induces a short range repulsion. This explains the formation of aqueous droplet chains. These attractive interactions are much greater than the entropic free energy of the aqueous droplets [48]. As a result, chains are stable against thermal fluctuations. However, due to the convective flow inside the parent $5 \mathrm{CB}$ droplet, these aqueous droplet chains may disassemble and assemble repeatedly with or without the same bunch of aqueous droplets. Even though micron-sized aqueous droplets do not coalesce with each other and form chains, each aqueous droplet in a chain can still continue to grow. This implies that inverted micelles in the surrounding $5 \mathrm{CB}$ medium can still merge with aqueous droplets in the chains.

Increasing the TTAB concentration in the surrounding aqueous medium increases the rate of formation and number density of aqueous daughter droplet chains inside the parent $5 \mathrm{CB}$ droplet. This clearly suggests that the rate of transfer of inverted micelles into a $5 \mathrm{CB}$ droplet increases with increasing TTAB concentration in the surrounding aqueous medium. Inverted TTAB micelles must be made out of TTAB molecules at the interface. This process is succeeded by the replenishing of the interface with TTAB molecules from the surrounding aqueous medium. The rate of replenishing depends on the TTAB micellar density around the droplet. Hence, it is conceivable that the rate at which aqueous daughter droplet chains form inside the parent $5 \mathrm{CB}$ droplet depends on the TTAB concentration in the surrounding aqueous medium. 


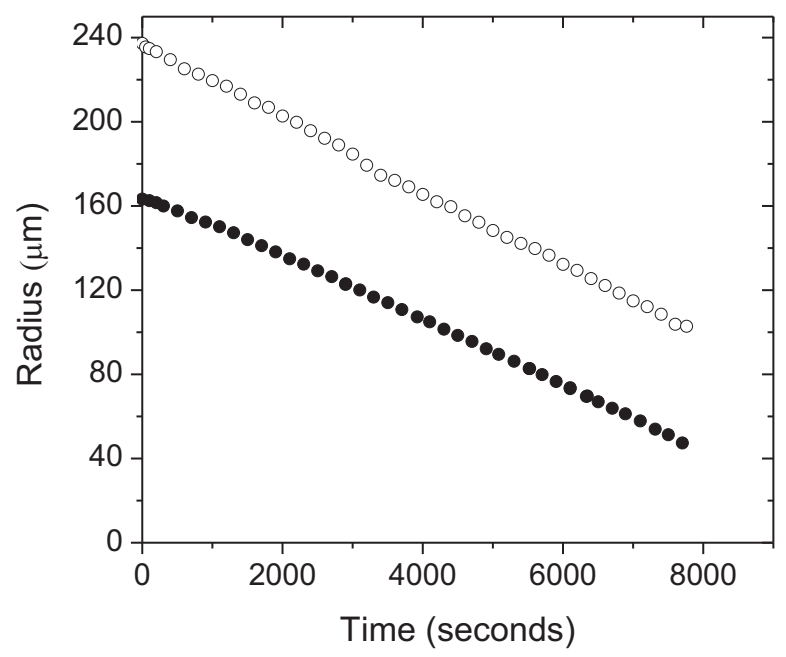

Figure 17: Rate of dissolution vs initial droplet size. Two significantly different sized 5CB droplets still dissolve at the same rate in TTAB $25 \mathrm{wt} \%$ solution at room temperature $\left(\sim 23^{\circ} \mathrm{C}\right)$.

Convective flows, disclination lines and aqueous daughter droplets in a $5 \mathrm{CB}$ parent droplet provide another kind of interaction. A recent report demonstrates that attractive interactions exist between disclination lines and aqueous droplets or colloids with normal anchoring in a flowing nematic host [52]. The only significant difference in the current system is that flow inside the nematic host (i.e., parent droplet) is not artificially generated. A single dipolar droplet or dipolar droplet chains produced in a parent $5 \mathrm{CB}$ droplet become attracted toward the disclination lines and position themselves along the disclination lines. The distance between dipolar aqueous droplets (initially in a chain) before and after merging with a disclination line remains intact. Convective flow inside the parent $5 \mathrm{CB}$ droplets can drag the trapped aqueous droplets along the disclination line. If the convective flow is perpendicular to the disclination line, trapped aqueous droplets can be freed from it.

Despite the continuous mass transfer from the aqueous micellar solution into the $5 \mathrm{CB}$ parent droplet, its radius $R$ decreases over time. This implies that there exists a much more significant continuous mass transfer of $5 \mathrm{CB}$ into the aqueous micellar solution as well. As the $5 \mathrm{CB}$ molecular solubility in pure water is very limited $(0.07 \mathrm{mg} / \mathrm{L}), 5 \mathrm{CB}$ molecules must be solubilized in the TTAB micelles. Unlike the formation of aqueous daughter droplets inside the $5 \mathrm{CB}$ parent droplet, no such $5 \mathrm{CB}$ daughter droplets form in the surrounding aqueous medium. After several hours, the 5CB droplet is completely solubilized and a viscous optically clear solution remains. The radiuses of the $5 \mathrm{CB}$ droplets decrease linearly over time, in the investigated range of sizes. Fig. 17 demonstrates that the rate of solubilization is independent of the initial size of the $5 \mathrm{CB}$ droplet for a given concentration of TTAB.

\subsubsection{Solubilization of 5CB vs Temperature}

When the same experiment is conducted at higher temperatures at which the $5 \mathrm{CB}$ droplet is in an isotropic state, a similar behavior is observed. The radius of the $5 \mathrm{CB}$ droplet still decreases linearly but at a faster rate of solubilization (over time, aqueous daughter droplets do start to emerge in the isotropic parent $5 \mathrm{CB}$ droplet, as in the case of the $5 \mathrm{CB}$ droplet in the nematic phase (Fig. 18(a, c)). Aqueous daughter droplet size and number density increase over time as is the case for 5CB nematic droplets. However, these aqueous daughter droplets do not form chains (in the isotropic 5CB droplet) as they do in the case 
a)

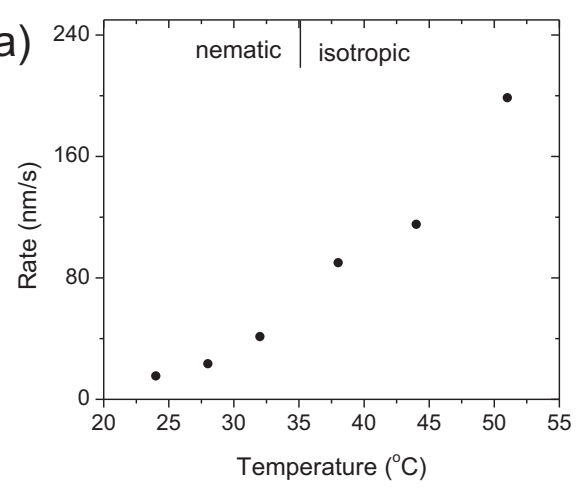

b)

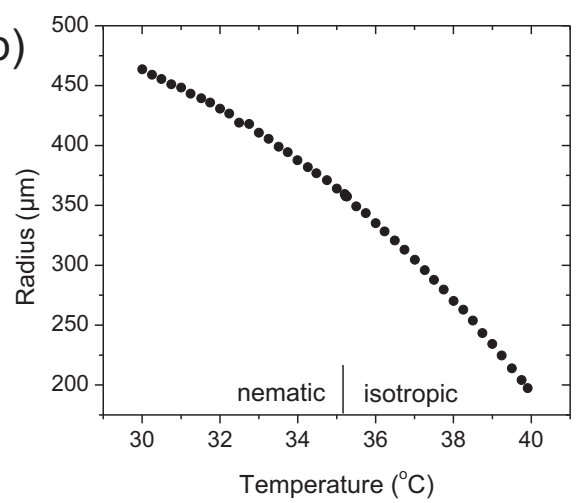

c)

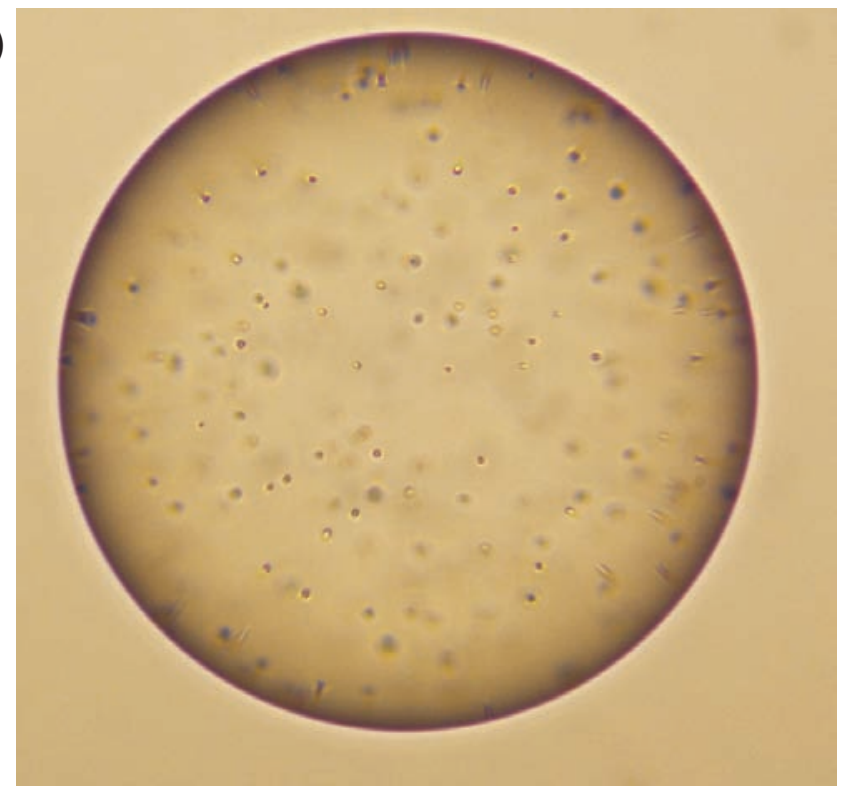

Figure 18: Effect of temperature on 5CB droplet solubilization rates. (a) The rate of solubilzation of the $5 \mathrm{CB}$ droplet in TTAB $25 \mathrm{wt} \%$ solution increases with increasing temperature. (b) Solubilization of a 5CB droplet in TTAB $25 \mathrm{wt} \%$ solution during a constant temperature ramp. This experiment shows that the Nematic - Isotropic transition does not have any significant effect on the solubilization rates. (c) The 5CB isotropic droplet in a TTAB $6 \mathrm{wt} \%$ micellar solution at $45^{\circ} \mathrm{C}$ after five minutes. Point like objects inside the droplet are indeed aqueous daughter droplets. It is quite clear that no aqueous daughter droplet chains form inside an isotropic 5CB droplet. Horizontal width of the image is $410 \mu \mathrm{m}$.

of the nematic 5CB droplet. Fig. 18(b) shows that the presence of an extra molecular order in the parent $5 \mathrm{CB}$ droplet (nematic) does not seem to have any prominent influence on the solubilization rates. But this is not entirely true in every system. In certain cases, nematic liquid crystal droplets do solubilize faster at lower temperatures in ionic surfactant micellar solutions. This is not observed in the case of the same droplets in the isotropic state. Parent droplets in the isotropic state always solubilize faster at higher temperatures in ionic micellar solutions. One example of the anomalous faster solubilization at lower temperatures in nematic droplets is provided in Section 3.1.4.2.

\subsubsection{Solubilization of $5 \mathrm{CB}$ vs TTAB concentration}

The critical micellar concentration $(\mathrm{CMC})$ for $\mathrm{TTAB}$ is $0.13 \mathrm{wt} \%$ at $25^{\circ} \mathrm{C}$ (CMC increases slowly with increasing temperature as the molecular solubilization of TTAB molecules in 
water increases). Below the CMC, only the molecular solubilization of $5 \mathrm{CB}$ is possible as there are no micelles available. As a result, the solubilization rate is very low $(-\mathrm{dR} / \mathrm{dt}=$ $1 \mathrm{~nm} / \mathrm{s}$ at $30^{\circ} \mathrm{C}$ and $0.08 \mathrm{wt} \%$ TTAB concentration). The solubilization rate of $5 \mathrm{CB}$ is independent of the TTAB concentration $\left(C_{s}\right)$ until $C_{s}$ reaches CMC. There is a complete absence of self-propelled motion of $5 \mathrm{CB}$ droplets as well as of convective flows inside and around them for $C_{s}<\mathrm{CMC}$. However, due to the finite molecular solubilization of water in $5 \mathrm{CB}$, aqueous daughter droplets can be observed even for $C_{s}<\mathrm{CMC}$. A similar observation is made for $5 \mathrm{CB}$ films in contact with pure water [53]. 5CB droplet solubilization becomes much more pronounced for TTAB concentrations, $C_{s}>$ CMC through micellar solubilization. The solubilization rate of $5 \mathrm{CB}$ droplets increases nonlinearly with increasing TTAB concentration. More details about this nonlinear dependence are provided in Section 3.1.5. Self-propelled motion of the 5CB droplet and convective flows inside and around the droplet increase significantly with increasing TTAB concentration.

\subsubsection{Effect of solubilizate chain length on solubilization rates}

Three different LCs $(5 \mathrm{CB}, 6 \mathrm{CB}$ and $7 \mathrm{CB})$ in the $\mathrm{nCB}$ series are considered. All three LCs are in the nematic state at room temperature. Increasing the solubilizate chain length results in a decrease in the solubilization rate at a given TTAB concentration. As shown in Fig. 19, this relationship between solubilizate chain length and solubilization rate holds true irrespective of the parent droplet state (i.e., nematic or isotropic).
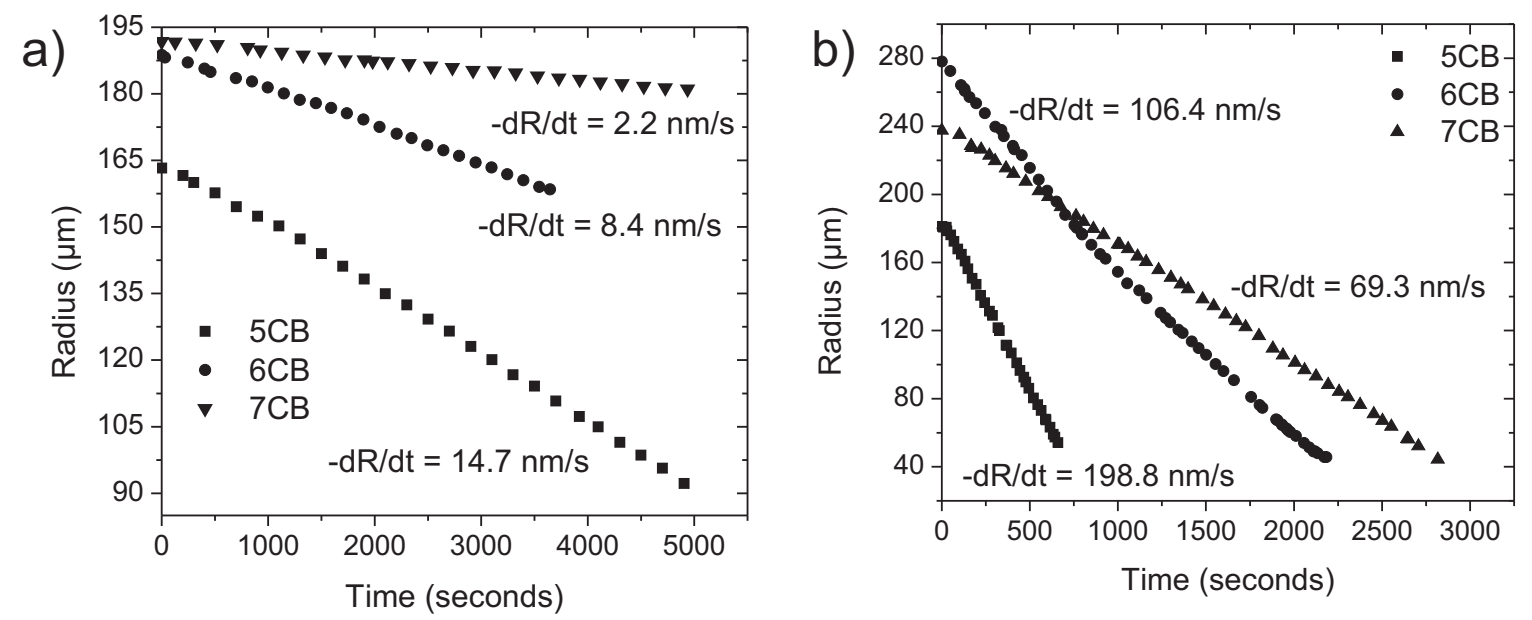

Figure 19: Chain length dependence on solubilization kinetics. (a) Increasing the chain length of the solubilizate decreases the solubilization rate in TTAB $25 \mathrm{wt} \%$ solution at $24^{\circ} \mathrm{C}$ (b) The same chain length dependence is observed for LCs in TTAB $25 \mathrm{wt} \%$ solution at $64^{\circ} \mathrm{C}$. The temperature effect on solubilization kinetics of each LC is also the same. The rate of solubilization increases with increasing temperature irrespective of the droplet state (i.e., nematic or isotropic). 


\subsubsection{MBBA droplets}

MBBA behaves differently in the nematic phase. For a given concentration of TTAB, there exists a temperature below which the parent MBBA droplet starts ejecting tiny daughter MBBA droplets spontaneously into the surrounding aqueous phase (Fig. 20(a)). The diameters of these tiny droplets are of order $1 \mu \mathrm{m}$. These tiny droplets further dissolve into the surfactant solution completely and thus an optically clear solution is obtained after some time (in the order of a few minutes to a few hours). If a daughter MBBA droplet is not crowded by other daughter droplets, then it quietly disappears over time. If the ejected daughter MBBA droplets are very near to each other ( $1 \mu \mathrm{m}$ or less), they form a transient Lyotropic LC phase by mixing with the surrounding TTAB solution. The resulting ma-

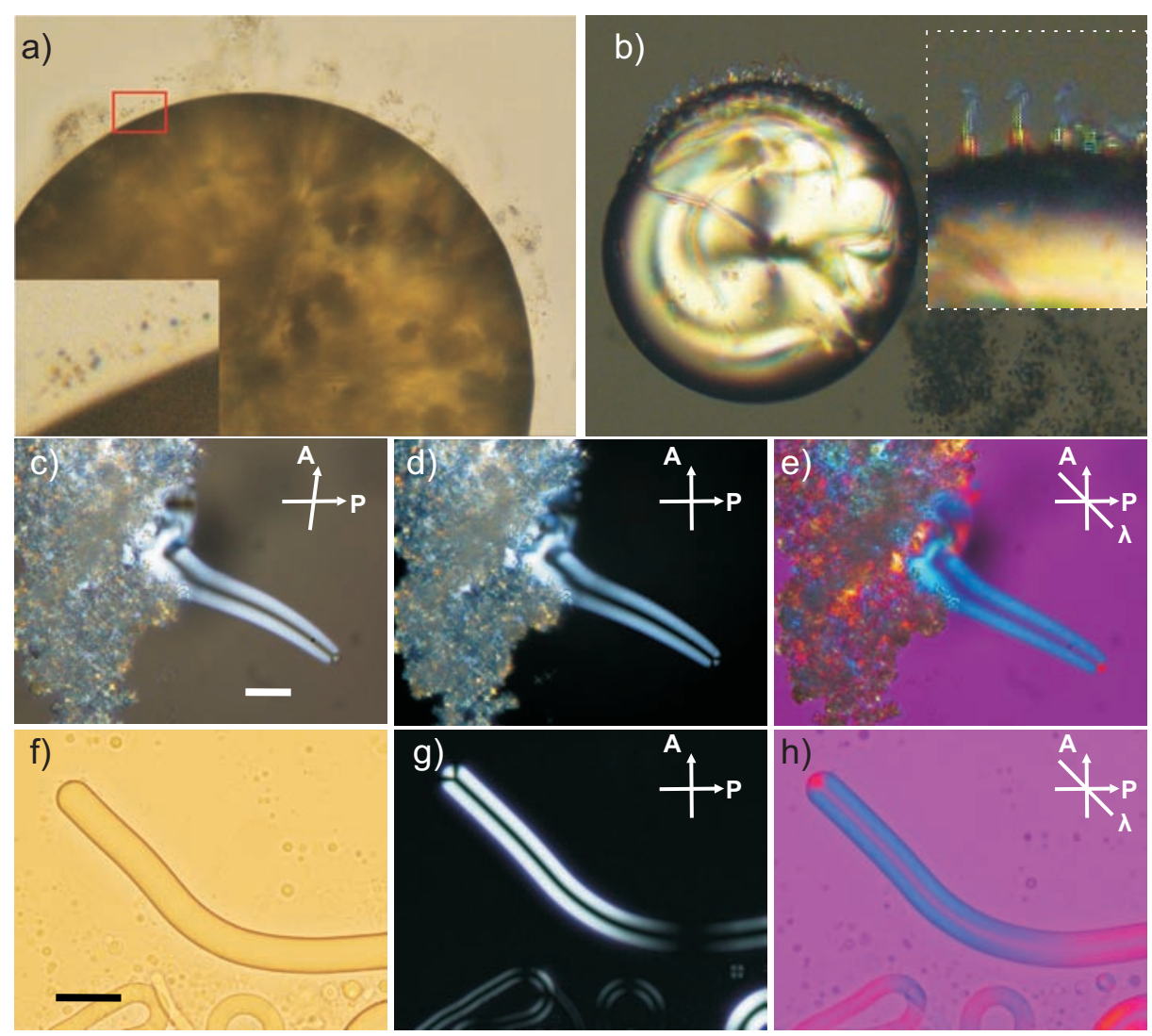

Figure 20: Solubilization process of MBBA in TTAB solution. (a) The MBBA parent droplet expels tiny daughter MBBA droplets in TTAB $10 \mathrm{wt} \%$ solution at $23^{\circ} \mathrm{C}$. The inset shows a larger magnification of the marked area by a red rectangle (horizontal width $70 \mu \mathrm{m}$ ). (b) A $100 \mu \mathrm{m}$ MBBA droplet in a TTAB $7.5 \mathrm{wt} \%$ at $23^{\circ} \mathrm{C}$. Microjets are clearly visible along the interface. Inset shows magnified image of microjets. (c-e) If the expelled droplets are very near to each other, they form a lyotropic lamellar phase with TTAB $10 \mathrm{wt} \%$ solution. Pictures c-e show polarization microscopy images of an MBBA filament growing out of the lamellar region. Scale bar in the image b represents $25 \mu \mathrm{m}$. (f-h) In order to show the similarity of the MBBA filament with classical myelin figures, the $C_{12} E_{3}$ - water system is chosen. Pictures f-h show the polarizing microscopy images of myelin figures growing from the $C_{12} E_{3}$ - water interface into an aqueous medium. Structurally, MBBA filaments are almost identical to classical myelin figures except that the tube diameter is not constant in the case of the MBBA filament. More structural details of these kind of cylindrical objects are provided in Chapter 4. Scale bar in image e represents $50 \mu \mathrm{m}$. A, P and $\lambda$ symbols indicate the analyzer, polarizer and red wave plate configurations applied for taking pictures. 
terial has a lamellar structure (Fig. 20(c)). Owing to its lamellar structure, this material forms transient lyotropic myelin-like figures (MBBA filament, Fig. 20(c-e). These MBBA filaments are different to the myelinic figures formed by systems such as $C_{12} E_{3}$ - water. In the case of classical systems like $C_{12} E_{3}$ - water, the myelinic figures that grow from the $C_{12} E_{3}$ - water interface are in an almost perfect cylindrical shape as shown in Fig. 20(f-h) [54]. It is reported that there is no significant mass transfer between the aqueous medium and the myelinic figure across the surface of the myelinic figure $[55,56]$. However, in the present case, the MBBA filaments rarely have a perfect cylindrical shape. The cross-sectional diameter of the filaments continuously decreases from the root to the tip. This clearly suggests that there is a significant mass transfer across the MBBA filament and aqueous micellar solution interface. We have not observed similar behavior in the 5CB-TTAB-water system at or above room temperature $\left(23^{\circ} \mathrm{C}\right)$ for TTAB concentrations up to $25 \mathrm{wt} \%$. This may be due to the fact that the MBBA-TTAB-water system can form an equilibrium lamellar phase, whereas the 5CB-TTAB-water system does not form lamellar phases at or above room temperature (see Section 3.1.1). Please note that we restricted ourselves to TTAB $25 \mathrm{wt} \%$ in the TTAB-water system as we wanted to work only with low viscosity aqueous TTAB micellar solutions $\left(L_{1}\right.$, solution phase). Preliminary experiments show that even $5 \mathrm{CB}$ droplets expel tiny daughter $5 \mathrm{CB}$ droplets well below room temperature $\left(\sim 5^{\circ} \mathrm{C}\right)$ in TTAB $25 \mathrm{wt} \%$ solution. Similarly, all the different NLC droplets tested eject tiny daughter NLC droplets under certain specific conditions of temperature, surfactant concentration, and additives such as salts. This shows that the ejection of daughter NLC droplets is a universal behavior of all NLCs.

\subsubsection{Spontaneous expulsion of daughter MBBA droplets - Threshold temperature $\left(T_{c}\right)$}

The threshold temperature below which the parent MBBA droplet expels daughter MBBA droplets can be manipulated by changing the concentration of surfactant in the surrounding aqueous micellar solution. As shown in Fig. 21, the temperature at which the ejection starts $\left(T_{c}\right)$ increases with increasing surfactant concentration. Apart from the expulsion

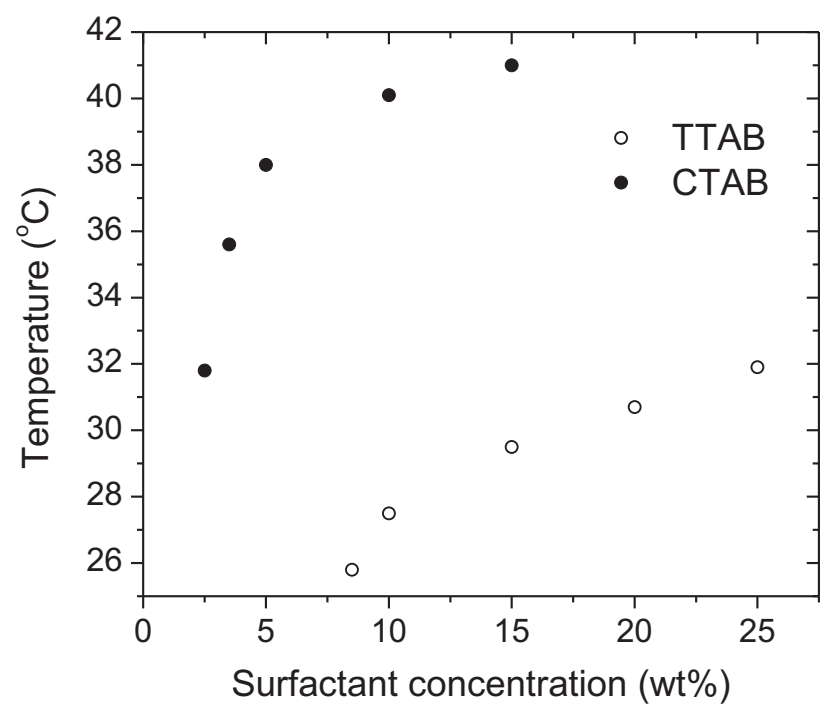

Figure 21: Effect of surfactant concentration on threshold temperature. The threshold temperature $\left(T_{c}\right)$ below which the MBBA parent droplet expels daughter droplets is significantly higher for the CTAB solution than the TTAB solution at any given concentration. 
of the daughter MBBA droplet below $T_{c}$, MBBA droplets behave similarly in aqueous micellar solutions on either side of the threshold temperature. This includes the constant solubilization rate, convective flows, self-propelled motion and the formation of tiny aqueous daughter droplets inside the parent MBBA droplet.

Fig. 21 also shows threshold temperature dependence on the type of the surfactant present in the aqueous medium. TTAB and its higher homologue, CTAB, are used to investigate the expulsion behavior. It is well known that CTAB is much more efficient in reducing the interfacial tension in comparison to TTAB [14]. Efficiency of a surfactant is measured by the surfactant concentration required to achieve a certain interfacial tension. For a particular molar concentration of both surfactants, the threshold temperature obtained by CTAB is much higher than in the case of TTAB. These observations clearly suggest that a sudden drop in the interfacial tension exists at the threshold temperature, which facilitates the expansion of the interface without any need of external energy. The MBBA droplet surface area expands by forming jets (Fig. 20(b)). However, these jets are not stable and they instantly break up into tiny LC daughter droplets. Stable jets are also possible under certain conditions. More details about stable jets (or nematic filaments) are provided in Section 3.1.9. This morphological change of the MBBA droplet surface is a result of the competition between the bulk elastic energy and the surface energy of the MBBA droplets. It is noteworthy that threshold temperatures are always below the Nematic - Isotropic phase transition temperature of MBBA $\left(48^{\circ} \mathrm{C}\right)$ for all investigated surfactant concentrations.

\subsubsection{Solubilization of MBBA vs Temperature}

As explained in the above section, the solubilization of MBBA droplets follows two different routes depending upon whether the temperature of the system is above or below the threshold temperature. Fig. 22 shows the behavior of parent MBBA droplets in TTAB 10 wt\% solution at different temperatures. The threshold temperature for this current system

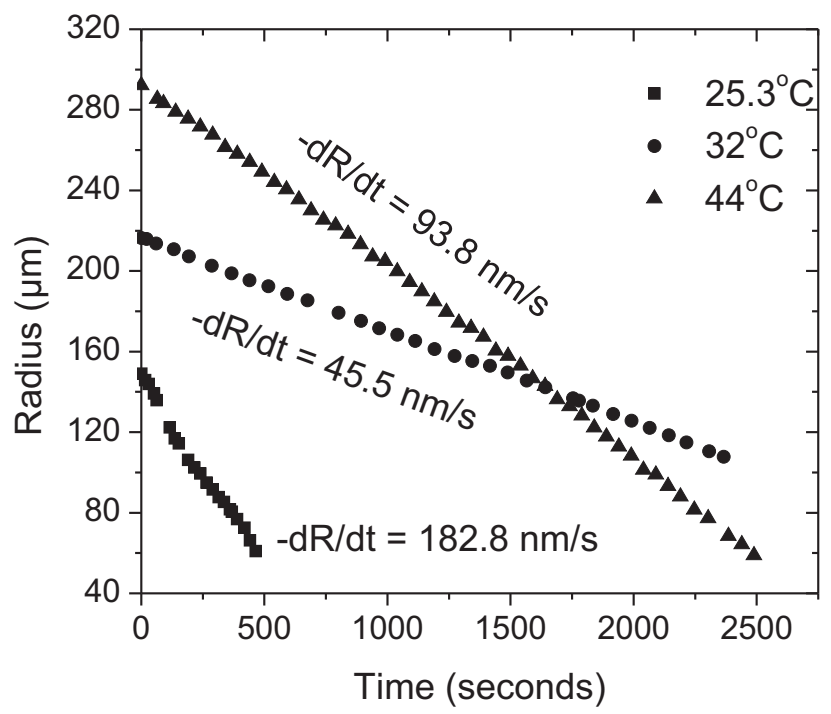

Figure 22: Solubilization rate of MBBA droplets vs Temperature. MBBA droplets are put into TTAB $10 \mathrm{wt} \%$ solutions. The threshold temperature is at $27.5^{\circ} \mathrm{C}$ for this system. Above the threshold temperature, the general behavior of solubilization is observed. The solubilization rate at $44^{\circ} \mathrm{C}$ is higher than the rate at $32^{\circ} \mathrm{C}$. But, an anomalous solubilzation rate value is observed for the system at $25.3^{\circ} \mathrm{C}$. This apparent high solubilization rate at lower temperature is due to the expulsion of daughter droplets by the parent MBBA droplet. 
is $27.5^{\circ} \mathrm{C}$. Above the threshold temperature, the solubilization rate of the MBBA droplet in the aqueous micellar solution increases with increasing temperature. This holds true even when the parent MBBA droplet is in an isotropic state. Due to the expulsion of the tiny daughter droplets from a parent MBBA droplet below $T_{c}$, the solubilization rates apparently become enhanced. Thus, as shown in Fig. 22, the solubilization rate of a MBBA droplet at $25.3^{\circ} \mathrm{C}$ is much greater than the solubilization rates at $32^{\circ} \mathrm{C}$ and $44^{\circ} \mathrm{C}$. It must be noted that the experimental line at $25.3^{\circ} \mathrm{C}$ in Fig. 22 represents the diameter of the parent MBBA droplet only. The expelled daughter droplets from the parent MBBA droplet take more time to solubilize completely in the aqueous micellar solution. However, the complete solubilization of the parent $\mathrm{MBBA}$ droplet at $25.3^{\circ} \mathrm{C}$ is still much faster than the solubilization at $32^{\circ} \mathrm{C}$ and $44^{\circ} \mathrm{C}$. As mentioned previously, this is one of the rare situations where the parent oil droplet solubilizes faster at lower temperatures in ionic surfactant micellar solutions. As we decrease the temperature (i.e., in the region below $T_{c}$ ), the rate of expulsion of daughter MBBA droplets increases significantly, but the time required for the complete solubilization of expelled daughter droplets in the aqueous micellar solution also increases significantly. Hence, within the region below the threshold temperature, $T_{c}$, the time required for the complete solubilization of the parent MBBA droplet decreases with increasing temperature. The existence of interfacial instability (i.e., droplet ejection) at lower temperatures in the nematic phase is in line with earlier reports $[57,58]$. These reports show that the interfacial tension of the nematic liquid crystal-aqueous ionic micellar solution interface decreases with decreasing temperature.

\subsubsection{Solubilization of MBBA vs TTAB concentration}

The rates of solubilization of parent MBBA droplets are investigated for different TTAB concentrations $(0.5-3 \mathrm{wt} \%)$ at $29^{\circ} \mathrm{C}$. MBBA droplets do not expel daughter droplets at these concentrations and this temperature. As shown in Fig. 23, the solubilization rate - $(d R / d t)$ non-linearly approaches towards zero as $C_{s}$ decreases. A comparison between the $5 \mathrm{CB}$ and the MBBA droplet dissolution rate, dependent on the TTAB concentration, is also presented in Fig. 23. The solubilization rate of MBBA droplets is at least two times

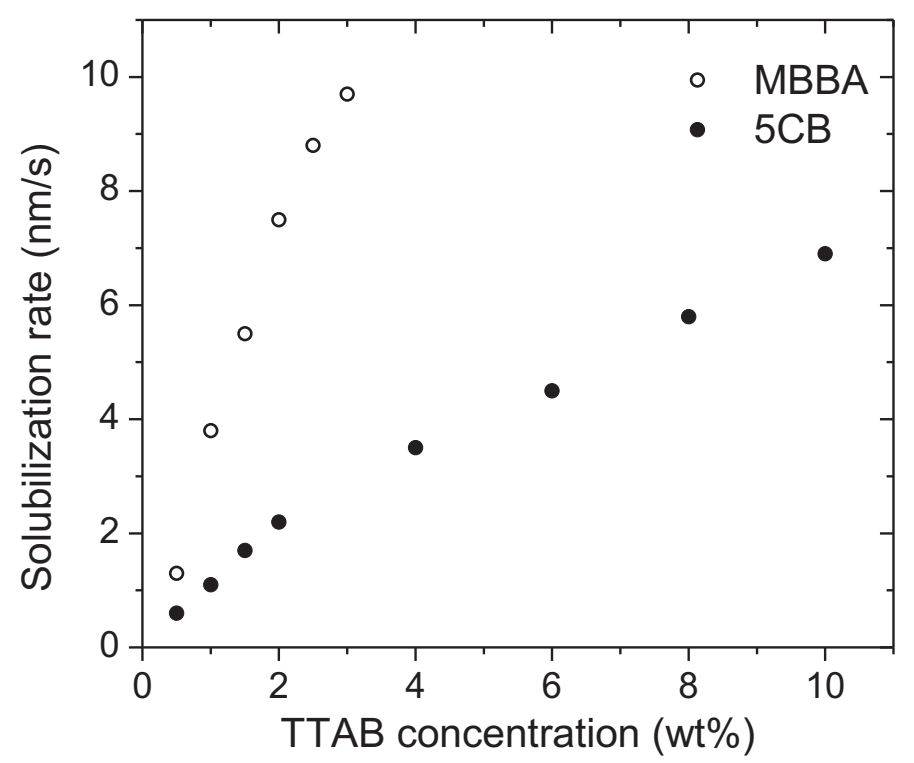

Figure 23: Rate of solubulization of $L C$ droplets vs TTAB concentration. Solubilization rate $-d R / d t$ is plotted as a function of TTAB concentration at a constant temperature $\left(29^{\circ} \mathrm{C}\right)$. 
higher than a $5 \mathrm{CB}$ droplet at any investigated TTAB concentrations. It is noteworthy that the molecular solubilization of MBBA in pure water $(5.6 \mathrm{mg} / \mathrm{L})$ is 80 times higher than $5 \mathrm{CB}(0.07 \mathrm{mg} / \mathrm{L})$ at $25^{\circ} \mathrm{C}$. The possible relationship between molecular solubilization in pure water and micellar solubilization is discussed in the following section.

\subsubsection{Plausible molecular pathways in the micellar solubiliza- tion of LC droplets}

Several mechanisms have been considered to explain the results of the micellar solubilization rates of organic liquids. Two mechanisms are generally accepted based on the type of the surfactant (ionic or non-ionic) and on the molecular solubility of the oil in pure water.

Ionic surfactants: In the case of ionic surfactants, the oil droplet - water $(\mathrm{O} / \mathrm{W})$ interface and micelles repel each other due to electrostatic repulsion. Individual oil molecules from a parent oil droplet dissolve in the aqueous phase initially. These solubilized oil molecules diffuse away from the $\mathrm{O} / \mathrm{W}$ interface and are subsequently captured by micelles $[33,37,38,40]$. Depending upon the molecular solubility and the concentration of the surfactant, individual oil molecules become incorporated into micelles within the diffusion boundary layer or after they enter the bulk aqueous solution. This molecular pathway generally explains the behavior of oils which have considerable molecular solubility in water. However, the case of extremely insoluble oils like triglycerides cannot be explained with this mechanism. A schematic diagram of this plausible mechanism (i.e., molecular pathway) for ionic surfactants is provided in Fig. 24(a).

Non-ionic surfactants: Due to the lack of electrostatic repulsions, micelles can directly adsorb onto the parent oil droplet. But it is very well known that micelles are not particularly surface active. Thus, realistically, micelles dissociate into separate surfactant molecules (monomers) in the diffusion boundary layer. The dissociation of micelles or the demicellization process is expected to happen through the step-by-step removal of monomers from the micelles rather than through the one step disintegration of micelles. These dissociated individual surfactant molecules adsorb onto the $\mathrm{O} / \mathrm{W}$ interface due to their strong surface activity. Adsorbed excess surfactant molecules are subsequently detached from the $\mathrm{O} / \mathrm{W}$ interface in the form of micelles fully or partially filled with oil molecules from the parent oil droplet (Fig. 24(b)). The molecular pathway can also still play a significant role along with this micelle-mediated pathway in solubilizing the oil droplets in a non-ionic surfactant solution, if the molecular solubility of oil in water increases significantly [35-37].

Our experiments are restricted to LC droplets in aqueous ionic micellar solutions. LC droplets in non-ionic surfactants are not a suitable system for us as our goal is to understand the effect of the nematic order on the solubilization behavior of oil droplets in aqueous micellar solutions. Non-ionic surfactants are soluble in both water and LCs to some extent. When a pure nematic droplet is introduced into a non-ionic surfactant solution, surfactant molecules are redistributed between the parent LC droplet and the water medium. This inevitable reorganization of non-ionic surfactants may cause the parent LC droplets to lose their nematic order and turn into isotropic droplets over time. Thus we restricted ourselves to ionic surfactants whose molecular solubility in oils is negligible.

Based on the solubilization results obtained for decane and benzene in SDS solutions, Todorov et al. developed a theoretical model that describes the solubilization of a single oil droplet in an aqueous ionic micellar solution [40]. A difference of five orders of magnitude exists between the molecular solubility of decane $(65 \mu \mathrm{g} / \mathrm{L})$ and benzene $(0.94 \mathrm{~g} / \mathrm{L})$ in pure water. This difference in molecular solubility in water has a profound effect on the solubilization rates. While the decane droplet radius decreases linearly over time, the benzene droplet 


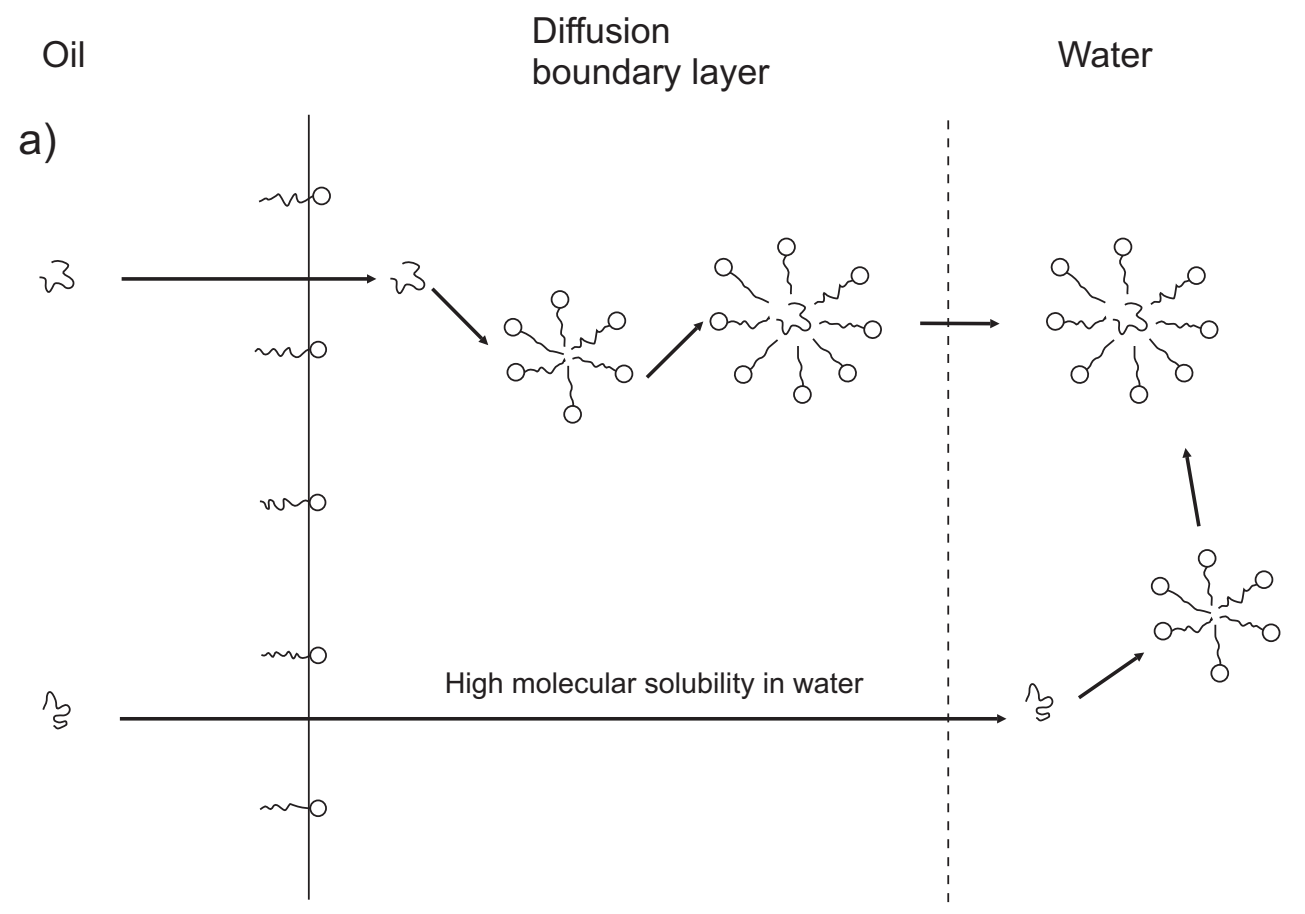

b)

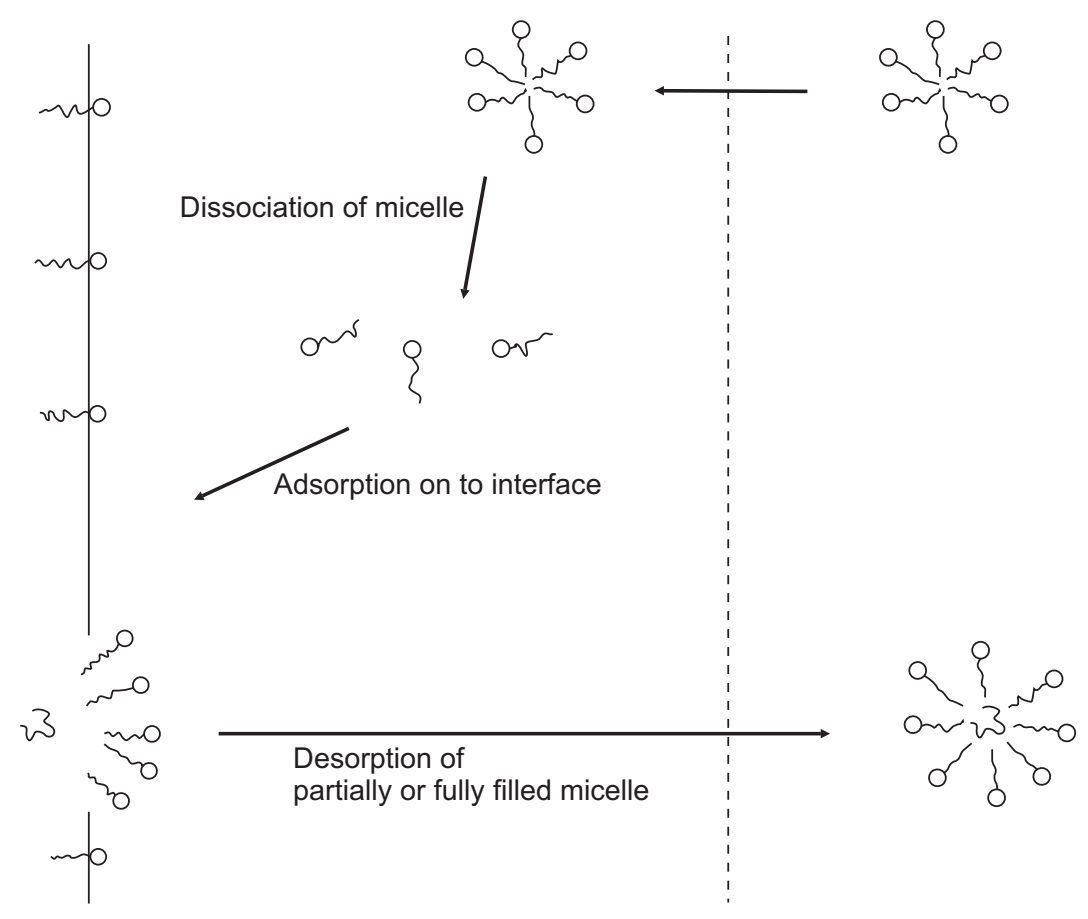

Figure 24: Schematic diagram of plausible oil solubilization pathways. (a) Molecular pathway. If the solubility of oil in water is very high, oil droplets dissolve molecularly in the bulk water solution at first and later micelles may capture oil molecules solubilized in bulk water. If the molecular solubility of oil in water is very low, micellar solubilization only occurs in a small region around the oil droplet called the diffusion boundary layer or solubility zone. (b) Micelle mediated pathway. In the case of high hydrophobic oils, solubilization follows this path in non-ionic surfactant solutions.

radius decreases non-linearly over time (the rate of solubilization increases over time). In SDS $0.25 \mathrm{M}$ solution, the rate of solubilization of benzene droplets is $>70 \mathrm{~nm} / \mathrm{s}$, whereas the rate of solubilization of decane droplets is very low, $\sim 2 \mathrm{~nm} / \mathrm{s}$. The molecular solubility 
of investigated LC molecules (5CB, $70 \mu \mathrm{g} / \mathrm{L} ;$ MBBA, $0.56 \mathrm{mg} / \mathrm{L})$ in water is comparable to that of decane and all these three organic liquid droplets show a linear decrease in the size of droplets over time. Thus, Todorov's decane droplet solubilization model is applied to the LC droplets.

\subsubsection{Todorov's theoretical model}

The basic assumption of the model is that the concentration of full micelles (i.e., a full micelle cannot accommodate any more new oil molecules) within the diffusion boundary layer is zero. This means micellar diffusion is sufficiently faster than the micellar solubilization of oil molecules. Active micelles leave the diffusion boundary layer before they become full micelles. It is also assumed that oil molecules diffuse into the aqueous medium and are then captured by active micelles within the diffusion boundary layer (solubility zone). For sparingly soluble oil like decane, it is quite reasonable to assume that the characteristic length of the solubility zone $\left(\kappa^{-1}\right)$ is very small in comparison to the radius of the droplet at any given moment $(\mathrm{R})$ during the entire length of the experiment. Concentration of active micelles $\left(c_{a}\right)$ within the solubility zone is assumed to be constant and equal to $\frac{c_{s}}{n_{m}}=\frac{c-c m c}{n_{m}}$. Here $\mathrm{c}$ stands for surfactant concentration in the bulk solution, and cmc is the critical micellar concentration of the surfactant and $n_{m}$ is the mean aggregation number of the micelles.

For all the above conditions, the local concentration of oil molecules in the aqueous medium $\left(c_{o i l}\right)$ obeys the equation

$$
\begin{array}{r}
\nabla^{2} c_{o i l}=\kappa^{2} c_{o i l}, \\
\kappa^{2}=\frac{k_{+} c_{a}}{D_{o i l}},
\end{array}
$$

here $D_{\text {oil }}$ stands for the diffusivity of oil molecules dissolved in water and $k_{+}$for the rate constant of solubilization. The imposed boundary conditions for solving Eq. 4 in spherical coordinates are: no oil in the micelles at the infinity and no mass flux of the micelles into the parent oil droplet.

$$
-\left.\frac{\partial c_{o i l}}{\partial r}\right|_{r=R}=\delta\left(c_{e q}-c_{o i l}(R)\right),\left.c_{o i l}\right|_{r=\infty}=0,
$$

where $\mathrm{r}$ is the radial distance from center of the oil droplet and $c_{e q}$ is the equilibrium solubility of oil in water. $\delta$ represents the mass-transfer coefficient across the O/W interface. By solving Eq. 4 for conditions of Eq. 5 and Eq. 6 results in the following equation

$$
c_{\text {oil }}(r)=\frac{c_{e q} R}{1+\left[\frac{\kappa+R^{-1}}{\delta}\right]} \frac{e^{[\kappa(R-r)]}}{r} .
$$

The number of oil molecules dissolved in water per unit area of the parent oil droplet surface and per unit time $\left(Q_{o i l}\right)$ is obtained with the help of Eq. 7 .

$$
Q_{o i l}=-\left.D_{o i l} \frac{\partial c_{o i l}}{\partial r}\right|_{r=R}=\frac{\kappa+R^{-1}}{1+\left[\frac{\kappa+R^{-1}}{\delta}\right]} D_{o i l} c_{e q}
$$

The rate of decrease in the volume of the parent oil droplet can be obtained by using Eq. 8.

$$
-\frac{d V}{d t}=v_{o i l}\left(4 \pi R^{2}\right) Q_{o i l}
$$


here $v_{\text {oil }}$ is the molar volume of the oil. Substituting the volume of the oil droplet $\left(V=\frac{4}{3} \pi R^{3}\right)$ and Eq. 8 in Eq. 9 gives the required expression for the rate of decrease in the radius of the oil droplet.

$$
\begin{array}{r}
\frac{d R}{d t}=-\frac{\delta \beta(1+\kappa R)}{1+(\delta+\kappa) R}, \\
\beta=v_{o i l} D_{o i l} c_{e q} .
\end{array}
$$

According to our initial assumption, the radius of the parent oil droplet at any given time $R$ is much larger than the characteristic length $\kappa^{-1}$ of the solubilization zone $(\kappa R>>1)$. In the limit $\kappa R>>1$, which corresponds to a narrow solubilization zone around the parent oil droplet, Eq. 10 can be approximated into the following form

$$
\begin{array}{r}
-\frac{d R}{d t} \approx \frac{\delta \beta \kappa}{\delta+\kappa} \equiv u \equiv \text { constant } \\
R(t)=R_{0}-u t .
\end{array}
$$

From Eq. 5 and Eq. 12 we can ascertain the relation between the rate of solubilization of oil droplets $(u)$ and the bulk surfactant concentration.

$$
\begin{array}{r}
u=\frac{a(c-c m c)^{0.5}}{b+(c-c m c)^{0.5}}=\frac{a c_{s}^{0.5}}{b+c_{s}^{0.5}}, \\
\text { where } a=\delta \beta, \quad b=\left[\frac{D_{o i l} n_{m} \delta^{2}}{k_{+}}\right]^{0.5} .
\end{array}
$$

According to Eq. 14, the rate of solubilization of oil droplets should vary as $c_{s}^{0.5}$ at small surfactant concentrations and approaches a constant value at high surfactant concentrations. This model represents the decane droplet micellar solubilization in SDS solution adequately [40].

Fig. 25 shows the Todorov's model fitting for the LC droplet (5CB and MBBA) dissolution data. Todorov's decane droplet model does not really fit the LC droplet dissolution experimental data. At small surfactant concentrations, Todorov's model overestimates the dissolution rates, whereas at high surfactant concentrations, the model significantly underestimates the LC droplet solubilization rates. However, if the power of $c_{s}$ in the Eq. 14 is treated as a variable instead of fixing it at 0.5 , excellent matches to the experimental observations can be obtained. Through this method, the obtained powers for $5 \mathrm{CB}$ and MBBA
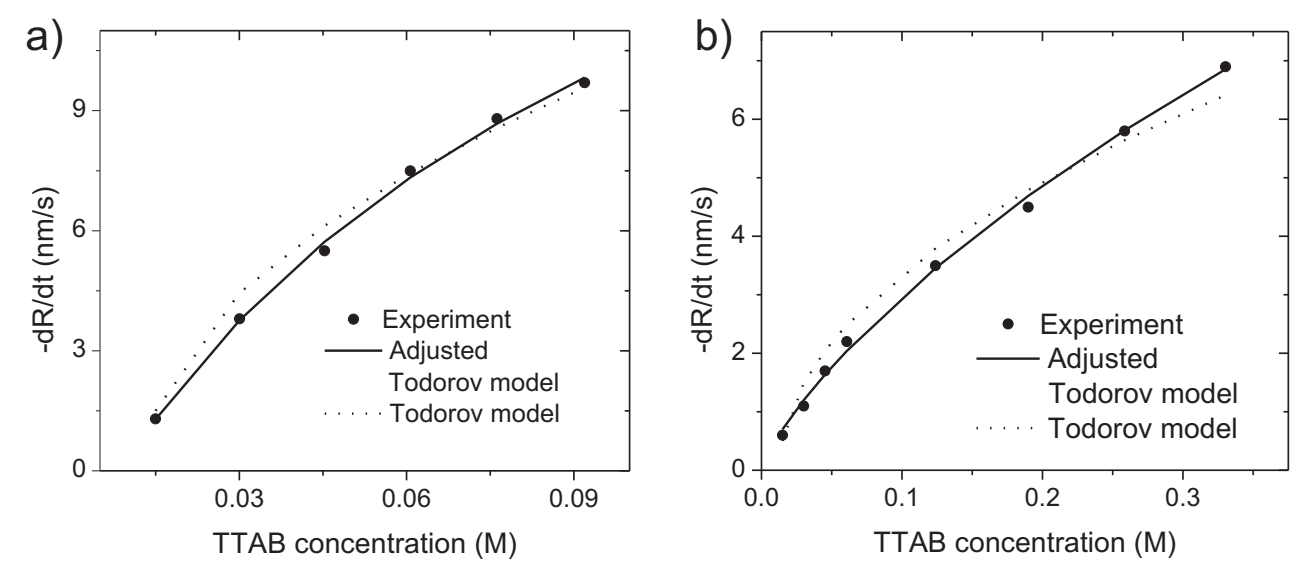

Figure 25: Model fittings for LC solubilization in TTAB solutions. (a-b) The rate of solublization varies non-linearly for both MBBA and $5 \mathrm{CB}$ with TTAB concentration. Model fittings are shown for MBBA (a) and 5CB (b) respectively. 
droplet solubilization in TTAB solutions are 0.79 and 0.96 respectively. This suggests that Eq. 4 and Eq. 5 may not be entirely correct for LC droplet micellar solubilization. Todorov's model assumes that active micellar concentration in the bulk and solubilization zone is the same and constant. This assumption holds true in the case of decane. However, this may not really apply for LC droplets, especially at small surfactant concentrations. Ionic surfactants do not diffuse into the parent decane droplet. But as we know, there is a continuous mass flux of surfactant monomers (individual molecules) into the parent LC droplet during the entire length of its solubilization period. Surfactant monomers at the LC droplet-water interface must continuously transform into inverted micelles and carry water in their hydrophilic cores. In order to replenish the interface continuously with surfactant monomers, active micelles entering into the solubility zone must dissociate into monomers partially or completely. This results in the decreasing density of active micelles in the solubility zone. If the active micellar concentration in the solubility zone is smaller than in the bulk solution, the apparent rate of solubilization goes down. This may explain the overestimation of solubilization rates by Todorov's model at small surfactant concentrations.

As the surfactant concentration increases, the influx of inverted micelles into the parent LC droplet also increases significantly. Thus, active micelles in the solubility zone must dissociate to replenish the interface continuously much more frequently than at the small surfactant concentration. But solubilization rates at high surfactant concentrations are higher than Todorov's model estimates. This may be explained through the possible formation of nematic nanojets at the LC droplet-water interface. As mentioned already, high surfactant concentrations and low interfacial tensions make the LC parent droplet-water interface very unstable and produce jets with several microns of cross-sectional diameter (microjets). The formation of jets greatly enhances the apparent rate of solubilization (see Section 3.1.4.2). It is conceivable to have nanojets (at the LC droplet interface) between the regions of molecular solubilization of oil and microjets, at intermediate surfactant concentrations. This could explain the differences between experimental results and estimations from Todorov's model for high surfactant concentrations. Attempts are being made in our lab to find out whether this nanojets hypothesis is indeed a true phenomenon. Similarly, extensive convections inside and around the parent oil droplets caused by Marangoni stresses may also affect the solubilization rates significantly at high surfactant concentrations. Convective flows inside and around the LC droplet increases significantly with increasing surfactant concentration.

\subsubsection{Rheology of LCs in TTAB solutions at equilibrium}

The incorporation of solubilizates into the micelles may induce significant changes in the micellar properties such as shape and size. There are several possible loci of solubilization in a micelle as shown in Fig. 26. The precise location at which solubilization occurs depends on the interaction between surfactant and solubilizate. The locus of solubilization in a micelle determines the extent of solubilization (solubilization capacity/power of the micelle) [14]. As mentioned in Section 3.1.1, MBBA forms single phase systems until $\sim 14 \mathrm{wt} \%\left(L_{1}\right.$ phase until $\sim 6.4 \mathrm{wt} \%$ ) in TTAB $25 \mathrm{wt} \%$ solution at $23^{\circ} \mathrm{C}$. However, $5 \mathrm{CB}$ forms single phase systems in TTAB $25 \mathrm{wt} \%$ until $\sim 3.5 \mathrm{wt} \%$ only at $23^{\circ} \mathrm{C}$. These differences in the solubilization capacity of TTAB micelles clearly suggest that the loci of solubilization are significantly different for 5CB and MBBA. Exact locations of solubilizates after solubilization in aqueous micellar solutions can be obtained using UV, NMR and fluorescence spectra [5964]. Changes in the micellar dimensions can be obtained through diffraction studies (SANS) [65-69]. The viscosity values of the TTAB micellar solutions saturated with either 5CB or MBBA are significantly higher than the viscosity values of pure TTAB solutions. Rheological 
a)

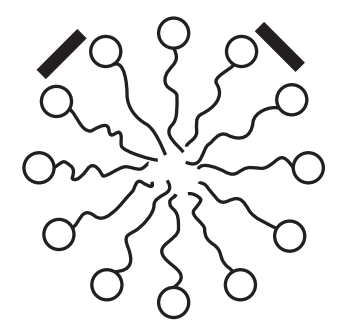

b)

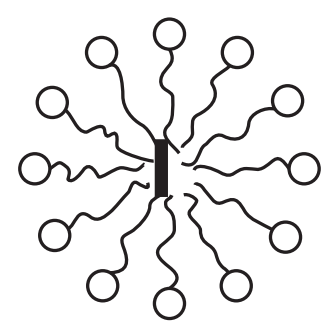

c)

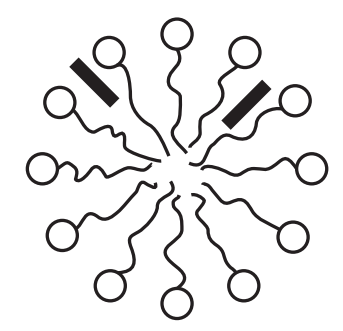

d)

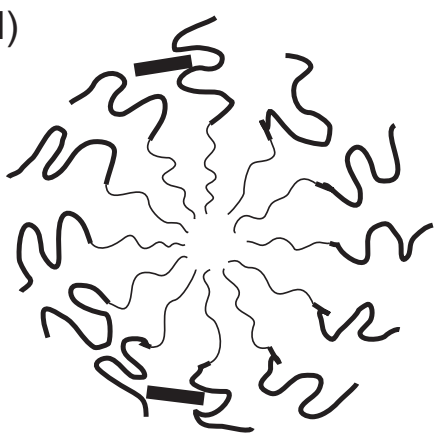

Figure 26: Possible loci of solubilization. The exact location of the solubilizate in a micelle depends upon the interaction between the solubilizate, surfactant monomers and the solvent. Solubilization can occur at four different sites in a micelle (a) at the micelle-solvent interface (b) in the inner core of the micelle (c) somewhere in the palisade layer (outer core) which consists of the hydrophilic head group and the first few carbon atoms of the hydrophobic group and (d) between the hydrophilic head groups of non-ionic surfactants. Closed circles represent the hydrophilic head groups of a surfactant. The black bold lines represent solubilizates. The light black curved lines represent surfactant tails and the black bold curved lines represent hydrophilic head groups of a non-ionic surfactant.

properties were investigated systematically for both 5CB-TTAB and MBBA-TTAB systems. The structural change of micelles induced by $5 \mathrm{CB}$ solubilization in TTAB samples were investigated by means of small-angle neutron scattering (SANS). SANS experimental data for 5CB-TTAB system is provided in Section 3.1.7.

\subsubsection{5CB in TTAB 25 wt\% solutions: Steady shear flow curves}

Fig. 27(a) shows flow properties for 5CB-TTAB solutions as a function of $5 \mathrm{CB}$ concentration at $23^{\circ} \mathrm{C}$. Pure TTAB $25 \mathrm{wt} \%$ solution showed a Newtonian behavior within the region of investigated shear rates. Pure TTAB 25 wt\% obeys Newton's postulate (Eq. 13) which states that the force resisting the motion of liquids is proportional to the range of applied
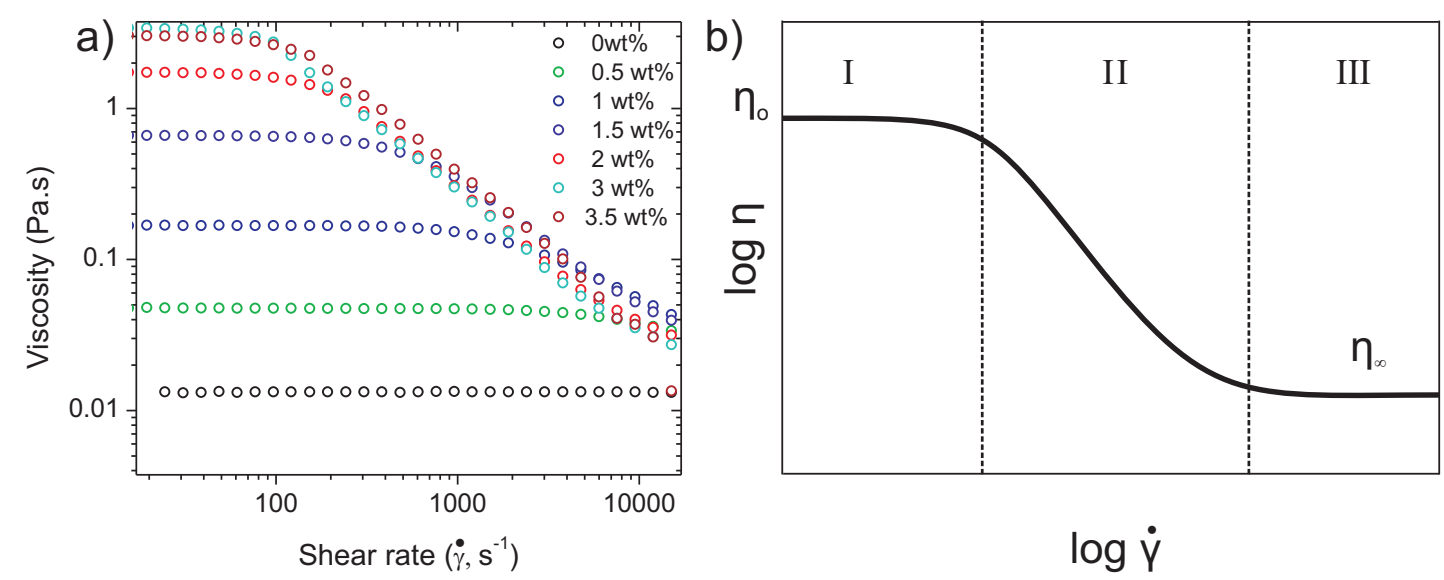

Figure 27: Steady shear flow curves of $5 C B$ in TTAB 25 wt\% solutions. (a) Steady shear flow curves as a function of $5 \mathrm{CB}$ concentration in the $(0-3.5)$ wt\% range at $23^{\circ} \mathrm{C}$. (b) Generalized flow equilibrium curve of non-Newtonian fluids. Region I is the first Newtonian plateau, Region II is the shear thinning (power law) regime and Region III is the second Newtonian plateau. 
shear rates (ideal viscous flow behavior).

$$
\tau=\eta \dot{\gamma}
$$

$\tau, \dot{\gamma}$ and $\eta$ represent shear stress, the shear rate applied to the liquid and the viscosity of the liquid respectively. All the investigated 5CB-TTAB mixtures exhibit Newtonian behavior at a low shear rate regime. However, 5CB-TTAB mixtures do not obey Newton's law over the total range of investigated shear rates (non-Newtonian behavior). After a certain critical shear rate, apparent viscosity decreases with increasing applied shear rate for each 5CBTTAB mixture. Fig. 27(b) shows a generalized equilibrium flow curve which represents the shear rate against viscosity for non-Newtonian fluids. This figure consists of a low shear rate Newtonian regime (Region I), an exponential shear thinning regime (Region II) and a high shear Newtonian regime (Region III). Shear thickening and time-dependent behaviors of liquids are ignored here. Several model functions are available for describing the equilibrium flow curves including zero-shear $\left(\eta_{0}\right)$ and infinite-shear viscosities $\left(\eta_{\infty}\right)$.

$$
\begin{aligned}
\text { Zero shear viscosity } \eta_{0}: & \eta_{0}=\lim _{\dot{\gamma} \rightarrow 0} \eta(\dot{\gamma}) . \\
\text { Infinite shear viscosity } \eta_{\infty}: & \eta_{\infty}=\lim _{\dot{\gamma} \rightarrow \infty} \eta(\dot{\gamma}) .
\end{aligned}
$$

Fig. 28(a) illustrates the fitting quality of the results obtained by the two most popular models (Cross [70] and Carreau [71]). The cross model for viscosity is

$$
\begin{gathered}
\frac{\eta(\dot{\gamma})-\eta_{\infty}}{\eta_{0}-\eta_{\infty}}=\frac{1}{1+\left(\frac{\dot{\gamma}}{\dot{\gamma}_{c}}\right)^{p}}, \\
\text { simplified form : } \frac{\eta(\dot{\gamma})}{\eta_{0}}=\frac{1}{1+\left(\frac{\dot{\gamma}}{\dot{\gamma}_{c}}\right)^{p}} .
\end{gathered}
$$

$\dot{\gamma}_{c}$ is the critical shear rate for the onset of a shear thinning response. As the shear rate approaches the critical shear rate, shear induced ordering becomes more prominent than the thermal randomizing in the liquid sample. It results in a steady decrease in apparent viscosity with an increasing shear rate (Region II). After attaining the maximum possible shear-induced ordering in the liquid sample, the apparent viscosity becomes independent of the applied shear rate (Region III). High shear Newtonian behavior, Region III, is not really observed for any $5 \mathrm{CB}$-TTAB samples within the investigated range of shear rates (Fig. 27(a)). $p$ is a parameter related to the slope of Region II. For the simplified form (Eq. 20 ), it is assumed that $\eta_{\infty}$ is very small in comparison to $\eta_{0}$.

The Carreau model for viscosity is

$$
\begin{gathered}
\frac{\eta(\dot{\gamma})-\eta_{\infty}}{\eta_{0}-\eta_{\infty}}=\frac{1}{\left(1+\left(\frac{\dot{\gamma}}{\dot{\gamma}_{c}}\right)^{2}\right)^{p}}, \\
\text { simplified form : } \frac{\eta(\dot{\gamma})}{\eta_{0}}=\frac{1}{\left(1+\left(\frac{\dot{\gamma}}{\dot{\gamma}_{c}}\right)^{2}\right)^{p}} .
\end{gathered}
$$

Simplified forms of both models are used for fitting to the data (Fig. 28(a)). It is quite evident from Fig. 28(a) that the Carreau model fits the experimental data better than the 


\begin{tabular}{|l|l|l|c|}
\hline $\begin{array}{c}5 \text { CB concentration } \\
(\text { wt\% })\end{array}$ & $\begin{array}{l}\text { Zero shear viscosity } \\
(\text { Pa.s })\end{array}$ & $\begin{array}{l}\text { Critical shear rate } \\
\left(s^{-1}\right)\end{array}$ & $\mathrm{p}$ \\
\hline 0 & 0.01329 & - & - \\
\hline 0.5 & 0.04764 & 4892.81892 & 0.14385 \\
\hline 1 & 0.16851 & 1585.46587 & 0.29969 \\
\hline 1.5 & 0.66327 & 561.53676 & 0.46352 \\
\hline 2 & 1.75505 & 234.32463 & 0.60475 \\
\hline 2.5 & 3.01969 & 131.15627 & 0.67494 \\
\hline 3 & 3.49454 & 124.5805 & 0.66595 \\
\hline 3.5 & 3.08155 & 166.47605 & 0.5967 \\
\hline 4 & 2.95213 & 181.13027 & 0.5883 \\
\hline 4.5 & 3.05315 & 178.04231 & 0.58039 \\
\hline 5 & 3.05136 & 172.09482 & 0.57035 \\
\hline
\end{tabular}

Table 4: Carreau model fitting parameters for 5CB in TTAB 25 wt\% solutions. All the three parameters in the simplified Carreau model are listed as a function of $5 \mathrm{CB}$ concentration.

Cross model. Values of the parameters $\left(\eta_{0}, \dot{\gamma}_{c}\right.$ and $\left.\mathrm{p}\right)$ obtained from the Carreau model are shown in Table 4.

As shown in Fig. 28(b) the zero-shear Newtonian viscosity value, $\eta_{0}$, significantly increases with $5 \mathrm{CB}$ concentration up to $3 \mathrm{wt} \%$. After that there is a slight decrease in the $\eta_{0}$ value until the solution phase $\left(L_{1}\right)$ ends. However, the $\eta_{0}$ value does stay approximately constant within the explored range of the droplet emulsion phase as shown in Fig. 28(b) (3.5 $\mathrm{wt} \%-5 \mathrm{wt} \% 5 \mathrm{CB}$ ). The presence of a maximum in $\eta_{0}$ values (at $3 \mathrm{wt} \% 5 \mathrm{CB}$ ) does indicate the presence of two different ways that micells are structurally changed upon addition of $5 \mathrm{CB}$ on either side of the maximum.

As mentioned earlier, systematic neutron scattering investigations revealed that pure
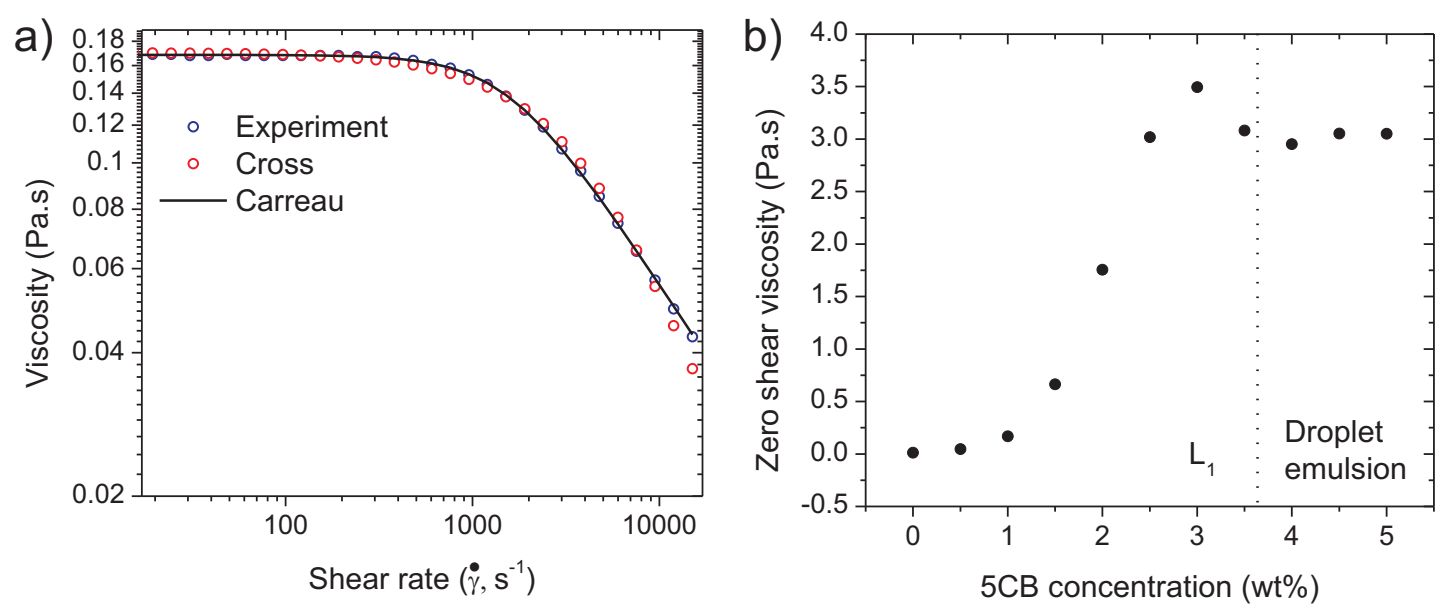

Figure 28: Comparing models for a steady shear flow curve of 5CB in TTAB 25 wt\% solution. (a) Cross and Carreau models are fitted for steady shear flow curve of 1 wt\% 5CB in TTAB 25 wt\% solution. The Carreau model evidently provides better fitting for this $5 \mathrm{CB}$ concentration. At higher $5 \mathrm{CB}$ concentrations, both models fail to predict values at high shear rates. (b) Zero shear viscosity values of 5CB-TTAB $25 \mathrm{wt} \%$ systems are obtained from the Carreau model. Rheological experiments for droplet emulsions are performed after homogenizing the emulsions. 
TTAB micelles are already prolate ellipsoidal in shape at $25 \mathrm{mM}(0.83 \mathrm{wt} \%)$ concentration (average axial ratio $\sim 1.43$ ) [44]. While the minor axes (a and b) of the ellipsoids are almost independent of TTAB concentration, the half-major axis c increases from $28.9 \AA$ at $25 \mathrm{mM}$ to $57.2 \AA$ at $673 \mathrm{mM}$ (18.5 wt\%). Extrapolating these values to $25 \mathrm{wt} \%$ gives $23.7 \AA$ and $72.9 \AA$ as half-minor axes and half-major axis values of TTAB micelles respectively. This gives the average axial ratio of the micelles at $25 \mathrm{wt} \%$ as $\sim 3.1$. Zero shear viscosity of the pure TTAB $25 \mathrm{wt} \%$ solution is $13.3 \mathrm{mPas}$. As we increase the concentration of $5 \mathrm{CB}$ in TTAB $25 \mathrm{wt} \%$ solutions, we see a dramatic increase in viscosity (3.5 Pas for $3 \mathrm{wt} \% 5 \mathrm{CB}$ in TTAB $25 \mathrm{wt} \%$ solution). The addition of $3 \mathrm{wt} \% 5 \mathrm{CB}$ in TTAB $25 \mathrm{wt} \%$ solution increases viscosity by a factor of $\sim 250$. This clearly suggests that the contour length of the micelles must be increased as we add $5 \mathrm{CB}$ to TTAB solution. Even a slight increase in the contour length should be enough to explain the observations as TTAB $25 \mathrm{wt} \%$ solution is already a concentrated charged micellar solution. As mentioned earlier, it is interesting to note that the addition of more $5 \mathrm{CB}$ to $3 \mathrm{wt} \% 5 \mathrm{CB}$-TTAB $25 \mathrm{wt} \%$ solution has a negative impact on the viscosity of the $5 \mathrm{CB}-\mathrm{TTAB}$ system. These observations for the 5CB-TTAB system fit perfectly into the observations made for the solubilization of polar molecules in the micellar solutions [66, 67, 72-74].

Non-polar molecules such as saturated aliphatic and alicyclic compounds solubilize in the inner core region of the micelles (between the ends of surfactant tails, Fig. 26(b)). The situation with polar or polarizable solubilizates is slightly complicated as they have multiple possible loci of solubilization. The extent of the solubilization of polar molecules depends heavily on the locus of the solubilization. A two-state model is proposed for polar or polarizable solubilizates [75]. Solubilizates are distributed between an adsorbed state at the micellar-water interface and a dissolved state in the micellar core. A solubilizate with high polarity favors the adsorbed state but increased concentration of the solubilizate produces a redistribution favoring the dissolved state. This two-state model can explain the existence of a maximum in zero shear viscosity vs a $5 \mathrm{CB}$ concentration plot (Fig. $28(\mathrm{~b})$ ). $5 \mathrm{CB}$ is a polarizable molecule owing to its $\pi$-electron cloud of the aromatic nucleus. The interaction between the $\pi$-electron cloud of $5 \mathrm{CB}$ and the positively charged quarternary ammonium group of the surfactant at the micelle-water interface results in the preferential dissolution of $5 \mathrm{CB}$ molecules at or near the micelle-water interface (adsorption state). Adsorption of $5 \mathrm{CB}$ molecules at or near the micelle-water interface reduces the electrostatic repulsion between head groups of the surfactants and thereby promotes the uniaxial growth of the prolate ellipsoidal TTAB micelles along its major axis. This increase in the contour length of the TTAB micelles explains the dramatic increase in viscosity of the 5CB-TTAB system until $3 \mathrm{wt} \% 5 \mathrm{CB}$ concentration. When the outer region of the micelle is saturated with $5 \mathrm{CB}$, further solubilization takes place inside the micellar core. This results in a transition from prolate ellipsoidal to slightly globular TTAB micelles, which explains a slight decrease in the viscosity as we add more $5 \mathrm{CB}$ to $3 \mathrm{wt} \% 5 \mathrm{CB}$ in the TTAB $25 \mathrm{wt} \%$ system (a reduction in the contour length of the micelle). Neutron scattering data is required to back up this structural transformation speculation based on the rheology data. Neutron scattering experiments also provide the values of the degree of the ionization, $\alpha$, of the micelles. It is extremely important to know the value of the degree of ionization of the micelles as it can have a profound effect on the values of the major axis of micelles and viscosity. More discussion about the effect of $\alpha$ on the viscosity is provided in Section 3.1.6.4.

Fig. 29(a) shows the relationship between shear stress and applied shear rates for 5CBTTAB $25 \mathrm{wt} \%$ systems. Shear stress plateaus exist in certain regions of applied shear rates for solutions with higher concentrations of $5 \mathrm{CB}$. The critical shear rates at which stress plateaus start, decrease with increasing $5 \mathrm{CB}$ concentration. The general explanation for 

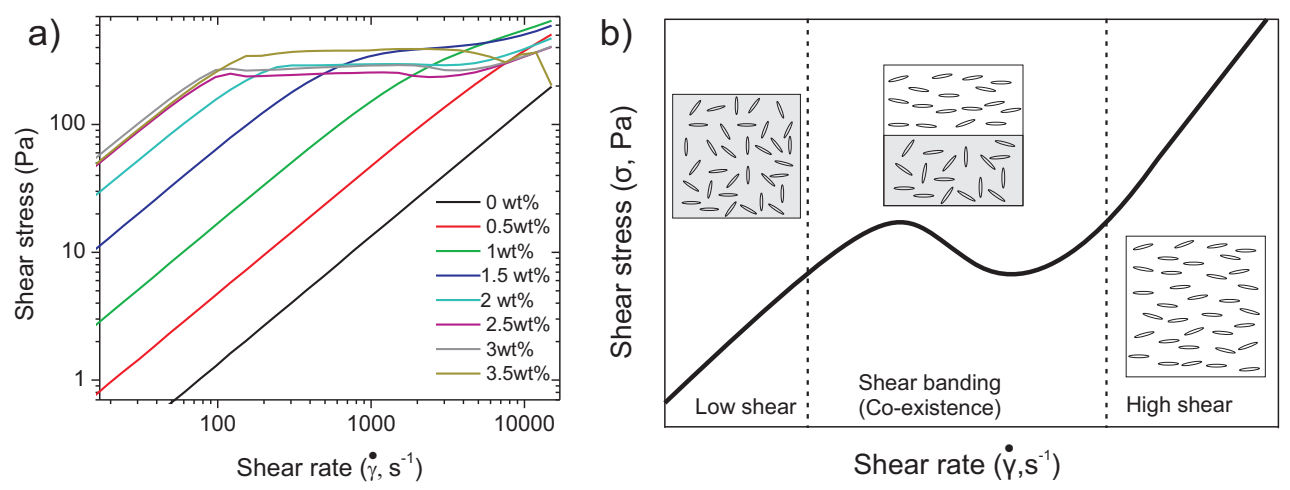

Figure 29: Shear banding phenomenon in 5CB-TTAB 25 wt\% systems. (a) Shear stress as a function of the shear rates for different concentrations of $5 \mathrm{CB}$ in TTAB $25 \mathrm{wt} \%$ solutions. (b) Schematic flow curve of a material exhibiting shear banding. At a fixed stress, two significantly different shear rates may co-exist.

stress plateaus is the shear banding phenomenon (Fig. 29(b)). Shear banding has been observed in a variety of systems such as worm-like micellar solutions, colloidal crystals and lamellar surfactant systems [76-81]. Shear banding can be interpreted as a system undergoing Isotropic-Nematic phase transition partially where a disordered phase (Isotropic) coexists with an ordered phase (Nematic). More experimental studies such as flow birefringence and neutron scattering are necessary to understand this type of flow instability.

\subsubsection{2 nCB compounds in TTAB $25 \mathrm{wt} \%$ solutions: Effect of added sol- ubilizate chain length on steady shear flow properties}

The effect of the solubilizate's chain length on the viscosity of the micellar solutions is investigated by using homologous series of 4'-n-alkyl-4-cyanobiphenyls (nCB series where $\mathrm{n}=2$ to 12 ). The molar concentration of LC molecules in all TTAB $25 \mathrm{wt} \%$ solutions is kept constant at $72.9 \mathrm{mM}$. It is not quite possible to go beyond this concentration as higher homologues have very limited solubility in TTAB $25 \mathrm{wt} \%$ solutions. This limited solubility of the higher homologues in the $\mathrm{nCB}$ series is due to their longer terminal alkyl chain lengths. For micellar shapes which are not so asymmetrical, more solubilization sites are available at the micelle-water interface than at the hydrocarbon core of the micelle. Thus, the solubilizates that are solubilized close to the micelle-water interface should be solubilized more than the solubilizates whose locus of solubilization lies in the micellar core. As the terminal alkyl chain length increases in the $\mathrm{nCB}$ compounds, they preferentially solubilize more and more deeply into the palisade layers of the micelles (Fig. 26(c)). Thus, the longer the terminal alkyl chain length of the $\mathrm{nCB}$ compounds, the smaller the degree of the solubilization.

Fig. 30(a) shows the steady shear rate $(\dot{\gamma})$ vs the apparent viscosity curves for $72.9 \mathrm{mM}$ nCB compounds in TTAB 25 wt\% solutions. The 12CB-TTAB system shows Newtonian behavior over the entire range of investigated shear rates. All other nCB-TTAB systems show shear thinning behavior. It is quite interesting to note that the addition of $12 \mathrm{CB}$ molecules to the TTAB $25 \mathrm{wt} \%$ solution has almost no effect on the viscosity. Fig. 30(b) shows the effect of the chain lengths of the solubilizates on the zero shear viscosities of the TTAB solutions. The Carreau model (Eq. 22) is used to obtain zero shear viscosity values. At this chosen molar concentration of $72.9 \mathrm{mM}$, the addition of $3 \mathrm{CB}$ molecules produces the maximum zero shear viscosity value. The interaction of the solubilizate with water molecules at the micelle-water interface (case- I), the polar head group of the surfactant 
(case- II) and the surfactant hydrophobic tail (case- III) determines the micellar structure and the viscosity of the solution. Methylene groups are electron-donating in nature. Thus, an increase in the terminal alkyl chain length increases the interaction of the $\pi$-electron cloud of nCB molecules with polar head groups of the surfactant (case-II). This results in the promotion of uniaxial growth of the TTAB micelles along its major axis. Increasing the terminal alkyl chain length results in an increase of the van der Waals interaction between solubilizate and surfactant tail (case- III). Finally, increasing the terminal alkyl chain length weakens the solubilizate's interaction with water molecules at the micelle-water interface due to an increase in the hydrophobicity of the solubilizate (case- I). While case-II favors the adsorbed state for solubilizates at the micelle-water interface, cases I and III favor the dissolved state in the hydrocarbon core. As a result, case-II promotes the formation of asymmetrical micelles (increase in viscosity) and cases I \& III promote the formation of globular micelles (reduction in viscosity). The presence of the maximum in zero shear viscosity values at $3 \mathrm{CB}$ shows that the collective effect of cases I and III take over the effect of case- II as the terminal alkyl chain length of the solubilizate increases. The insignificant effect of the addition of $12 \mathrm{CB}$ molecules to the viscosity of the TTAB $25 \mathrm{wt} \%$ solution suggests that all the three effects cancel each other out.

The addition of two different cyanobiphenyls at the same time in a TTAB solution provides further insight into the interaction between solubilizates and micelles. Fig. 30(c) shows the steady shear rate vs viscosity curves for a few micellar solutions. The addition of $54 \mathrm{mM} 12 \mathrm{CB}$ to the TTAB $25 \mathrm{wt} \%$ solution does not change the viscosity. It is quite interesting to note that $54.5 \mathrm{mM} 5 \mathrm{CB}-54.5 \mathrm{mM} 12 \mathrm{CB}$ mixture in the TTAB $25 \mathrm{wt} \%$ solution shows the same flow behavior as $54 \mathrm{mM} 5 \mathrm{CB}$ in the TTAB $25 \mathrm{wt} \%$ solution. This indicates that $5 \mathrm{CB}$ and $12 \mathrm{CB}$ molecules solubilize in TTAB micelles independently. However, this experiment does not provide information about the possibility of finding both $5 \mathrm{CB}$ and $12 \mathrm{CB}$ molecules simultaneously in the same TTAB micelle.
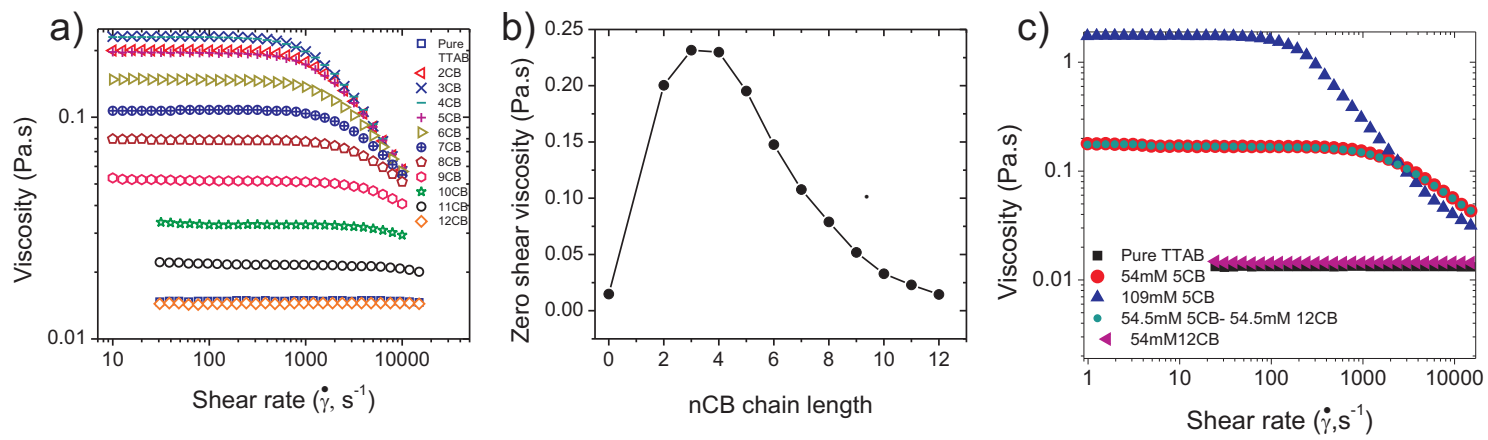

Figure 30: Effect of the molecular chain length of solubilizate on viscosity values. (a) Steady shear flow curves for several solubilizates from nCB series in TTAB $25 \mathrm{wt} \%$ solution at $23^{\circ} \mathrm{C}$. "Pure TTAB" in the legend represents pure TTAB 25 wt\% solution. The solubilizate's concentration is fixed at $72.9 \mathrm{mM}$. (b) Zero shear viscosity values for above steady shear flow curves using the simplified Carreau model. " 0 " value in the abscissa represents pure TTAB 25 wt\% solution. (c) The effect of the addition of two different solubilizates (5CB and $12 \mathrm{CB}$ ) simultaneously in a TTAB $25 \mathrm{wt} \%$ solution at $23^{\circ} \mathrm{C}$. Please note that $109 \mathrm{mM}$ $12 \mathrm{CB}$ is not completely soluble in TTAB $25 \mathrm{wt} \%$ solution. 


\subsubsection{5CB in TTAB $25 \mathrm{wt} \%$ solutions: Oscillatory shear measurements}

Oscillatory shear measurements were performed to understand the viscoelastic behavior of the $5 \mathrm{CB}$ in TTAB $25 \mathrm{wt} \%$ solutions. Fig. 31(a) shows the variation of the dynamic moduli (elastic or storage modulus $\left(\mathrm{G}^{\prime}\right)$ and viscous or loss modulus $\left(\mathrm{G}^{\prime \prime}\right)$ ) with oscillation frequency $(\omega)$ for different concentrations of $5 \mathrm{CB}$ in TTAB $25 \mathrm{wt} \%$ solution. The storage modulus values are not shown for $0.5 \mathrm{wt} \%$ of the $5 \mathrm{CB}-\mathrm{TTAB} 25 \mathrm{wt} \%$ solution. The storage modulus values of pure TTAB $25 \mathrm{wt} \%$ and $0.5 \mathrm{wt} \% 5 \mathrm{CB}-\mathrm{TTAB} 25 \mathrm{wt} \%$ solutions are well below the accuracy limit of the rheometer. The storage modulus values increase with increasing 5CB concentration in the TTAB micellar solution. However for all investigated $5 \mathrm{CB}$ concentrations, storage modulus values are always less than loss modulus values at all applied frequencies $\left(\mathrm{G}^{\prime \prime}>\mathrm{G}^{\prime}\right)$. Thus it is clear that all investigated 5CB-TTAB solutions show only a liquid-like behavior at all applied frequencies. This type of exclusive liquid-like behavior is generally shown by dilute random coil polymer solutions and dilute suspensions of the rod-like particles [82]. Fig. 31(b) shows the typical behavior of the dynamic moduli for various systems. Worm-like micellar solutions always show viscoelastic behavior $[67,69]$. Thus, it is clear that the micelles in 5CB-TTAB $25 \mathrm{wt} \%$ systems are not worm-like.
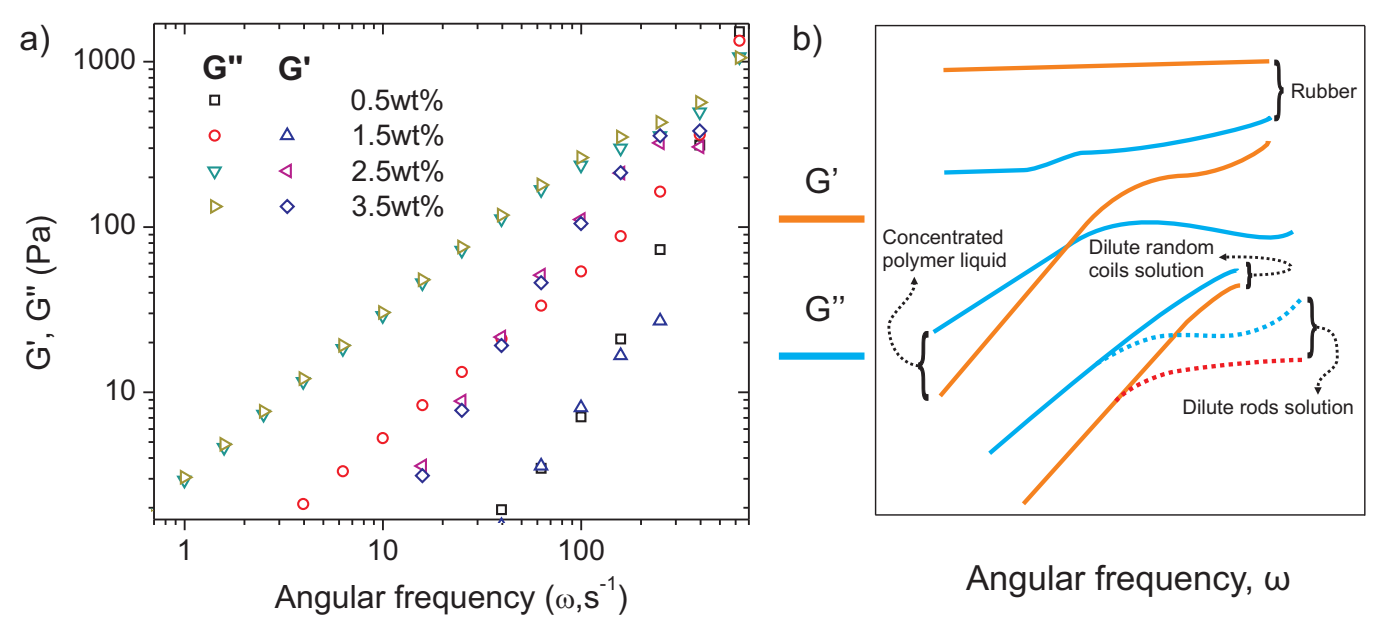

Figure 31: Oscillatory shear flow curves of 5CB in TTAB 25 wt\% solutions. (a) Dynamic shear moduli (elastic and viscous moduli) for different $5 \mathrm{CB}$ concentrations as a function of angular frequency. (b) Typical oscillatory shear behavior of various types of solutions are presented. The positions of each system are arbitrarily chosen in the plot.

\subsubsection{MBBA in TTAB 25 wt\% solutions: Steady shear flow curves}

As mentioned in the earlier sections, MBBA $(5.6 \mathrm{mg} / \mathrm{L})$ is eighty times more soluble than $5 \mathrm{CB}(0.07 \mathrm{mg} / \mathrm{L})$ in pure water at $25^{\circ} \mathrm{C}$. Because of this significant difference in the interaction with water, it is easy to expect that the phase behavior of the MBBA may also be significantly different from $5 \mathrm{CB}$ in TTAB $25 \mathrm{wt} \%$ solutions (see Section 3.1.1). It is interesting to note that the addition of MBBA in TTAB $25 \mathrm{wt} \%$ produces a lyotropic lamellar phase whereas TTAB on its own cannot form lamellar phases at all with water below $80^{\circ} \mathrm{C}$ [43]. The locus of solubilization of MBBA in a TTAB micelle should be much closer to the micelle-water interface (Fig. 26(a)) than in the case of 5CB due to its greater hydrophilic nature. Thus, space available for solubilization is greater for MBBA than for $5 \mathrm{CB}$. This may explain the much higher saturation limit for MBBA. 
$L_{1}$ Phase: $\quad$ Fig. 32(a) shows the steady shear rate $(\dot{\gamma})$ vs viscosity $(\eta)$ curves of MBBA in TTAB $25 \mathrm{wt} \%$ solutions at different MBBA concentrations within the solution phase region. Just as in the case of the $5 \mathrm{CB}-\mathrm{TTAB} 25 \mathrm{wt} \%$ solution phases, all the investigated MBBA-TTAB 25 wt\% samples show Newtonian behavior at low shear rates and shear thinning behavior at high shear rates. The Carreau model (Eq. 22) is used to obtain zero shear viscosity values $\left(\eta_{0}\right)$. But the Carreau model grossly overestimates the values of critical shear rates $\left(\dot{\gamma}_{c}\right)$ for MBBA concentrations above $4 \mathrm{wt} \%$ (in these cases, the Cross model (Eq. $20)$ is used to obtain the $\dot{\gamma}_{c}$ values). The changes in the $\eta_{0}$ values with increasing MBBA concentration are shown in Fig. 32(b). $\eta_{0}$ values of the 5CB-TTAB 25 wt\% systems are also added to Fig. 32(b) for comparison. Overlapping values of $\eta_{0}$ for both $5 \mathrm{CB}$ and MBBA systems (for concentrations below $3 \mathrm{wt} \%$ ) suggest that similar solulibizate-water-TTAB micelle interactions are in action in both cases. But arguments provided for the rheological behavior of the 5CB-TTAB $25 \mathrm{wt} \%$ system cannot explain the complete rheological behavior of MBBA in TTAB $25 \mathrm{wt} \%$ micellar solutions, i.e., the significant decrease in the $\eta_{0}$ values for MBBA concentration above $3 \mathrm{wt} \%$ and the existence of a lamellar phase. The significant decrease in the zero shear viscosity values above $3 \mathrm{wt} \% \mathrm{MBBA}$ concentration is likely due to the counter-ion condensation on the TTAB micelles. Counter-ion condensation is a general phenomenon for charged interfaces. Osawa and Manning showed that when the charge density on an infinitely long cylinder is increased beyond a certain critical value, counterions condensate onto the cylinder in order to reduce the effective charge density $[83,84]$. Similarly, in the case of ionic micelles the degree of counter-ion binding to the micelle (1- $\alpha)$ is very much related to the surface area per head group $\left(a_{m}\right)$ in the micelle $[65,85]$. Counterion binding increases as the $a_{m}$ value is decreased (i.e., degree of ionization $\alpha$ decreases). The solubilization of MBBA in the TTAB micelle decreases the $a_{m}$ value and in turn increases the degree of the counter-ion binding. Reduction in the $\alpha$ value has a profound effect on the rheology of the ionic micellar solutions (i.e., charged colloidal suspension) [86] and induces the growth of the micelles. The growth of micelles further enhances the counterion condensation due to the further reduction in the $a_{m}$ value [65]. This self-amplifying process makes the micelles grow in such a way that lamellar bilayers start to form in the micellar solution above a $6.4 \mathrm{wt} \%$ MBBA concentration in TTAB $25 \mathrm{wt} \%$ at $23^{\circ} \mathrm{C}$. Optical microscopy observations show that birefringence continuously increases upon the further
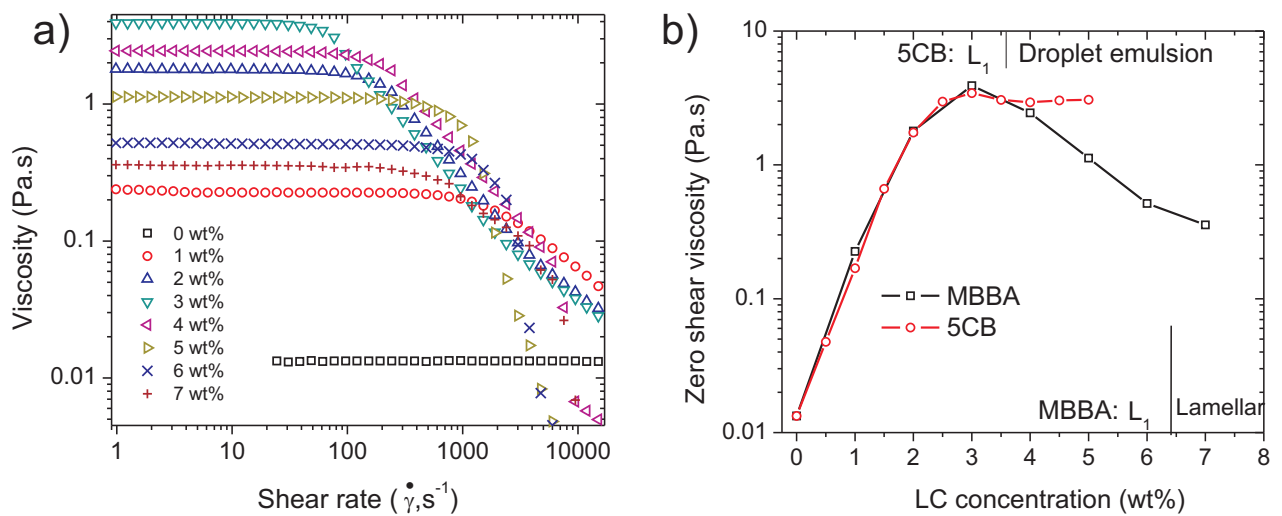

Figure 32: Steady shear flow curves of $L_{1}$ phase $M B B A-T T A B 25$ wt $\%$ solutions at $23^{\circ} \mathrm{C}$. (a) Steady shear flow curves are plotted for several MBBA concentrations. Even though 7 wt\% MBBA produces a birefringent phase in a TTAB $25 \mathrm{wt} \%$ solution, its flow behavior is still similar to $L_{1}$ phase solutions. (b) Zero shear viscosity values of above flow curves are obtained from the Carreau model. Zero shear viscosity values of 5CB-TTAB $25 \mathrm{wt} \%$ systems are also included. 
addition of MBBA until it reaches 14 wt\% (Fig. 14). As mentioned before, it is not yet clear whether this birefringent phase $\left(6.4 \mathrm{wt} \%-14 \mathrm{wt} \%\right.$ at $\left.23^{\circ} \mathrm{C}\right)$ is a single $L_{\alpha}$ phase or a mixture of $L_{1}$ and $L_{\alpha}$ phases.

The viscosity of ionic micellar solution is significantly greater than the viscosity of nonionic micellar solution with the exact same size and shape of the micelles [86]. Due to the electrical double layers (EDL) surrounding the ionic micelles, the effective size of the micelle increases. In concentrated micellar solutions like TTAB $25 \mathrm{wt} \%$, the overlapping of the EDL of micelles produces strong repulsive forces that control the phase behavior and rheology. Thus, the viscosity of the ionic micellar solutions depends on the effective charge on the micelles as well as on their size and shape. The effective charge on the micelles decreases via counter-ion condensation in such a way that the effect of the change in the micellar shape and size on viscosity values becomes increasingly insignificant for high MBBA concentrations (> $3 \mathrm{wt} \%$ ) in the $L_{1}$ phase.

Birefringent phase: Several studies are conducted to investigate the rheological behavior of the lyotropic and thermotropic liquid crystalline phases [87-91]. LC phases always show a complex flow behavior as the mechanical deformations induce several structural changes. Generally bulk LC samples are not homogeneously aligned and contain several types of defects. In the current birefringent systems, an oily streak structure or a polydomain mosaic texture is observed (Fig. 15(b-c)). Fig. 33(a) shows the flow properties of the lyotropic liquid crystalline TTAB solutions as a function of MBBA concentration ( $8 \mathrm{wt} \%$ - $14 \mathrm{wt} \%$ ). Fig. 33(b) shows a representative plot of the flow behavior of MBBA-TTAB 25 wt\% samples with all observed shear-induced microstructural transformations.

$7 \mathrm{wt} \%$ MBBA in a TTAB $25 \mathrm{wt} \%$ solution still shows the typical behavior of a shear thinning micellar solution (Fig. 32(a)). However from 8 wt\% MBBA concentration onwards complex flow behaviors are observed. As shown in Fig. 33(b), five different regimes can be observed. At very low shear rates (Region I), a disordered birefringent sample shows a shear thinning behavior. In this regime, flow is maintained by the sliding of the bilayers upon each other. A power-law decrease in the apparent viscosity is a result of the alignment of the bilayer structures or oily streak structures along the flow direction. This shear-induced ordering results in less structural resistance to the flow. A Newtonian regime (Region II)
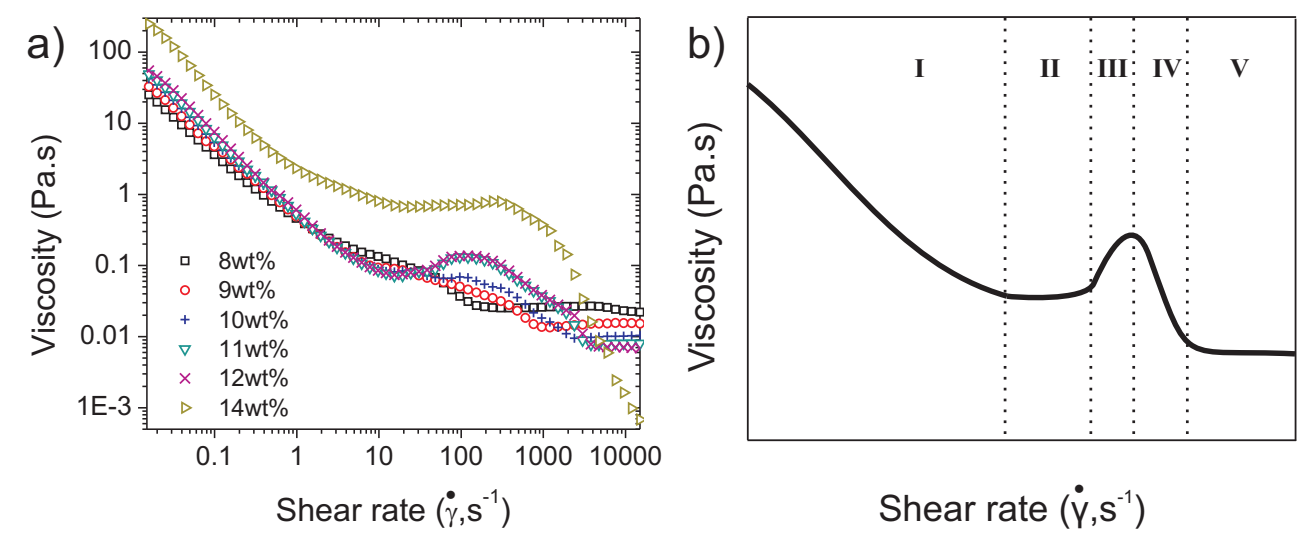

Figure 33: Steady shear flow curves of lamellar phase MBBA-TTAB 25 wt\% solutions at $23^{\circ} \mathrm{C}$. (a) Steady shear flow curves are plotted for MBBA concentrations between $8 \mathrm{wt} \%$ and $14 \mathrm{wt} \%$ (b) The generalized flow behavior of lamellar phase solutions is presented. Five different regions are observed. Region I: first shear thinning, Region II: first Newtonian plateau, Region III: shear thickening, Region IV: second shear thinning and Region V: second Newtonian plateau. 
follows with a further increase in the shear rate. The Newtonian regime expands with increasing MBBA concentration. A shear thickening regime (Region III) follows the Newtonian regime. Shear thickening is a result of the significant shear-induced microstructural change in the LC sample. Shear rates in this region are high enough to oscillate the bilayers in such a way that an undulation instability sets in. This undulation instability ultimately results in the formation of multi lamellar vesicles (MLVs) which disturb the flow. The increase in the number density of MLVs with increasing shear rate causes an increase in the apparent viscosity. After going through a local maximum in the viscosity, the LC sample experiences another shear thinning regime (Region IV). In this region, increasing the shear rate causes the alignment of the MLVs along the flow, as well as a reduction in the size of the MLVs. The last Newtonian regime (Region V) represents the complete rupture of MLVs and total alignment of the bilayers. Even though rheological responses of the LC samples are quite sufficient to understand the overall behavior, in situ optical observations and light scattering investigations are necessary to understand the shear induced microstructural transformations completely. Linear viscoelastic and transient flow experiments are also very much recommended.

\subsubsection{CB in TTAB 25 wt\% solution: Small-angle neutron scattering (SANS)}

Small angle neutron scattering is a powerful technique which is widely used to obtain structural information (size, shape and fractional charge on the micellar surface) of the micellar solutions [65-69]. A detailed structural analysis of pure TTAB micellar solutions using the SANS technique is already available [44]. TTAB micelles are almost spherical at or near critical micellar concentration (CMC). Increasing TTAB concentration favors the growth of the micelles along one of the axial directions (major axis) of the micelles. The growth of the micelles is restricted in the other two axial directions (minor axes) by the maximum chain length of the TTAB monomer in order to avoid the unfavorable penetration of the water molecules into the micelles. Thus at high concentrations, TTAB micelles are prolate ellipsoidal in shape. Our SANS results of the pure TTAB micellar solutions also point towards the prolate ellipsoidal shape of the TTAB micelles.

The addition of a solubilizate to the micellar solutions removes the restriction on the growth of the micelles along the minor axes if the locus of the solubilization of the solubilizate is at or near to the hydrophobic core. Thus, growth of the micelles significantly varies according to the interaction between the solubilizate, water and micelles. The rheological behavior of $5 \mathrm{CB}$ in TTAB $25 \mathrm{wt} \%$ micellar solutions does suggest changes in the TTAB micellar properties with increasing $5 \mathrm{CB}$ concentration. Preliminary neutron scattering experiments are done for 5CB in TTAB $25 \mathrm{wt} \%$ micellar solutions. Fig. 34(a) shows the SANS data for TTAB micellar solutions with varying $5 \mathrm{CB}$ concentrations $(0 \mathrm{wt} \%-3 \mathrm{wt} \%)$. Strong correlation peaks observed at all concentrations indicate the strong inter-micellar interactions. The peak value $\left(Q_{\max }\right)$ of the scattering intensity $(I(Q))$ curves shifts to the lower scattering vector $(Q)$ values as $5 \mathrm{CB}$ concentrations increase. $Q_{\max }$ is inversely related to the average distance between the micelles $(d)$. It is assumed that micelles are monodisperse with prolate ellipsoidal shapes with major axis, $a$, and minor axes value, $b$, for investigated $5 \mathrm{CB}$ concentrations.

$$
Q_{\max } \sim \frac{1}{d} \sim\left(\frac{N}{V}\right)^{\frac{1}{3}} \sim\left(\frac{\phi}{a b^{2}}\right)^{\frac{1}{3}}
$$




$$
\left(\frac{N}{V}\right)=\left(\frac{N}{N V_{m}+V_{E}} \cdot \frac{N V_{m}}{N V_{m}}\right)=\left(\frac{\phi}{V_{m}}\right) \sim\left(\frac{\phi}{a b^{2}}\right),
$$

where $N$ is the total number of micelles in the solution with total volume $V . \quad \phi$ is the volume fraction of the micelles in the solution. $N V_{m}$ and $V_{E}$ represent the volume occupied by all micelles in the solution and the remaining volume of the solution $(\sim$ solvent volume $)$ respectively. Assuming the volume fraction of the micelle and the minor axes value remain approximately constant during the addition of $5 \mathrm{CB}$,

$$
Q_{\max }=Q_{\max , 0}\left(\frac{a_{0}}{a}\right)^{\frac{1}{3}} .
$$

Here $Q_{\max , 0}$ and $a_{0}$ represent the peak value of the scattering curve and major axis value of the micelles of the pure TTAB $25 \mathrm{wt} \%$ solution respectively. Fig. 34(b) shows the changes in the values of $Q_{\max }$ and $a$ at various $5 \mathrm{CB}$ concentrations in TTAB $25 \mathrm{wt} \%$ solutions. The major axis value increases by approximately $28 \%$. Thus, an increase in viscosity by adding $5 \mathrm{CB}$ to TTAB $25 \mathrm{wt} \%$ solutions can be accounted for by the increase in contour length of the TTAB micelles. Unfortunately, SANS data is not available for $3.5 \mathrm{wt} \% 5 \mathrm{CB}$ concentration. Detailed quantitative SANS data analysis is yet to be done. SANS experiments for MBBA in TTAB 25 wt\% solutions are highly desired. It is also worthwhile finding out the locus of solubilization of solubilizates in the TTAB micelles for different solubilizate concentrations by using UV or NMR spectroscopy. Morphological changes in the micelles induced by the addition of a solublizate can further be confirmed by using the cryo-TEM technique $[69,74,76]$.
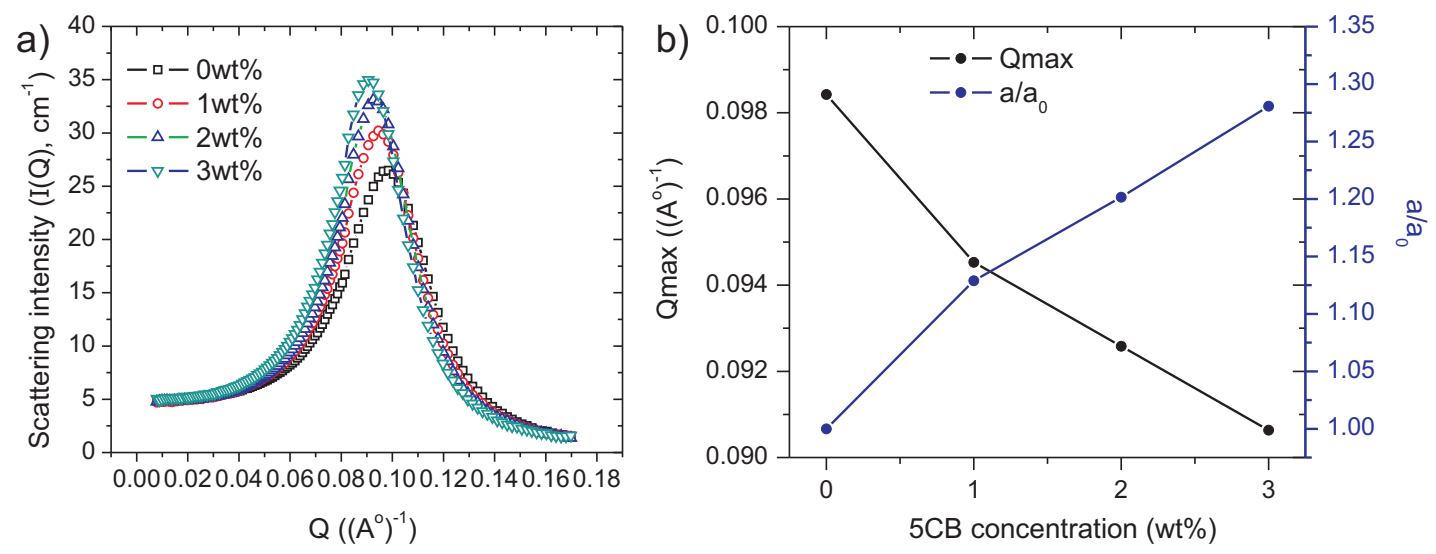

Figure 34: SANS spectra for 5CB-TTAB 25 wt\% systems with varying 5CB concentration. The right image shows the variation of peak positions in the SANS data (left image) and the major axis value as a function of $5 \mathrm{CB}$ concentration.

\subsubsection{CB droplets in aqueous ionic micellar solutions: Self- propelled motion}

Several types of interfacial instabilities may spontaneously arise when a ternary liquidliquid-amphiphile system (whose equilibrium state is a microemulsion) is initially brought into contact in a macroscopically phase separated state [92-95]. The strict thermodynamic meaning of the "spontaneity" is not a very useful one here as it refers only to the lowering of the free energy of the system without considering the kinetics of the process (i.e., whether the 
process occurs or not). In our case, the spontaneous process is characterized by negative free energy change as well as by a very low activation energy under the experimental conditions.

When a $5 \mathrm{CB}$ droplet is introduced into a pure water solution, there is no observable motion at both sides of the 5CB - water interface as shown in Fig. 35(a). But as mentioned before, the introduction of a $5 \mathrm{CB}$ droplet into an ionic micellar solution results in a spontaneous solubilization process. This solubilization process continues until all the active micelles in the aqueous medium are exhausted. Solubilization of 5CB in an ionic micellar solution has two side effects: (i) $5 \mathrm{CB}$ droplets propel themselves in the aqueous medium. This self-propelled motion of the $5 \mathrm{CB}$ droplets depends on the parameters such as surfactant concentration and temperature of the system. (ii) Along with self-propulsion of the 5CB droplets, strong convection is also observed in and around 5CB droplets. Convective flows
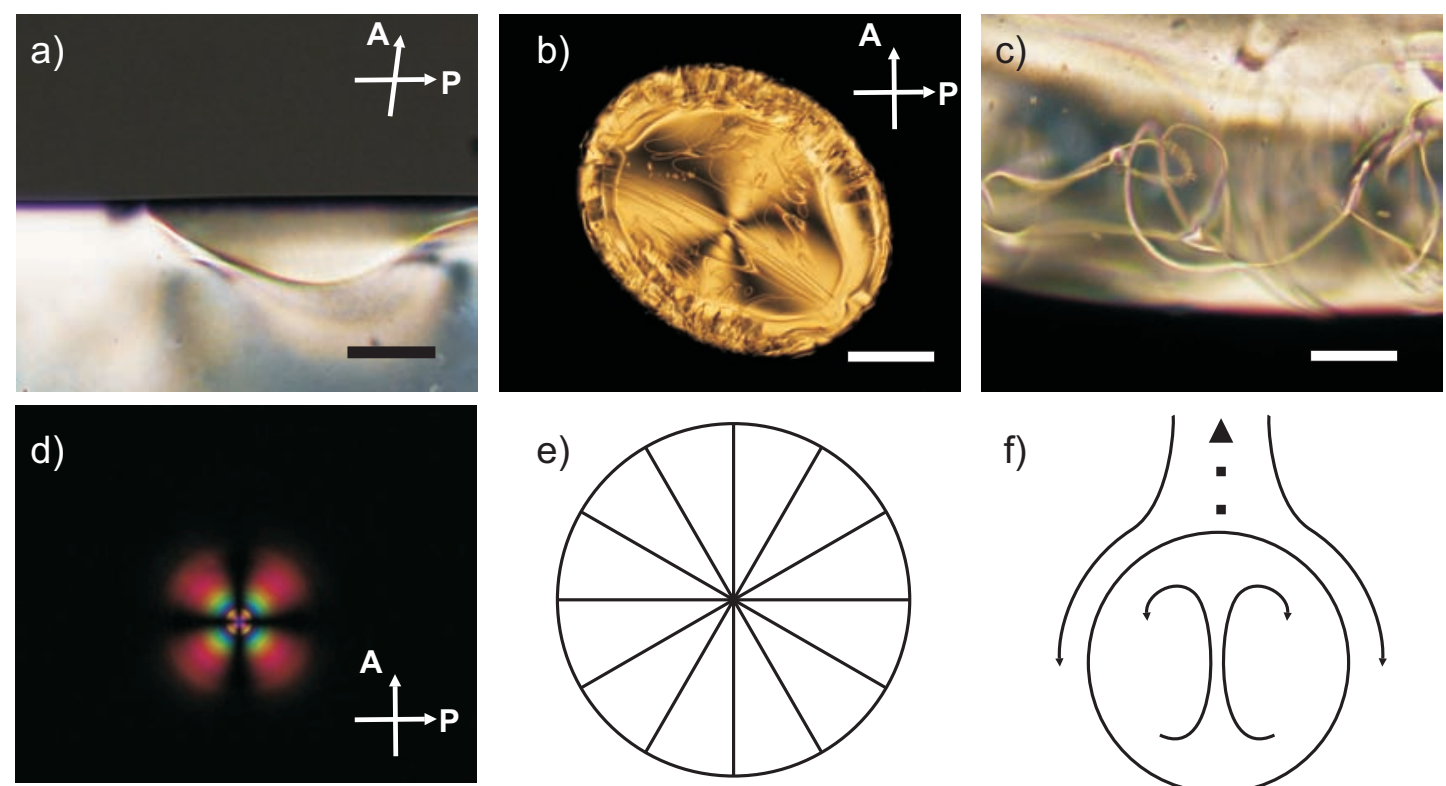

f)
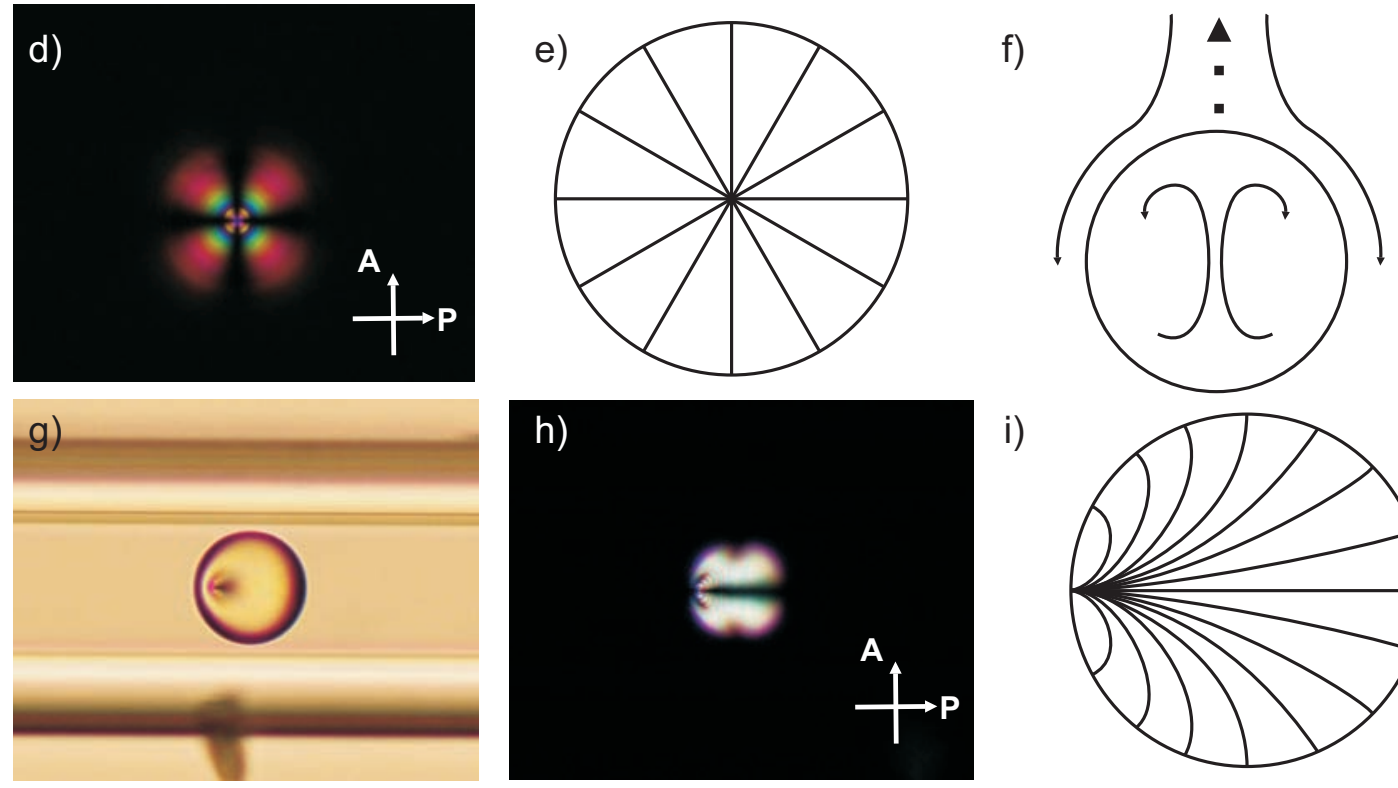

i)

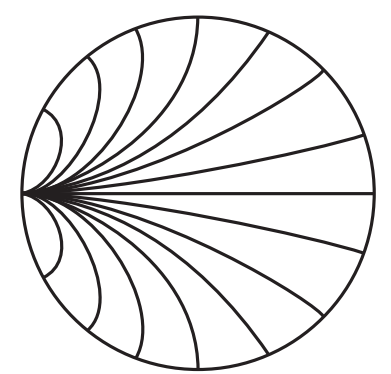

Figure 35: Nematic director profile in a self-propelled 5CB droplet. (a) The 5CB-pure water interface is absolutely still. Scale bar: $50 \mu \mathrm{m}$. (b) A pinned 5CB droplet in a TTAB 6 wt\% solution. Numerous disclination lines are generated due to the convective flow generated inside the $5 \mathrm{CB}$ droplet. Scale bar: $400 \mu \mathrm{m}$. (c) A magnified image of the same droplet's surface. Scale bar: $50 \mu \mathrm{m}$. (d) A crossed polarizer image of a $13.5 \mu \mathrm{m}$ radial nematic $8 \mathrm{CB}$ droplet in $\mathrm{CTAB} 5 \mathrm{mM}$ solution at $37^{\circ} \mathrm{C}$. (e) A schematic representation of the director orientation inside a radial nematic droplet. (f) A schematic representation of the flow fields inside and around a self-propelling $5 \mathrm{CB}$ droplet. The circle represents a $5 \mathrm{CB}$ droplet. Thin curved arrows inside and outside the circle represent the flow field inside and outside the droplet respectively. The thick dotted arrow represents the direction of the $5 \mathrm{CB}$ droplet motion. (g-h) A white light image and a crossed polarizer image of a self-propelling $5 \mathrm{CB}$ droplet in a square capillary whose inner side edge length is $50 \mu \mathrm{m}$. (i) A schematic representation of the director orientation structure inside a self-propelling nematic droplet. 
and self-propelled motion of the droplet do not need to happen simultaneously. Convection is still observed in the pinned droplets as shown in Fig. 35(b-c). Both convective flows and self-propelled motion become less pronounced with decreasing amphiphile concentration in the aqueous medium and stop altogether for concentrations below CMC of the amphiphile. Convective flows are generated due to the reorganization of the materials in the system. It is very clear from the optical observations that flows of materials exist in both directions at the $5 \mathrm{CB}$ - aqueous solution interface. The material transfer from the $5 \mathrm{CB}$ droplet into the micellar solution outweighs the material transfer from the aqueous solution into the $5 \mathrm{CB}$ droplet (i.e., the $5 \mathrm{CB}$ droplet radius always decreases over time). Material transfer pathways are yet very unclear and attempts are being made in our lab to determine these pathways. Material transfer across the interface induces interfacial tension gradients (Marangoni stresses) around the droplet which in turn drive the convective flows at the interface. Propulsion of the $5 \mathrm{CB}$ droplet depends upon the type and intensity of the convective flows in the aqueous solution (i.e., the hydrodynamic flow field around the droplet) [96].

Fig. 35(d-e) shows the nematic director field in a still 5CB droplet in a dilute ionic surfactant solution. Surfactants at the interface induce a strong homeotropic anchoring at the droplet surface which results in a point defect of strength +1 at the center of the droplet. Fig. 35(f-h) shows the effect of convective flow in the 5CB droplet on its director field. Convective flow within the self-propelled $5 \mathrm{CB}$ droplet drives the central point defect toward the surface of the droplet. Just as in this case, flow-induced director field transformations are also observed in bipolar nematic LC droplets [97]. However in the latter case, convective flows inside the bipolar LC droplets are not self-generated. It is interesting that the selfpropelled motion of $5 \mathrm{CB}$ droplets resembles the butterfly swimming stroke.

The behavior of self-propelled $5 \mathrm{CB}$ droplets in a quasi one-dimensional environment is investigated. Initially, a $5 \mathrm{CB}$ droplet dilute aqueous emulsion is created. A $5 \mathrm{~cm}$ long square capillary (the side length of the square cross-section is $50 \mu \mathrm{m}$ ) is dipped into the emulsion. Because of the capillary force, the emulsion spontaneously fills the capillary completely. Both ends of the capillary are finally closed with a two-minute epoxy. Experiments are made with $5 \mathrm{CB}$ droplets whose diameters are almost equal to the edge of the square capillary. If the final state of the created dilute aqueous emulsion is a microemulsion, then the $5 \mathrm{CB}$ droplets continue to self-propel themselves until they solubilize completely. Fig. 36(a) shows the displacement of a $5 \mathrm{CB}$ droplet in a capillary vs time curves for several aqueous micellar solutions. These optical observations show that $5 \mathrm{CB}$ droplets attain a constant velocity
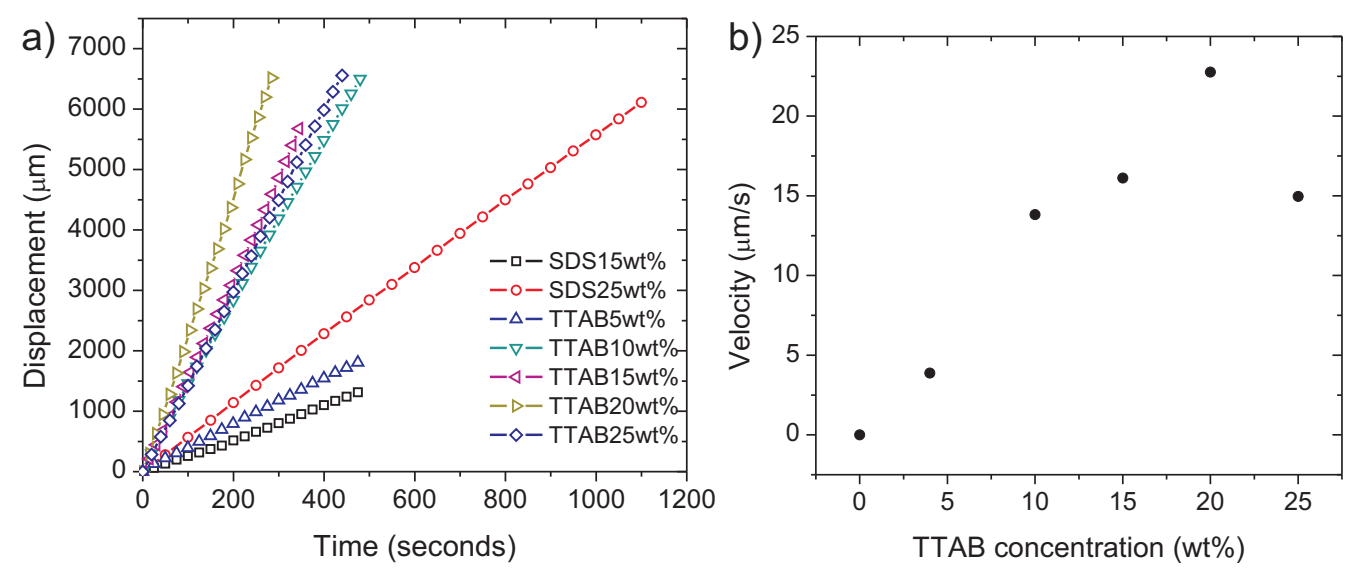

Figure 36: The equilibrium velocities of self-propelled $5 C B$ droplets vs surfactant concentration at $23^{\circ} \mathrm{C}$. (a) Displacement is plotted as a function of time, at different surfactant concentrations. (b) Velocity of a $5 \mathrm{CB}$ droplet as a function of TTAB concentration. 
almost instantly. At low TTAB concentrations, 5CB droplets move almost in straight lines. Fig. 36(b) shows that the maximum velocity of a $5 \mathrm{CB}$ droplet is attained with TTAB 20 wt $\%$ concentration. The apparent dip in the velocity value for TTAB $25 \mathrm{wt} \%$ concentration is due to the increased non-straight line motion of the $5 \mathrm{CB}$ droplet with increasing TTAB concentration (zigzag motion). The velocity values for TTAB and SDS solutions clearly suggest that they significantly depend on the efficiency and effectiveness of the surfactants.

Fig. 37 shows a time lapse representation of a head-on collision between two almost equal-sized self-propelled droplets in a square capillary. As long as the density of the active micelles around the droplets is not affected significantly by the continuous exhaust of the $5 \mathrm{CB}$ molecules from the droplets, the velocities of the droplets do not change even after the collision. Hydrodynamic flow fields around the droplets decide the possible proximity between the droplets during the collision process. As the concentration of the surfactant increases, droplets repel each other at increased distances and reverse their direction of the motion. Predictably, the nematic director fields in the moving droplets also reorient after
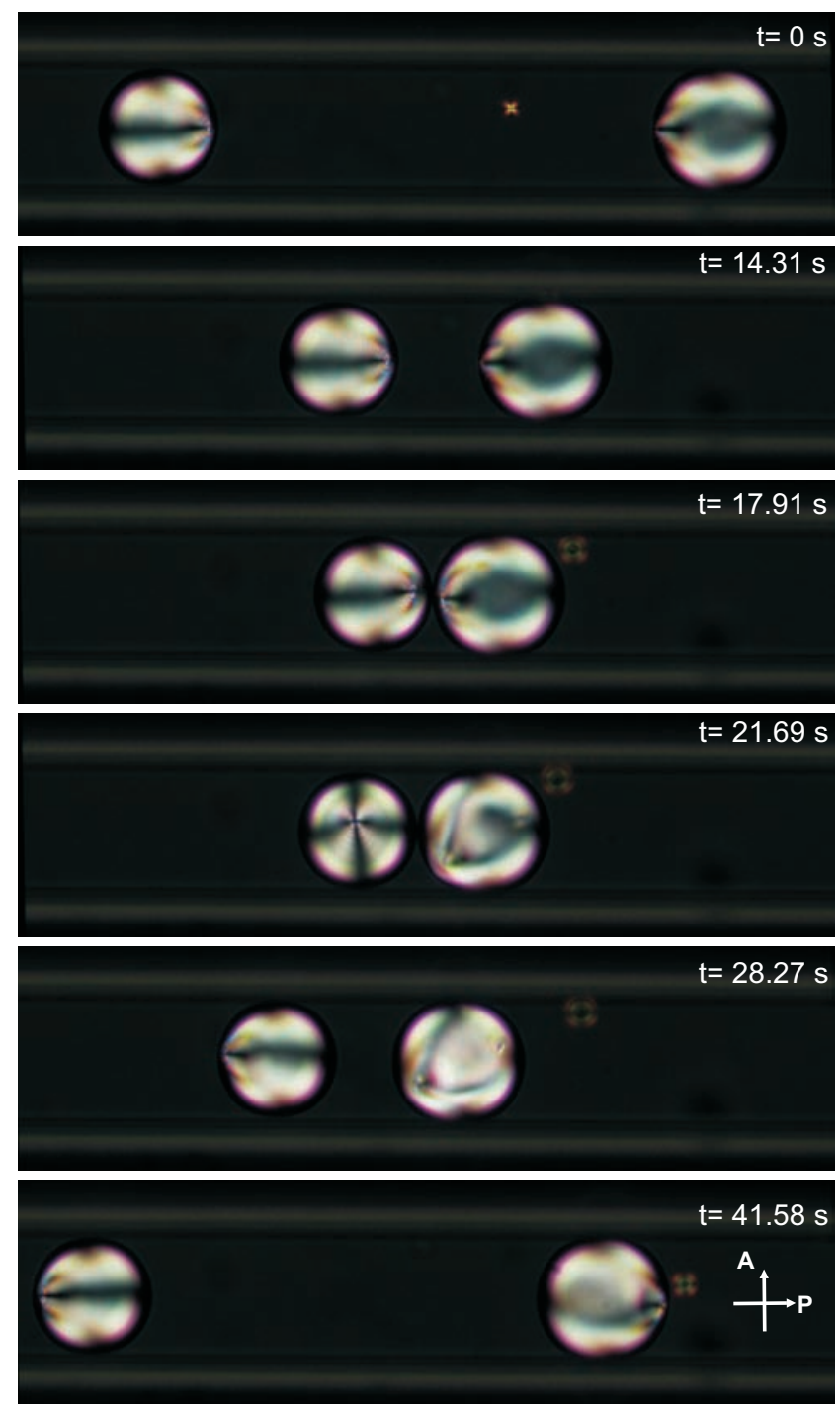

Figure 37: Head-on collision between two self-propelling 5CB droplets in a square capillary. The droplets' size is almost the same as the inner edge length of the square capillary (50 $\mu \mathrm{m})$. Any noticeable change in the equilibrium velocities of the droplets is not observed due to the reversal of their respective direction of motion. However, droplets take some time to reach their equilibrium velocities after the collision. 
the collision.

Self-propelled motion is not exclusive for nematic droplets. Motion also exists for droplets in isotropic, cholesteric and smectic states. The presence of the motion in the isotropic state suggests that a liquid crystalline structure in the droplet is not essential. However it was observed that surfactants induce a liquid crystalline thin film at the stationary isotropic oil-aqueous micellar solution interface $[98,99]$. These observations suggest that a liquid crystalline thin film may be present around a moving isotropic droplet but experimental evidence is necessary to back up this hypothesis. Self-propelled nematic and isotropic droplets have only translational motion but self-propelled cholesteric (chiral nematic) droplets have an extra rotational motion. The self-propelled motion of the smectic droplets is entirely different from the above mentioned LC phase droplets (nematic, isotropic and cholesteric) and preliminary observations are discussed in Section 3.2.6. Detailed investigation of the role of liquid crystallinity in the self-propelled motion is very much recommended.

\subsubsection{Nematic filaments}

When a $5 \mathrm{CB}$ droplet in the nematic state is introduced into a TTAB $25 \mathrm{wt} \%$ solution at $23^{\circ} \mathrm{C}$ whose final equilibrium state is the $L_{1}$ phase, strong convective flows are generated around the 5CB droplet. As discussed earlier, these convective flows produced by Marangoni stresses around the droplet may amplify the solubilization process as well as cause the selfpropelled motion of the droplet until it solubilizes completely in the TTAB solution. Another type of interfacial instability also comes into play if the effectiveness of the surfactant is increased considerably (effective surfactants reduce the interfacial tension values effectively). The interfacial tension value of the nematic LC and ionic micellar solution decreases with decreasing temperature [57]. The interfacial tension exhibits a maximum near the bulk LC oil Nematic - Isotropic $\left(T_{N I}\right)$ transition temperature. As reported in the earlier sections, $5 \mathrm{CB}$ droplets start to eject tiny $5 \mathrm{CB}$ daughter droplets at around $5^{\circ} \mathrm{C}$ in TTAB $25 \mathrm{wt} \%$ solution. This observation is only possible if the interfacial tension value of the $5 \mathrm{CB}$ droplet - aqueous micellar solution at $5^{\circ} \mathrm{C}$ is significantly less than the value at $23^{\circ} \mathrm{C}$. The dependence of the daughter droplet ejection in an MBBA-TTAB system on the effectiveness of the surfactant also supports this hypothesis (Fig. 21). Real-time dynamic interfacial tension measurements for 5CB-TTAB and MBBA-TTAB systems are very much needed. Similar spontaneous ejections of the daughter droplets due to ultra-low interfacial tension values are observed in some isotropic oil-water systems [100-102]. Low interfacial tension values are attributed to the existence of a weak lyotropic birefringent material at the interface of these systems. It is also noteworthy that LC parent droplets only eject the daughter droplets in TTAB x wt\% solution if the LC is able to form an equilibrium lamellar phase with TTAB $\mathrm{x}$ wt\% aqueous solution (at a given temperature and at least at any one particular weight ratio of LC to TTAB $\mathrm{x}$ wt\% aqueous solution). This suggests that spontaneous ejection of the daughter droplets from LC droplets in TTAB solutions during the solubilization may not be due to the nematic character of the droplet. Instead, it may be due to a possible lyotropic lamellar thin film around the LC droplets.

Single tail ionic surfactants such as TTAB are generally neither effective nor efficient in reducing the interfacial tension values due to the repulsion between head groups of the surfactant monomers at the interface. However, the addition of a co-surfactant can significantly reduce the interfacial tension [14]. When the interfacial tension between two immiscible liquids is ultralow or negative, the interface roughens and develops a variety of interfacial structures [93,94]. Unlike the earlier experiments of an LC droplet solubilization 

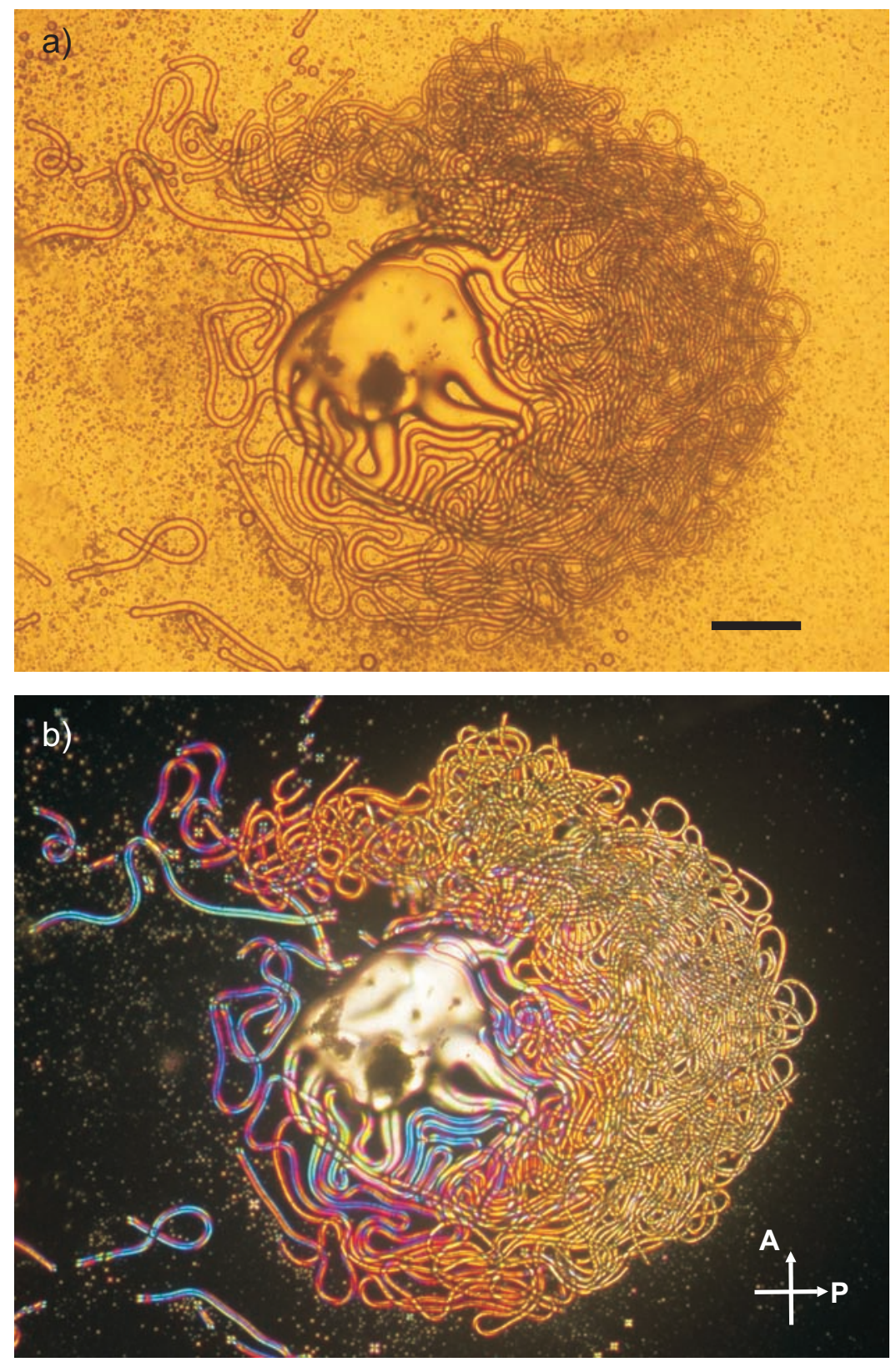

Figure 38: Interfacial instability in a large nematic $8 C B$ droplet. (a-b) A white light and a crossed polarizer image of a distorted nematic $8 \mathrm{CB}$ droplet in a CTAB $0.75 \mathrm{mM}$ solution. The $8 \mathrm{CB}$ droplet contains $2 \mathrm{wt} \%$ monoolein. Scale bar: $100 \mu \mathrm{m}$. Unlike small nematic droplets, large nematic droplets can host several defects. Low interfacial tension drives the nematic liquid crystal (surrounding the defects) to escape into a third dimension (i.e., into the aqueous medium) through the formation of numerous filaments.

in an aqueous micellar solution, this time a system is chosen in such a way that the micellar solubilization of LC molecules in the aqueous solution is negligible. As a result, it is possible to study the influence of the nematic character on the behavior of the LC-aqueous solution interface exclusively.

In recent years, there is an increased interest in exploiting the unique properties of the dispersion of liquid crystals droplets in immiscible isotropic mediums. Mostly these anisotropic dispersions are used for optical and sensing applications. Optical applications exploit the structural properties of the droplets of various liquid crystalline phases [103-106], sensing applications are based on the effect of external molecules on the anchoring properties 
of the LC (dispersed phase) - water (continuous phase) interface [107-109]. Apart from studies on anchoring, interfacial phenomena of the liquid crystal dispersions are largely unexplored [58,110-112]. This vacuum of knowledge propelled us to investigate the LC water interfacial behavior.

Surfactants are generally used for obtaining a strong homeotropic anchoring at an LC - water interface. But this also results in the reduction in the interfacial tension of the LC - water interface. When the interfacial tension decreases beyond a certain value, the LC-water interface expands spontaneously in order to reduce the total free energy of the system (i.e., by reducing the elastic distortions in the LC bulk). This effect is observed in a few LC droplet emulsions $[58,111,112]$. Toquer et al., [58] show that some speciallydesigned surfactants possessing a mesogenic moiety can transiently induce a shape instability in $5 \mathrm{CB}$ droplets through harsh temperature quenching. This shape instability results in the transformation from a spherical $5 \mathrm{CB}$ droplet to an elongated nematic filament. This report is unintentionally misleading as it demonstrates that harsh quenching and specially-designed amphitropic surfactants are required to induce this type of shape instability. Our findings suggest that none of the above conditions is necessary.

Experiments are done with $8 \mathrm{CB}$ in nematic state and aqueous CTAB solutions. CTAB is more efficient and effective than TTAB in reducing the interfacial tension values [14]. In order to nullify the micellar solubilization of $8 \mathrm{CB}$ in $\mathrm{CTAB}$ solutions, only concentrations below CMC are used $\left(\sim 1 \mathrm{mM}\right.$ at $\left.25^{\circ} \mathrm{C}\right)$. Even though CTAB is better than TTAB, it is still not good enough to create shape instabilities at the LC - aqueous solution interface. Thus, $8 \mathrm{CB}$ is doped with small amounts of the monoolein which acts as a co-surfactant. This combination of surfactants is sufficient enough to create an instability at the LC - aqueous solution interface as shown in Fig. 38. A stock emulsion of $8 \mathrm{CB}$ droplets is prepared in $0.06 \mathrm{mM}$ CTAB solution. Nematic filaments are produced when a small amount of this stock emulsion is brought into contact with $0.5 \mathrm{mM} \mathrm{CTAB}$ aqueous solution in a sandwich cell. The dimensions and the stability of nematic filaments unsurprisingly depend upon the surfactant concentrations and the temperature of the system.

\subsubsection{Reversible transformation: Isotropic droplet $\leftrightarrow$ nematic filament}

Interfacial tension between an $8 \mathrm{CB}$ droplet and the surrounding aqueous solution can be manipulated by changing the temperature of the system. Fig. 39(a) shows the reversible transformation of an isotropic droplet into a nematic filament. As the temperature is decreased, the isotropic $8 \mathrm{CB}$ droplet turns into a radial nematic droplet. A further decrease in the temperature changes the elastic constants of $8 \mathrm{CB}$ in such a way that a twisted radial configuration is preferred over a radial configuration [113]. The twisted radial droplet finally turns into a nematic filament upon a further decrease in temperature. The internal texture of the nematic filaments is a radial organization of molecules with an escaped core instead of an axial line defect [58]. It is important to note that this process is only reversible for a limited time. This is due to the continuous reorganization of the surfactants in the system. Monoolein slowly diffuses into the aqueous phase from the $8 \mathrm{CB}$ droplet over time. Due to this reason, monoolein concentration at the interface continuously decreases. As a result, the interfacial tension value increases over time and eventually this reversible shape instability vanishes. A more hydrophobic non-ionic surfactant than monolein should considerably rectify this problem. Fig. 40 also demonstrates the influence of the temperature on the surface area of a nematic filament. The surface area of a filament decreases with increasing temperature.

It is interesting to note that the point defect is necessarily present at its original position 

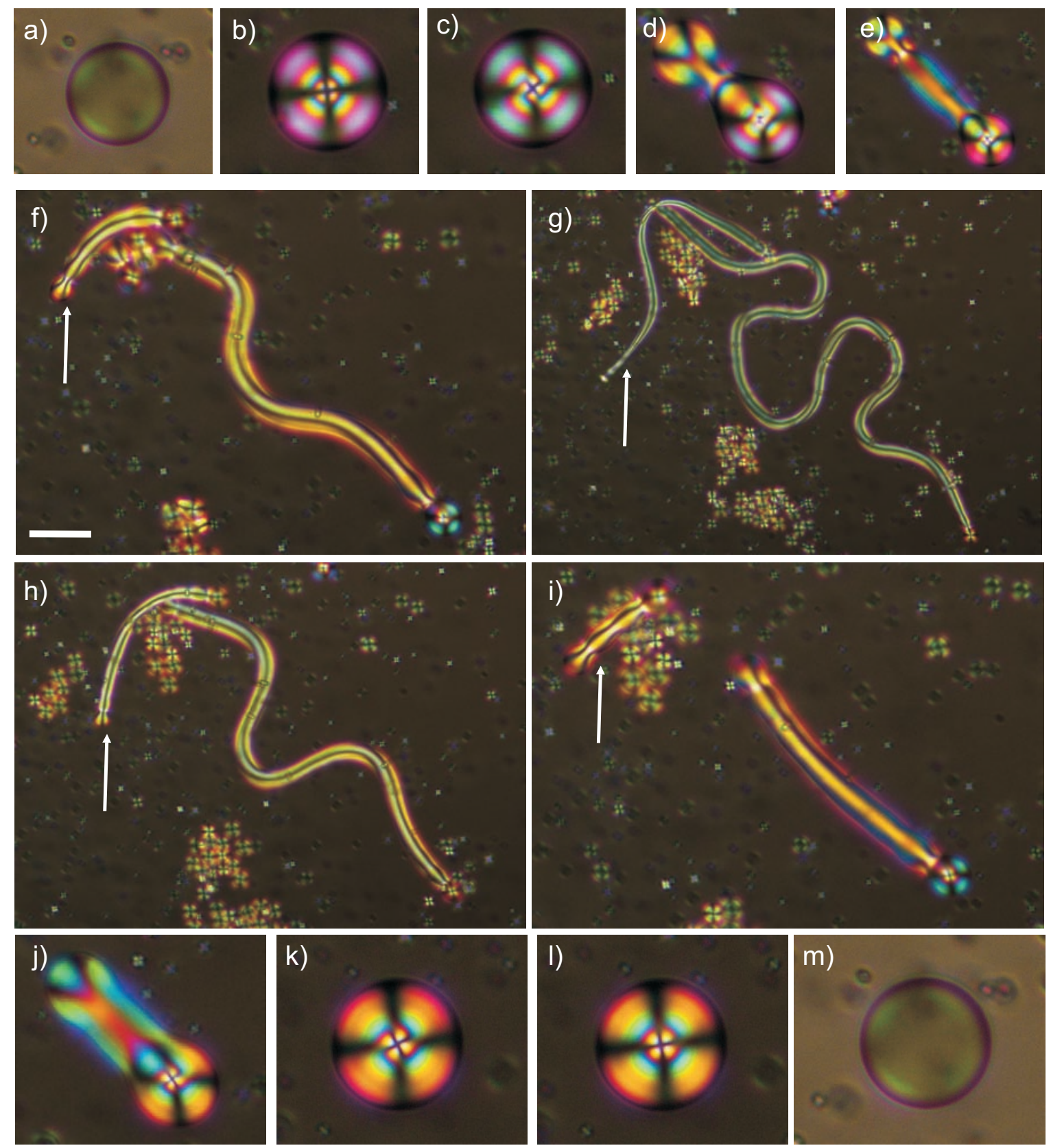

Figure 39: Reversible transformation between an isotropic droplet and a nematic filament. (a-g) A series of micrographs show the transformation from an isotropic droplet into a long thin nematic filament while cooling. Initial droplet size is $22 \mu \mathrm{m}$. (h-m) Exact opposite process while heating. During elongation, small nematic droplets are separated from the parent nematic filament at the growing end. The smaller filament observed in the figure (f-i) is further formed by one such separated droplet (indicated with arrow). Due to this division process, the droplet size in the figure $\mathrm{m}$ is $18 \mu \mathrm{m}$. Scale bar in image f: $14 \mu \mathrm{m}$. 

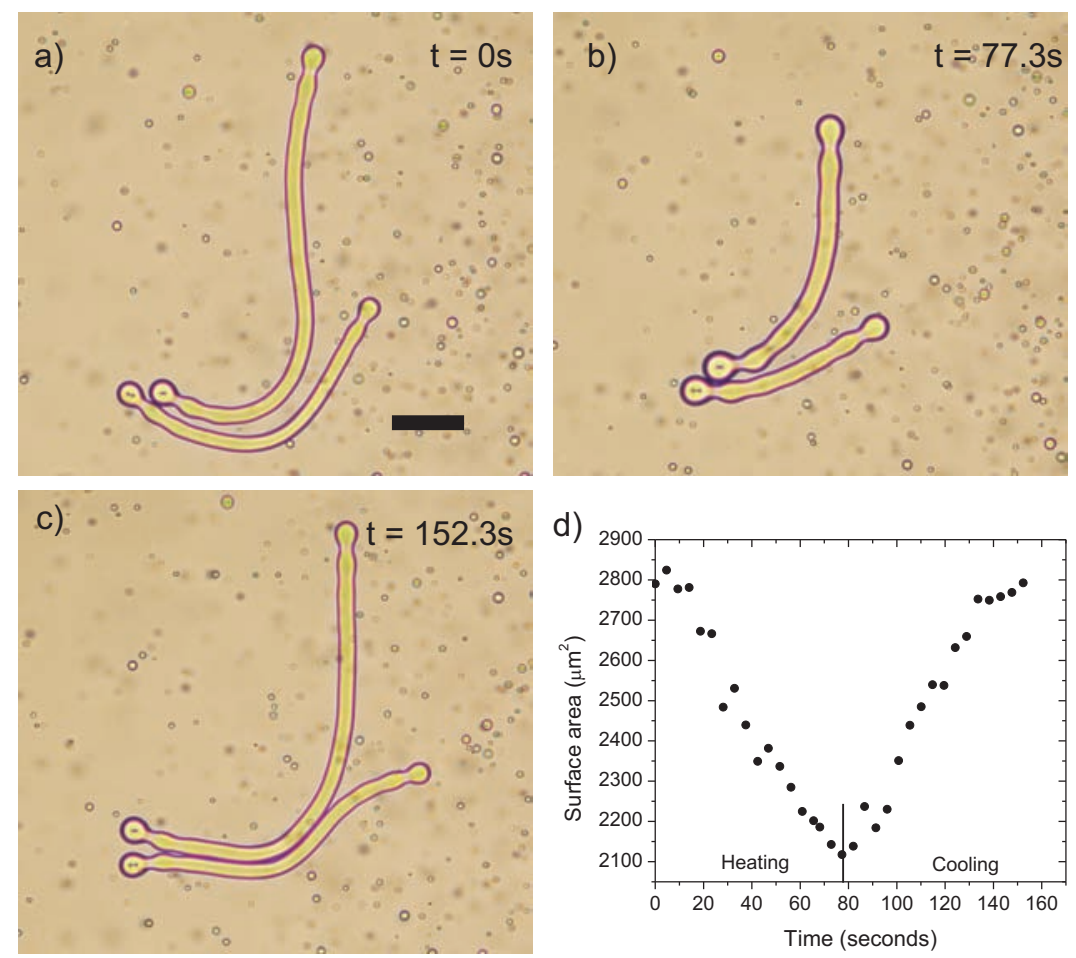

Figure 40: Effect of temperature on the surface area of a nematic filament. (a-c) The series of pictures represents a nematic filament in a CTAB $0.75 \mathrm{mM}$ solution at $35^{\circ} \mathrm{C}, 36.2^{\circ} \mathrm{C}$ and $35^{\circ} \mathrm{C}$ respectively. The filament contains $3 \mathrm{wt} \%$ monolein concentration in its bulk. Scale bar in image a: $25 \mu \mathrm{m}$ (d) The surface area of the upper filament in the above pictures is continuously tracked and plotted as a function of time. Heating/cooling rate is $0.1^{\circ} \mathrm{C} / \mathrm{min}$.

even after the nematic droplets' transformation into a filament. This elastic driven elongation of a nematic droplet may produce small daughter nematic droplets at the growing end of the nematic filament. As soon as a daughter droplet is separated from its parent nematic filament, a point defect spontaneously forms at the center of the droplet for topological reasons. These daughter droplets may produce further offspring (nematic droplets) through the same elongation process. This process is remarkably similar to the cell division processes.

Unidirectional growth of a nematic filament produces a hypothetical body with a firstgeneration arm. But a nematic droplet does not necessarily grow in a single direction. Multidirectional growth is also observed very frequently. The probability of finding droplets with 1 arm $>2$ arms $\gg 3$ arms $\gg 4$ arms. Nematic droplets with more than four first generation arms have not yet been observed. It is unknown whether a radial droplet with five first-generation arms or more is topologically possible. Fig. 41 shows the nematic droplets with different numbers of first-generation arms. It is also clear from Fig. 41 that the point defect is still present at the interjection of first-generation arms. These first-generation arms can further split and produce next generation arms. This process of splitting can continue indefinitely and produce complex structures as shown in Fig. 41(e-f).

The existence of a smectic-A phase below the nematic phase for $8 \mathrm{CB}$ provides an opportunity to study the effect of a Nematic - Smectic-A transition $\left(T_{N-S m A}\right)$ on the nematic filaments. As soon as the temperature of the system approaches the $T_{N-S m A}$, the nematic filament spontaneously breaks into equal-sized droplets. The transformation of the nematic filament into a smectic filament is never observed. A similar phenomenon is observed at the Cholesteric - Smectic-A phase transition by Lavrentovich and Nastishin [111]. Another 

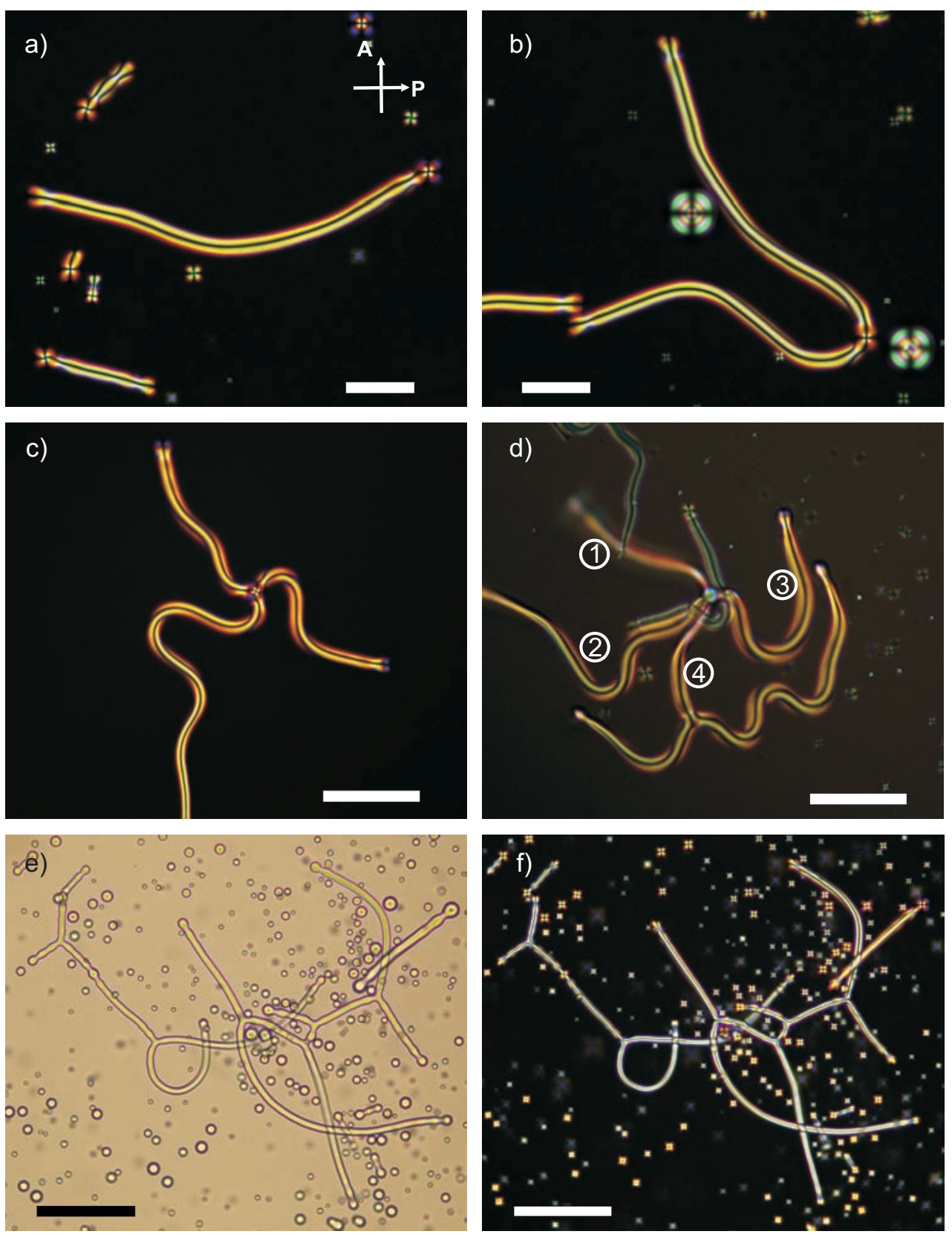

Figure 41: Observed structural distortions of a radial $8 C B$ nematic droplet with a hedgehog defect at the center. (a-b) Distorted radial droplets with one and two first generation arms respectively. Scale bars: $25 \mu \mathrm{m}$. (c-d) Distorted radial droplets with three and four first generation arms respectively. Scale bars: $50 \mu \mathrm{m}$. (e-f) A white light and a cross polarizer image of complex structural patterns produced due to the further splitting of first generation arms. Scale bars: $50 \mu \mathrm{m}$.

simple experiment is carried out by varying the monoolein concentration in $8 \mathrm{CB}$ but keeping the CTAB concentration in the aqueous phase constant at $0.5 \mathrm{mM}$. This time, a sandwich cell is prepared at $60^{\circ} \mathrm{C}$ and then the temperature is quenched to $T_{N-S m A}$. Nematic filaments start to grow at $T_{N I}$ and eventually all the filaments spontaneously break into droplets at $T_{N-S m A}$. As shown in Fig. 42 , the average smectic droplet size clearly depends on the initial monoolein concentration in the $8 \mathrm{CB}$ droplets. This observation also indirectly demonstrates that the average cross-sectional diameter of the nematic filaments at a given temperature decreases with increasing monoolein concentration. 


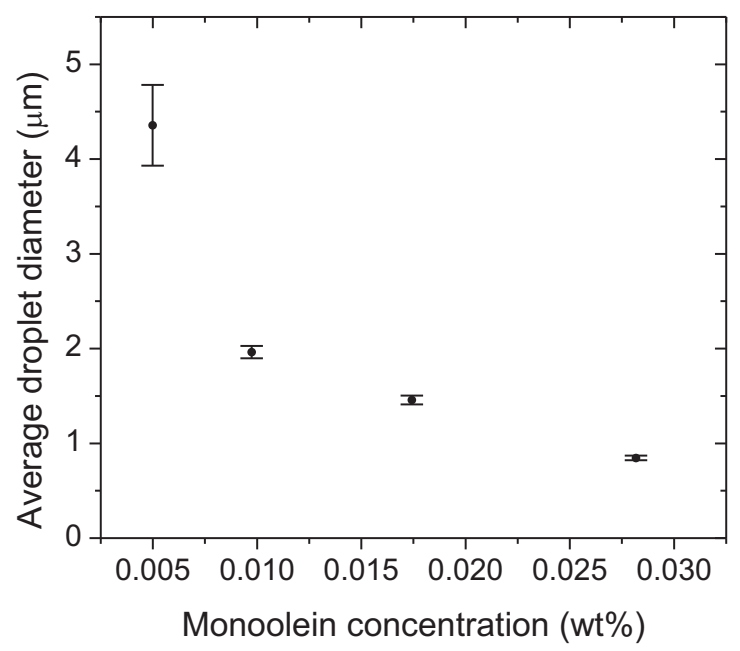

Figure 42: Average smectic droplet size vs monoolein concentration. The average size of the smectic droplets formed due to the breakage of the nematic filaments at $T_{N-S m A}$ decreases with an increase in the initial monolein concentration in the $8 \mathrm{CB}$ droplets' bulk. 


\subsection{Smectic liquid crystals in aqueous ionic mi- celler solutions}

The behavior of the thermotropic fluid smectic LCs (smectic-A, smectic-C and smectic-C*) in aqueous ionic surfactant solutions is investigated. The positional order (molecular layered structure) in smectic liquid crystals has a striking influence on their interaction with ionic micellar solutions. Smectic liquid crystals in aqueous ionic micellar solutions spontaneously show the formation smectic filaments at the interface which resemble myelin figures observed in lyotropic lamellar systems $[39,55,56,114-143,143-155]$. Detailed structural investigation of the smectic filaments is undertaken. The effect of various parameters on the growth behavior and the stability of these filaments are discussed. Smectic droplets can also selfpropel themselves in aqueous ionic micellar solutions but in a very different way than nematic and isotropic droplets do. Smectic filaments are strongly birefringent and their refractive indices are larger than that of the surrounding aqueous medium. Thus, the smectic filaments show interesting optical properties which may lead to soft matter photonic applications. Light guiding and whispering gallery mode lasing in a plane perpendicular to the major axis of the smectic-A filaments are demonstrated. Owing to the helical arrangement of the molecules in smectic- $\mathrm{C}^{*}$ filaments, photonic bandgap light guiding is also observed. The differences and similarities between the properties of thermotropic smectic systems and lyotropic lamellar systems are discussed.

\subsubsection{Lyotropic lamellar liquid crystals: Myelin figures}

The lyotropic lamellar phase $\left(L_{\alpha}\right)$ is the first ever liquid crystalline phase observed. A whitish soft substance, myelin, which is present on the surface of axons, was extracted and put into contact with water by Virchow and Mettenheimer in the 1850s $[116,156]$. The myelinwater interface is unstable and produces spectacular life-like cylindrical structures. These cylindrical structures are named after myelin and called myelin figures. Myelin is a lipid-rich substance in which lipids constitute $70 \mathrm{wt} \%$ of dry myelin and water constitutes $40 \%$ of the total weight [157]. At these high concentrations, lipids form bilayers in water ( $L_{\alpha}$ phase) [158]. Due to this reason, myelin has a multilamellar membrane structure. When these multilamellar membranes are in contact with excess water, they rearrange their structure and form myelin figures at the interface (Fig. 43). Myelin figures consist of multilamellar tubules of alternating lipid bilayers and water layers with a core axis of water. Later myelin figures are observed in various systems which have an $L_{\alpha}$ phase and no hexagonal phase at the investigated temperatures (Fig. 20) [56,127]. A necessary condition for observing myelin figures is that there must be a significant miscibility gap between the $L_{1}$ and $L_{\alpha}$ phases (i.e., lyotropic lamellar liquid crystal in the $L_{\alpha}$ phase must exhibit a low solubility in water). The smaller the miscibility gap, the harder it is to observe them with an optical microscope. If the miscibility gap is very small, a lyotropic lamellar liquid crystal dissolves in water without forming myelin figures at the interface. It is very interesting that no myelin figures are observed at a perfectly annealed lamellar liquid crystal-water interface (i.e., no defects in the liquid crystal) even though a large miscibility gap exists between these two phases [117]. However, if defects are created artificially in the annealed liquid crystal (using fibers or optical tweezers), myelin figures may start to grow spontaneously from the disordered regions. This shows that the presence of a large miscibility gap between the $L_{1}$ and $L_{\alpha}$ phases, and defects in the liquid crystal's bulk are necessary conditions for the formation of myelin figures at a lyotropic liquid crystal-water interface. The solvent 
phase does not need to be water as long as the above-mentioned necessary conditions are satisfied (tested solvents: glycerol, ethylene glycol and silicone oil). All myelin-forming systems (during the dissolution) reported so far are based on the lyotropic $L_{\alpha}$ phase.

Even after 160 years of discovering myelin figures, there is still no clarity regarding the sufficient conditions for the formation of myelin figures. Buchanan et al. suggested a "formation and growth by swelling" mechanism [55]. They suggested that excess water enters the lamellar phase and causes the interface to swell through the formation and growth of myelin figures. They also proposed that water exclusively enters the interface at the base of the myelin figures rather than entering through the tips by tracking the motion of tracer particles in the aqueous medium. According to this swelling mechanism, myelin figures must form and grow as long as there is a water flux into the lamellar phase. This mechanism contradicts one of the necessary conditions (defects in the lamellar phase). Huang et al., proposed a geometric model based on free energy minimization [129]. They showed that bilayer repeat spacing increases when a multilamellar disc is converted into a myelin, assuming the number of bilayers and the amount of material remains constant during the transition. An increase in the bilayer repeat distance reduces the bilayer repulsion, which in turn can compensate for curvature energy costs. Zou et al., suggested that hydration gradients or external solvent phase flows are required for the formation and retention of the myelin structures at the LC-water interface [117].

As there is a continuous water flux into the lamellar phase from the aqueous phase, analysis of the myelin figures formation and growth becomes very complicated. Thermotropic smectic liquid crystals provide a very simple alternative system for studying the myelin figures. Our experiments with thermotropic smectic liquid crystals suggest that interfacial tension between the LC phase-aqueous medium and the defects in the LC bulk play a key role in the formation, stability and growth of the myelin figures $[159,160]$. It is important to note that no report is available about the possible myelin figures with lyotropic lamellar
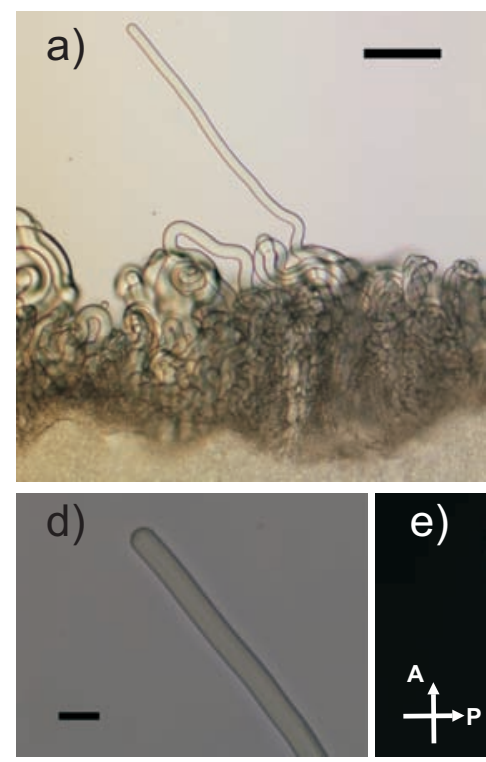
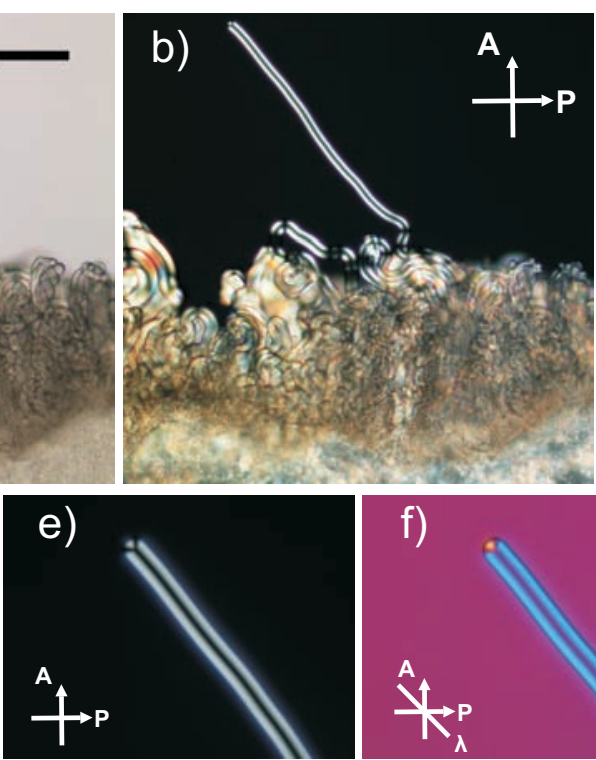

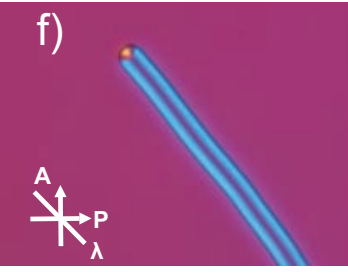

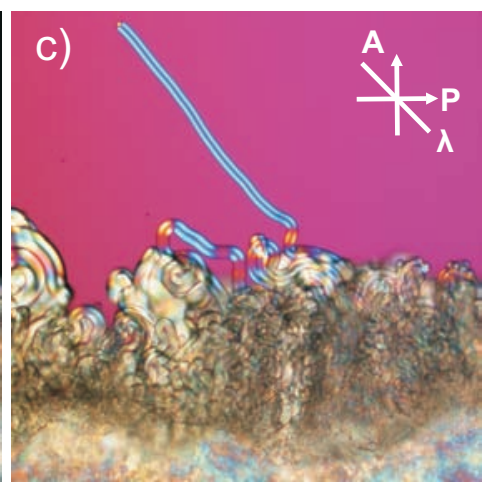

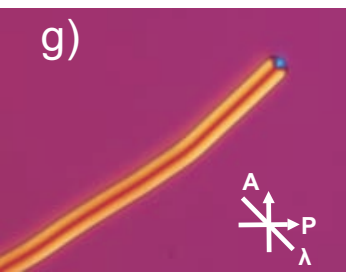

Figure 43: Instability at a lipid - water interface. (a-c) Polarizing microscopy images of myelin formed at the interface between $L_{\alpha^{-}}$Phosphatidylcholine and water. Top and bottom parts of images represent aqueous and pure lipid phases respectively. Scale bar in the image 'a' represents $100 \mu \mathrm{m}$. (d-f) Magnified images of the longest straight myelin figure in image 'a'. Scale bar in the image 'd' represents $20 \mu \mathrm{m}$. These images clearly show the presence of a defect core along the major axis of the myelin figure, as well as its internal structure. 
phases other than the $L_{\alpha}$ phase. Our experiments with thermotropic smectic liquid crystals suggest that all liquid crystalline phases with a molecular layered structure form myelin figures. Thus, we replaced the term 'myelin figures' with a more general term 'smectic filaments'.

\subsubsection{Three dimensional structure of the smectic filaments}

Thermotropic smectic liquid crystals (TSLCs) and aqueous ionic micellar solutions also satisfy the main necessary conditions for the formation of smectic filaments at their interfaces. TSLCs also contain a molecular-layered structure in the same way that lyotropic lamellar phases do. Thermotropic smectic phases can be formed by one-component systems (rod-like organic molecules), whereas lyotropic lamellar phases consist of at least two components. Owing to their high hydrophobic nature, TSLCs have a very limited solubility in aqueous ionic micellar solutions (rates of solubilization are very low).

No interfacial phenomenon exists at the TSLC-pure water interface. CTAB is used to reduce the interfacial tension values. As we increase the concentration of CTAB in the aqueous medium, the interfacial tensional value decreases. The liquid crystal surrounding the defects in the LC bulk diffuses into the surrounding aqueous solution (in the form of smectic filaments) in order to reduce the free energy of the system. The formation of smectic filaments is a direct result of the competition between the energy of LC bulk defects and the interfacial energy. As shown in Fig. 44(a-b), the interface of LC-CTAB solution expands in order to increase the order in the LC bulk. If the LC bulk is perfectly ordered before contact with the CTAB solution, no smectic filaments form at the interface (just as in the case of the perfectly ordered lyotropic $L_{\alpha}$ sample). However, this is not true for high CTAB concentrations, which can induce significant Marangoni stresses at the LC-aqueous solution interface. Strong Marangoni stresses create new defects inside the pre-ordered smectic liquid crystal and this may result in the formation of smectic filaments.

Almost all previous studies on lyotropic myelin figures used a sandwich method to observe and study the myelin figures. In this method, a small amount of lyotropic lamellar liquid crystal is sandwiched between two microscope glass slides which are separated by using spacers. LC is then put into contact with water by filling the remaining space between the glass slides with water via capillary action. The same sandwich method is used to produce thermotropic smectic filaments as shown in Fig. 44(a-b). Fluorescence confocal polarization microscopy (FCPM) is used to investigate the three-dimensional structure of the smectic filaments. As the smectic filaments are very sensitive to temperature, they continuously move in the aqueous medium or even change their structure while they are exposed to lasers (due to the lack of any kind of confinement). Thus, this method is not suitable for investigating the three-dimensional structure of smectic filaments. While looking for an experimental configuration that would offer a stronger confinement, we found that a single smectic filament can be grown in a glass capillary possessing a square cross section. A glass capillary is initially filled completely with an aqueous CTAB solution. Then, one end of the capillary is dipped into an LC droplet until a sufficient amount of LC replaces the aqueous solution (generally until it fills a few mm of the capillary). As shown in Fig. 44(c-f), a single smectic filament is achievable such that it exactly fits the square cross section of the capillary by tuning the system parameters (i.e., temperature, surfactant concentration and side length of the square capillary). The confinement in a capillary enables structural studies of a single filament using polarizing microscopy and FCPM.

The three-dimensional structure of the $8 \mathrm{CB}$ filaments (Smectic-A phase, $23^{\circ} \mathrm{C}$ ) is investigated. Fig. 45(a-b) shows polarizing microscopy images of a single 8CB filament. Only one 

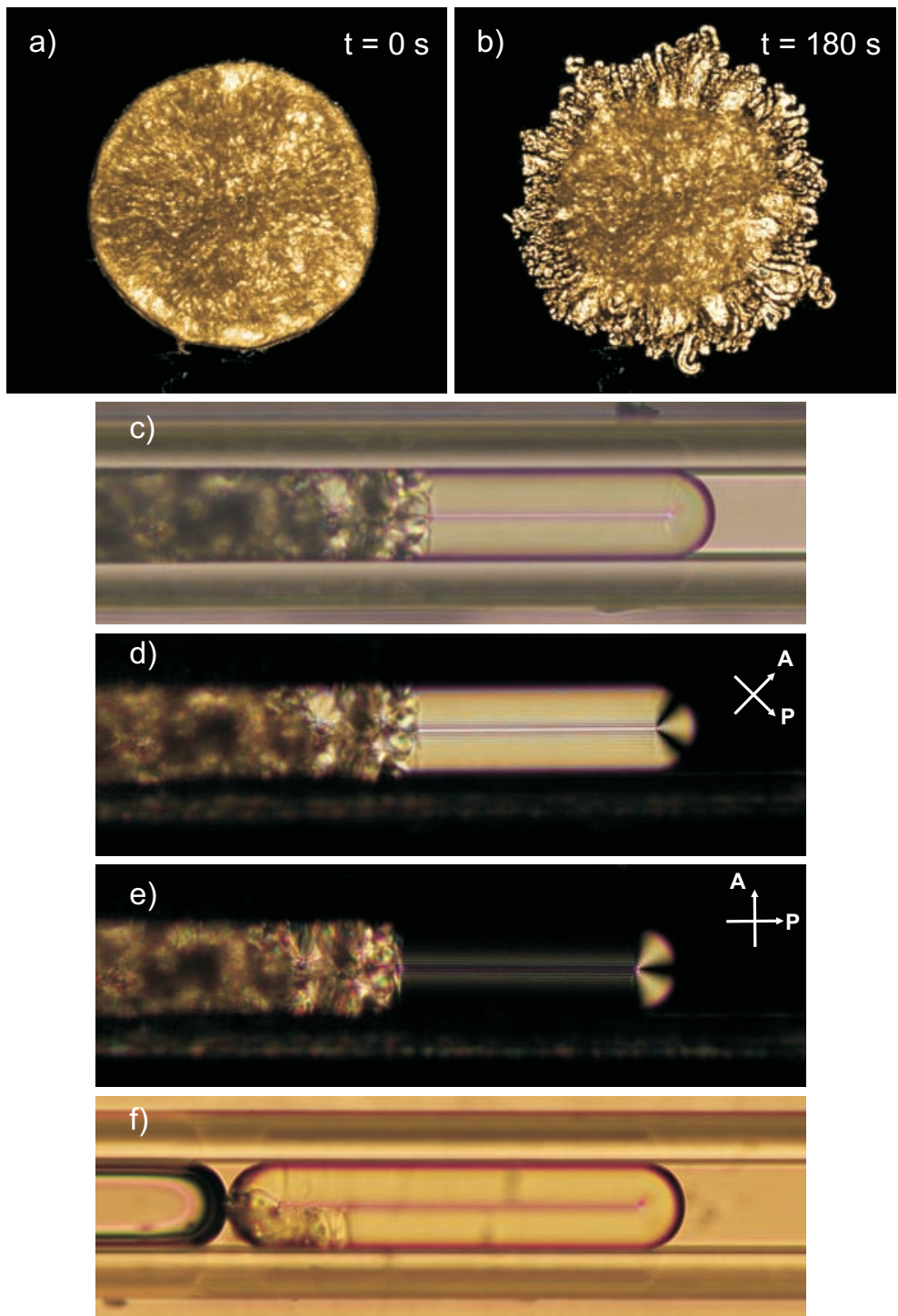

Figure 44: Employed methods for investigating filaments' formation and growth. (a-b) Sandwich method. Micrographs (crossed polarizers) of an $8 \mathrm{CB}$ droplet sandwiched between two glass plates separated by $100 \mu \mathrm{m}$ spacers. Two images show the sandwich $8 \mathrm{CB}$ droplet morphology before and after contact with CTAB $20 \mathrm{mM}$ aqueous solution. Horizontal width of the images is $3.85 \mathrm{~mm}$. (c-e) Capillary method: Polarizing microscopy images of a growing filament (from left to right) at the $8 \mathrm{CB}$-aqueous interface. Left and right hand parts of the capillary contain disordered $8 \mathrm{CB}$ and $\mathrm{CTAB} 10 \mathrm{mM}$ solution respectively. Inner width of the square capillary is $50 \mu \mathrm{m}$. (f) Almost all of the disordered $8 \mathrm{CB}$ is transformed into a well-ordered smectic filament (different experiment). Inner width of the square capillary is $50 \mu \mathrm{m}$.

way of arranging the smectic-A layers can possibly produce these microscopy images. An 8CB filament consists of concentric molecular layers wrapped around a line defect running along its major axis and $8 \mathrm{CB}$ molecules in each molecular layer are oriented along its layer normal (Fig. 45(c-d)). The concentric arrangement of layers can easily be deduced from the red wave plate images of a very thin filament (Fig. 45(e-f)). Filaments appeared blue and yellow in color when they are positioned parallel and perpendicular to the red wave plate respectively. For a positive birefringent uniaxial material, $8 \mathrm{CB}$, this combination of colors is possible if, and only if, the molecules inside a filament are oriented perpendicular 

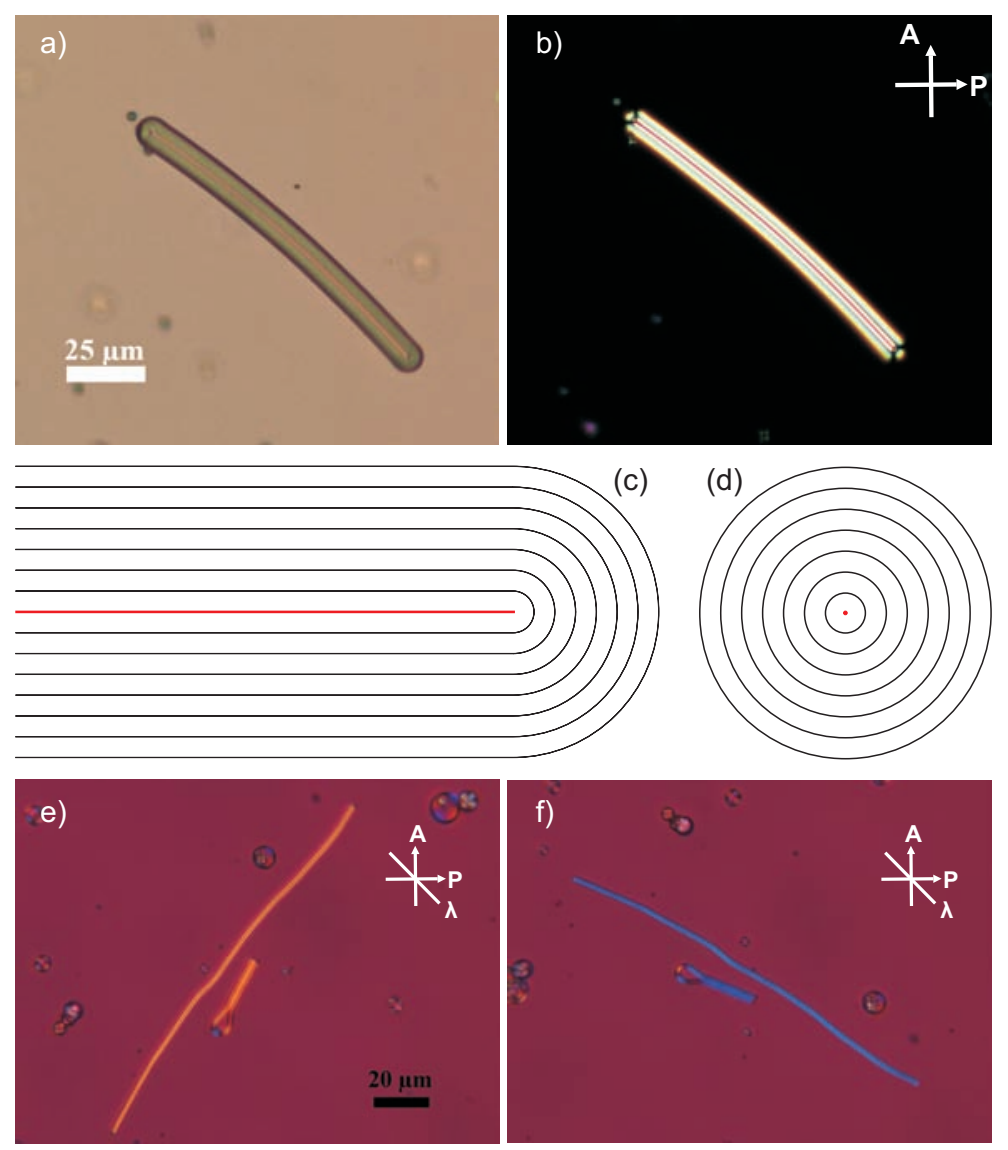

Figure 45: Polarizing microscopy images of smectic-A filaments. (a-b) A freely floating thick $8 \mathrm{CB}$ filament in CTAB $20 \mathrm{mM}$ aqueous solution. Due to the large birefringence of $8 \mathrm{CB}\left(\sim 0.16\right.$ at $\left.25^{\circ} \mathrm{C}\right)$, it is difficult to extract the internal director structure from the polarizing microscopy images of thick filaments. (c-d) Schematic drawings of the coaxial arrangement of the smectic layers in a filament. (c): Cross section parallel to the filament axis. (d): Cross section perpendicular to the filament axis. The red line or dot indicates the topological line defect. The rod-like LC molecules (not shown in the drawings) are oriented perpendicular to the layer planes and the filament surface. (e-f) Red wave plate images of a thin $8 \mathrm{CB}$ filament in CTAB $20 \mathrm{mM}$ solution suggest that the local optical axis is always aligned perpendicular to the surface of the filament.

to its surface. The concentric arrangement of the smectic layers is additionally confirmed by taking the FCPM images of an 8CB filament doped with Nile red (Fig. 46). Nile red molecules are known to orient along the LC molecules [161]. Thus, the brightest fluorescence must be observed when the polarization of the excitation light is set parallel to the LC director. If the polarization of the excitation beam is parallel to the major axis of the filament, maximum fluorescence intensity must be observed in the tip of the filament (if our expected arrangement of smectic layers is true). As shown in Fig. 46(c-h), experimental observations are in exact accordance with our expectations. The FCPM experiments also give direct proof of the cylindrical shape of the filaments (Fig. 46(e,h)).

Another important result of the FCPM experiments is that there is absolutely no indication of water inside the filament. This result provides insight into the more complicated process of lyotropic myelin figures. As mentioned earlier, lyotropic myelin figures are believed to be formed and grown due to the hydration gradient. It was observed that the creation of a defect on the surface of a pre-ordered lamellar liquid crystal shortly after immersing the sample in excess water produces myelin figures which grow from the defect 
a)

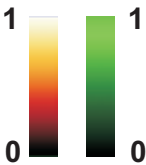

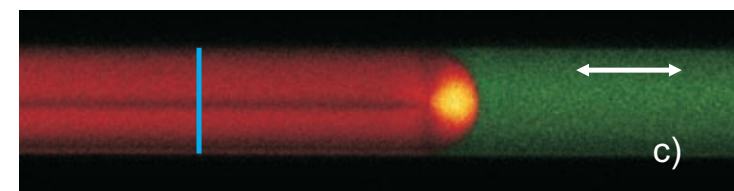

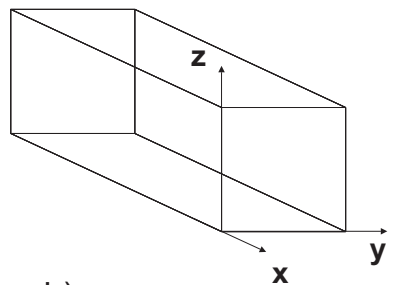

b)
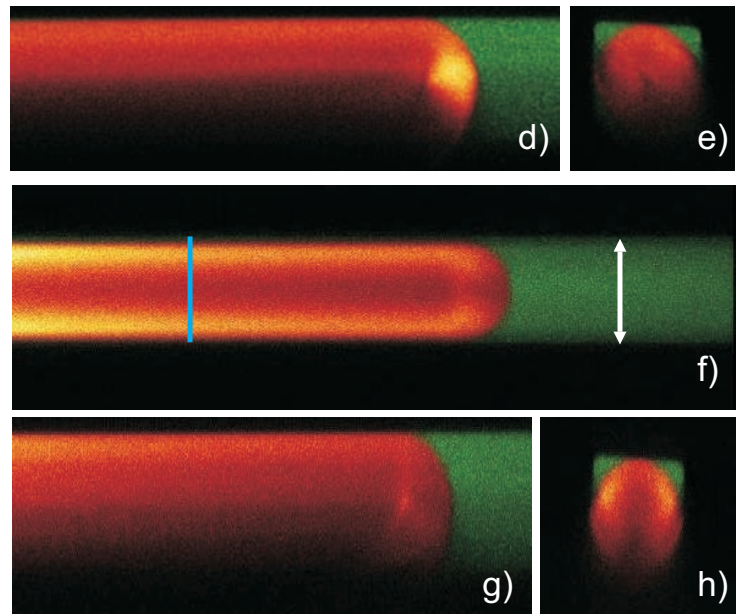

Figure 46: FCPM images of a smectic- $A$ 8CB (doped with nile red) filament. The 8CB filament growing in a square capillary (inner width $35 \mu \mathrm{m}$ ) filled with CTAB $20 \mathrm{mM}$ solution (doped with fluorescein). The red/yellow color indicates the fluorescence signal stemming from Nile red (smectic phase), and the green color indicates the fluorescence signal of fluorescein (aqueous phase). The color code describes the relative intensities of the Nile red and fluorescein signals respectively. (a) Schematic diagram of a square capillary. The capillary axis (growing direction of the filament) is along the $\mathrm{x}$ direction and the capillary is observed through the microscope along the $\mathrm{z}$ direction. (b) Cross section perpendicular to the capillary axis in a region that is filled with disordered bulk-like smectic 8CB (not shown here). (c) Cross section through the central region of the capillary along the xy plane (top view). The white double arrow indicates the polarization of the exciting laser light. The yellow color in the tip of the tube corresponds to a high intensity of the Nile red signal, indicating that in this region the dye molecules and the liquid crystal molecules are parallel to the polarization of the laser light. (d) Cross section through the central region of the capillary along the xz plane (side view). The decrease of the fluorescence intensity from top to bottom is a result of the relatively large thickness $(35 \mu \mathrm{m})$ of the sample. (e) Cross section perpendicular to the capillary axis along the blue line drawn in (c), demonstrating the cylindrical shape of the filament. It is to be noted that no bright spots are observed throughout the cross section. (f-h) Repetition of above experiment except that polarization of the exciting laser beam is perpendicular to the major axis of the filament. In this configuration, the tip of the filament exhibits the minimum fluorescence as expected.

region within a few seconds [117]. However, it was observed that these myelin figures stop growing and retract back into the LC sample after a few hours. It was suggested that retraction occurs once the water saturates the bilayer stack in the LC sample, thus removing the hydration gradient and internal stress. This may be false as our experiments suggest that interfacial tension (of $L_{\alpha}$-water interface) and defects (in the $L_{\alpha}$ phase) are the reasons for the formation and growth of the cylindrical structures (myelin figures). Hydration gradients do cause excess water to flow into the $L_{\alpha}$ phase and increase the water volume fraction. It is known that increasing the water content tends to increase the lateral repulsions between head groups of the amphiphile [158]. Lateral repulsions between head groups increase the interfacial area per amphiphile molecule which in turn increase the extent of water-hydrocarbon contact. Thus, interfacial tension between $L_{\alpha}$-water may increase over 
time and result in the retraction of myelin figures back into the LC bulk.

If there is no influx of aqueous solution into the thermotropic smectic filaments, the volume of the smectic liquid crystal must be conserved during the transformation from the disordered LC bulk into a smectic filament. This is proved by taking a known amount of liquid crystal in a square capillary and tracking the transformation process. Fig. 47 shows the volume data for a single filament growing in the capillary configuration: the magnitude of the volume change $\Delta V_{t}$, by which the tube has grown in a given time interval, equals exactly the amount of the volume change $\Delta V_{r}$, by which the volume of the reservoir of the disordered bulk-like smectic material has decreased, and the sum of the filament volume and the reservoir volume remains constant. These measurements, as well as the fluorescence confocal microscopy results, clearly indicate that the growth of the smectic filaments takes place without the incorporation of material from the aqueous phase (with the exception of the surfactant molecules adsorbed at the newly created smectic-aqueous interface). Rather it appears basically as a process of the rearrangement of the smectic layers.

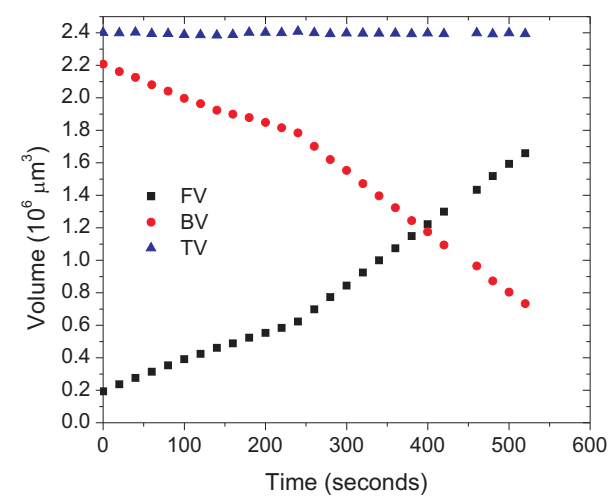

Figure 47: Volume data for a growing $8 C B$ filament in a square capillary. $8 \mathrm{CB}$ is in contact with the CTAB $40 \mathrm{mM}$ aqueous solution. FV: growing filament's volume, BV: volume of the disordered bulk-like $8 \mathrm{CB}$, and TV: sum of both volumes which remains constant. TV $=\mathrm{FV}+\mathrm{BV}$.

In the case of a nematic liquid crystal droplet in an aqueous micellar solution, there is a continuous water influx into the nematic droplet which results in the formation of aqueous daughter droplet chains. Thus, it is quite surprising that no water traces are found inside the $8 \mathrm{CB}$ smectic filaments during FCPM experiments. Smectic filaments can be stable if they are confined in a square capillary for at least several months. No water droplets are formed inside the smectic filaments (in a capillary) even after a prolonged exposure to the aqueous medium. This observation is in line with the results obtained for lyotropic myelin figures by Buchanan et al [55]. As mentioned earlier, they observed that the excess water from the surrounding aqueous phase exclusively diffuses into the lyotropic $L_{\alpha}$ phase at the myelin figures' roots. This means water flows into the lyotropic $L_{\alpha}$ bulk but not into the myelin figures (i.e., no water transport exists across the myelin-water interface). The flow of water continues until the bilayer stack becomes saturated. Similar behavior is observed for the $8 \mathrm{CB}-\mathrm{CTAB}$ system (sandwich method). As soon as an $8 \mathrm{CB}$ sandwich droplet is brought into contact with a CTAB aqueous micellar solution, smectic filaments form, but over time they either retract back into the LC bulk or break into smaller fragments (smaller filaments, droplets and vesicles). Even though no water is observed inside the filaments, water influx into the LC bulk is observed. Water enters into the LC bulk through the defects which exist at the interface. over time, several water islands can be observed along the defects inside the LC bulk. The size of each water island and the number of water islands increase 
over time. Fig. 48 provides the evidence for the formation of water islands inside the LC bulk. As shown in Fig. 48(c-d), water islands also form inside the LC droplets which are detached from the parent LC sandwich droplet. This phenomenon becomes much clearer if experiments are done using the capillary method. Initially a smectic filament is created inside a capillary as shown in Fig. 49(a). Small changes in the conditions such as temperature can destabilize the smectic filaments and induce defects inside them (Fig. 49(b)). There is no water inside the destabilized filament initially. But it is evidently clear from Fig. 49(c-e) that the water volume fraction increases inside the smectic filament continuously over time. Another simple experiment where disordered LC bulk and an ordered filament co-exist in a capillary provides further insight into the water transport. It is clear from Fig. 50 that the aqueous CTAB solution cannot diffuse through the ordered smectic-A region whereas a significant amount of water is transferred into the disordered smectic-A region.

Tilted smectic phases can also form filament-like structures at their interface with an aqueous CTAB solution. The macroscopic structure of the filaments is still the same as
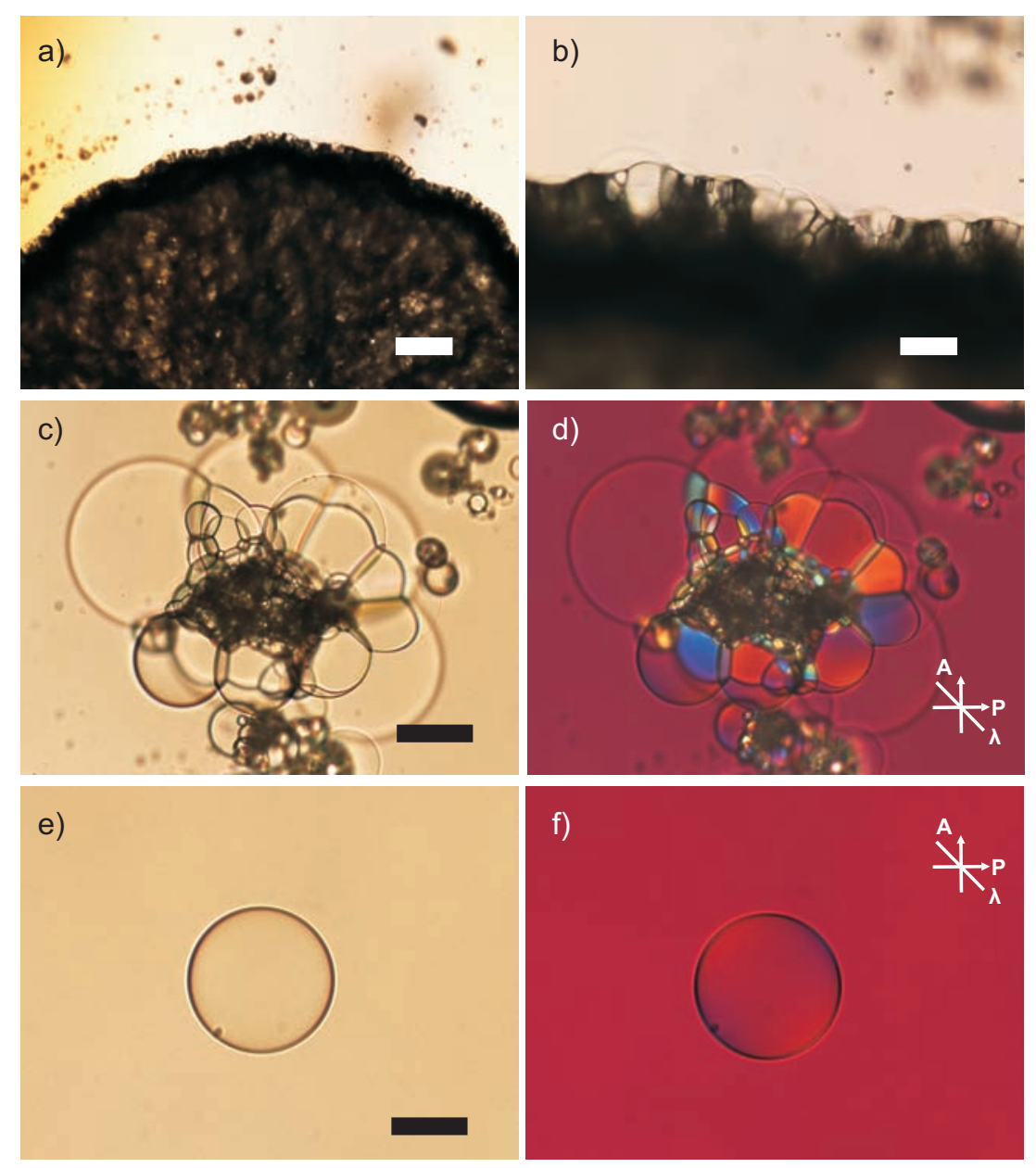

Figure 48: Optical micrographs of an 8cb sandwiched droplet. The droplet is in contact with CTAB 5 wt\% for two days. (a) Polarizing microscopy image of the 8CB-aqueous interface. Earlier formed smectic filaments are either transformed into tiny droplets or retracted back into the bulk of the original drop. Scale bar: $200 \mu \mathrm{m}$ (b) Magnified image of the 8CB-aqueous interface. Scale bar: $50 \mu \mathrm{m}$. (c-d) Polarizing microscopy image of a bulged 8CB droplet. Scale bar: $50 \mu \mathrm{m}$. (e-f) Some material can detach from the bulged droplet by forming multi-lamellar vesicles (smectic shell). The red wave plate image confirms the homeotropic arrangement of the smectic layers in the shell. Scale bar: $50 \mu \mathrm{m}$. 

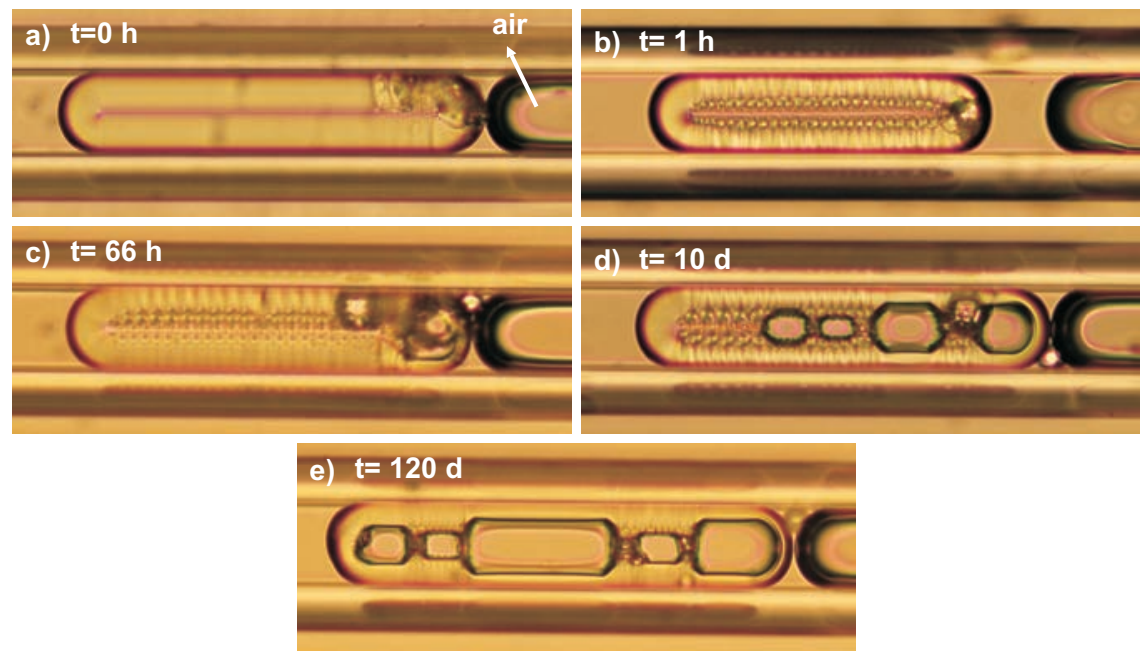

Figure 49: Water transport into a confined $8 C B$ smectic filament. (a) An 8CB smectic filament in a CTAB $20 \mathrm{mM}$ solution. (b) Filament lost its cylindrical shape and assumed a square cross-sectional shape after destabilization. (c-e) Volume fraction of the water inside the filament increases over time. Inner edge length of the square capillary is $50 \mu \mathrm{m}$. ' $\mathrm{h}$ ' and 'd' represents hours and days respectively.

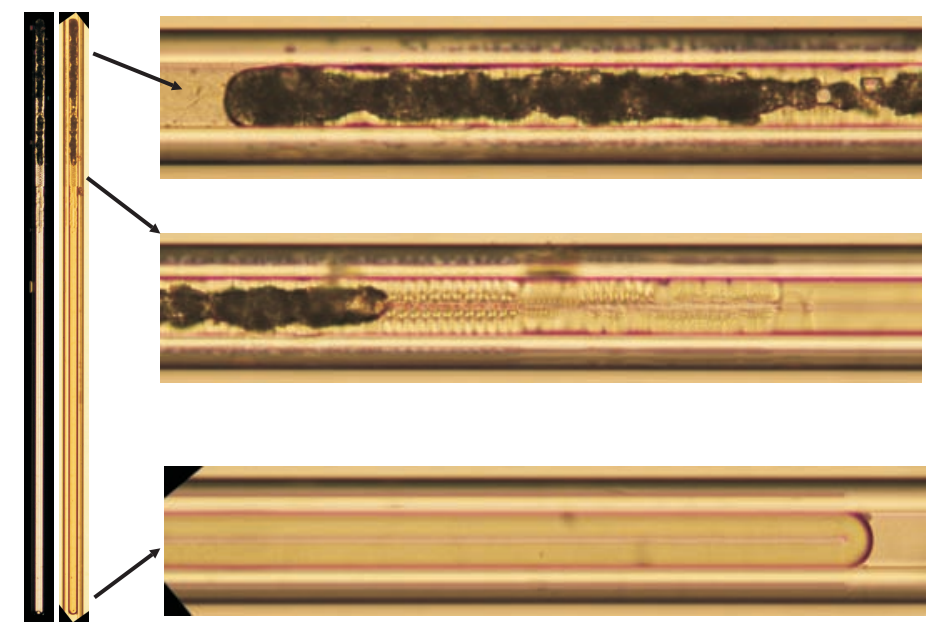

Figure 50: Eleven-months-old partially ordered smectic-A filament in CTAB $5 \mathrm{mM}$ aqueous solution. Inner edge length of the square capillary is $50 \mu \mathrm{m}$. Black colored region of the filament (disordered region) contains numerous water islands. No traces of water are found in the ordered region of the filament even after eleven months. This observation indirectly suggests that water, as well as CTAB, cannot diffuse through the perfectly ordered smectic layers.

that of smectic-A filaments (cylindrical objects with hemispherical caps at each end). A concentric organization of the molecular layers still persists with the only difference being the molecular arrangement inside the layers (Fig. 51). Smectic-C* filaments show interesting optical effects due to the helical arrangement of the molecules. An important phenomenon called 'selective reflection' occurs when the wavelength of incident light is of the same order as the pitch of the smectic- $\mathrm{C}^{*}$ liquid crystal. Fig. 52 shows a smectic- $\mathrm{C}^{*}$ filament under crossed polarizers at different temperatures. The pitch of the smectic-C* liquid crystals increases with temperature and becomes infinity at the Smectic- $\mathrm{C}^{*}$ - Smectic A transition temperature. Thus, different wavelengths from white light are reflected at different temperatures. Due to the selective reflection phenomenon, photonic bandgap light guiding is 


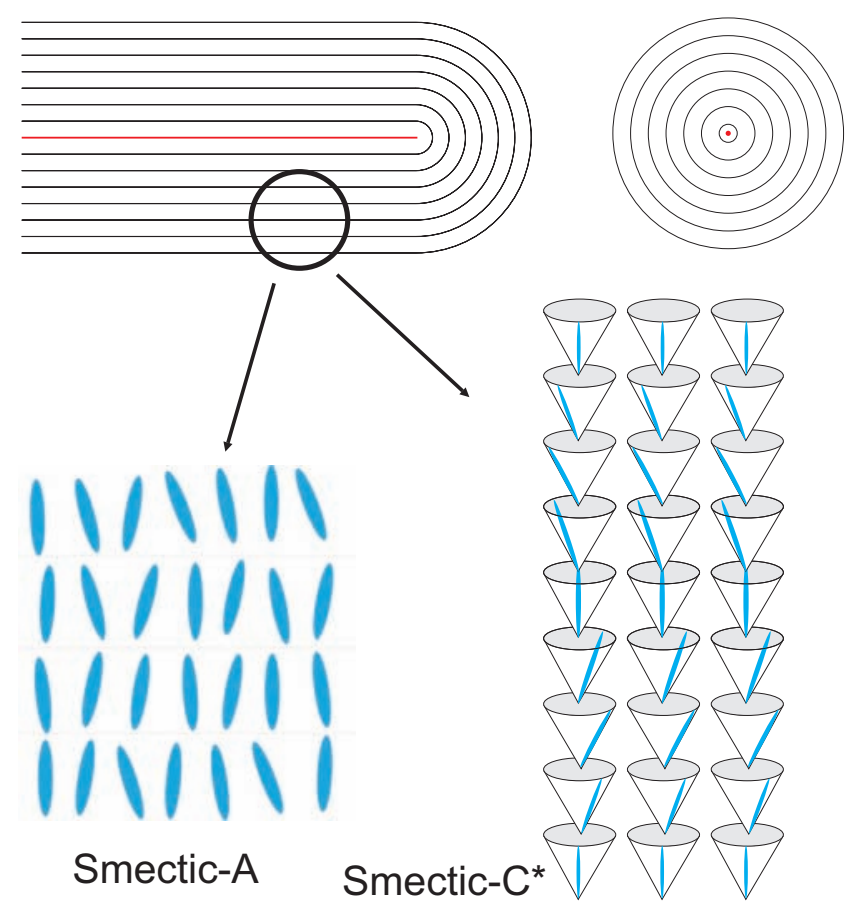

Figure 51: Comparison between smectic- $A$ and smectic- $C^{*}$ filaments' structures. Filaments in both phases contain the same cylindrical shape and a line defect along their major axes. However, their internal molecular arrangements are very different, which means they respond differently to externally applied fields.

observed in smectic- $\mathrm{C}^{*}$ filaments. More details about the light guiding in smectic- $\mathrm{C}^{*}$ filaments are provided in Section 3.2.7.3.

It is important to note that the aqueous $\mathrm{CTAB}$ solutions alone cannot destabilize the SLC-water interface for many materials. Of all the materials tried, only nCB (8CB-12CB) compounds could produce smectic filaments in CTAB solutions. The addition of a cosurfactant reduces the interfacial tension values considerably [14]. Thus, the addition of a small amount of a co-surfactant makes all the tested materials produce smectic filaments. In our case, a few weight percent of a non-ionic surfactant (monoolein or $C_{n} E_{n}$ ) is added to the liquid crystals. This method (addition of a co-surfactant) also works for producing nematic and cholesteric filaments.

It is observed that lyotropic myelin figures ( $L_{\alpha}$ phase) may bend to form single coils and double helices. As shown in Fig. 53(a-f), two different types of double helices are observed in lyotropic $L_{\alpha}$ systems. It was reported that myelin figures remain either uncoiled or tightly coiled (without deforming the cylindrical structure) [139]. Irregular coils (Fig. 53(g)) without a well-defined chirality are also observed along with regular helices. There is no detectable change in the volume or surface area of the myelin figures due to the coiling. Several theories for myelin coiling instabilities have been proposed. Lin et al. [146] reported that in the presence of $\mathrm{Ca}^{2+}$ ions, binary mixtures of cardiolipin and phosphatidylcholines readily form tightly-folded single coils and double helices. The coiling tendency of the myelin figures formed by charged amphiphiles increases significantly with increasing salt concentration. It is shown that weak $\mathrm{Ca}^{2+}$-mediated membrane-membrane binding energies can overcome curvature elastic energy and stabilize the coil configurations. Mishima et al. observed that egg-yolk phosphatidylcholine (egg-PC) forms double helices even in pure water [138]. Coiling instabilities are also observed during the dissolution of non-ionic surfactants in pure water [55]. Frette et al. observed that above a certain critical concentration of a specially-designed 

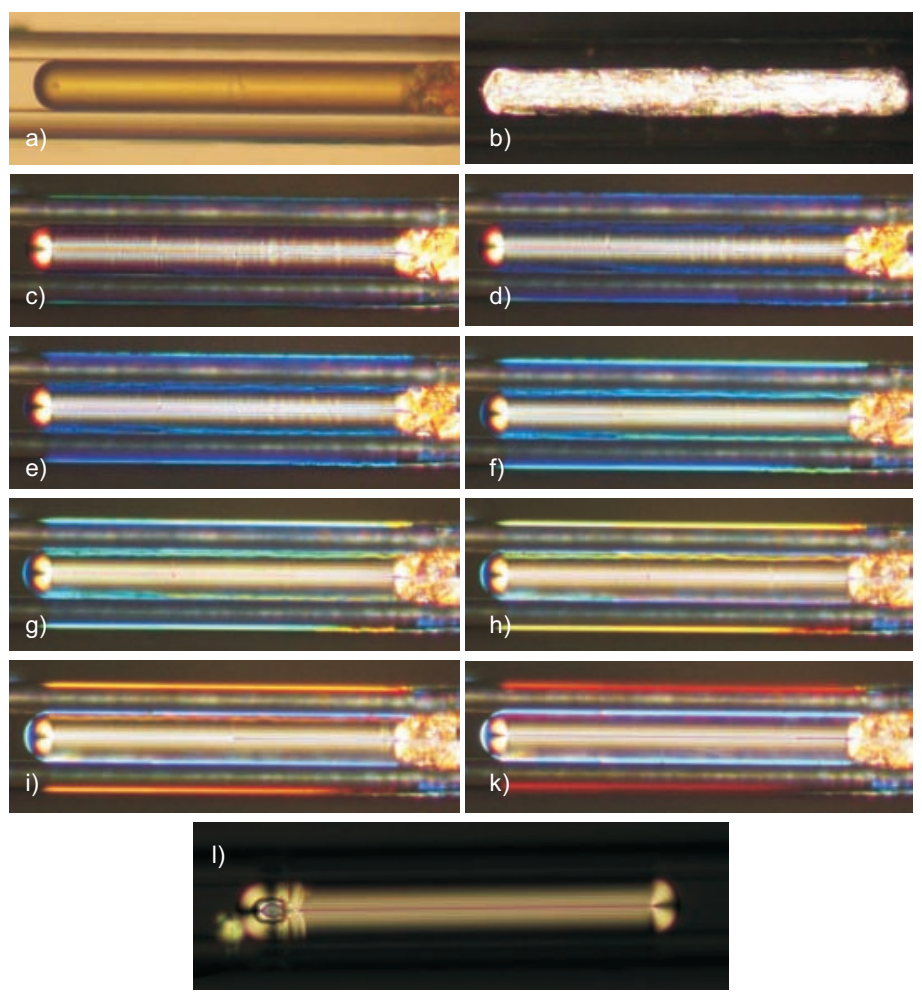

Figure 52: Crossed polarizer images of a C7 filament at different temperatures. (a) White light image of a $\mathrm{C} 7$ filament (smectic- $\mathrm{C}^{*}$ phase) in $50 \mu \mathrm{m}$ square capillary at $50^{\circ} \mathrm{C}$. (b) Crystallized $\mathrm{C} 7$ filament at $40^{\circ} \mathrm{C}$. (c-k) These optical micrographs show the $\mathrm{C} 7$ filament at temperatures (in ascending order) $47^{\circ} \mathrm{C}, 48^{\circ} \mathrm{C}, 49^{\circ} \mathrm{C}, 50^{\circ} \mathrm{C}, 51^{\circ} \mathrm{C}, 52^{\circ} \mathrm{C}, 53^{\circ} \mathrm{C}$ and $54^{\circ} \mathrm{C}$ respectively. Images are taken in transmission mode. Helical pitch of $\mathrm{C} 7$ increases with increasing temperature. Thus, wavelengths that get reflected from the surface of the filament increase with increasing temperature. Glowing colors of the capillary represent the reflected wavelengths from the surface of the filament. (l) Optical micrograph of a smectic-A filament. As the period of the structure is well below the visible wavelengths of light, selective reflection is not observed in the smectic-A filament.

polymer in the aqueous solution, a writhing instability sets in and myelin figures form single coils, double helices and many irregular structures. It was suggested that coiling is a result of a coupling between local bilayer curvature and polymer concentration [139]. Santangelo et al. proposed that myelin figures are unstable to coiling when they acquire a spontaneous curvature or when the equilibrium distance between membranes is decreased [142].

Thermotropic smectic-A liquid crystals (the thermotropic analogue of $L_{\alpha}$ phase) also show all the above coiling instabilities. $8 \mathrm{CB}$ generally does not produce any helically arranged smectic-A filaments in CTAB solutions. The addition of salts to the CTAB solutions significantly reduces the electrostatic repulsion between smectic-A filaments and invokes strong attraction between the filaments (Fig. 54(a)). Fig. 54 shows different types of coils produced by smectic-A liquid crystals. In an egg-PC-water system, the helix formation progresses at a slow rate of about 10 seconds per pitch [138]. The helix formation in 8CBwater-CTAB-salt systems can be extremely fast ( $<1$ second per pitch).

Sparingly water-soluble chiral amphiphiles can self-assemble into many more varieties of bilayer-sheet based microstructures [162-167]. The most interesting morphologies are ribbons (helical ribbons and twisted ribbons) and cylindrical tubules. It is observed that chirality is often expressed in the morphology of these curved aggregates. R- and S-enantiomers of chiral amphiphiles generally produce right- and left-handed aggregates respectively. In- 

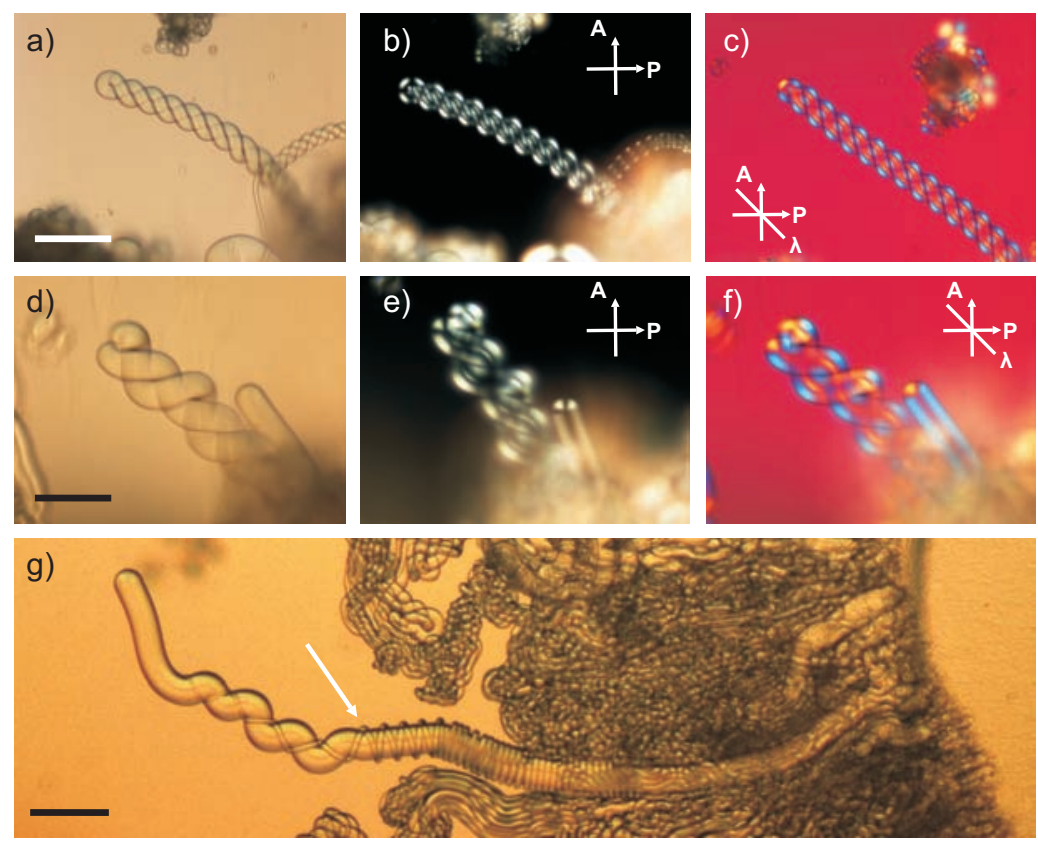

Figure 53: Optical micrographs of double helical myelin figures formed at lipid-aqueous interface. (a-c) Double helical structure formed by one myelin tube at a $L_{\alpha^{-}}$Phosphatidylcholine (PC) - pure water interface. Scale bar: $100 \mu \mathrm{m}$ (d-f) Double helical structure formed by two myelin tubes at a PC- pure water interface. Scale bar: $50 \mu \mathrm{m}(\mathrm{g})$ Irregular structure at a lipid mixture-aqueous interface. Lipid mixture consists of $80 \%$ PC and $20 \%$ PS (Phosphatidylserine). Aqueous solution contain $0.5 \mathrm{M} \mathrm{NaCl}$ and $80 \mathrm{mM}$ CTAB. Scale bar: 100 $\mu \mathrm{m}$.

terestingly, some achiral amphiphiles (also a few pure enantiomers) produce equal numbers of right- and left-handed aggregates. This clearly demonstrates that chirality is not essential for the formation of helices and tubules. In this case, it was suggested that curved aggregates form due to spontaneously broken chiral symmetry rather than due to a chiral molecular packing. Our experiments with thermotropic smectic-C liquid crystals also show that chirality is not necessary for the formation of helical structures. $8 \mathrm{OPhPy} 8$ in the smectic-C phase produces an equimolar mixture of right- and left-handed helical filaments (Fig. 55). There is no preferred diameter and pitch for the helical filaments. No ribbon-based aggregates are observed in smectic-C liquid crystals.

As with chiral amphiphiles, thermotropic smectic-C* liquid crystals form ribbon-based aggregates. As shown in Fig. 56, both twisted ribbons and helical ribbons are observed along with normal filament-like structures (both straight and helical). The ratio of right-handed to left-handed helical ribbons (or filaments) is yet to be determined. Helical structures with different pitches and diameters are observed. In the case of amphiphiles, it is observed that helical ribbons slowly evolve into cylindrical tubules. This transition is not yet observed with thermotropic smectic-C* liquid crystals. However, helical structures do further reorganize and form super helical structures as shown in Fig. 56(d). Detailed structural analysis of the super helical structures is not yet completed. 


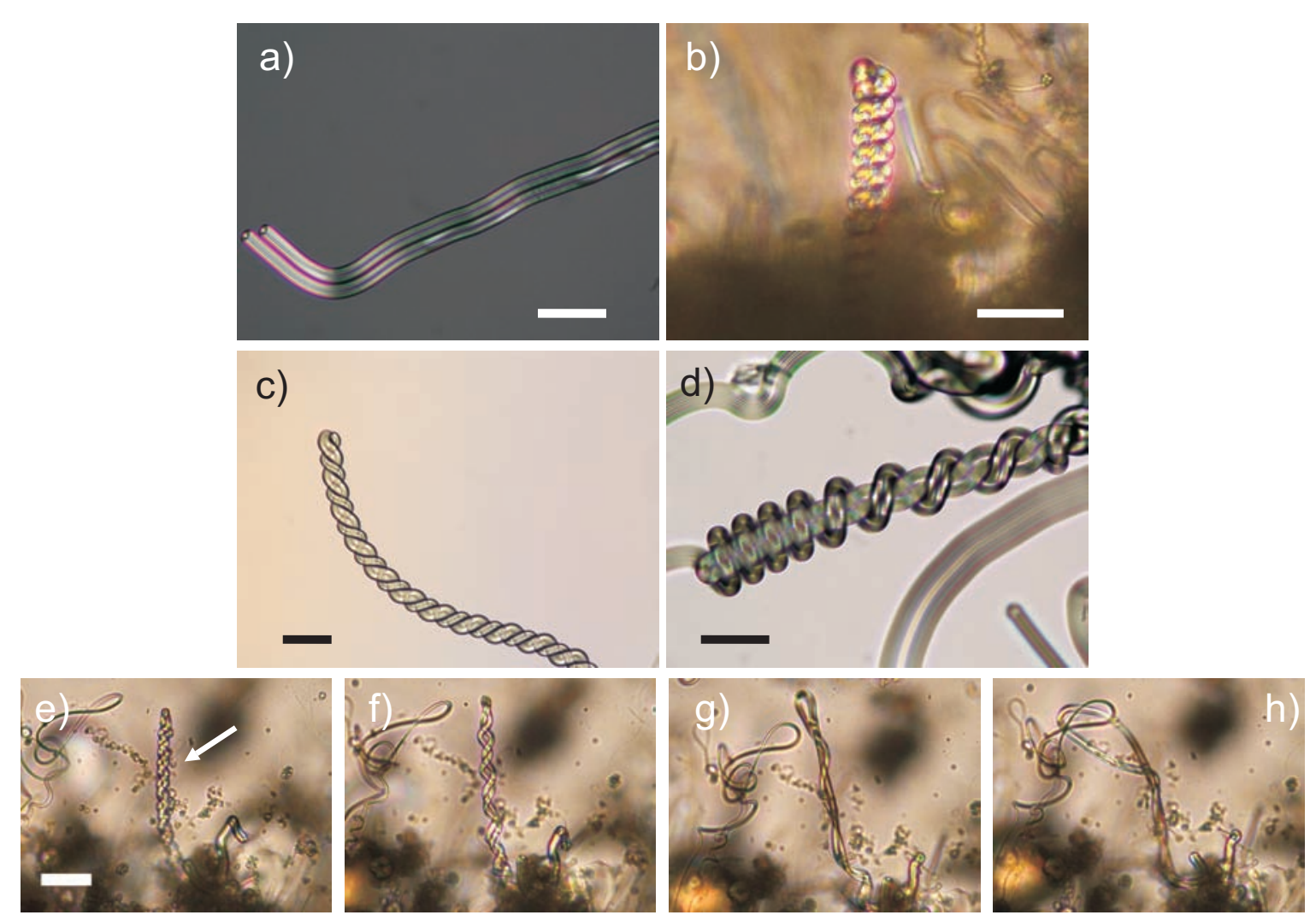

Figure 54: Optical micrographs of various structures formed by thermotropic smectic-A filaments. These structures are observed in a 12CB-CTAB $10 \mathrm{wt} \%$ system. The aqueous CTAB solution also contains $0.5 \mathrm{M} \mathrm{NaCl}$. (a) Strong attraction between two filaments causes them to grow together. It requires a significant amount of energy (using optical tweezers) to separate them. Scale bar: $50 \mu \mathrm{m}$. (b) A single stranded coil with an open end. Scale bar: 25 $\mu \mathrm{m}$. (c) A double stranded helical structure formed by two filaments. Scale bar: $50 \mu \mathrm{m}$. (d) An example for irregular coils. Scale bar: $50 \mu \mathrm{m}$. (e-h) A double helix formed by a single filament. This series of images (left to right) shows the unwinding of the helix. Scale bar: $25 \mu \mathrm{m}$. 

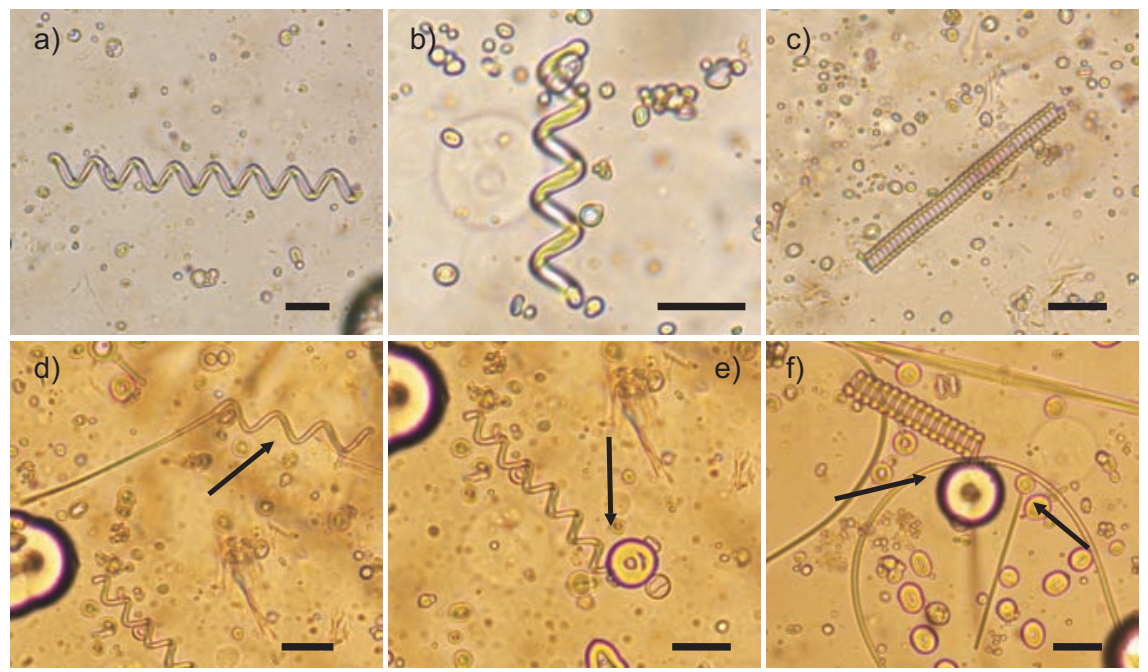

Figure 55: Optical micrographs of various thermotropic smectic- $C$ filaments. 8OPhPy8 is doped with $1 \mathrm{wt} \%$ monoolein and the aqueous solution contains $1.5 \mathrm{mM}$ CTAB. Scale bars in all images represent $15 \mu \mathrm{m}$. Black arrows in images d-f point to the regions of interest. (a-b) Helical filaments with opposite handedness. (c) Tightly packed low pitch helical filament. (d) Partially curved smectic-C filament. (e) Smectic-C droplet with a high pitch helical filament as a tail. (f) One smectic droplet contains a straight filament as a tail whereas another droplet contains low pitch helical filament as a tail. It is important to note that normal straight filaments (like smectic-A filaments) are the most frequently observed structures.
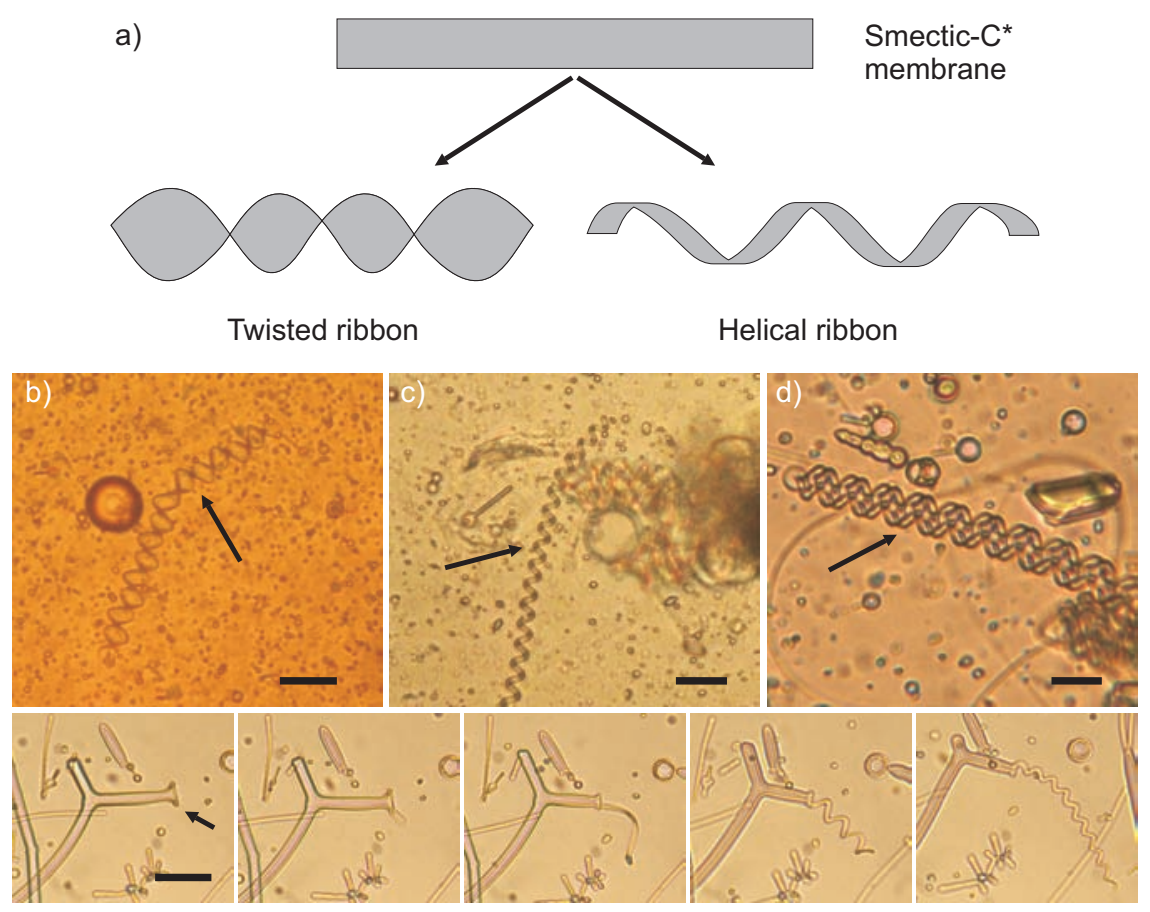

Figure 56: Optical micrographs of the observed structures in a smectic- $C^{*} L C$-water system. The LC mixture contains $75 \mathrm{wt} \% \mathrm{C} 7$ and $25 \mathrm{wt} \%$ 8.0.6. This LC mixture is doped with $1 \mathrm{wt} \%$ monoolein. The aqueous solution contains $1.5 \mathrm{mM}$ CTAB. (a-c) The smectic-C* membrane can morph into any one of the two ribbon structures. Scale bars in b and c images represent $25 \mu \mathrm{m}$. (d) A superhelical structure formed by a helical ribbon. Scale bar: $25 \mu \mathrm{m}$. Bottom row of images shows the expanding smectic- $\mathrm{C}^{*}$ membrane (left to right). The membrane expands and produces a helical ribbon. Scale bar: $10 \mu \mathrm{m}$. 


\subsubsection{Growth behavior of thermotropic smectic-A filaments}

Both sandwich and capillary methods are employed to study the growth behavior of smecticA filaments. $8 \mathrm{CB}$ and aqueous $\mathrm{CTAB}$ micellar solutions are used to produce smectic-A filaments. $8 \mathrm{CB}$ is very convenient for these experiments as it is in the smectic-A phase at room temperature. The effect of various parameters on the filament growth behavior, such as surfactant concentration and temperature, is discussed below.

\subsubsection{Sandwich method}

For a sandwich experiment, a small amount of $8 \mathrm{CB}$ is sandwiched between two glass slides which are separated by thin Mylar spacers. Then, the remaining empty space between the glass slides is filled via capillary action with an aqueous CTAB solution, thereby bringing the smectic liquid crystal into contact with the CTAB solution. Fig. 57 shows a typical time evolution of the $8 \mathrm{CB}$-aqueous interface. Smectic-A filaments grow almost uniformly in all directions at the expense of the bulk of the original $8 \mathrm{CB}$ droplet. However, smectic-A filaments do not grow indefinitely. The growth rate of the filaments gradually reduces over time and completely stops after a certain amount of time. And as shown in Fig. 57, subsequently, all the filaments lose their order and merge with their respective neighbors. This is due to the finite amount of surfactant in the aqueous solution. The micellar solubilization of the $8 \mathrm{CB}$ sandwich droplet in the surrounding aqueous solution and the transfer of aqueous solution into the $8 \mathrm{CB}$ sandwich droplet simultaneously continue with the growth dynamics of the filaments. Due to this reorganization of the components of the system, active micelles that are necessary to replenish the $8 \mathrm{CB}$-aqueous interface gradually decrease. As a result, the interfacial coverage of the CTAB reduces over time, which in turn increases the interfacial tension as well as decreases the electrostatic repulsions between the neighboring filaments. The increase in interfacial tension makes these filaments unstable and they gradually lose their respective ordered structures. Subsequently, the disordered neighboring filaments merge with each other as soon as the Van der Waals attractions between them
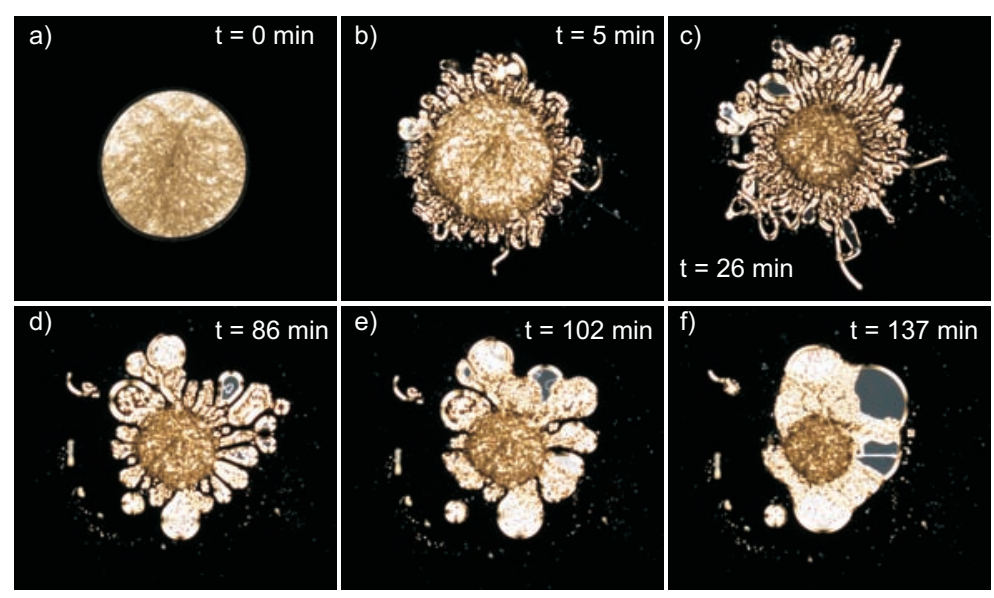

Figure 57: Sandwich method - time evolution of the 8CB-aqueous interface. An 8CB droplet is sandwiched between two glass plates separated by $100 \mu \mathrm{m}$ spacers. Aqueous solution contains $5 \mathrm{mM} \mathrm{CTAB}$. (a) The smectic droplet just before contact with the $5 \mathrm{mM} \mathrm{CTAB}$ solution. (b-f) Filament-like structures are observed at the LC-aqueous interface initially and they continue to grow for a while. But they eventually cease to grow and merge with each other to again produce bulk-like $8 \mathrm{CB}$. Horizontal width of all the images is the same $(3 \mathrm{~mm})$. Images are taken under crossed polarizers. 
overcome the electrostatic repulsions.

\subsubsection{Growth rates of smectic-A filaments: Effect of CTAB concentra- tion}

To quantify the growth of the smectic filaments, both the droplet-filament interface and the outer edge of the total birefringent region (i.e., the total area occupied by filaments and the remaining part of the original $8 \mathrm{CB}$ droplet) are approximated by circles. The difference between their radii, i.e., the width $L$ of the filament-filled region, is determined as a function of the time $t$ which has elapsed since the initial contact between the smectic liquid crystal and the surfactant solution. A sample filament growth behavior at an 8CB - $20 \mathrm{mM} \mathrm{CTAB}$ solution interface is shown in Fig. 58(a). After an initial period of about 10 to 20 seconds (ballistic regime), $L$ grows for a longer time interval as $t^{0.5}$ (diffusive regime) until, after about 500 seconds, the data suggest a slowing of the growth (sub-diffusive regime). Eventually filaments cease to grow as is the case shown in Fig. 57. A diffusive growth behavior for a longer period is a common observation when lyotropic myelin figures are studied using the sandwich method $[118,153]$. As mentioned earlier, it is attributed to the collective diffusion of surfactant molecules induced by the hydration gradient in the $L_{\alpha}$-water contact zone.

We investigated the diffusional regime for different CTAB concentrations. Diffusional regimes exist for all investigated CTAB concentrations $(5 \mathrm{mM}-80 \mathrm{mM})$. The dependence
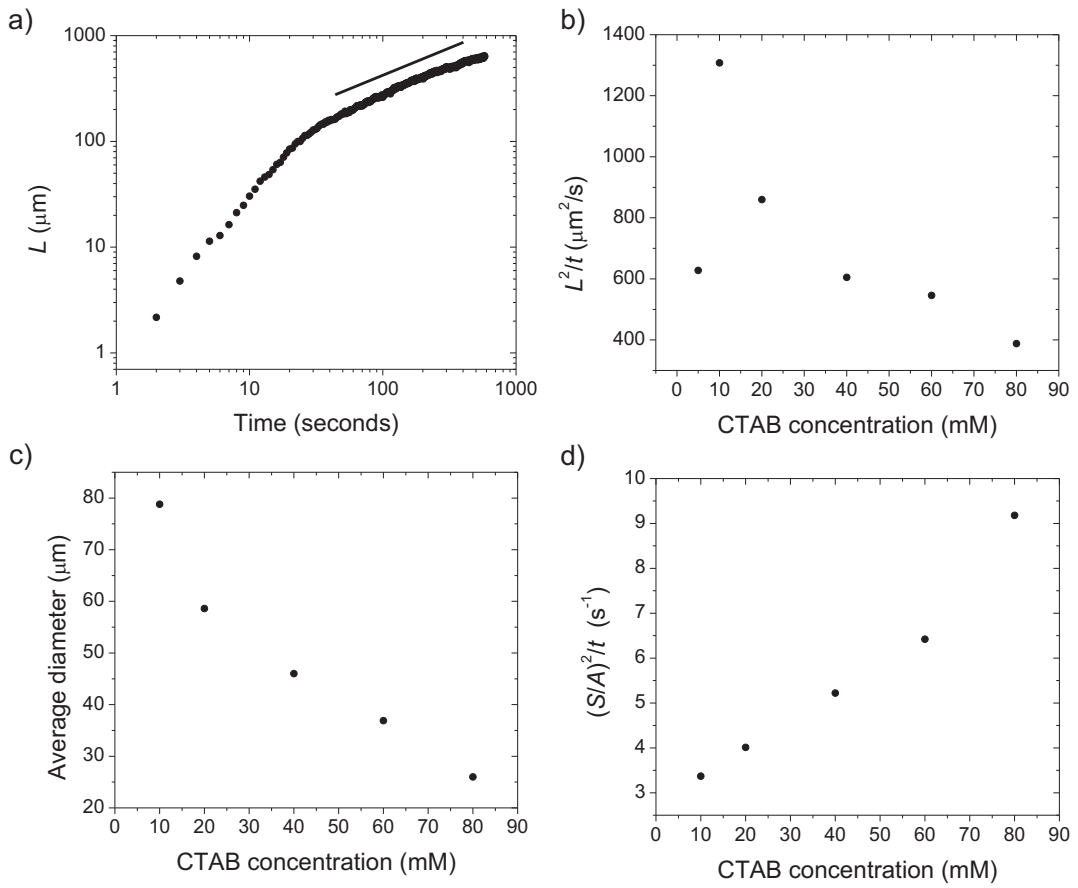

Figure 58: Sandwich method - effect of CTAB concentration on the growth behavior of smectic-A filaments. $100 \mu \mathrm{m}$ spacers are used to prepare sandwich cells. (a) Growth behavior of $8 \mathrm{CB}$ filaments in CTAB $20 \mathrm{mM}$ solution. The solid line represents a hypothetical line (slope $=0.5$ ) which acts a guide for identifying the diffusional regime. (b) Slopes $\left(L^{2} / t\right.$ ) from diffusional regimes of growth plots obtained for various concentrations of CTAB. (c) Average diameter of the filaments observed as a function of CTAB concentration. The average diameter value for $5 \mathrm{mM}$ concentration lies above the spacers' thickness $(100 \mu \mathrm{m})$. Thus, experiments are conducted only for higher concentrations. (d) This plot clearly shows that the $8 \mathrm{CB}$-aqueous interfacial area is higher for higher concentrations at any time of observation. 
of $L^{2} / t$ on CTAB concentration is shown in Fig. 58(b). Apparently, the growth rate first increases with increasing CTAB concentration, is maximal for $10 \mathrm{mM}$ concentration, and then decreases with increasing values of CTAB concentration. On the contrary, one would expect that the growth becomes faster with increasing CTAB concentration. This is due to the fact that the interfacial tension decreases with increasing CTAB concentration. This apparent contradiction is resolved after investigating the cross-sectional diameters of the filaments. The average diameter of the smectic filaments decreases with increasing CTAB concentration (Fig. 58(c)) while the packing density does not show an obvious change. In other words, with increasing CTAB concentration, the average diameter of the filaments decreases but their number density significantly increases. These observations suggest that, instead of their length, the surface area of the filaments per unit area of the initial smectic LC-aqueous interface should be used as a measure of the filament growth. If we assume that the type of packing of filaments remains the same when the filament diameter changes, we can simply divide the surface area $S$ of a filament (calculated from $L$ and $D$ assuming a cylindrical geometry) by the area $A$ of the cross section of the filament in order to obtain an appropriate measure. A plot of $(S / A)^{2}$ vs. $t$ has, of course, the same shape as $L^{2}$ vs. $t$ but it quantifies the growth behavior of the total surface area instead of the length of the filaments. Fig. 58(d) shows the result when the slope $L^{2} / t$ is replaced by the slope $(S / A)^{2} / t$, indicating now that the growth rate of the filaments (i.e., the growth rate of $8 \mathrm{CB}$-aqueous interfacial area) increases monotonously with increasing CTAB concentration.

\subsubsection{Growth rates of smectic-A filaments: Effect of temperature}

Fig. 59 shows the effect of temperature on the growth of $8 \mathrm{CB}$ smectic-A filaments. The concentration of CTAB and spacers' thickness are fixed at $40 \mathrm{mM}$ and $100 \mu \mathrm{m}$ respectively. Snapshots of the $8 \mathrm{CB}-\mathrm{CTAB} 40 \mathrm{mM}$ system at different temperatures are taken five minutes after the initial contact between the $8 \mathrm{CB}$ sandwich droplet and an aqueous micellar solution. The width $L$ of the filament-filled region is calculated as in the previous Section 3.2.3.2. The average length of the filament $(L)$ decreases with increasing temperature at any given time. In other words, as the system temperature approaches the smectic to nematic transition temperature $T_{S m A-N}\left(33^{\circ} \mathrm{C}\right)$, the tendency to form smectic filaments at the LC-

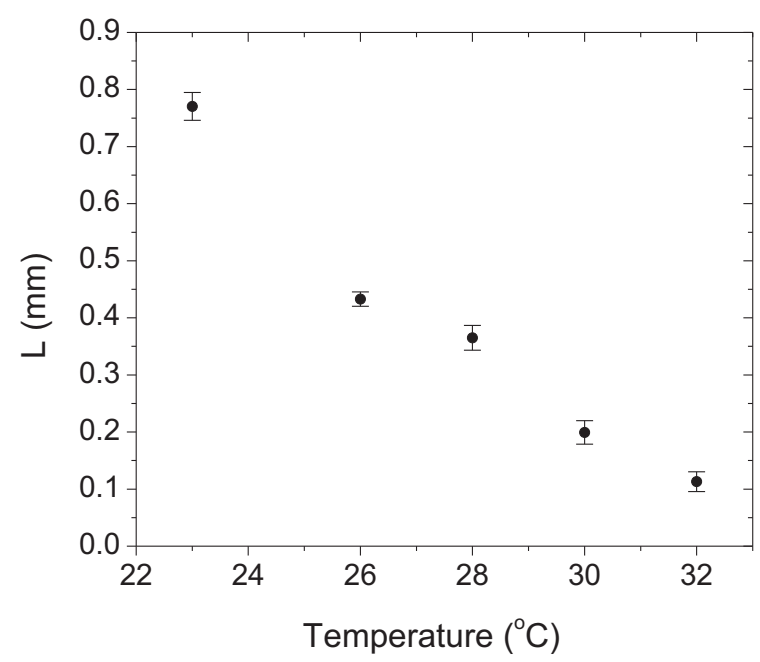

Figure 59: Sandwich method - effect of temperature on growth rates of smectic filaments. Filaments grow faster and for longer time periods as the temperature of the system is decreased. The $L$ values are measured after allowing the filaments to grow for five minutes. 
aqueous interface significantly reduces. Instead, several smectic droplet jets are observed along the LC-aqueous interface. More details about these spontaneous smectic droplet jets are provided in the Section 3.2.4.2. The LC-aqueous interface becomes extremely unstable at temperatures very close to $T_{S m A-N}$. For example, smectic layers break apart continuously and produce droplets. More detailed investigation of the LC-aqueous interface at temperatures near to $T_{S m A-N}$ is highly warranted.

\subsubsection{Effect of the confining walls on the morphology of smectic-A fila- ments}

Fig. 60 shows the effect of the confining walls (or more precisely the effect of the sandwich cell thickness) on the average cross-sectional diameter $(\langle\mathrm{d}\rangle)$ of the $8 \mathrm{CB}$ smectic filaments. The concentration of CTAB and the temperature of the system are maintained at a constant of 40 $\mathrm{mM}$ and $23^{\circ} \mathrm{C}$ respectively. Mylar spacers of different thicknesses are used to study the effect of glass slides on the values of $\langle\mathrm{d}\rangle$. Glass slides are cleaned using plasma cleaner and they are not treated with any surface coupling agents. The influence of the confining walls on the morphology of smectic filaments decreases with increasing spacers' thickness. Predictably, confining walls influence the morphology of lyotropic myelin figures similarly [118]. It is

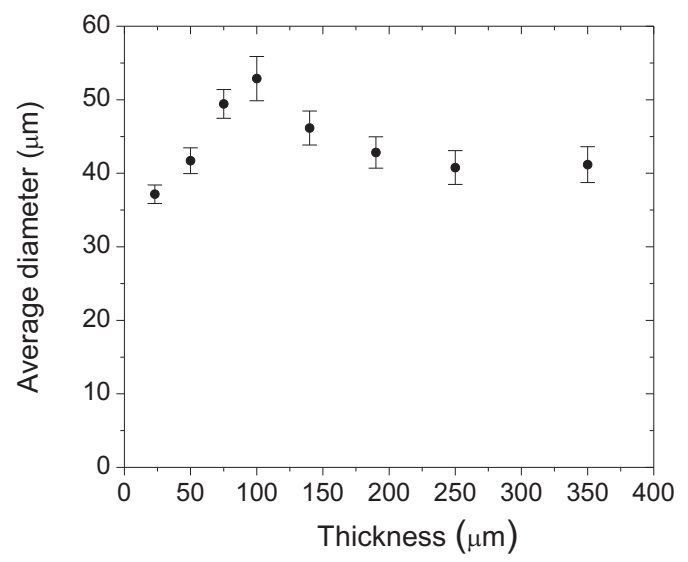

Figure 60: Sandwich method - effect of confining walls on the morphology of smectic filaments. Experiments are conducted with $8 \mathrm{CB}$ and a CTAB $40 \mathrm{mM}$ solution. Filaments increasingly do not feel the presence of confining walls as the spacers' thickness increases. The effect of confining walls on the growth rates of filaments is not yet investigated.

important to note that there is no evidence for the direct influence of the confining walls on the mechanism of filament formation itself. However, if the glass slides are treated with any agent which promotes the ordering in the original liquid crystal sandwich droplet, then the formation of filaments at the LC-aqueous interface becomes unlikely.

\subsubsection{Growth rates of smectic-A filaments: Surfactant chain length de- pendence}

Experiments are carried out with four surfactants from a homologous series of $\mathrm{n}$ - alkyltrimethylammonium bromides $\left(C_{n}\right.$ TAB where $\mathrm{n}=10,12,14$ and 16). Fig. 61 shows the effect of surfactant tail length on the growth of $8 \mathrm{CB}$ smectic-A filaments. Surfactant concentration, temperature and the spacers' thickness are kept constant at $20 \mathrm{mM}, 23^{\circ} \mathrm{C}$ and $100 \mu \mathrm{m}$ respectively. Snapshots of the $8 \mathrm{CB}-C_{n}$ TAB $20 \mathrm{mM}$ systems are taken ten minutes after the initial contact between the $8 \mathrm{CB}$ sandwich droplet and an aqueous $C_{n} \mathrm{TAB}$ micellar solution. 
The width $L$ of the filament-filled region is calculated as in Section 3.2.3.2. The average length of the filament $(L)$ increases and the average cross-sectional diameter of the filament decreases with the increasing chain length of the surfactant. This is due to the increased effectiveness of the higher homologues in reducing the interfacial tension values. The addition of a co-surfactant to the $8 \mathrm{CB}-C_{n} \mathrm{TAB}$ systems significantly increases the interfacial activity (i.e., the cross-sectional diameter of the filaments decreases and the number density of the filaments significantly increases.).

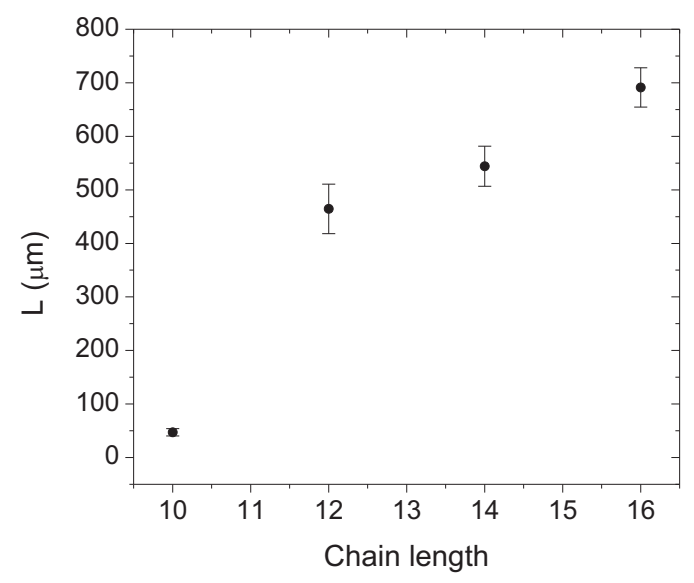

Figure 61: Sandwich method - effect of surfactant chain length on the growth rates of smecticA filaments. The $L$ values are measured after allowing the filaments to grow for ten minutes. At the investigated concentration $(20 \mathrm{mM})$, filaments grow much faster with increasing chain length of the surfactant. However, this type of behavior is unlikely to be observed at very high concentrations. But the LC-aqueous interfacial area is always higher for higher homologues at any given concentration and time of observation.

\subsubsection{Capillary method}

It is impossible to obtain stable filaments through the sandwich method. As shown in Fig. 62, freely floating filaments are only transient structures and they either transform into discs or smectic droplets. Smectic discs may also further transform into vesicles. If there are enough active micelles available in the aqueous CTAB solution, smectic-A liquid crystal emulsion containing all the above mentioned transient structures eventually dissolves completely in the aqueous micellar solution. For example, up to $\sim 3 \mathrm{wt} \%$ of $8 \mathrm{CB}$ produces a clear solution with a TTAB $25 \mathrm{wt} \%$ solution (i.e., all $8 \mathrm{CB}$ molecules are transferred into the TTAB micelles in the aqueous phase). It is important to find a way to stabilize the filaments for any possible practical applications. Stable filaments can be achieved by using the capillary method.

The details of this experimental method are provided in Section 3.2.2. This method provides a way to study the stability and the growth of single filaments. Circular capillaries must be avoided whereas square and rectangular capillaries are best suited for this work. Circular geometry (capillaries with constant diameters or diverging diameters) induce unwanted surface effects on the smectic liquid crystals as shown in Fig. 62(g). As we investigated the properties of strongly confined single filaments only, we used capillaries with square cross-sections exclusively. As shown in Fig. 62(h), square geometry cannot make smectic liquid crystal focal conical defect-free unlike circular geometry. Thus, square capillaries provide an excellent opportunity to study the effect of the ionic surfactant solutions on the disordered smectic liquid crystals. 


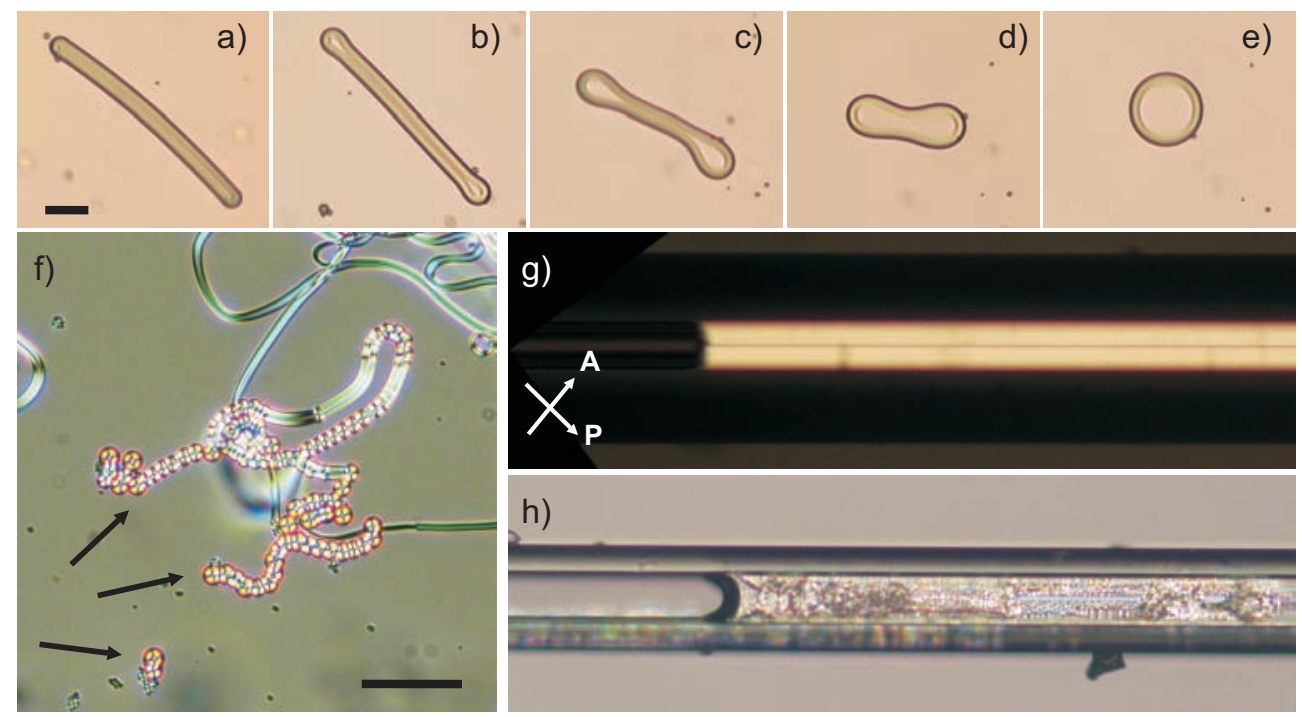

Figure 62: Sandwich method - instabilities in smectic-A filaments. (a-e) A freely floating thick $8 \mathrm{CB}$ smectic-A filament in CTAB $20 \mathrm{mM}$ aqueous solution. These images show the transformation of a filament into a disc. It takes a few minutes to complete the process. Scale bar in image 'a' represents $20 \mu \mathrm{m}$. (f) Thin filaments often break into small droplets instead of forming discs. Black arrows indicate the regions of interest. Scale bar: $50 \mu \mathrm{m}$. (g) $8 \mathrm{CB}$ in an empty circular capillary of $50 \mu \mathrm{m}$ inner diameter. Circular capillary itself induces ordering in $8 \mathrm{CB}$. Thus, they are not suitable for our studies. (h) $8 \mathrm{CB}$ in an empty square capillary of $50 \mu \mathrm{m}$ inner width. $8 \mathrm{CB}$ is full of defects even after confinement in the capillary for a long time. This makes square geometry ideal for our experiments. Images ' $f$ ' and 'g' are taken under slightly uncrossed polarizers.

\subsubsection{Confined single filaments: Effect of the capillary dimensions and the surfactant concentration}

Formation of a single filament in a square capillary (of a given inner edge length) unsurprisingly depends upon the CTAB concentration in the surrounding aqueous solution. Fig. 63(a-c) shows the effect of the capillary edge length and the CTAB concentration on the formation, growth and stability of the single filaments. Fig. 63(a) shows the effect of the CTAB concentration on the growth behavior of an $8 \mathrm{CB}$ filament in a $50 \mu \mathrm{m}$ capillary. It is evidently clear that the initial growth rate of the singe filaments decrease with increasing CTAB concentration. But equilibrium lengths of the filaments increase with increasing CTAB concentration (i.e., despite the non-trivial growth kinetics of the filaments, the equilibrium LC-aqueous interfacial area increases monotonously with increasing CTAB concentration). Another important observation is that $8 \mathrm{CB}$ is highly unlikely to form single filaments in a 50 $\mu \mathrm{m}$ capillary for CTAB concentrations greater than or equal to $20 \mathrm{mM}$. This can be easily understood if we consider the fact that the average cross-sectional diameter of filaments (sandwich method, see Fig. 58(c)) decreases with increasing CTAB concentration. A simple experiment with a capillary of a smaller edge length $(35 \mu \mathrm{m})$ confirms this. Single filaments are produced at much higher CTAB concentrations $(<60 \mathrm{mM})$ in $35 \mu \mathrm{m}$ capillaries, which suggests that the probability of the formation of a single filament decreases with increasing CTAB concentration.

Even though single filaments form inside a capillary, they do not always stay stable indefinitely. There exists a threshold CTAB concentration for a given inner edge length of the capillary below which filaments are unstable. This threshold CTAB concentration seems to be increasing with decreasing capillary dimensions (Fig. 63(c)). Below threshold CTAB 
a)
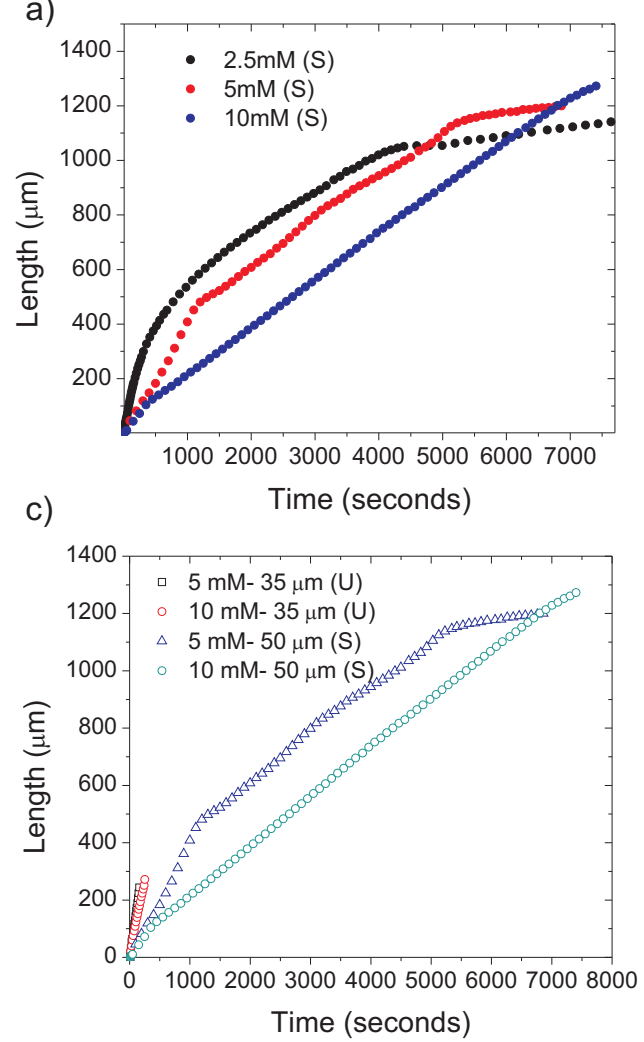

b)
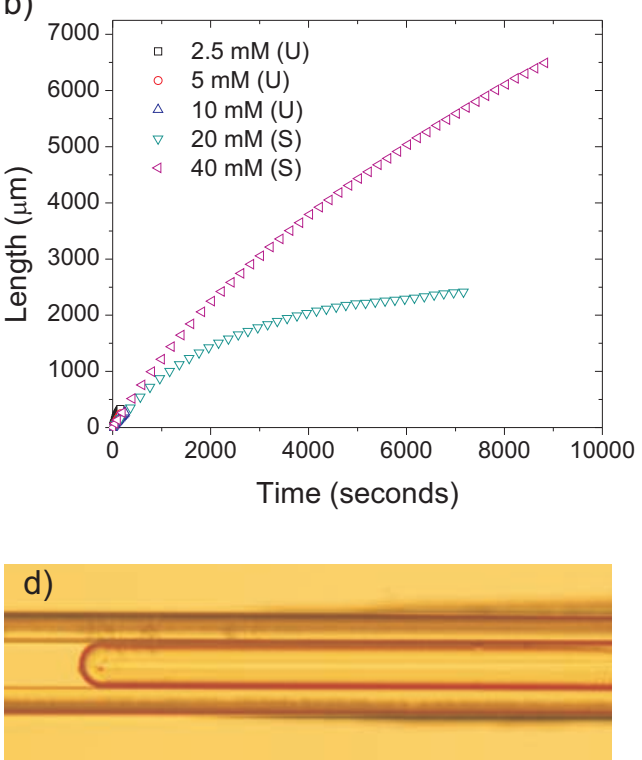

e)

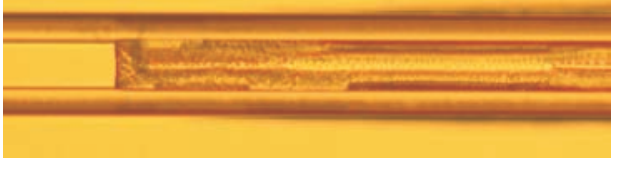

Figure 63: Capillary method - growth behavior of smectic-A filaments in different sizes of capillaries. 'S' and ' $U$ ' characters in the legends of the graphs represent the stable and unstable filaments respectively. (a) An 8CB filament's growth behavior in a $50 \mu \mathrm{m}$ square capillary. Initial growth rate is maximum for lowest investigated CTAB concentration but the length of the filament at equilibrium is minimum at this concentration. A stable filament is difficult to achieve for a concentration below $2.5 \mathrm{mM}$ (The threshold CTAB concentration) in a $50 \mu \mathrm{m}$ capillary. (b) An 8CB filament's growth behavior in a $35 \mu \mathrm{m}$ square capillary. The minimum (or threshold) CTAB concentration needed to form stable filaments is increased to $>10 \mathrm{mM}$. (c) A comparison between the stability of filaments in 35 $\mu \mathrm{m}$ and $50 \mu \mathrm{m}$ capillaries. Apparently, more surfactant is needed to stabilize the filaments in capillaries with a smaller inner width. (d) An $8 \mathrm{CB}$ filament in a $35 \mu \mathrm{m}$ capillary just before the onset of instability. (e) The same filament after destabilization. Cylindrical morphology is lost and $8 \mathrm{CB}$ assumes the structure of the capillary (i.e. square cross section).

concentrations, filaments grow for some time before they abruptly stop and lose their ordered structure. Filaments assume a square cross-sectional shape (i.e., filling the total inner crosssectional area of the capillaries) from their pre-destabilization circular cross-sectional shape (Fig. 63(d-e)). Above the threshold concentrations, filaments stay stable indefinitely after reaching their equilibrium size.

\subsubsection{Smectic-A filaments' growth: Comparison between sandwich and capillary methods}

Fig. 64 compares the growth behavior of smectic-A filaments in a sandwich cell and a square capillary. Surprisingly the initial growth behavior of the smectic-A filaments is almost identical in both methods. But the filament growth slows down much faster in the case of the sandwich method. Due to the lack of confinement, smectic filaments buckle and form complex structures such as coils and curls after an initial growth period of almost 
perfect radial motion (see Section 3.2.2). This means unconfined filaments in a sandwich cell have an extra dimension for motion (angular dimension). But, the capillary method does not allow the filaments to explore more than one dimension (one-dimensional translational motion) which makes them to grow relatively faster than in the case of the sandwich method. Another important difference is that it is impossible to produce stable filaments using the sandwich method, whereas the single filaments in square capillaries can be stable for at least a few months. The reason behind the stability of the filaments in square capillaries is not yet understood.

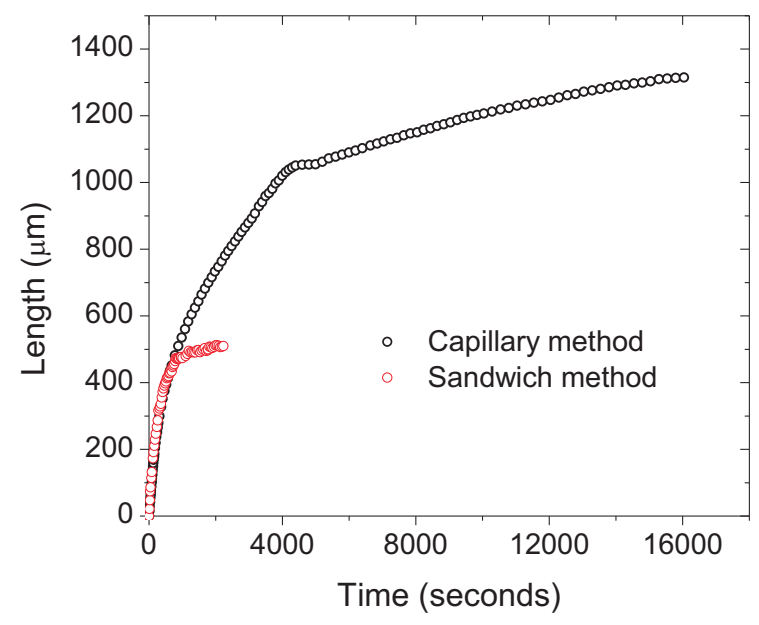

Figure 64: Comparison between sandwich and capillary methods. Experiments are conducted with $8 \mathrm{CB}$ and CTAB $2.5 \mathrm{mM}$ aqueous solutions. Inner edge length of the capillary is 50 $\mu \mathrm{m}$. Thickness of the spacers used for the sandwich experiment is $100 \mu \mathrm{m}$. Initially, there is no effect of geometry on the growth behavior of smectic filaments. Filaments in a sandwich cell eventually destabilize whereas filaments in square capillaries can be stable indefinitely.

\subsubsection{Surface instabilities of smectic filaments: Liquid crystal (LC) ropes}

As shown previously, smectic filaments are only transient structures and they eventually become destabilized through various pathways (except strongly confined single filaments). One particular destabilization pathway may have important biological relevance. As shown in Fig. 65(a), smectic filaments ("parent" filaments) are not stable and thinner smectic filaments ("daughter filaments") may spontaneously start to grow on the surface of the parent filaments. Daughter filaments are pure liquid crystal filaments (just like parent filaments) with no traces of surrounding aqueous solution. This means the total combined volume of parent and daughter filaments is always conserved (Fig. 65(c-f)). The diameter of the daughter filaments can be quite small (of the order of $1 \mu \mathrm{m}$ ) and their growth appears as an ejection process by which a random coil of the daughter filament is formed. We named the daughter filaments also as liquid crystal ropes. Buckling instabilities of the LC ropes which lead to the formation of several beautiful structures are discussed in detail in the following sections. Long daughter filaments eventually decompose into smaller fragments, which can adopt different shapes such as short tubes, small droplets, or vesicle-like structures.

The destabilization of the parent filaments through the generation of daughter filaments is also not yet reported for lyotropic systems. Several studies were conducted previously to investigate the effect of salt or surfactants in the aqueous medium on the lyotropic myelin 

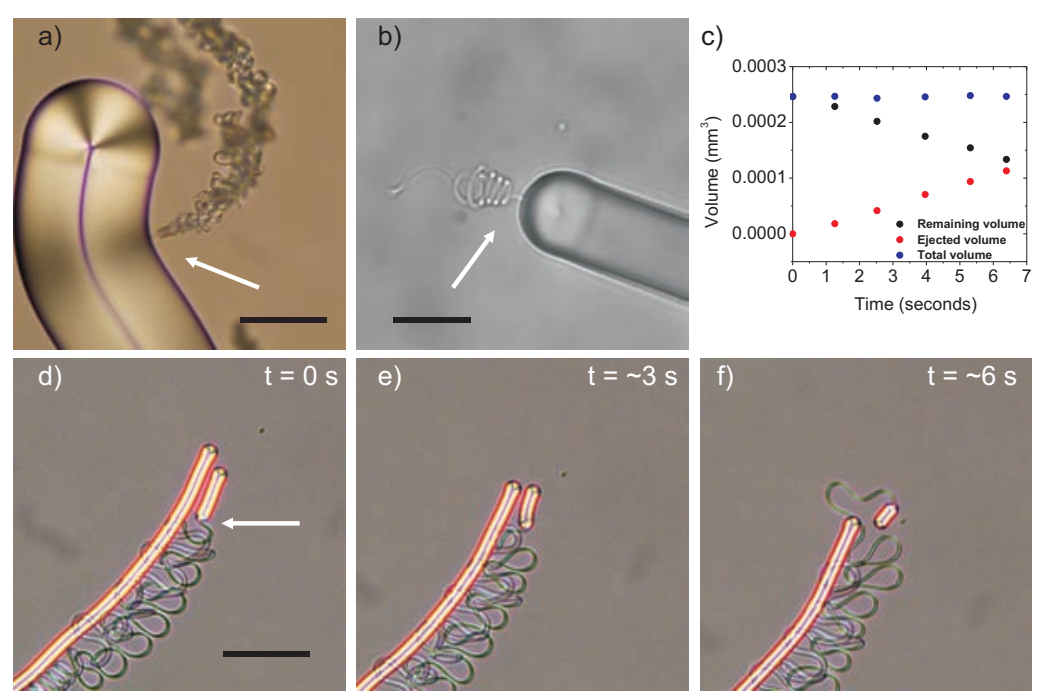

Figure 65: LC ropes - surface instability on thermotropic and lyotropic filaments. White arrows indicate the regions of interest. (a) Optical micrograph of a 12CB filament spontaneously ejecting a daughter filament. Concentration of $\mathrm{CTAB}=280 \mathrm{mM}$. Temperature $=$ $50^{\circ} \mathrm{C}$. Scale bar: $50 \mu \mathrm{m}$. Point of ejection on the surface of the filament (indicated by a white arrow) is not stationary. It can freely move upon the surface of the filament. (b) $L_{\alpha^{-}}$ Phosphatidylcholine in CTAB $200 \mathrm{mM}$ solution at $23^{\circ} \mathrm{C}$. Scale bar: $20 \mu \mathrm{m}$. (c-f) Images d-f provide the snapshots of an ejecting smectic-A filament taken at different times. The conservation of volume is observed throughout the ejection process (image c). This indicates that LC ropes are devoid of any surrounding aqueous solution. Scale bar in image 'd' represents $100 \mu \mathrm{m}$. Images a and d-f are taken under slightly uncrossed polarizers.

figures formed by lipid-water systems $[121,135,168]$, but none of the studies investigated the destabilization pathways of the lyotropic myelin figures. Our preliminary observations show that lyotropic myelin figures also destabilize through the formation of daughter filaments when they are brought into contact with an aqueous CTAB solution or a brine solution instead of pure water (Fig. 65(b)).

Thus, it seems like this process may provide key information about myelin sheath destabilization (demyelination) [157]. The myelin sheath, a fatty insulatory layer around the nerve fibers, enables messages to be transmitted from the brain to anywhere in the body. It is composed of alternating layers of lipids and water, with proteins acting as the binding agents to keep this layered structure intact. Loss of the myelin sheath causes several neurological disorders. The myelin sheath is surrounded by extra-cellular fluid (ECF) which contains several ions and proteins. It has been observed that the myelin sheath gets severely destabilized when its composition of lipids and proteins changes [169-171]. The exact routes of destabilization or fragmentation are yet to be discovered. Thus, a detailed investigation of the surface instabilities of lyotropic and thermotropic filaments is necessary. Our following experimental results with thermotropic smectic filaments do suggest that myelin sheath stability may depend on the ionic strength of the ECF and the interfacial tension between the myelin sheath and the ECF.

\subsubsection{LC ropes: Why do they form on the surface of smectic filaments?}

It is argued in the earlier sections that smectic filaments form at the LC-aqueous interface as a result of competition between the LC bulk defects' energy and interfacial energy. Thus, 

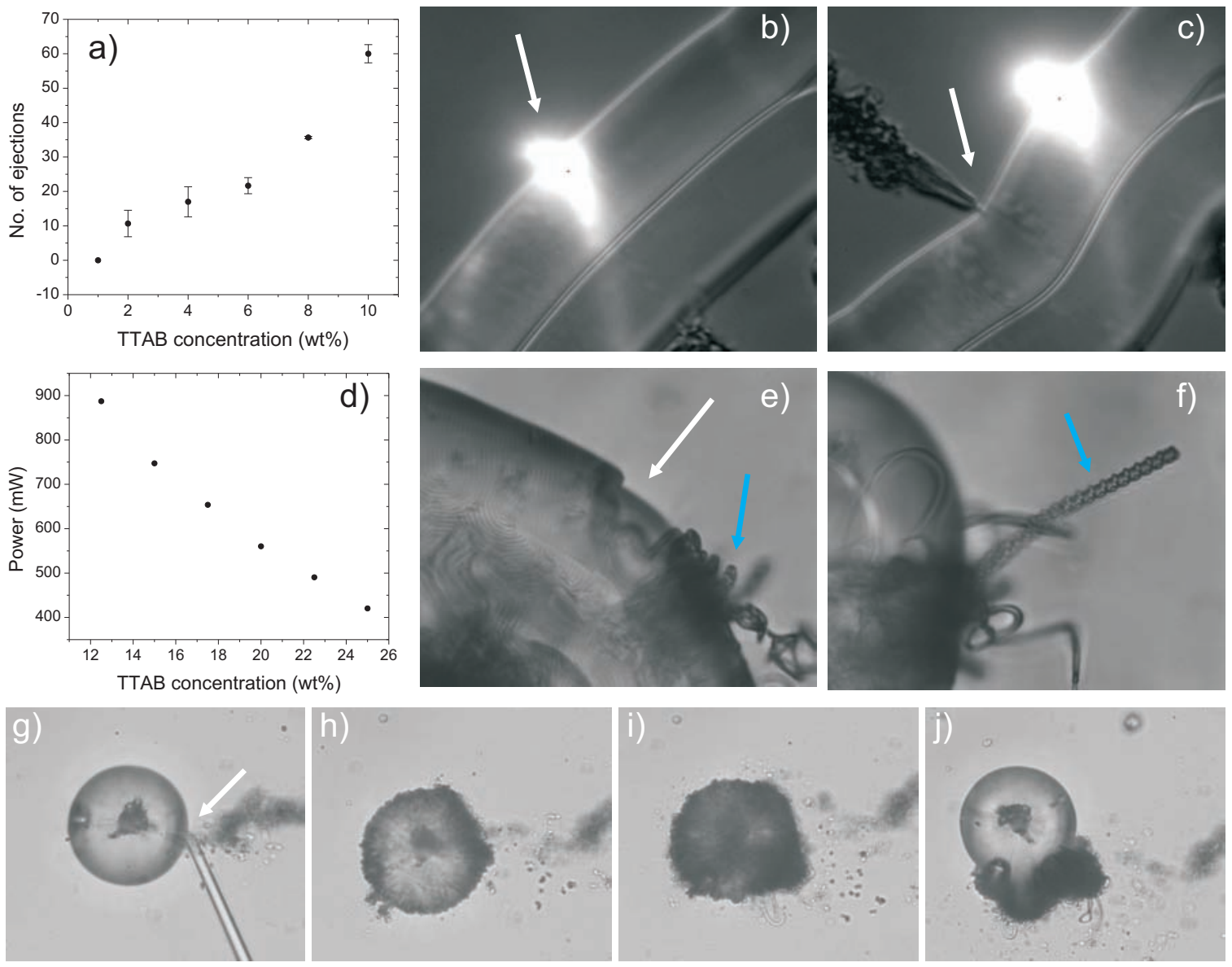

Figure 66: Destabilization of the LC-aqueous interface using different methods. (a) The number of spontaneous ejections observed along the $8 \mathrm{CB}$-aqueous interface is plotted against the concentration of TTAB in the aqueous solution. (b-c) Laser tweezers method: Infrared laser tweezers can be used to heat the surface smectic layers and produce LC ropes. But for improved heat distribution, $0.01 \mathrm{wt} \%$ Nile red is added to $8 \mathrm{CB}$. A continuous wave (514 nm) $\mathrm{Ar}^{+}$laser is used to heat the smectic layers locally as shown in Figure b. As indicated by white arrows, an LC rope is generated exactly at the point of exposure to the laser beam. Ignore the bright white spot in the image (c) as it represents the new position of the $\mathrm{Ar}^{+}$laser. Horizontal width of the images is $143 \mu \mathrm{m}$. (d) The minimum power of infrared laser $(1064 \mathrm{~nm})$ needed for destabilizing the $8 \mathrm{CB}$ surface layers is plotted against the concentration of TTAB in an aqueous solution. (e) If we use infrared laser tweezers, they will heat up a large LC surface area which results in the formation of a numerous number of filaments instead of just one. Inner smectic layers are clearly visible as well as with damaged surface layers (indicated by the white arrow). Some of the produced filaments at the $8 \mathrm{CB}$-aqueous interface can further self-assemble to produce complex structures. The blue arrow indicates one such case where we observe a double stranded helix formed by two filaments. (f) A double stranded helix grows very rapidly ( $<1$ second per pitch). Horizontal width of both 'e' and ' $\mathrm{f}$ ' images is $83.75 \mu \mathrm{m}$. (g-j) Fiber method: An 8CB-aqueous interface can also be destabilized by scratching the surface with a fiber. The white arrow in image 'g' indicates the position of scratching. Damaged surface layers are cast off by the smectic droplet (but they do not necessarily get detached completely from the droplet). This process is reminiscent of the moulting phenomenon observed in Biology. Initial size of the droplet is $29 \mu \mathrm{m}$. 
it should be quite surprising to see the formation of LC ropes on the surfaces of perfectly ordered smectic filaments. But our following results show that LC ropes do form due to the same reason that smectic filaments form in the first place.

Fig. 66(a) shows the dependence of 8CB-aqueous interface (i.e., smectic-A filaments) stability on the TTAB concentration in the aqueous medium. The temperature of the sandwich cells and the spacers' thickness are kept constant at $23^{\circ} \mathrm{C}$ and $100 \mu \mathrm{m}$ respectively. $1 \mathrm{mg}$ of $8 \mathrm{CB}$ is used as an original sandwich droplet for each experiment. This means the initial exposed free surface area of $8 \mathrm{CB}$ to the TTAB solutions is $1.13 \mathrm{~mm}^{2}$. The total observation period for counting the number of spontaneous ejections of LC ropes from the surfaces of smectic filaments at the $8 \mathrm{CB}$-aqueous interface is chosen as 10 minutes. The ejection of LC ropes is not a constant process over time and the number of ejections from the LC-aqueous interface (i.e. from the surfaces of the smectic-A filaments) decreases almost exponentially over time.

It is clear from Fig. 66(a) that the $8 \mathrm{CB}$-aqueous interface (more precisely, the smecticA filaments at the $8 \mathrm{CB}$-aqueous interface) becomes increasingly susceptible to ejecting LC ropes out into the aqueous medium with increasing TTAB concentration. Similarly, increasing susceptibility of the $8 \mathrm{CB}$-aqueous interface is observed by increasing salt concentration in the aqueous medium instead of TTAB concentration. The addition of small amounts of co-surfactants also significantly increases the number of ropes ejected from the LC-aqueous interface per unit area per unit time. These observations show that interfacial tension plays a major role in destabilizing the LC-aqueous interface. LC ropes can also be initiated force-

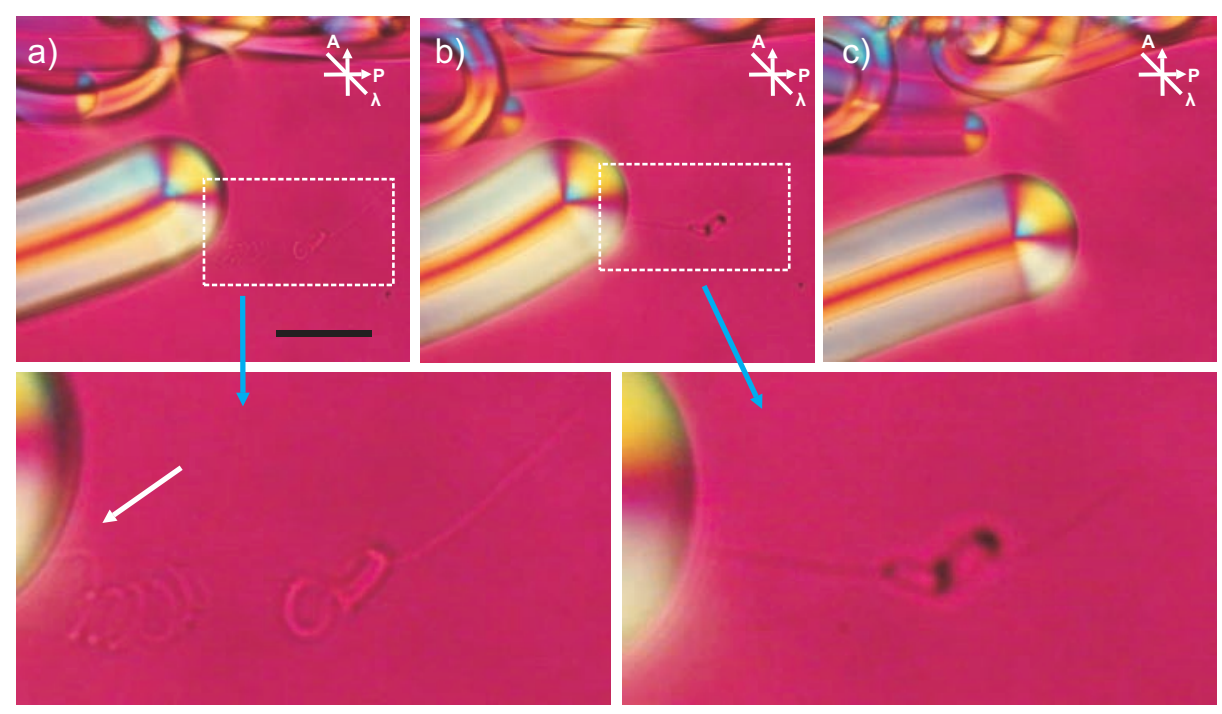

Figure 67: Self-healing lyotropic myelin figure. $L_{\alpha^{-}}$Phosphatidylcholine in CTAB $200 \mathrm{mM}$ solution at $23^{\circ} \mathrm{C}$. Optical tweezers are used to disturb the surfaces of myelin figures. Enlarged sections of the images (a) and (b) are shown in the bottom row. (a) The white arrow in the enlarged section of the image (a) indicates the leaking position on the surface of a myelin figure. The leaking of material in the form of an LC rope happens exactly at the point of exposure to the optical tweezers. Scale bar: $40 \mu \mathrm{m}$. (b) If we wait for a few minutes, the ejection process stops and the ejected LC rope starts to retract into the parent structure. (c) The ejected LC rope is completely retracted into the parent structure. The reintegration process of the LC rope into the parent structure is so perfect that later it is impossible to find the spot from where it was ejected. It is important to note that this self-healing process is only possible if the ejected LC rope retains its structure and does not undergo any structural transformation (such as into the vesicular structure) during this entire process. 
fully by creating defects on the surface of parent filaments, either by using short heat pulses generated by optical tweezers or by mechanically disturbing their surfaces with microfibers (Fig. 66(b-j)). Experimental observations show that the threshold power (of the incident infrared beam) required for producing LC ropes on the surfaces of smectic filaments decreases with increasing TTAB concentrations (Fig. 66(d)). This means that even the slightest disturbance on the surface of a smectic filament in a highly concentrated TTAB solution results in the formation of one or more LC ropes at the point of disturbance. Combining the results of both spontaneous and artificially-forced ejections of LC ropes, it is fair to conclude that daughter filaments are also a result of the interplay between bulk and interfacial energies.

Another simple experiment with lyotropic myelins further explains the dynamics of the LC ropes. As shown in Fig. 67(a), an LC rope is ejected from a lyotropic myelin figure after exposing it to a short heat pulse produced by an infrared laser beam. The supplied energy must have been enough to produce more interfacial area in the form of an LC rope. However, the destabilized myelin figure heals itself as the heat energy is dissipated into the surroundings over time (Fig. 67(b-c)). This observation further supports our arguments about the instability's origin.

\subsubsection{Temperature dependence of the spontaneous ejections of LC ropes}

Fig. 68 shows the temperature dependence of the spontaneous ejections of LC ropes from an $8 \mathrm{CB}$-aqueous interface (more precisely, from the surfaces of the smectic-A filaments at an $8 \mathrm{CB}$-aqueous interface). The concentration of TTAB, the spacers' thickness and the weight of the $8 \mathrm{CB}$ sandwich droplet are fixed at $6 \mathrm{wt} \%, 100 \mu \mathrm{m}$ and $1 \mathrm{mg}$ respectively. Even though Fig. 68 suggests a complex relationship between ejections and temperature, the number of ejections per unit area becomes almost zero as we approach smectic-A to nematic phase transition. Another important point to take note of is that the LC-aqueous interface

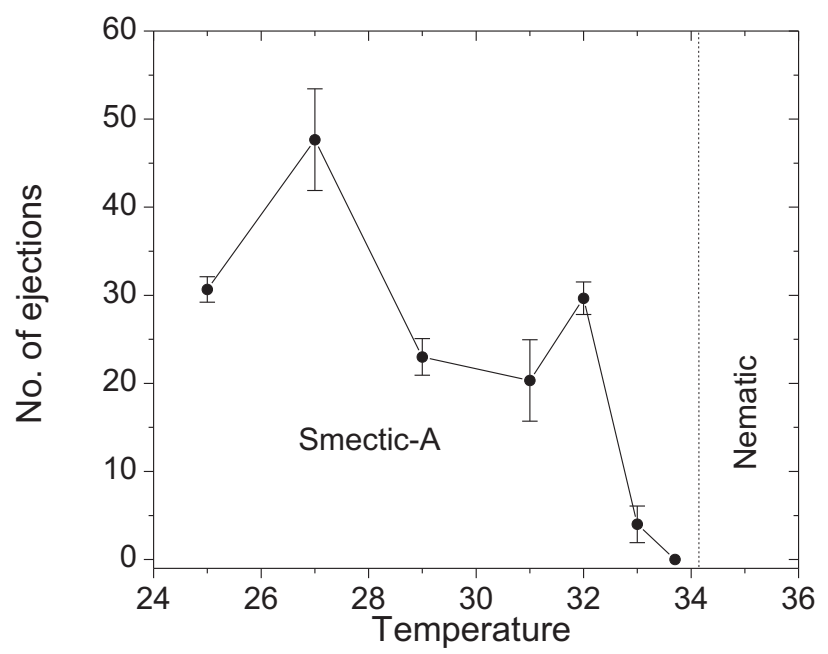

Figure 68: Sandwich method - effect of temperature on the stability of 8CB-aqueous interface. The number of ejections observed along the $8 \mathrm{CB}-\mathrm{TTAB} 6 \mathrm{wt} \%$ solution interface is plotted against the temperature of the system. Please note that the temperatures on the abscissa are the values shown by the heating device. At the Smectic-A - Nematic transition temperature, smectic layers at the interface directly break into tiny nematic droplets (this does not happen at an 8CB-pure water interface). It is important to note that we do not differentiate between rope ejections and droplet ejections while counting the number of ejections. 
leaks smectic droplets instead of LC ropes with increasing temperature. In the case of the investigated 8CB-TTAB $6 \mathrm{wt} \%$ system, the probability of the ejection of droplets becomes significantly higher than the ejection of LC ropes above $31^{\circ} \mathrm{C}$. Similarly, the probability of ejecting droplets instead of LC ropes increases with increasing TTAB concentration.

\subsubsection{LC ropes: Liquid rope coiling effect}

The liquid rope coiling effect is observed when a thin stream of viscous fluid is poured onto a surface [172-175]. Instead of approaching the surface vertically, viscous fluid streams form beautiful ordered structures. For example, a thin stream of honey builds a spectacular helical structure of radius $R$ that resembles a pile of coiled rope. The liquid rope coiling phenomenon is fundamentally a buckling instability in which a fluid stream is subjected to an axial compressive stress. Under axial compressive stress, thin viscous fluid streams become unstable to deformation by bending. Coiling occurs in three distinct dynamical regimes (viscous, gravitational, and inertial) depending on how the viscous forces in the helical coil are balanced. The dynamical regime in which coiling takes place is determined by the magnitudes of the viscous $\left(F_{V}\right)$, gravitational $\left(F_{G}\right)$ and inertial $\left(F_{I}\right)$ forces per unit length of the coil.

$$
\begin{gathered}
F_{V} \sim \rho v a^{4} U R^{-4}, \\
F_{G} \sim \rho g a^{2}, \\
F_{I} \sim \rho a^{2} U^{2} R^{-1}, \\
U \equiv \frac{Q}{\pi a^{2}},
\end{gathered}
$$

where $\rho$ and $v$ are the density and the viscosity of the viscous fluid respectively. ' $a$ ' represents the radius of the liquid rope, which is assumed to be constant. This assumption holds true only in the current LC ropes' case as the effect of gravity on them is highly insignificant $\left(F_{G} \sim 0\right)$. $Q$ represents the volumetric rate of the ejected liquid crystal.

Fig. 69(a) shows the typical helical structure formed by LC ropes. An ejected LC rope generally forms a helical coil of radius $R$ that rotates with an angular coiling frequency $\Omega$ about an axis normal to the filament surface at the point of leaking. The conservation of mass flux requires $U=\mathrm{R} \Omega$. Experiments show that both gravity and inertia are negligible and the net viscous force is zero at any position inside the LC ropes (viscous regime). In this viscous regime, the $\Omega$ is independent of material constants and it depends only on $Q$ and $a$ values.

$$
R \sim L, \quad \Omega \sim \frac{U}{L}
$$

where $L$ is the distance between the point of leaking and the onset point of coiling (Fig. 69(b)). As expected, there is no preferred sense of rotation for LC rope coiling. Interestingly, the sense of rotation flips between clockwise to counter-clockwise randomly. It is not yet known whether it is possible to control the values of $Q$ and $a$. Finding a way to control these parameters is highly desired as it would provide a way to investigate the LC rope coiling effect systematically. Apart from helical structures, folded in-plane micro threads are also occasionally observed as shown in Fig. 69(c). 

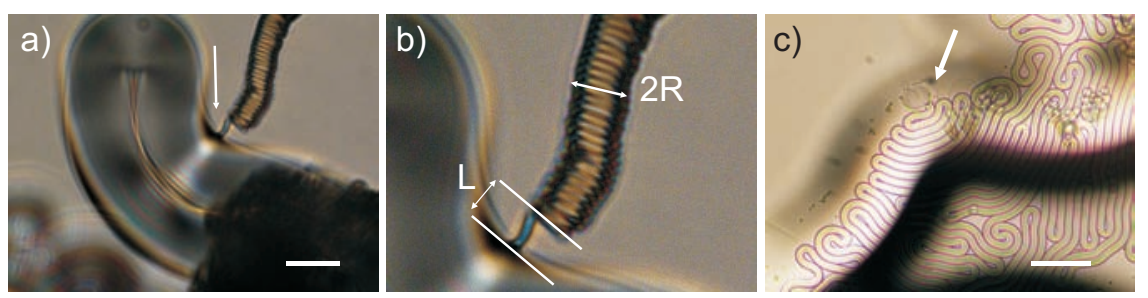

Figure 69: Typical coiling structures observed in LC-aqueous systems. White arrows in images 'a' and 'c' indicate the points of ejection at the LC-aqueous interface. (a) A helical coil is being ejected from a $12 \mathrm{CB}$ filament. The aqueous solution contains $80 \mathrm{mM} \mathrm{CTAB}$ and $0.5 \mathrm{M} \mathrm{NaCl}$. Temperature $=50^{\circ} \mathrm{C}$. Scale bar: $25 \mu \mathrm{m}$. Image is taken under slightly uncrossed polarizers. (b) Magnified version of image 'a' showing the helical coil emanating from the $12 \mathrm{CB}$ filament. (c) If the position of the defect lies very close to the glass slides, folded micro-threads form instead of helical coils due to geometrical constraints. Scale bar: $30 \mu \mathrm{m}$.

\subsubsection{LC rope helical coils: Effect of salt in the aqueous phase}

Due to its charged surface, intra-membrane contacts of an LC rope in a pure CTAB solution are not favorable. LC ropes spontaneously transform their structure into 'bunch of grapes'like structures as shown in Fig. 70. Transition from a liquid rope to a bunch of vesicles is extremely favorable due to the reduction in the interfacial area. The addition of a small amount of salt to the aqueous phase significantly alters the dynamics of the LC ropes. It was already shown earlier that the addition of a salt to the aqueous phase greatly enhances the membrane-membrane attractions. This membrane-membrane attraction causes the ejected LC rope to turn into a compact hollow cylindrical structure (Fig. 69(a-b)). The cylindrical structure further destabilizes and forms a spectacular beads-on-a-string (BOAS) structure in order to achieve a lower energy state (Fig. 71). The three dimensional structure of the BOAS morphology can be easily visualized through the red wave plate images (Fig. 71(gi)). The BOAS structures appear yellow and blue in color when they are positioned parallel and perpendicular to the red wave plate respectively. For a positive birefringent uniaxial
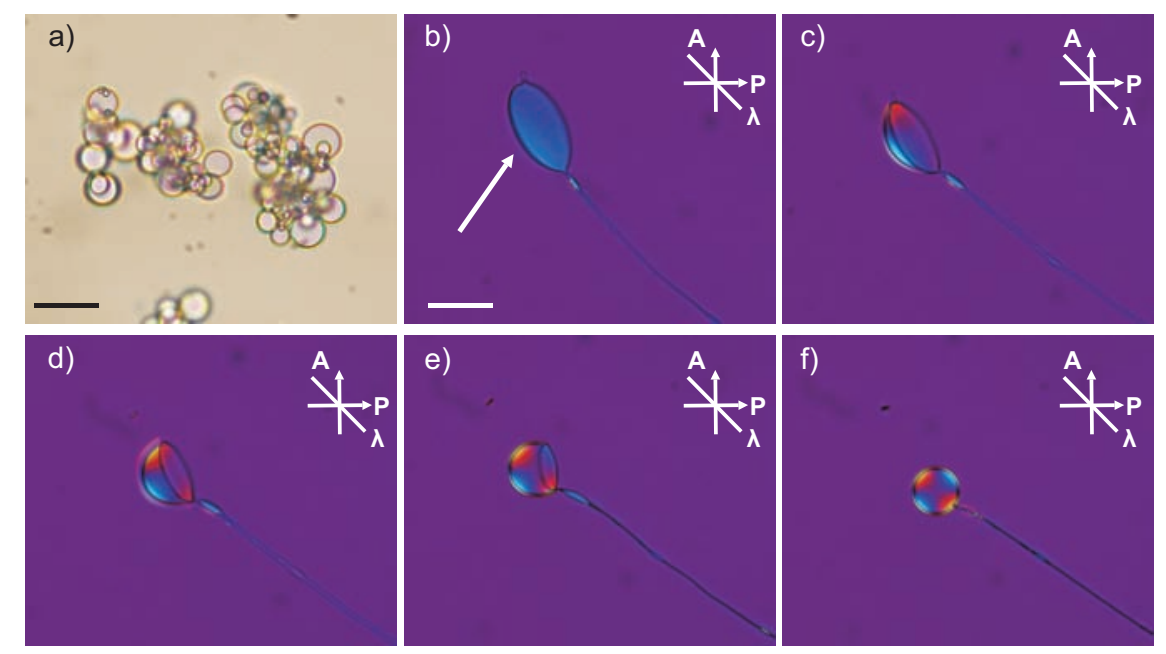

Figure 70: $L C$ rope to vesicle transformation. (a) $8 \mathrm{CB}$ in CTAB $40 \mathrm{mM}$ solution at $23^{\circ} \mathrm{C}$. An ejected LC rope transforms into interconnected 'bunch of grapes'-like vesicles. Scale bar: $25 \mu \mathrm{m}$. (b-f) These images provide snapshots of the rope to vesicle transformation. A few regions in an LC rope expand and form disc-like structures. These discs eventually collapse to form vesicles. Scale bar in image 'b' represents $25 \mu \mathrm{m}$. 

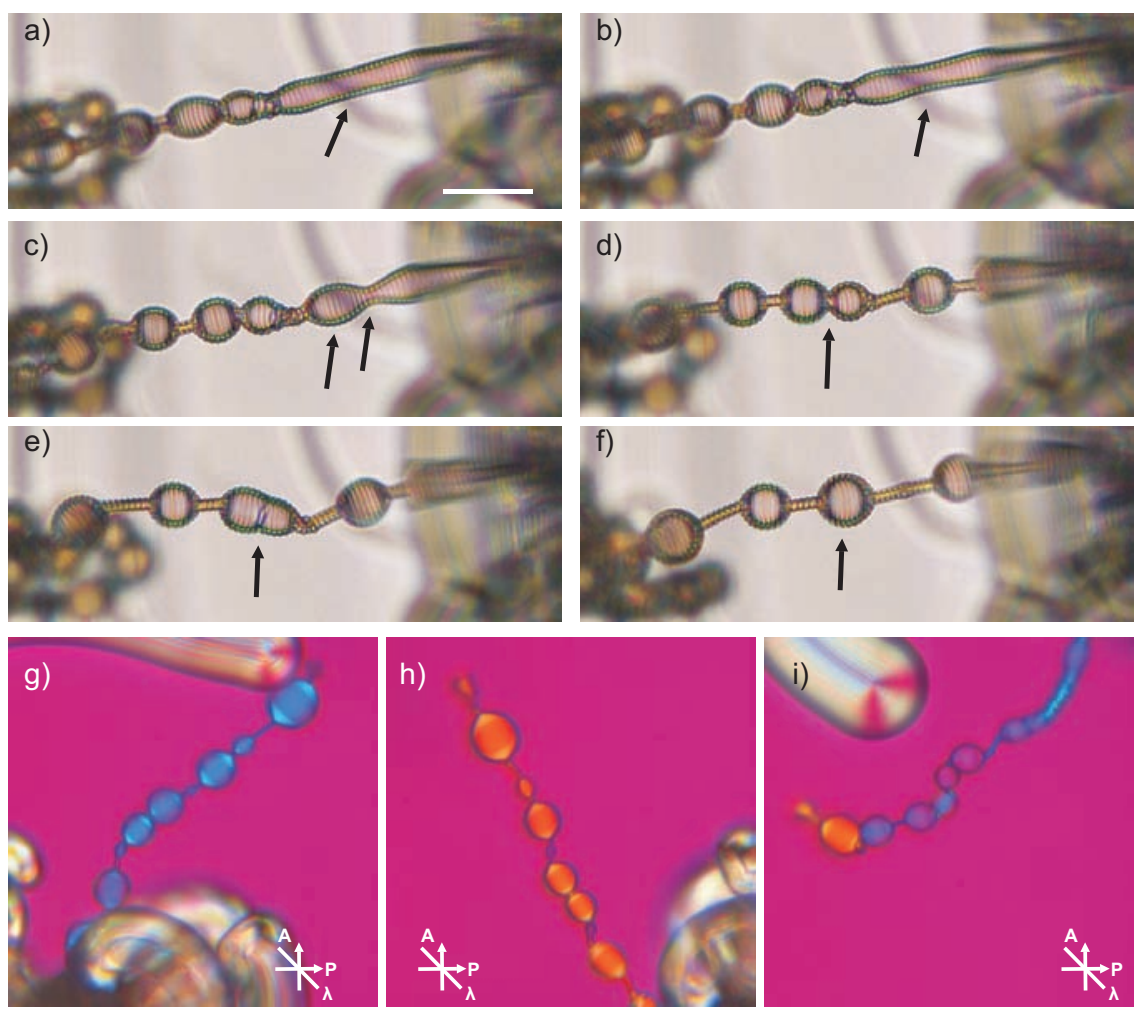

Figure 71: BOAS structure - effect of salt on the LC rope helical coil structure. The LC mixture contains $85 \mathrm{wt} \% 12 \mathrm{CB}$ and $15 \mathrm{wt} \% \mathrm{CB} 15$. Pure $8 \mathrm{CB}$ and $12 \mathrm{CB}$ can also produce these structures. Aqueous solution contains $80 \mathrm{mM} \mathrm{CTAB}$ and $0.5 \mathrm{M} \mathrm{NaCl}$. (a-b) Black arrows indicate the onset of undulation whose wavelength is much higher than the circumference of the circular cross-section. Scale bar in image 'a' represents $50 \mu \mathrm{m}$. (c) Black arrows show the formation of crests and troughs. (d-f) Transformation into a bead structure is complete but two of its neighbors start to fuse into each other (as indicated by black arrows). (gi) Polarizing microscopy images of a completely transformed and a partially transformed BOAS structure. Horizontal width of all three images is $110 \mu \mathrm{m}$.

material ( $8 \mathrm{CB}$ or $12 \mathrm{CB}$ ), this combination of colors suggests a hollow 'single layer' LC rope structure with a variable cross-sectional area.

The transformation from a hollow cylindrical structure into a BOAS structure is a result of classic Rayleigh-Plateau instability. This transformation is driven by interfacial tension which results in the net decrease of the LC rope-aqueous interfacial area. For wavelengths larger than a cylinder's circumference, axisymmetric undulations of the cylinder can reduce its surface area while maintaining constant volume. For perturbations obeying this condition, the internal pressure in the troughs is higher than that in the crests, thereby leading to an instability. For wavelengths shorter than the cylinder's circumference, the pressure in the troughs is smaller than that in the crests and therefore the undulations die down.

\subsubsection{UV photo-polymerization of smectic filaments}

Freely floating smectic filaments are thermodynamically unstable and this makes it difficult to use these filaments for any possible practical applications. It was shown earlier that filaments are stable indefinitely if they are strongly confined in capillaries. Even though the confined filaments are highly stable, confinement severely restricts the scope of their utility. There is a possible way to circumvent this problem and produce free floating stable smectic filaments. This can be achieved by using the UV photo-polymerization method through 
which freely floating smectic filaments can be immobilized in their non-equilibrium states.

Liquid crystal (12CB) is doped with a liquid crystalline acrylate monomer (RM 305), a cross linker (RM 257) and a photo-initiator (Irgacure 907). Predictably, the phase transition temperature of this mixture changes with the dopants' concentration as shown in Fig. 72(a). Irgacure 907 exhibits maximum UV absorbance at $320 \mathrm{~nm}$. RM 305 molecules always align parallel to the liquid crystal molecules due to their rod-like structure. Thus, the resulting polymer network also retains the original alignment of the liquid crystal. Once the polymer network is formed, smectic filaments can be removed from the aqueous solution and used for any purpose. It is important to note that liquid crystal molecules surrounded by the polymer network still retain their liquid crystalline properties. Thus, even polymerized filaments must be maintained at temperatures where liquid crystal molecules are in smectic phase in order to avoid changes in the internal molecular structure.

Fig. 72(b-c) shows a polymerized 12CB sandwich droplet with polymerized smectic filaments at an LC-aqueous interface. The polymerizability of the smectic filaments clearly suggests that all the four components in the sandwich droplet diffuse into the aqueous medium from the bulk of the LC droplet. It is also possible to stabilize smectic filaments with attached LC ropes as shown in Fig. 72(d). Even more complicated structures like the double stranded helix are also retained after polymerization (Fig. 72(e)). Even though this method is successful in producing stable smectic filaments, there is one glaring disadvantage. The surface of the smectic filaments is extremely smooth before polymerization. This enables them to be used for optical applications which are discussed in the following sections. Polymerization of the smectic filaments causes their surface to buckle and become rough. Filaments with rough surfaces are not suitable for optical applications due to huge scattering losses.
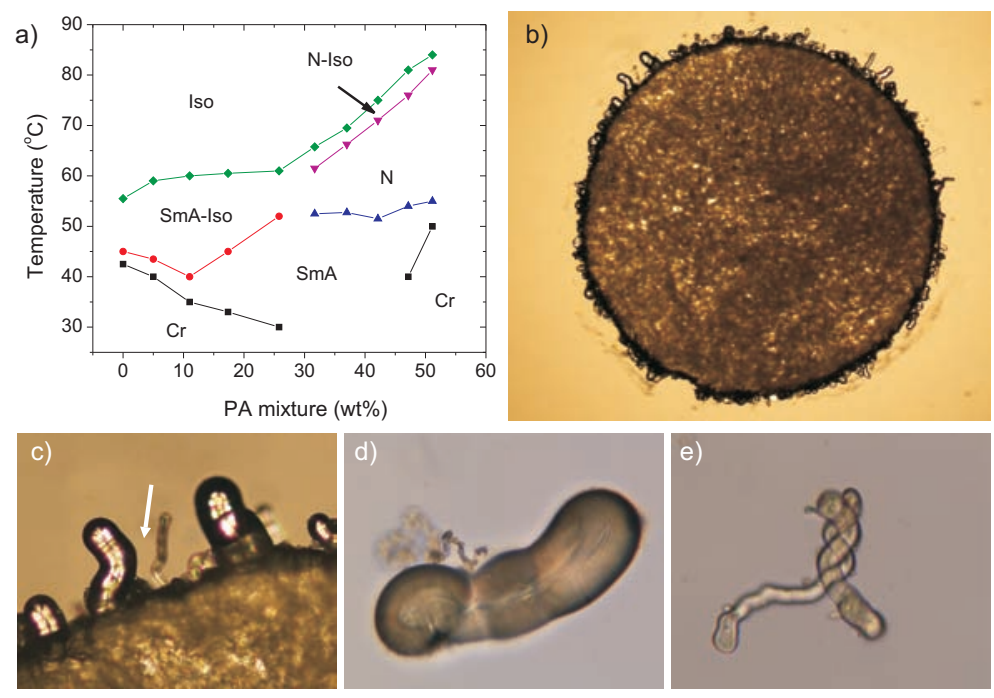

Figure 72: UV photo-polymerization of smectic-A filaments. (a) Two different mixtures are prepared separately. Mixture-1 contains $24 \mathrm{mg}$ of Irgacure 907 and $450 \mathrm{mg}$ 12CB. Mixture-2 contains equal amounts of RM 305 and RM 257. PA mixture wt\% = (Mixture2)*100/(Mixture-1+Mixture-2). N - nematic; SmA - smectic-A; Iso - isotropic; Cr - crystal; $\mathrm{SmA}$ - Iso - coexistence region of smectic and isotropic phases; and N-Iso - coexistence region of nematic and isotropic phases. (b) A polymerized $12 \mathrm{CB}$ droplet with $50 \mathrm{wt} \%$ PA mixture. Horizontal width of the image is $1.7 \mathrm{~mm}$. (c) Magnified version of the previous image showing the polymerized filaments at the $12 \mathrm{CB}$-aqueous interface. Horizontal width of the image is $289 \mu \mathrm{m}$. (d-e) Different structures assumed by filaments are stabilized indefinitely. Horizontal widths of the images 'd' and 'e' are $136 \mu \mathrm{m}$ and $109 \mu \mathrm{m}$ respectively. 


\subsubsection{Self-propelled motion of smectic droplets}

Surface tension gradients drive the motion of nematic and cholesteric droplets with 'no visible material exchange' across the LC-aqueous interface. Self-propelled motion of the nematic and cholesteric droplets is always accompanied by convective flows around the droplets (both inside and outside the droplet, see Section 3.1.8). But self-propelled motion of the smectic droplets is very different from that of the nematic and cholesteric droplets.

$8 \mathrm{CB}$ smectic-A droplets are produced by shaking the mixture of a small amount of $8 \mathrm{CB}$ in an aqueous $0.1 \mathrm{mM} \mathrm{CTAB}$ solution. This method produces radial droplets with radial line defects. As shown in Fig. 73(a-b), this line defect extends from the center to the surface of a radial droplet. There is often a small amount of highly disordered $8 \mathrm{CB}$ at the intersection between the line defect and the surface of the droplet. Smectic droplets move significantly slower than nematic droplets in a given surfactant solution. No internal convection is observed at any stage of the self-propulsion of smectic droplets. As we increase the concentration of surfactant in the surrounding aqueous solution, smectic droplets start to eject tiny ropes or daughter droplets from the disordered $8 \mathrm{CB}$ region existing at the interface, thereby imparting momentum to the droplets (Fig. 73(c-d)). It seems as though the thrust force generated by the ejection of droplets is significantly higher than the surface tension gradients. Thus, self-propelled motion of the smectic droplets is dictated by Newton's third law at high surfactant concentrations (> CTAB 5wt \%).
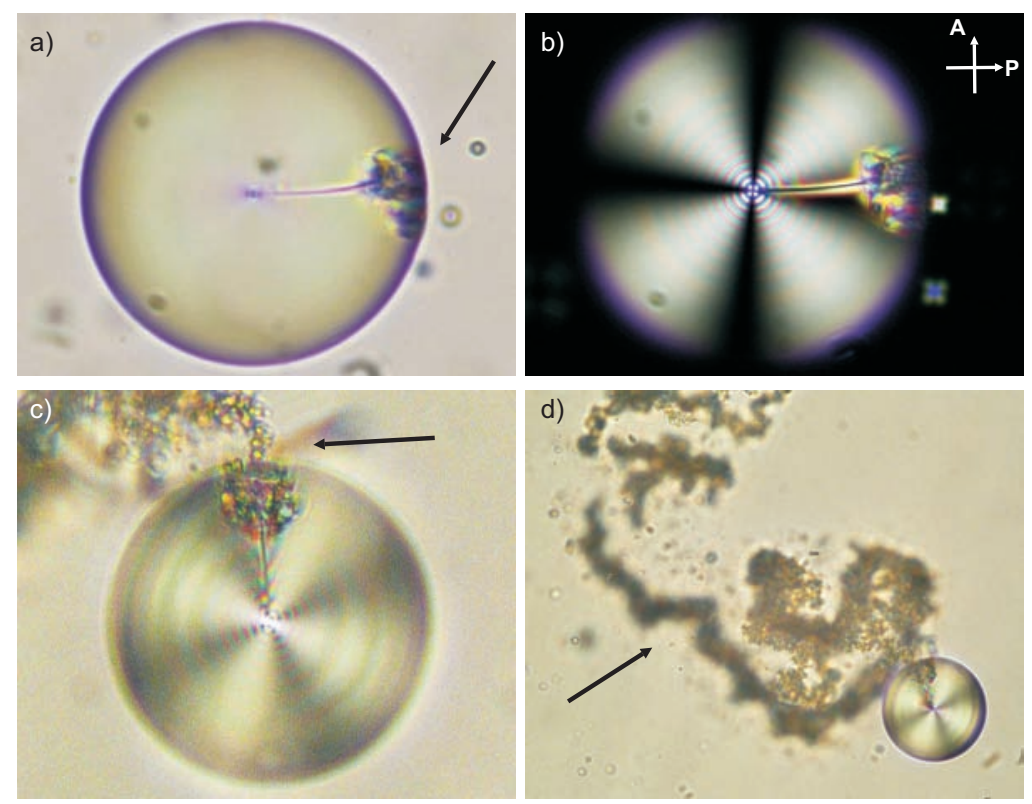

Figure 73: Self-propelling motion of an $8 C B$ smectic- $A$ droplet. (a) 8CB droplets are prepared by dispersing $8 \mathrm{CB}$ in a CTAB $0.1 \mathrm{mM}$ solution in order to avoid the formation of filaments at room temperature. Droplets have no motion in this aqueous solution. This white light image shows an $8 \mathrm{CB}$ droplet with a line defect. The black arrow indicates the disordered $8 \mathrm{CB}$ region existing at the $8 \mathrm{CB}$-aqueous interface. Droplet diameter is 63 $\mu \mathrm{m}$. (b) The same droplet as seen under crossed polarizers. (c) If we transfer the smectic droplets from the CTAB $0.1 \mathrm{mM}$ solution into a CTAB $10 \mathrm{wt} \%$ solution, smectic droplets spontaneously start to propel themselves by ejecting material into the surrounding aqueous solution. This white light image shows a self-propelling $8 \mathrm{CB}$ droplet. It is clearly visible that tiny $8 \mathrm{CB}$ droplets are being ejected from the disordered $8 \mathrm{CB}$ region (as indicated by the black arrow). Droplet diameter is $55 \mu \mathrm{m}$. (d) The black arrow indicates the trail left by the self-propelling droplet. 


\subsubsection{Optical applications of smectic filaments}

The surface tension between two immiscible fluids creates extremely smooth micro-droplets of a spherical shape. Several groups have successfully shown on a laboratory scale that the spherical micro-droplets (both isotropic and liquid crystalline) could be used as optical cavities for tunable resonators, lasers, optical filters, etc [103-105, 176-182]. However, just spherical shapes are not sufficient for the realization of soft photonic circuits. As we know by now, the interplay between surface energy and elastic bulk energy could produce filament-like self-assembled structures. It is important for us to stress that both lyotropic (myelin figures) and thermotropic filament-like structures (nematoids) have been previously reported by several groups [183-189]. However none of these reported systems are suitable for any optical applications. In the case of lyotropic myelin figures, the small refractive index difference between them and their surrounding aqueous environment renders these filaments unsuitable for optical applications. Nematoids are observed during the phase separation between thermotropic LCs and isotropic oils. The main drawback of this system is that it is almost impossible to predict the structures and the positions of these thermotropic filamentlike structures in a phase separated mixture of oils. The current system of LC-water (or any other immiscible fluid) dispersions clearly overcomes these drawbacks.

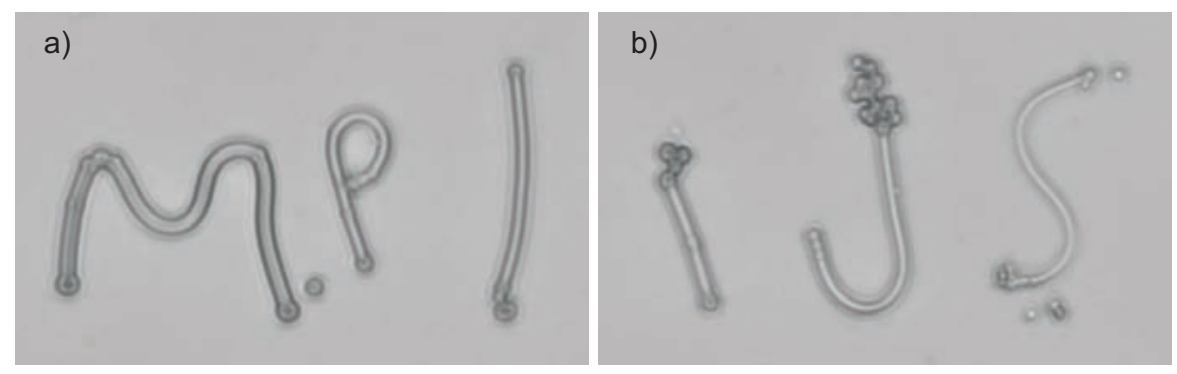

Figure 74: Production of the desired structures by manipulating smectic filaments. Infrared "laser tweezers" (1064 nm) are used to position smectic filaments. These two structures represent the abbreviated forms of the institutes where I did my thesis related-work. (a) MPI: Max Planck Institute. (b) IJS: Institut "Jožef Stefan". Attempts are being made to produce optically useful structures such as rings (by fusing both end-caps of the filaments). Horizontal width of the images is $73 \mu \mathrm{m}$.

We were extremely excited about investigating the photonic properties of thermotropic smectic filaments because of the following reasons: (i) The coaxial organization of smectic layers could give rise to very precise and uniform diameters of the filaments to one molecular length $(2-3 \mathrm{~nm})$. Filaments of various dimensions can be easily produced according to our needs, from sub-micron to a few hundreds of microns in diameter and several centimeters in length. (ii) The refractive indices of thermotropic liquid crystals (8CB: $n_{o}=1.516, n_{e}$ $=1.673)$ are significantly higher than that of the surrounding medium containing water $(n=1.33)$ and CTAB $(n=1.435)$. (iii) Smectic filaments can be easily manipulated and produce desired structures by using laser tweezers ${ }^{1}$ (Fig. 74). (iv) Their smectic nature should substantially decrease the degree of light scattering losses and enhance the surface smoothness, as the molecular orientational fluctuations are depressed in smectic phases. (v) On a molecular level, the chiral smectic phase of LCs leads to the spontaneous formation of the filaments (if the surface energies are very low) with the intrinsic helical organization of the internal director structure, whereas achiral smectic phases produce filaments with perfect

\footnotetext{
${ }^{1}$ This is also a disadvantage as the unconfined filaments are not stable for long time. We can circumvent this problem by placing the filaments in square channels with the desired structures.
} 
elongated radial structures (see Section 3.2.2). Due to the helical organization of molecules, chiral smectic filaments can be used as photonic bandgap waveguides while achiral smectic phase filaments can work as total internal reflection optical waveguides. (vi) Recently it has been shown that a Gaussian beam is transformed into a Laguerre-Gaussian beam after passing through a microdroplet of a nematic LC with a topological point defect [190]. This shows that the topology of the matter strongly influences the topology of light that is passing through the matter. Smectic filaments always exhibit a topological line defect of the winding number 1 along their major axis. As a result of this internal director structure, it is to be expected that the optical modes not only perfectly adjust with this defect, but also have topological singularity of the electromagnetic field.

\subsubsection{Waveguiding in smectic-A filaments}

The detailed description of the optical setup that was used for investigating the waveguiding properties of the smectic-A filaments is provided in Section 2.2.4. The light guiding properties of any microfiber is studied by launching the external light into the fiber and analyzing the transmitted light at the other end of it. But, due to the method that we employed to produce smectic-A filaments (sandwich method $^{2}$ ), it is quite difficult to follow this general procedure for studying their waveguiding properties. Therefore, the light guiding properties of freely floating $8 \mathrm{CB}$ filaments were studied by positioning a tightly focused $\mathrm{Ar}^{+}$laser beam on a hemispherical end-cap of the dye doped $8 \mathrm{CB}$ filament. Consequently, the fluorescently generated light at the excitation spot propagated along the filament and, thereby, the quality of waveguiding was indirectly obtained by measuring the fluorescent light that was leaking out of the filament. While this method allows for checking the fluorescent decay along the filament, it is not adequate enough for quantitative measurements of the quality of waveguiding.

Hemispherical end-caps of the smectic filaments act as perfect spherical lenses. As a result, different fluorescent patterns were clearly visible as we moved the focus of the $\mathrm{Ar}^{+}$ beam along the hemispherical cap of the microfiber (Fig. 75). A significant amount of the fluorescent light in the fiber was internally reflected at apparently very steep angles with respect to the major axis of the filament (Fig. 75(a-d)), whereas most of the generated light was leaked into the surroundings at small angles (Fig. 75(e-f)). These fluorescent patterns in fact represent the "spiralling" of light along the inner surfaces of the LC-aqueous interfaces instead of the usual "ray patterns" observed in coaxial fibers.

The following few simple experimental observations provide further information about the light guiding in smectic-A filaments. If we place the continuous wave $A r^{+}$beam anywhere on a smectic filament (the beam direction is perpendicular to the sandwich cell) other than its hemispherical caps, it seems as if no light guiding is possible as shown in Fig. 76(a). From Fig. 76(b), it is clear that even within the hemispherical caps, light guiding can only be initiated from the region highlighted in Fig. 76(c). These observations suggest that light guiding is effectively possible from small regions on the filament's surface. However, this problem can be overcome very easily and it is effectively possible to send light from any point on the surface of the filament. Fig. 76(d-f) shows the experimental setup which makes

\footnotetext{
${ }^{2}$ Instead of the sandwich method, it is easy to envisage that the capillary method provides confined single filaments where it should be easy to couple light into the filament and collect the transmitted light at the other end of it. Our experiments with single filaments in square glass capillaries were rather unsuccessful. This was due to the light leaking from the filaments into the glass capillaries. This problem can be avoided if the experiments are carried out in PDMS channels. This is because of the relatively lower refractive index of the PDMS (1.4) in comparison to the ordinary refractive index of $8 \mathrm{CB}$ (1.516).
} 

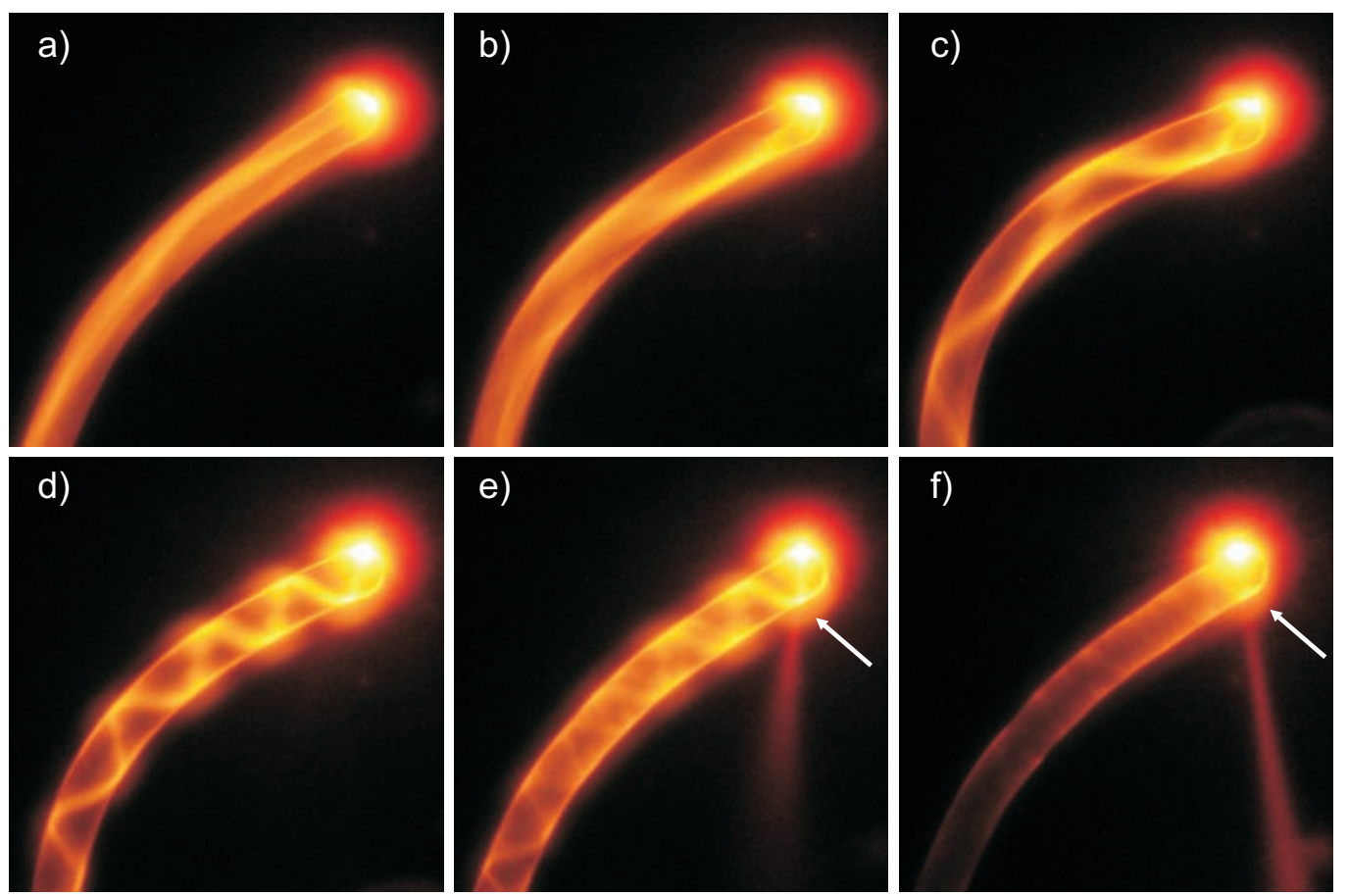

Figure 75: Light guiding through an $8 C B$ smectic-A filament doped with 0.01 wt\% Nile red. The surrounding aqueous solution contains $20 \mathrm{mM} \mathrm{CTAB}$. The power of the applied continuous wave $\mathrm{Ar}^{+}$laser beam for fluorescence excitation is $1 \mathrm{~mW}$. The spiral shaped trajectory of the guided light is changed according to the position of the laser beam on the hemispherical cap of the filament. (a-d) For these positions of the focused $\mathrm{Ar}^{+}$laser beam, very little of the fluorescent light is leaked into the surroundings as it is traveling along the $8 \mathrm{CB}$-aqueous interface. (e-f) A significant amount of light is leaking from the positions indicated by white arrows. Thus, the quality of light guiding is very bad for these positions of the laser beam on the hemispherical cap. These experiments suggest that the point of intersection of the major axis of the filament and its hemispherical cap is the best position for focusing the $\mathrm{Ar}^{+}$laser beam in order to achieve the best possible light guiding. Images are taken under crossed polarizers. Horizontal width of the images is $120 \mu \mathrm{m}$.

it possible to guide light from anywhere on the surface of the filament. This experiment clearly shows that there are no forbidden regions from where it is possible to guide light; instead it only depends on the incident angle of the $\mathrm{Ar}^{+}$beam.

One interesting problem is how light is guided at Y junctions. Our preliminary experiments show that by changing the position of the $\mathrm{Ar}^{+}$beam on the hemispherical cap of one arm, it is possible to switch light between either of the two arms selectively. As shown earlier, filaments can also wrap around each other very tightly and produce beautiful double stranded helical structures. Light guiding through these helical structures is apparently not so trivial, as shown in Fig. 77(a-c). It is possible to guide light selectively through one filament at a time as well as exclusively along the principal axis of the helical structure. It is not yet clear why the light has to guide along the helical axis of this self-assembled double stranded structure. Instead of forming helical structures, smectic filaments can just grow in a parallel manner as shown in Fig. 77(d). It is evidently clear from this figure that a significant amount of the fluorescent light from a filament (which is exposed to laser irradiation) leaks into its adjacent filament whenever the light in the exposed filament reaches the interface between two filaments. All of the above observations suggest that these self-assembled structures have enormous potential for photonic applications. 

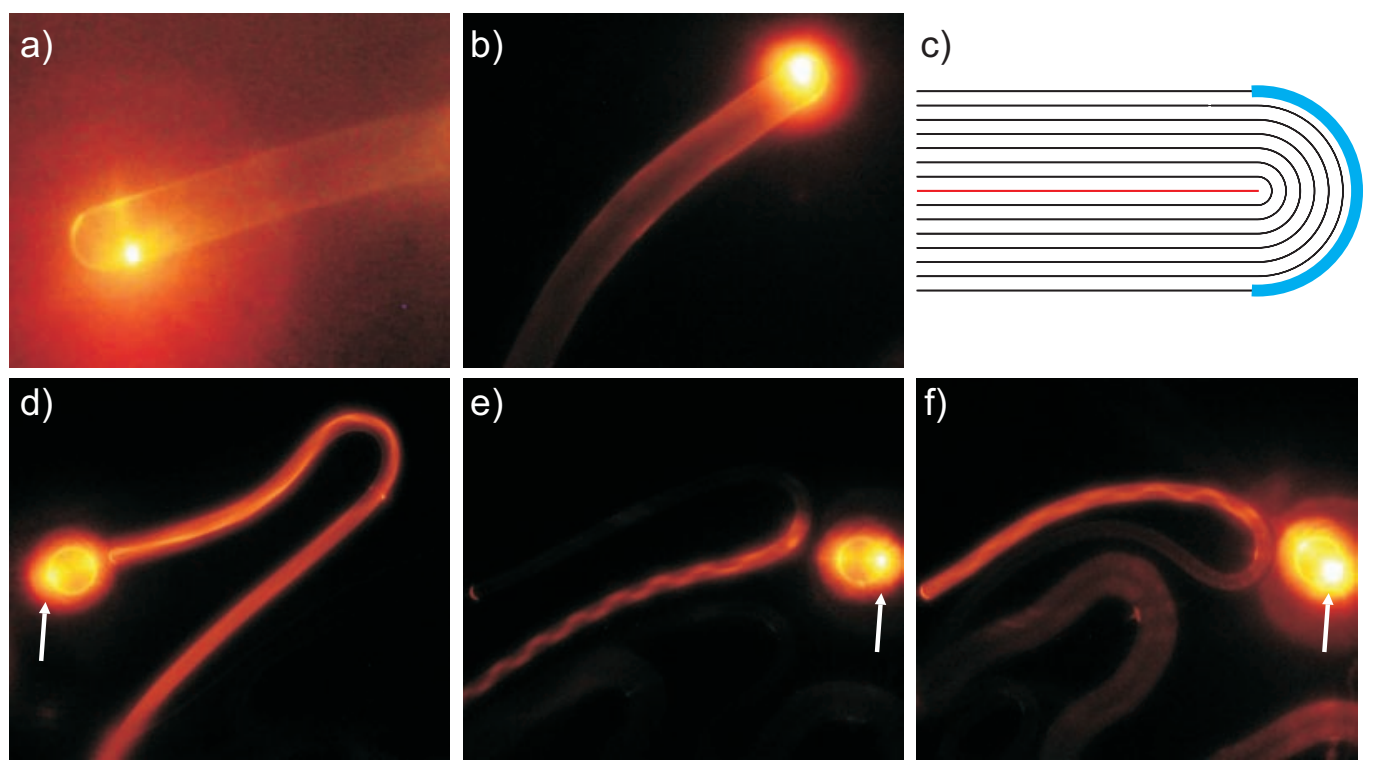

Figure 76: Possible regions on the surface of an $8 C B$ smectic- $A$ filament through which light can be guided. A continuous $\mathrm{Ar}^{+}$laser is used to excite the Nile red molecules in the filaments. The incident laser beam path is perpendicular to all images' planes including image 'c'. (a-b) Nile red doped $8 \mathrm{CB}$ filaments in $20 \mathrm{mM}$ CTAB aqueous solutions. These two images show that light cannot be guided even when the laser beam is focused onto a hemispherical cap of a filament, let alone anywhere else on the filament. Horizontal width of the images is $180 \mu \mathrm{m}$. (c) Figure 33 and the images 34 (a-b) apparently suggest that light guiding is possible only through the region marked with blue. This would seriously limit their practical applications. However, this is not entirely true. (d-f) Both droplets and filaments are doped with $0.01 \mathrm{wt} \%$ Nile red and the surrounding aqueous solution contains $5 \mathrm{wt} \%$ CTAB. Horizontal width of the images is $560 \mu \mathrm{m}$. An $A r^{+}$laser beam is focused at positions indicated by white arrows on $8 \mathrm{CB}$ smectic-A droplets. The leaking light from a droplet is no longer perpendicular to the image planes. It is evidently clear from the images that the leaking light from a droplet can be used to guide light from anywhere on the surface of a filament. It should be noted that images (e-f) represent light guiding in the same filament but from different positions on the surface of the filament.

Previously, thick filaments (cross-sectional diameter of a few tens to hundred $\mu \mathrm{m}$ ) were used to show spiraling patterns of the guided light. But to study the quality of light guiding, we have chosen thin filaments (cross-sectional diameter of a few $\mu \mathrm{m}$ ). They are chosen such that the complete hemispherical caps of the filaments can be easily illuminated uniformly with the incident laser beam. Fig. 78(a) shows a thin filament which is exposed to the continuous wave $\mathrm{Ar}^{+}$laser beam. It is clearly visible that the fluorescent light is emitted uniformly across and along the filament and a bright spot is observed at the other hemispherical end of the filament (Fig. 78(b)). Fig. 78(c-d) shows the measured intensity of the emitted fluorescent light along the filament. No substantial drop $(<5 \%)$ of the intensity is observed along the $\approx 360 \mu \mathrm{m}$ overall length of the filament if we exclude the regions surrounding both the hemispherical end-caps. A sudden spike in the fluorescence intensity value at the exit of the filament suggests that a significant amount of the guided light leaks into the surroundings at this position. Our preliminary experiments unsurprisingly show that the light leaking from the exit of the filament can further be used to guide light in a new adjacent filament.

LCs are birefringent and different polarizations of light sense different refractive indices. Thus, we could imagine that light guiding in the smectic filaments strongly depends on the 


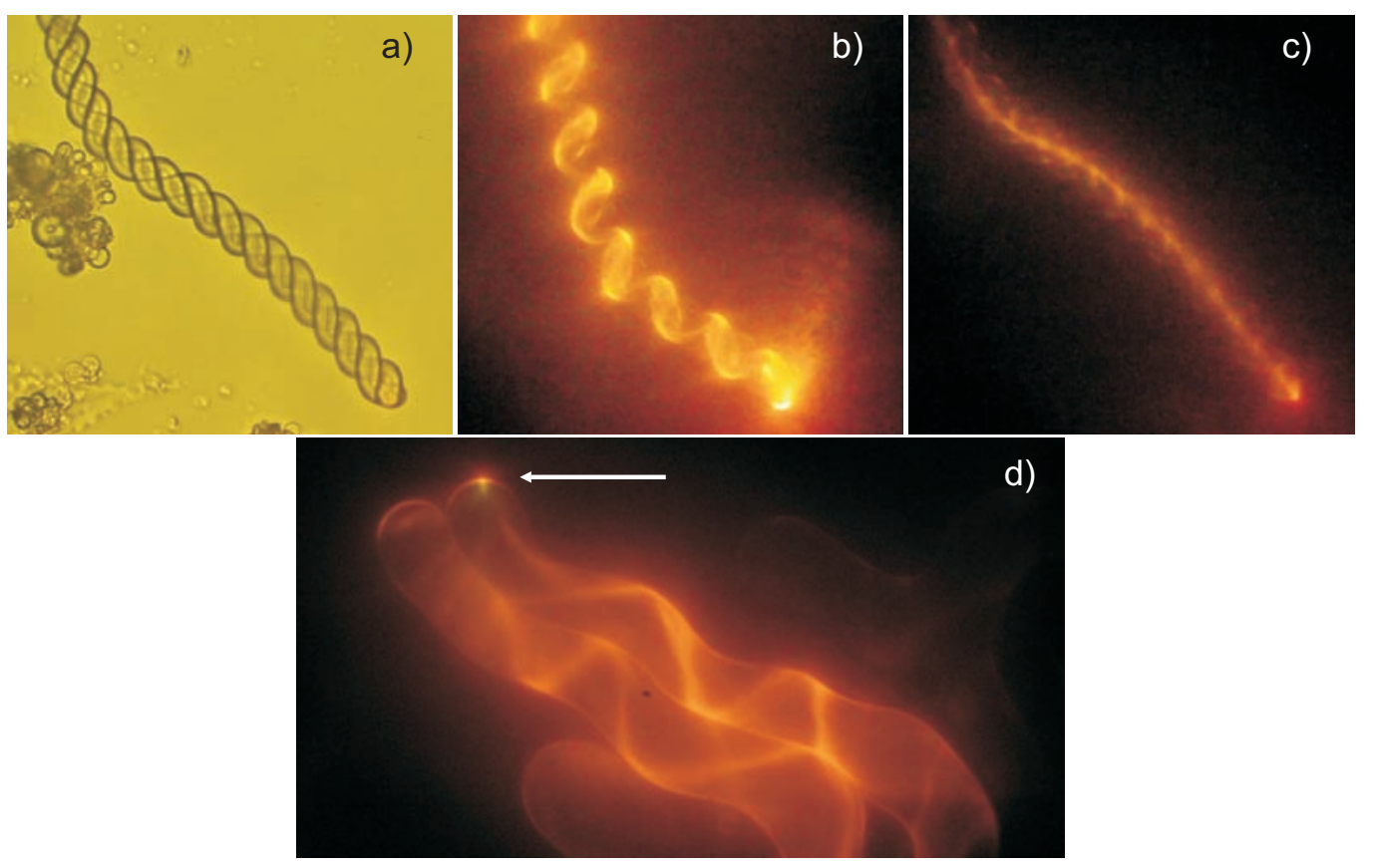

Figure 77: Light guiding in super structures formed by $8 C B$ smectic-A filaments. 8CB is doped with $0.01 \mathrm{wt} \%$ Nile red and the surrounding aqueous solution contains $80 \mathrm{mM} \mathrm{CTAB}$ and $0.5 \mathrm{M} \mathrm{NaCl}$. (a) White light image of a double stranded helix formed by two $8 \mathrm{CB}$ filaments. (b) A continuous $A r^{+}$laser beam is focused on one of the two loose ends of the double stranded helix. In this case, light is being guided in the exposed filament. (c) A continuous $\mathrm{Ar}^{+}$laser beam is focused exactly between the tips of two loose ends of the double stranded helix. Surprisingly, in this case, light is being guided along the principal axis of this structure. Horizontal width of the images (a-c) is $120 \mu \mathrm{m}$. (d) The white arrow shows the position of the $\mathrm{Ar}^{+}$laser beam on the hemispherical cap of a filament. Spiraling light patterns in these two adjacent filaments clearly indicate the light transfer at their interface. Horizontal width of the image is $250 \mu \mathrm{m}$.

polarization of the incident laser beam. Fig. 79 shows the measured intensity of the emitted fluorescent light along a smectic-A filament for different polarizations of the excitation beam $^{3}$. When the polarization of the excitation beam matches the major axis of the filament, we observe substantially enhanced fluorescence emission in comparison to any other combination. As shown earlier in Fig. 46, dye (Nile red) molecules are known to orient in the LC matrix so as to have the brightest fluorescence when the polarization of the excitation light is set parallel to the LC director. Thus, when the polarization of the incident beam matches the molecular orientation in the hemispherical cap as shown in Fig. 79(a), a maximum amount of the fluorescent light is generated in the exposed hemispherical endcap. This polarization dependence of the fluorescent light generation in the hemispherical end-caps also makes the intensity of the emitted fluorescent light along a smectic filament polarization dependent.

\footnotetext{
${ }^{3}$ The direction of the linearly polarized light is held constant and the filament is rotated in its plane accordingly in order to investigate the polarization dependence.
} 

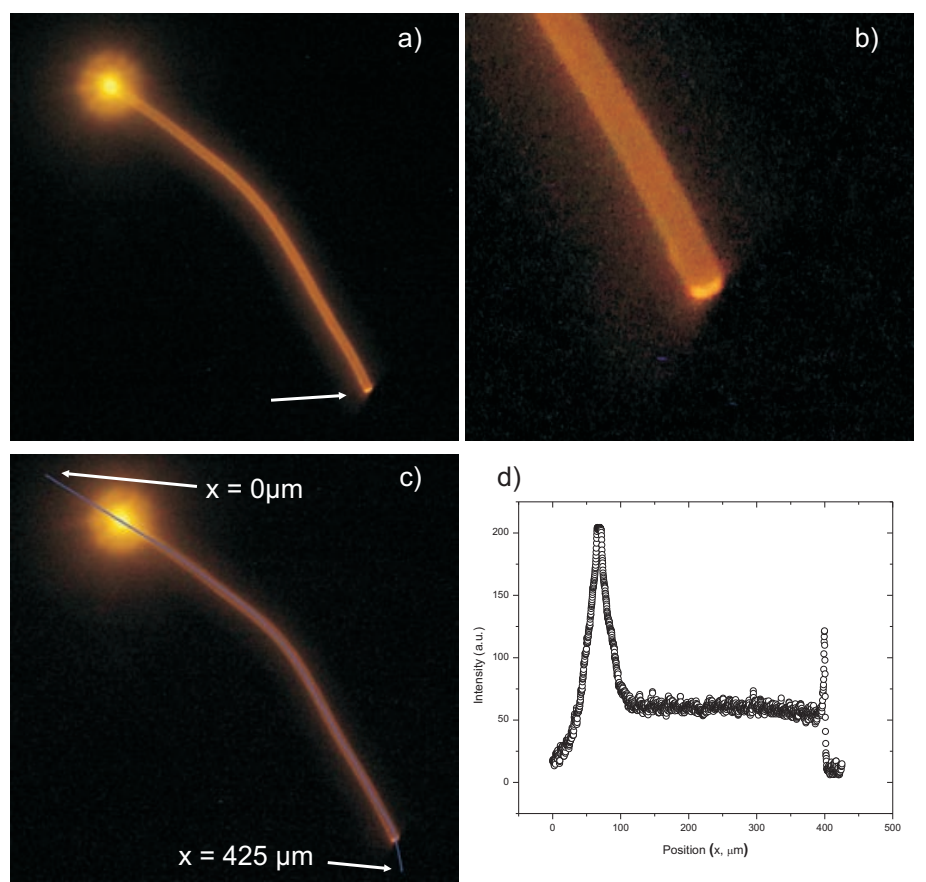

Figure 78: Measurement of the quality of light guiding in a thin $(8.5 \mu \mathrm{m}) 8 C B$ smectic- $A$ filament. $8 \mathrm{CB}$ filament is doped with $0.01 \mathrm{wt} \%$ Rhodamine-B and the surrounding aqueous solution contains $7.5 \mathrm{wt} \%$ CTAB. (a) The brightest spot in the image represents the position of the $\mathrm{Ar}^{+}$laser beam. The laser polarization is along the vertical direction in the plane of the image. The white arrow points to the leaking light at the exit of the filament. (b) Magnified view of the exit of the filament. (c-d) Intensity of light is measured along the path indicated by the blue line. Excluding the hemispherical caps of the filaments, the whole filament is glowing uniformly which indirectly suggests the excellent quality of light guiding.
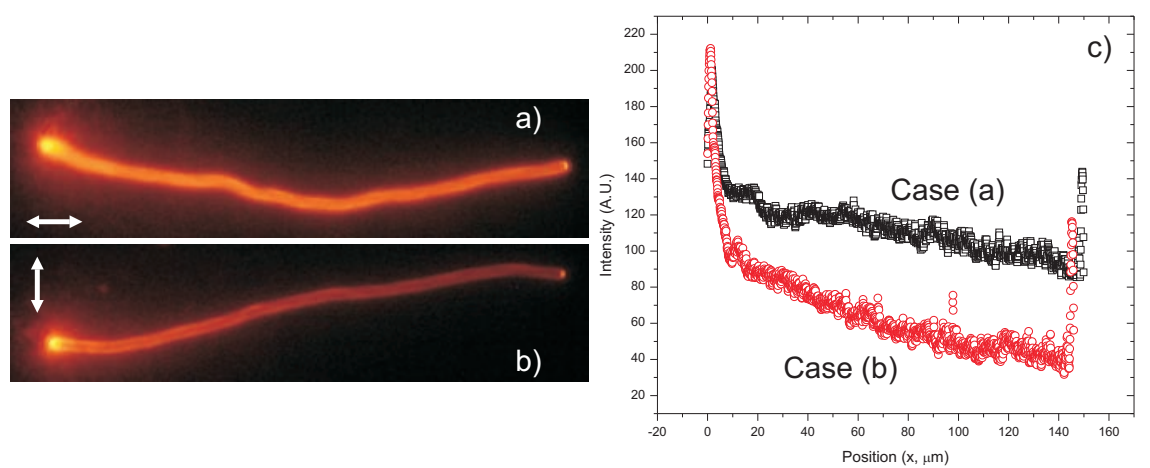

Figure 79: Measurements of the emitted fluorescence intensities along a thin $8 C B$ smectic- $A$ filament for different polarizations of the excitation $\mathrm{Ar}^{+}$laser beam. The concentration of $\mathrm{CTAB}$ in the surrounding aqueous solution is $20 \mathrm{mM}$. The cross-sectional diameter of the filament is $3.4 \mu \mathrm{m}$. The brightest spots and the double-sided white arrows in the images (ab) represent the positions of the excitation beam and its polarization directions. The slight difference in the filament's length is due to heating caused by prolonged exposure to the excitation beam (image (a) is taken after image (b)). (c) The excited region in each case is considered as a starting point $(\mathrm{x}=0 \mu \mathrm{m})$ for intensity measurements. The fluorescent light emitted from the exposed hemispherical caps causes its surroundings to glow apparently brighter than they should. This is the reason for the apparent low quality guiding observed in the filament. 


\subsubsection{Lasing in smectic-A filaments}

After studying the wave guiding properties of the dye-doped filaments, the next logical step was to investigate their lasing characteristics. The detailed description of the optical setup that was used for this purpose is provided in Section 2.2.5. The beam waist of the pulsed laser was adjusted to $\approx 20 \mu \mathrm{m}$. This laser beam was used to excite the dye-doped $8 \mathrm{CB}$ filaments at randomly chosen positions along the length of the filaments, as shown in Fig. 80. It is quite clear from the Fig. 80 (a) that at low pumping levels, the whole exposed volume of the filament was uniformly fluorescing without any signature of the lasing. As we gradually increased the pumping energy levels above a threshold, we observed the sudden onset of very intense and monochromatic reddish speckles shining from a very thin layer at the interface of $8 \mathrm{CB}$ filament and the aqueous solution, as shown in figure Fig. 80 (b). The appearance of the reddish speckles means that the $8 \mathrm{CB}$ filament started lasing. The pumping level at which lasing begins is known as lasing threshold. The intensity of these reddish speckles increased monotonously on increasing the levels of pumping energy. It is important to take note that the region that shines with reddish speckles extends far beyond the region of the filament that is exposed to the pulsed laser (Fig. 80(b,d)).

A close inspection of the lasing phenomenon in smectic-A filaments shows that the lasing originates from the resonant WGMs of light. The resonant WGMs circulate along the cross-sectional circumference of the filament, due to the total internal reflection (TIR) at the interface of $8 \mathrm{CB}$ filament and the aqueous solution. The resonant condition for a circulating mode is satisfied when it reaches the laser excitation position with the same phase after one circulation along the circumference of the cavity, such as a microsphere,
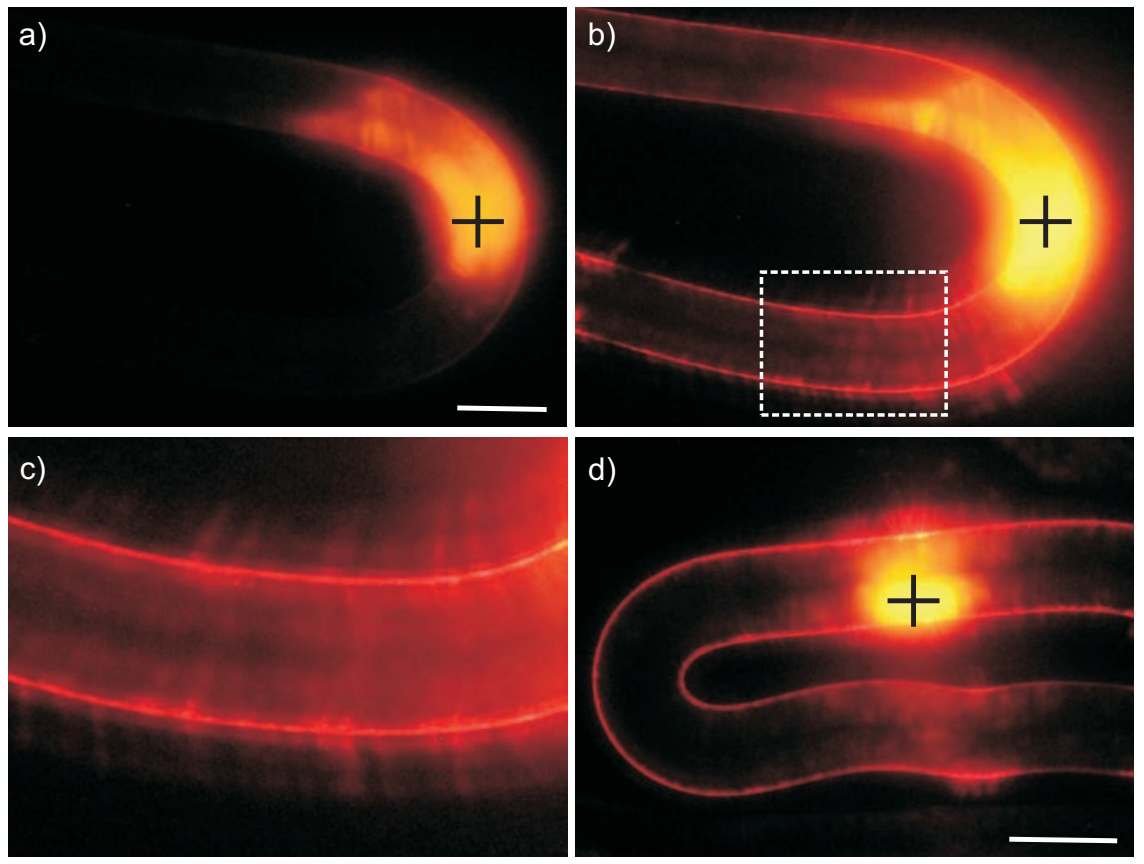

Figure 80: Lasing in Nile red dye doped $8 C B$ smectic-A filaments in a $100 \mathrm{mM}$ aqueous $C T A B$ solution. A pulsed laser beam is used to illuminate the $\approx 20 \mu \mathrm{m}$ diameter regions encircling the black crosses in images (a), (b) and (d). The laser polarization is along the vertical direction in the planes of the images. (a-b) The power of the pulsed laser is below the lasing threshold $\left(\approx 75 \mu \mathrm{J} / \mathrm{cm}^{2}\right)$ in case (a) and above the threshold in (b). Scale bar in image (a) represents $50 \mu \mathrm{m}$. (c) Magnified view of the region marked with a white color rectangle in image (b). (d) Another example of lasing in $8 \mathrm{CB}$ filaments. The observed lasing threshold for this configuration is $\approx 1.5 \mathrm{~mJ} / \mathrm{cm}^{2}$. Scale bar: $100 \mu \mathrm{m}$. 
microfiber, microdisc etc [191]. Because of TIR, the WGM cavities exhibit large Q-factors ${ }^{4}$, thereby enabling low-threshold lasing with very small mode volume [176,191-193]. If we look at the lasing cavity under a microscope, we observe the lasing speckles from the thin interfacial region of the cavity [104]. The smectic-A filaments, having characteristic features of high refractive index and a circular cross-section, represent cylindrical microresonators. This means WGMs circulate in a plane perpendicular to the major axis of the filament. As a result, the leaked light from the circulating modes leaves the cross-sectional interface tangential to it's surface, in both directions. Therefore, we see reddish monochromatic speckles emanating only from the two edges of the filament (Fig. 80(b-d)). Because of the cylindrical symmetry of the filaments, we obtain identical pictures from all angles of observation.

The spectrum of lasing speckles was analyzed at various pumping energy levels and is shown in Fig. 81(a-b). At low pump powers of the pulsed laser (where no speckles appear along the LC-aqueous interface), no WGMs are observed in the spectrum. Nor are they (WGMs) observed, when a filament is excited with a continuous wave $A r^{+}$laser. As the speckles start to appear at higher pump powers, we observed a set of sharp spectral lines superposed on a broad fluorescent background, indicating the onset of lasing. These lines can clearly be attributed to the WGMs circulating along the cross-sectional circumference on the inner side of the smectic-A filament - aqueous interface. For pump powers higher than the lasing threshold, some modes in the wavelength region where the dye has the highest gain, start to lase. This lasing behavior is very similar to the manner in which a bipolar nematic droplet lases but an important difference still exists [194]. A bipolar nematic droplet also does not show WGMs at low pump powers due to low Q-factors ${ }^{5}$. But when it starts to lase, instead of single lines, the modes in the bipolar nematic droplet are split and are present as groups of spectral lines in the spectrum. The mode splitting in bipolar nematic droplets can be explained due to the lack of symmetry in the optical path. No mode splitting is observed in perfect cylindrical filaments due to the spherical symmetry in the optical path.

As we observed the strong relation between the light polarization and the wave guiding in smectic-A filaments, we also investigated lasing characteristics for different orientations of a linearly polarized pumping beam with respect to the long axis of the filaments ${ }^{6}$. When the polarization of the pumping beam is parallel to the major axis of the filament, the lasing threshold is $\approx 75 \mu \mathrm{J} / \mathrm{cm}^{2}$ (Fig. $81(\mathrm{c})$ ). This value is comparable to the lasing threshold for WGM lasing in spherical radial nematic droplets $\left(0.25 \mathrm{~mJ} / \mathrm{cm}^{2}\right.$ for a $13.7 \mu \mathrm{m} 5 \mathrm{CB}$ droplet) [182] and is also comparable to recently reported low-threshold lasing in dye-doped polymer fibers ( $53 \mu \mathrm{J} / \mathrm{cm}^{2}$ for a fiber with $32 \mu \mathrm{m}$ diameter) [195]. But surprisingly, this value is much lower than the threshold values observed for radial $8 \mathrm{CB}$ smectic-A droplets $(1.29$ $\mathrm{mJ} / \mathrm{cm}^{2}$ for a $41.2 \mu \mathrm{m} 8 \mathrm{CB}$ droplet). When the polarization of the pumping beam makes an angle of $45^{\circ}$ or $90^{\circ}$ to the filament axis, the observed thresholds are at $\approx 0.9 \mathrm{~mJ} / \mathrm{cm}^{2}$ and $\approx 2 \mathrm{~mJ} / \mathrm{cm}^{2}$ respectively (Fig. 81(d-e)). As shown in Fig. 81(f), the lasing threshold apparently does not depend upon the cross-sectional diameter of the filaments. However, as the lasing thresholds of smectic filaments are very small, the current setup does not allow us to obtain the exact values of lasing thresholds (the minimum power produced by our pulsed

\footnotetext{
${ }^{4}$ The Q-factor values represent the amount of time for which the light is in the resonator (i.e., the plane perpendicular to the major axis of the filament in which fluorescent light is trapped).

${ }^{5}$ In case of radial nematic or smectic-A droplets, WGMs are observed even below their respective lasing thresholds.

${ }^{6}$ We changed the direction of the linearly polarized pulsed beam at the sample holder by rotating the linear polarizer which lies right after the quarter wave plate in the experimental setup (see Section 2.2.5 for complete details of the experimental setup)
} 

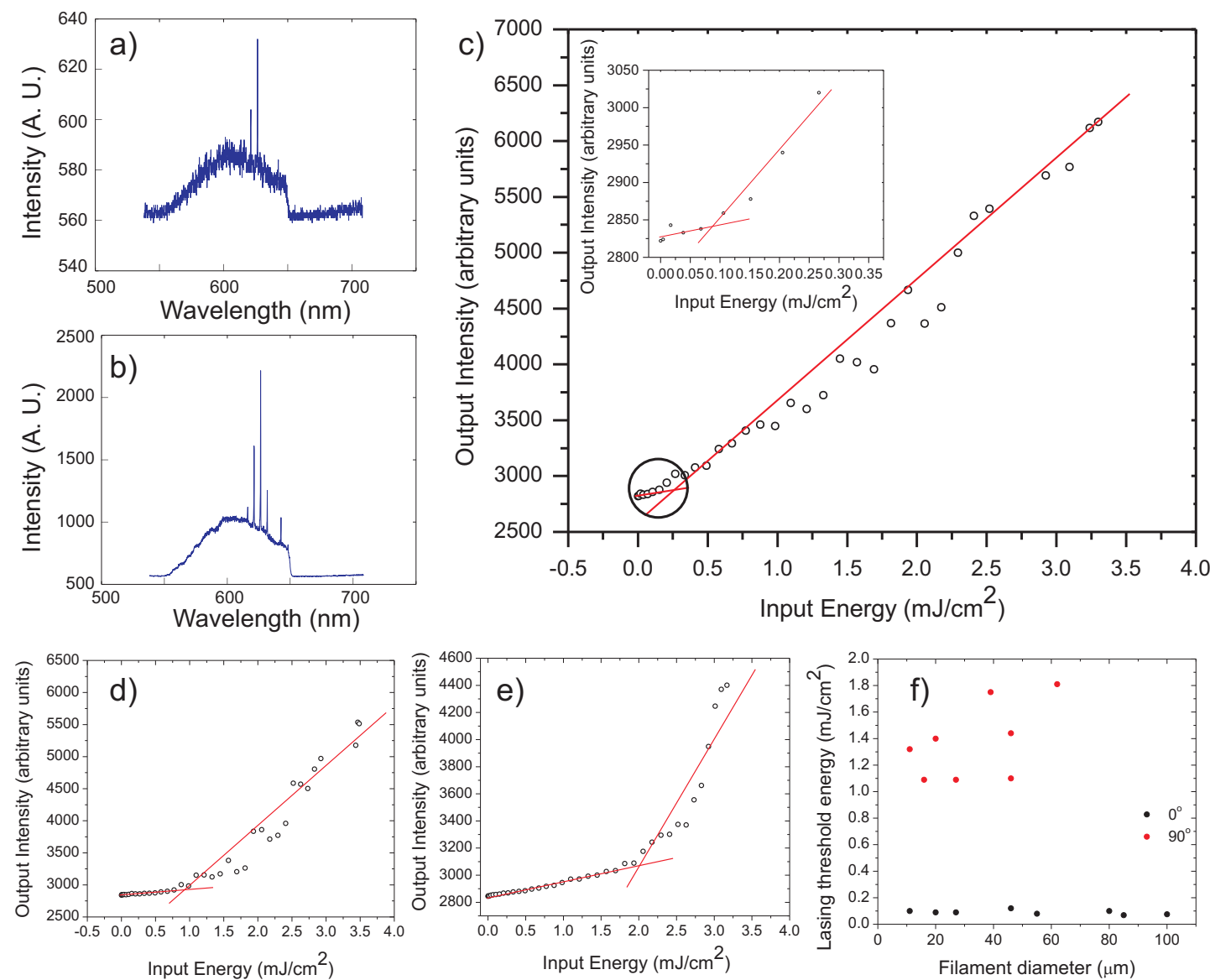

Figure 81: Effect of the pulsed laser polarization on lasing threshold values of 8CB smectic$A$ filaments. $8 \mathrm{CB}$ filaments are doped with $0.01 \mathrm{wt} \%$ Nile red and the aqueous solution contains $100 \mathrm{mM} \mathrm{CTAB}$. (a-c) The polarization direction of the linearly polarized pumping pulsed laser is along the major axis of the filament. Images (a) and (b) represent lasing spectra for input energies just above and well above the threshold for lasing respectively. The cut-off at $650 \mathrm{~nm}$ in the lasing spectra is due to the IR cut-off filter inserted in the dual beam laser tweezers. Image (c) represents the plot of the emitted spectral line's intensity versus the laser excitation energy. The inset in the image (c) presents the enlarged view of the encircled region around the lasing threshold. The observed lasing threshold is the minimum for this combination of laser polarization and the filament's major axis directions. (d) The experiment is repeated again after changing the angle between the polarization of the pulsed laser and the filament's major axis to $45^{\circ}$. (e) In this case, the angle between the polarization of the pulsed laser and the filament's major axis is $90^{\circ}$. The laser line width is not polarization dependent as we observed the same $\approx 0.2 \mathrm{~nm}$ width in all of the above three cases. All the red lines are just a guide for the eye. (f) The lasing threshold values observed for various filaments are plotted against their respective cross-sectional diameters.

laser is $\left.\approx 10 \mu \mathrm{J} / \mathrm{cm}^{2}\right)$. An ultra-low power pulsed laser is needed to confirm this observation. Ultra-low pumping powers can also be achieved by inserting a neutral density filter in the current optical setup. Unfortunately the polarization state of the reddish speckles is not yet investigated in detail. In any case, no significant change in the intensity of reddish speckles was observed as the polarizer was rotated. Interestingly, the laser light emitted from the surface of a lasing filament can also cause other filaments or droplets to lase when they are brought into contact with the lasing filament (Fig. 82). 

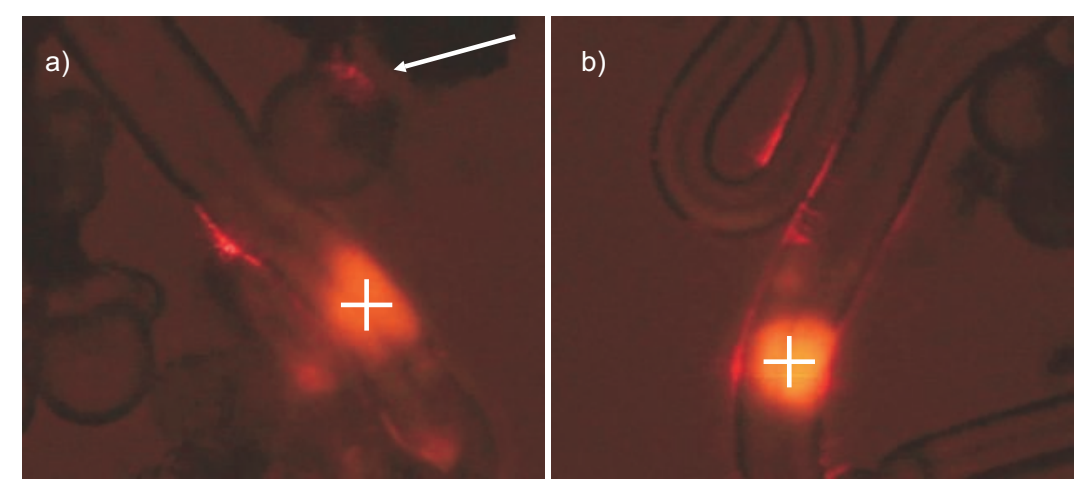

Figure 82: Coupled WGM cavities (8CB filaments and droplets). 8CB smectic-A filaments and droplets are produced in a CTAB 5 wt $\%$ aqueous solution at $23^{\circ} \mathrm{C} .8 \mathrm{CB}$ is doped with $0.01 \mathrm{wt} \%$ Nile red. A pulsed laser beam is used to illuminate the regions marked with white crosses in both images. The polarization of the laser beam is along the vertical direction in the planes of the images. (a) Fluorescent laser light from a filament is coupled into a droplet when it physically touches the filament. As expected, a bright lasing spot (reddish speckles) is observed at the diametrically opposite end of the contact between the droplet and the filament as indicated by a white arrow. This bright spot on the smectic-A droplet confirms the light coupling between these two objects. (b) Coupling is also observed between two adjacent filaments with different diameters. Horizontal width of the images is $400 \mu \mathrm{m}$. Images are taken under slightly uncrossed polarizers.

\subsubsection{Optical properties of smectic- $C^{*}$ objects (droplets and filaments)}

The confinement of light is realized in all achiral LC objects such as smectic-A droplets and filaments through the total internal reflection phenomenon. Light can also be confined much more efficiently by using photonic structures. A periodic modulation of the refractive index exists in the photonic structures, which results in a selective reflection band. The frequency range of this reflection band depends on the refractive indices of the material and the period of the photonic structure. This reflection band is also known as the photonic bandgap (PBG). The frequencies in a PBG are forbidden inside its respective photonic structure. Cholesteric liquid crystals (CLCs) and smectic-C* liquid crystals are formed by rod-like molecules whose directors are self-organized in helical structures (Fig. 6). In the planes perpendicular to the helical axis, the LC directors are continuously rotated along the helical axis. These self-organized helical structures act as PBG materials provided that their helical pitch $\mathrm{p}$ is in the same order of magnitude as the wavelength of the light.

Quite a few studies have been conducted to uncover the optical properties of CLCs. For example, photonic band-edge dye lasing is observed in planar cholesteric cells and cholesteric droplets $[105,196]$. Cholesteric droplets are particularly interesting and their structure is also known as a spherical Bragg-onion resonator. They have omnidirectional PBG due to spherical symmetry. The observed dye lasing in cholesteric droplet is also omnidirectional due to its omnidirectional PBG. This omnidirectional laser emits a coherent laser light uniformly into full space which means it acts as a 3D laser. It would also be interesting to investigate the optical properties of our laboratory's recently discovered cholesteric filaments (not discussed in this thesis).

The optical properties of the smectic- $\mathrm{C}^{*}$ objects such as droplets and filaments are not yet reported. In the following sections, the optical properties of smectic- $\mathrm{C}^{*}$ objects are described. The helical pitch values of all the investigated smectic- $\mathrm{C}^{*}$ objects are maintained in such a way that a significant amount of the fluorescent light emitted by Nile red could be trapped inside these objects. 


\section{Smectic-C ${ }^{*}$ droplets:}

Dye-doped smectic- $\mathrm{C}^{*}$ droplets also show WGMs when they are excited by a continuous wave $\mathrm{Ar}^{+}$laser. However, there is a big difference in the way light propagates inside these PBG materials. Inside the LC droplets formed by achiral rod-like molecules, a resonant condition is achieved by light circulation along the circumference of the droplet. Light does not get trapped anywhere except near the surfaces of these LC droplets. However, confinement of light can be achieved even inside the droplet in the case of smectic-C* droplets, as shown in Fig. 83. The light circulation path shrinks as we move the laser beam position from the edge to the center of the smectic- $\mathrm{C}^{*}$ droplet $^{7}$. A bright spot of fluorescent light is observed at the point of the laser beam as well as at the opposite end of the light circulation path.

In the case of a radial nematic or smectic-A droplet, the confined light circulates only in the plane that contains both the incident laser beam and the point defect of the droplet [194]. Whereas, in the case of a smectic- $\mathrm{C}^{*}$ droplet, the confined light additionally circulates in the plane that is perpendicular to the incident laser beam, and which also contains the point defect of the droplet.

It is known that the free spectral range $(\mathrm{FSR})^{8}$ of a radial nematic or smectic-A droplet increases with the decreasing diameter of the droplet. If we make the analogy with smectic$\mathrm{C}^{*}$ droplets, one would predict that the FSR should increase with a shrinking light circulation path (i.e., as we move the position of the laser beam from the edge to the center of the droplet). On the contrary, FSR value remains constant irrespective of the radius of the light circulation path ${ }^{9}$. To sum it up, many unanswered questions still persist regarding the confinement of light in a smectic- $\mathrm{C}^{*}$ droplet.
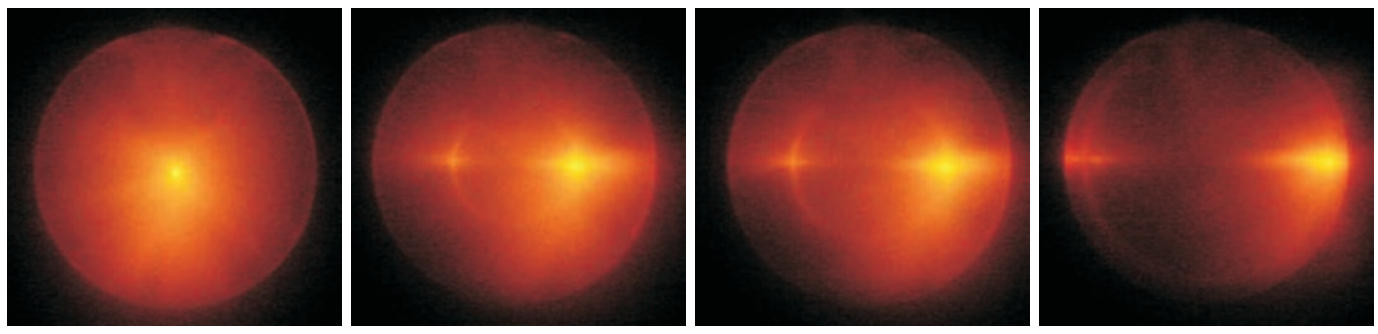

Figure 83: Circulation of fluorescent light in a smectic- $C^{*}$ droplet. This $100 \mu \mathrm{m}$ smectic-C* droplet is prepared with a LC mixture which contains $74 \mathrm{wt} \% \mathrm{C} 7,25 \mathrm{wt} \% 8.0 .6$ and $1 \mathrm{wt} \%$ monoolein. This LC mixture is doped with $0.01 \mathrm{wt} \%$ Nile red. The surrounding aqueous solution contains $1.5 \mathrm{mM}$ CTAB. A continuous wave $A r^{+}$laser beam is used to excite the dye molecules. The laser beam's optical path is perpendicular to the planes of the images. The polarization of the laser beam is along the vertical direction in the planes of the images. The brightest spots in the images represent the positions of the laser beam. We observed the same FSR values for these circulation paths (with different diameters) of the emitted fluorescent light.

\footnotetext{
${ }^{7}$ Surprisingly, cholesteric droplets do not display this behavior.

${ }^{8}$ FSR means the spacing between two neighboring Whispering Gallery modes, which depends on the length of the optical path.

${ }^{9}$ But one should note that FSR increases with a decreasing smectic-C* ${ }^{*}$ droplet diameter just as in the case of radial nematic droplets.
} 


\section{Smectic- ${ }^{*}$ filaments:}

The three-dimensional structure of the smectic- $\mathrm{C}^{*}$ filaments is shown in Fig. 51. Filaments are nothing but elongated droplet structures. Thus, one can assume that the confinement of light in filaments will not be much different from the circulation of light in droplets. Light guiding in smectic- $\mathrm{C}^{*}$ filaments is investigated using the same experimental method that was employed for smectic-A filaments. Light guiding properties are investigated by exposing a dye-doped freely floating $\mathrm{C} 7$ filament to a continuous wave $\mathrm{Ar}^{+}$laser and observing the propagation of fluorescent light along the filament. Here as well, the fluorescent light splits into two parts as it was in the case of smectic-C* droplets (Fig. 84(a-c)). One part of the light circulates in a plane that is perpendicular to the incident laser beam, and which also contains the line defect. This in-plane light circulation path is an elongated version of the light circulation path observed in a smectic- $\mathrm{C}^{*}$ droplet. It also shrinks as the incident light beam is moved from the edge to the center of the hemispherical cap. In this experimental configuration, light guiding is only possible if the position of the laser beam lies anywhere on the surfaces of the hemispherical caps of a filament.

In order to visualize the second type of light propagation, let us remove the smectic- $\mathrm{C}^{*}$ layers which lie outside the first path of the fluorescent light. The remaining part of the smectic-C* filament apparently behaves just like a smectic-A filament. This is due to the fact that we observe the same spiral shape fluorescent light patterns here as well, as it was in the case of smectic-A filaments. As we move the position of the laser beam along a hemispherical cap of this hypothetical filament, fluorescence patterns also change just as in the case of smectic-A filaments. It is reasonable to assume that both types of circulation of light are a result of a selective reflection phenomenon because smectic- $\mathrm{C}^{*}$ filaments are PBG structures. More detailed investigation is necessary for a complete understanding of light guiding in smectic- $\mathrm{C}^{*}$ filaments.

Since the helical pitch of the smectic-C* materials can be easily modified with external thermal and electrical fields, thereby, PBG tuning in smectic- $\mathrm{C}^{*}$ filaments is possible. Preliminary experiments are conducted to investigate the thermal PBG tuning in smectic- $\mathrm{C}^{*}$ filaments. As shown in Fig. 84(d), a smectic- $\mathrm{C}^{*}$ filament is produced in a $50 \mu \mathrm{m}$ square capillary. The filament is attached to its bulk reservoir which contains numerous defects. It is important to take note that the filament does not contain any dye. The hemispherical cap of the filament is exposed to a white laser source which contains a broad emission spectrum of light from $470 \mathrm{~nm}$ to $830 \mathrm{~nm}$. Depending on the pitch, only part of the light is coupled into the filament and travels along the filament until it reaches the bulk reservoir. Due to the presence of defects, transmitted light is severely scattered as it passes through the bulk reservoir. Scattered light from the bulk reservoir is collected and analyzed using a high-resolution imaging spectrometer. The helical pitch of the smectic- $\mathrm{C}^{*}$ materials increases with increasing temperature and becomes infinity at Smectic- $\mathrm{C}^{*}$ - Smectic-A transition temperature. This means, an increase in temperature should lead to red shift of the PBG. Our preliminary experiments show the same expected thermal behavior which suggests that smectic- $\mathrm{C}^{*}$ filaments could find a potential application as tunable photonic band-gap waveguides. 

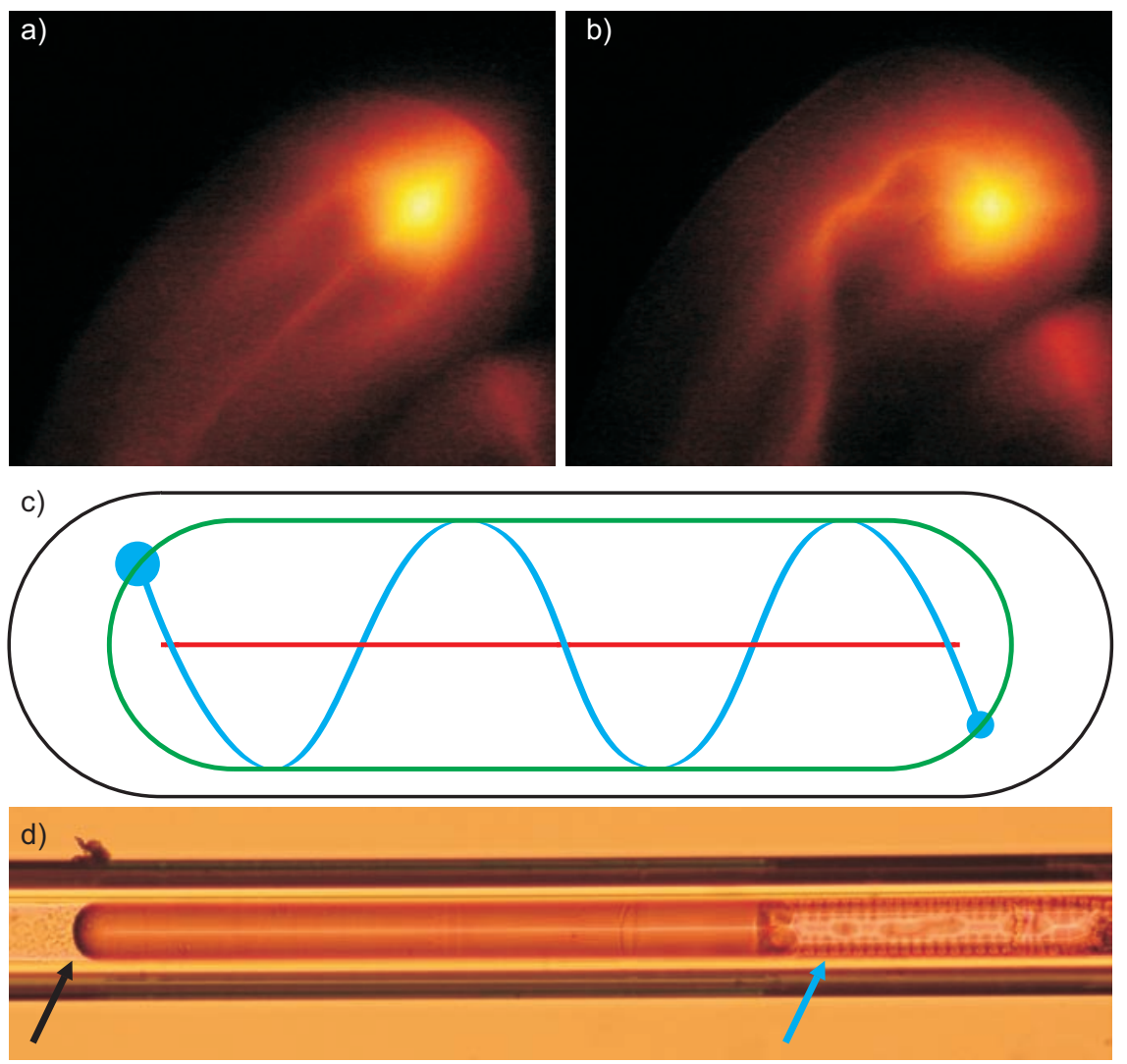

Figure 84: Light guiding through $C^{17}$ smectic- $C^{*}$ filaments. (a-b) The same LC mixture from Fig. 83 is used to produce smectic- $\mathrm{C}^{*}$ filaments. The same experimental setup described previously in Fig. 83 is used again to investigate the light guiding in smectic- $\mathrm{C}^{*}$ filaments. The brightest spots in the images represent the positions of the $\mathrm{Ar}^{+}$laser beam. Both types of fluorescent light propagation are clearly visible in both images. (c) Schematic representation of light guiding in a smectic- $\mathrm{C}^{*}$ filament based on experimental observations. The black color structure with an orange line as its major axis represents the top view of a smectic-C $\mathrm{C}^{*}$ filament. Green and blue color structures represent the first and the second types of the fluorescent light propagation in the filament respectively. The bigger blue dot represents the position of the $\mathrm{Ar}^{+}$laser beam. The leaking light at the other end of the filament is depicted with the smaller blue dot. (d) This C7 filament (doped with 1 wt\% monoolein) is produced in a $1.5 \mathrm{mM}$ aqueous CTAB solution. The black arrow indicates the position of the incident white-light laser source. The blue arrow indicates the bulk $\mathrm{C} 7$ reservoir from where the transmitted light is collected. 


\section{Conclusions}

In this dissertation, a study of the interactions between thermotropic LCs and aqueous ionic surfactant solutions was presented. Unlike the interactions between thermotropic LCs and solid substrates (display devices), the interactions between thermotropic LCs and other immiscible liquids (water, glycerol, silicone oils and fluorinated oils, etc.) are not that well understood. Especially, investigating the thermotropic LC-aqueous interfaces might be exceptionally rewarding. This is due to the fact that an understanding of the thermotropic LC-aqueous systems may lead to a greater understanding of the complex lyotropic liquid crystal systems, which are the basis for life itself. For example, consider the case of lyotropic myelin figures. Lyotropic liquid crystals (through myelin figures) were the first liquid crystals to be discovered in the 1850s. Lyotropic myelin figures have been observed in various systems until now but not much progress has been made in the understanding of their origins. Lyotropic myelin figures, i.e., multi-lamellar cylindrical filaments, are formed when an amphiphilic lamellar phase comes into contact with excess solvent. They form at the interface between the lamellar phase and the excess solvent and they grow into the excess solvent side of the interface. We observed that some unreported structures may also grow into the other side of the interface (not reported in this thesis). The presence of the solvent on both sides of the interface makes it difficult to understand the system. We have shown in this thesis that working with an analogous system of thermotropic smectic LCs in aqueous surfactant solutions greatly simplifies the problem and enhances our understanding of the formation, growth and stability of these filament-like structures.

In the first part of the thesis, the interactions between the most simple thermotropic liquid crystals - nematic LCs - and aqueous ionic surfactant solutions were investigated. The concentration of an ionic surfactant was always chosen such that its combination with water does not form any lyotropic LCs. For example, all the chosen concentrations of TTAB were well below $40 \mathrm{wt} \%$. This is due to the fact that TTAB forms lyotropic liquid crystals with water at concentrations above $40 \mathrm{wt} \%$. First, the equilibrium phase diagrams of various nematic LC-aqueous TTAB systems were prepared, which are essential to know the minimum energy states of the respective systems. The next logical step is to investigate the routes through which non-equilibrium nematic LC-aqueous systems attain their respective equilibrium states. This study was carried out by introducing a few hundred $\mu \mathrm{m}$ sized droplets (i.e. parent droplets) into aqueous ionic micellar solutions and tracking them with a video recorder. The micellar solubilization behavior of the nematic droplets is very similar to the isotropic droplets. In both cases, we observe a linear decrease of the parent droplet size despite the material transfer across the droplet's surface in both directions. This means that the material transferred from the parent droplet outweighs the aqueous solution that is transferred into the droplet during the entire solubilization process. The material transfer from the parent droplet ceases once the active micelles in the surrounding aqueous solution are exhausted. The aqueous solution that is transferred into the parent droplet appears as a few micron-sized droplets (i.e. aqueous daughter droplets). In the case of nematic 
parent droplets, the aqueous daughter droplets have the tendency to form chains due to the mutual interactions mediated by the elastic deformation of the nematic director field they induce. The behavior of the nematic droplets in aqueous solutions deviates significantly from the isotropic droplets when the interfacial tension values are greatly reduced. Our experiments with various nematic LCs show that for a given nematic LC-aqueous system, there might exist a temperature below which the nematic parent droplet either expels tiny nematic daughter droplets or transforms itself into a filament-like structure. This type of behavior is totally absent in the case of isotropic parent droplets.

During the entire micellar solubilization process, neither a nematic nor an isotropic parent droplet remains stationary. The material transfer across a parent droplet's surface induces convective flows both inside and around the parent droplet. The hydrodynamic flow field around the droplet can propel the parent droplet itself. The speed of the selfpropelled parent droplet increases with increasing surfactant concentration. Both nematic and isotropic self-propelled droplets exhibit translational motion only. In contrast, the cholesteric droplets display an extra rotational motion in addition to the translational motion. As it is extremely easy to produce these artificial swimmers in monodisperse form as well as in huge quantities, we believe that this type of microswimmer is a promising experimental model system for the study of squirming swimmers and their collective behavior.

In the second part of the thesis, we investigated the behavior of the fluid thermotropic smectic LCs in aqueous ionic micellar solutions. As in the case of nematic LCs, the smectic LCs also completely solubilize in aqueous ionic micellar solutions, provided there are enough micelles to accommodate all the LC molecules. During the solubilization of smectic droplets, smectic filaments may form spontaneously at the smectic droplet-aqueous interfaces. The smectic filaments form due to the competition between the elastic free energy and the interfacial energy. The presence of defects in the smectic droplets and the ultra-low interfacial tension values are the necessary and sufficient conditions for the formation of the smectic filaments. The structural analysis of the smectic-A filaments reveals that the LC molecules are oriented perpendicular to the surface of the filament and the layers are wrapped around its major axis. Other fluid smectic phases produce similar cylindrical structures with the only difference being in the molecular arrangements. For example, a helical arrangement of the LC molecules is observed in the case of smectic- $\mathrm{C}^{*}$ filaments. It is important to note that the filaments are devoid of any material from their surrounding aqueous solutions. The smectic filaments are extremely flexible due to the small ratios of bending to compression moduli, i.e., the energy related to a bending deformation of the layers is several orders of magnitude smaller than that related to a compression of the layers. The smectic filaments are only transient structures, they either retract back into the parent smectic droplet or spontaneously eject tinier filaments (LC ropes) into the surrounding aqueous solution. The LC ropes can also be artificially produced by creating defects in the surfaces of the filaments, using a microfiber or a short heat pulse generated by optical tweezers. The LC ropes may further transform either into vesicles or into a beautiful beads-on-a-string structure (Section 3.2.4.4), depending on the salt concentration in the surrounding aqueous solution. In highly concentrated surfactant solutions, the smectic droplets often eject tiny smectic daughter droplets instead of producing filament-like structures at their surfaces. We observed that these ejecting smectic droplets self-propel themselves using the thrust provided by this droplet ejection process. In order to realize any practical applications of the smectic filaments, it is imperative to find ways to stabilize them. We have shown that it is possible to stabilize these transient structures for at least several months, either by confining them in square channels or by using a UV photo polymerization technique.

Finally, we demonstrated the optical applications of the smectic filaments. We success- 
fully showed the light guiding through the smectic-A filaments as well as wishpering gallery mode lasing in a plane perpendicular to the smectic-A filaments. Due to the birefringent nature of the LCs, both light guiding and lasing phenomena depend significantly on the polarization of the excitation laser beam. The best quality of light guiding as well as ultralow lasing thresholds were achieved when the polarization of the excitation laser beam was parallel to the major axis of the smectic-A filament. The light guiding properties of several types of structures formed by smectic-A filaments (Y junctions, double stranded helical structures) were also briefly discussed. Due to the helical arrangement of the LC molecules in the smectic-C* filaments, we found that they behave like photonic band-gap waveguides. As the smectic filaments have line defects along their major axes, they are likely to support Gaussian-Laguerre eigenwaves.

Previously, many optical applications of self-assembled spherical cavities were reported. But, we need many more different self-assembled structures in order to realize soft photonic circuits. Such a soft photonic circuit has several advantages over hard matter photonic circuits such as tunability, self-healing and real time reconfigurability. We believe our work on smectic filaments took us a step closer to realizing this dream. It would be highly desirable to look into the optical properties of nematic, cholesteric and blue phase filaments. 


\section{References}

[1] Addy Pross and Robert Pascal. The origin of life: what we know, what we can know and what we will never know. Open biology, 3(3), 2013.

[2] C. M Bradford, A. D Bolatto, P. R Maloney, J. E Aguirre, J. J Bock, J Glenn, J Kamenetzky, R Lupu, H Matsuhara, E. J Murphy, et al. The water vapor spectrum of APM $08279+5255$ : X-ray heating and infrared pumping over hundreds of parsecs. The Astrophysical Journal Letters, 741(2):L37, 2011.

[3] Berthold-Georg Englert. Lectures on quantum mechanics, volume 3. World Scientific, 2006.

[4] Sun Kwok and Yong Zhang. Mixed aromatic-aliphatic organic nanoparticles as carriers of unidentified infrared emission features. Nature, 479(7371):80-83, 2011.

[5] Sylvie Derenne and François Robert. Model of molecular structure of the insoluble organic matter isolated from Murchison meteorite. Meteoritics \& Planetary Science, 45(9):1461-1475, 2010.

[6] S. P Balm, J. P Hare, and H. W Kroto. The analysis of comet mass spectrometric data. Space Science Reviews, 56(1-2):185-189, 1991.

[7] Stanley L Miller et al. A production of amino acids under possible primitive earth conditions. Science, 117(3046):528-529, 1953.

[8] Stanley L Miller and C. L Harold. Organic compound synthes on the primitive earth. Science, 130:251, 1959.

[9] Antonio Lazcano and Jeffrey L Bada. The 1953 Stanley L. Miller experiment: fifty years of prebiotic organic chemistry. Origins of Life and Evolution of the Biosphere, $33(3): 235-242,2003$.

[10] Hugh G. M Hill and Joseph A Nuth. The catalytic potential of cosmic dust: implications for prebiotic chemistry in the solar nebula and other protoplanetary systems. Astrobiology, 3(2):291-304, 2003.

[11] Michael P Callahan, Karen E Smith, H James Cleaves, Josef Ruzicka, Jennifer C Stern, Daniel P Glavin, Christopher H House, and Jason P Dworkin. Carbonaceous meteorites contain a wide range of extraterrestrial nucleobases. Proceedings of the National Academy of Sciences, 108(34):13995-13998, 2011.

[12] Jan M Hollis, Frank J Lovas, and Philip R Jewell. Interstellar glycolaldehyde: the first sugar. The Astrophysical Journal Letters, 540(2):L107, 2000. 
[13] Arnaud Belloche, Holger S. P Müller, Karl M Menten, Peter Schilke, and Claudia Comito. Complex organic molecules in the interstellar medium: IRAM $30 \mathrm{~m}$ line survey of Sagittarius B2 (N) and (M). arXiv preprint arXiv:1308.5062, 2013.

[14] Milton J Rosen and Joy T Kunjappu. Surfactants and interfacial phenomena. John Wiley \& Sons, 2012.

[15] J. W Goodby. Liquid crystals and life. Liquid crystals, 24(1):25-38, 1998.

[16] D. G Dervichian. The control of lyotropic liquid-crystals, biological and medical implications. Molecular Crystals and Liquid Crystals, 40(1):19-31, 1977.

[17] Friedrich Reinitzer. Contributions to the knowledge of cholesterol. Liquid Crystals, $5(1): 7-18,1989$.

[18] Georges Friedel. The mesomorphic states of matter. Ann. Phys, 18:273-474, 1922.

[19] Fritz Vollrath and David P Knight. Liquid crystalline spinning of spider silk. Nature, 410(6828):541-548, 2001.

[20] Ian William Hamley. Liquid crystal phase formation by biopolymers. Soft Matter, 6(9):1863-1871, 2010.

[21] Koji Yonekura, Saori Maki-Yonekura, and Keiichi Namba. Complete atomic model of the bacterial flagellar filament by electron cryomicroscopy. Nature, 424(6949):643-650, 2003.

[22] Sean Pitman. http://www.detectingdesign.com/flagellum.html.

[23] Michael J Behe. Irreducible complexity: Obstacle to Darwinian evolution. Philosophy of Biology: An Anthology, page 427, 2009.

[24] Sergei M Mirkin. DNA topology: fundamentals. eLS, 2001.

[25] X Auvray, C Petipas, R Anthore, I Rico, and A Lattes. X-ray diffraction study of mesophases of cetyltrimethylammonium bromide in water, formamide, and glycerol. The Journal of Physical Chemistry, 93(21):7458-7464, 1989.

[26] Stephen T Hyde. Identification of lyotropic liquid crystalline mesophases. Handbook of applied surface and colloid chemistry, page 299, 2001.

[27] V Luzzati and A Tardieu. Lipid phases: structure and structural transitions. Annual Review of Physical Chemistry, 25(1):79-94, 1974.

[28] Douglas M Fambrough, Dale J Benos, and Richard Epand. Lipid polymorphism and membrane properties, volume 44. 1997.

[29] Ivan I Smalyukh, S. V Shiyanovskii, and O. D Lavrentovich. Three-dimensional imaging of orientational order by fluorescence confocal polarizing microscopy. Chemical Physics Letters, 336(1):88-96, 2001.

[30] O. D Lavrentovich. Fluorescence confocal polarizing microscopy: Three-dimensional imaging of the director. Pramana, 61(2):373-384, 2003.

[31] Michiel Müller. Introduction to confocal fluorescence microscopy, volume 69. SPIE press, 2006. 
[32] A. F Chan, D Fennell Evans, and E. L Cussler. Explaining solubilization kinetics. AIChE Journal, 22(6):1006-1012, 1976.

[33] Alexey Kabalnov and Jeffry Weers. Kinetics of mass transfer in micellar systems: surfactant adsorption, solubilization kinetics, and ripening. Langmuir, 12(14):34423448, 1996.

[34] Alexey S Kabalnov. Can micelles mediate a mass transfer between oil droplets? Langmuir, 10(3):680-684, 1994.

[35] Brendan Joseph Carroll. The kinetics of solubilization of nonpolar oils by nonionic surfactant solutions. Journal of Colloid and Interface Science, 79(1):126-135, 1981.

[36] Alejandro A Peña and Clarence A Miller. Solubilization rates of oils in surfactant solutions and their relationship to mass transport in emulsions. Advances in colloid and interface science, 123:241-257, 2006.

[37] Suwimon Ariyaprakai and Stephanie R Dungan. Contribution of molecular pathways in the micellar solubilization of monodisperse emulsion droplets. Langmuir, 24(7):3061-3069, 2008.

[38] Parthasakha Neogi and James J Munden. Solubilization kinetics of soluble oils by an ionic surfactant. Journal of colloid and interface science, 305(1):202-203, 2007.

[39] Clarence A Miller. Dissolution rates of surfactants. Interfacial processes and molecular aggregation of surfactants. pages 3-24, Springer, 2008.

[40] P. D Todorov, P. A Kralchevsky, N. D Denkov, G Broze, and A Mehreteab. Kinetics of solubilization of $n$-decane and benzene by micellar solutions of sodium dodecyl sulfate. Journal of colloid and interface science, 245(2):371-382, 2002.

[41] J Fundin, Z Yang, A Kelarakis, I. W Hamley, C Price, and C Booth. Solubilization of alkylcyanobiphenyl liquid crystals in aqueous micellar solutions of a diblock copolymer of propylene oxide and ethylene oxide studied using dynamic and static light scattering. The Journal of Physical Chemistry B, 106(45):11728-11736, 2002.

[42] Zhuo Yang, Evangelia Pousia, Frank Heatley, Colin Price, Colin Booth, Valeria Castelletto, and Ian W Hamley. Solubilization of alkylcyanobiphenyls in aqueous micellar solutions of a diblock copolymer of propylene oxide and ethylene oxide. Langmuir, 17(7):2106-2111, 2001.

[43] D Varade, K Aramaki, and C Stubenrauch. Phase diagrams of wateralkyltrimethylammonium bromide systems. Colloids and Surfaces A: Physicochemical and Engineering Aspects, 315(1):205-209, 2008.

[44] Chandrashekara Rajashekara Haramagatti. Structure, dynamics and phase behaviors of cationic micellar solutions. 2006.

[45] A. J Leadbetter, R. M Richardson, and C. N Colling. The structure of a number of nematogens. Le Journal de Physique Colloques, 36(C1):C1-37, 1975.

[46] Calculated using Advanced Chemistry Development (ACD/Labs) Software v11.02 (1994-2013 ACD/Labs). 
[47] Jean-Christophe Loudet, Philippe Barois, and Philippe Poulin. Colloidal ordering from phase separation in a liquid-crystalline continuous phase. Nature, 407(6804):611-613, 2000 .

[48] Ph Poulin and D. A Weitz. Inverted and multiple nematic emulsions. Physical Review E, 57(1):626, 1998.

[49] T. C Lubensky, David Pettey, Nathan Currier, and Holger Stark. Topological defects and interactions in nematic emulsions. Physical Review E, 57(1):610, 1998.

[50] Philippe Poulin, Holger Stark, T. C Lubensky, and D. A Weitz. Novel colloidal interactions in anisotropic fluids. Science, 275(5307):1770-1773, 1997.

[51] Jean-Christophe Loudet and Philippe Poulin. Liquid crystal emulsions. Journal of dispersion science and technology, 23(1-3):143-154, 2002.

[52] Anupam Sengupta, Christian Bahr, and Stephan Herminghaus. Topological microfluidics for flexible micro-cargo concepts. Soft Matter, 2013.

[53] Zhongqiang Yang and Nicholas L Abbott. Spontaneous formation of water droplets at oil- solid interfaces. Langmuir, 26(17):13797-13804, 2010.

[54] P. B Warren and M Buchanan. Kinetics of surfactant dissolution. Current opinion in colloid $\&$ interface science, 6(3):287-293, 2001.

[55] Mark Buchanan, Stefan U Egelhaaf, and Michael E Cates. Dynamics of interface instabilities in nonionic lamellar phases. Langmuir, 16(8):3718-3726, 2000.

[56] Ian W Hamley and Valeria Castelletto. Biological soft materials. Angewandte Chemie International Edition, 46(24):4442-4455, 2007.

[57] Jung-Whan Kim, Hojin Kim, Myoungbae Lee, and Jules J Magda. Interfacial tension of a nematic liquid crystal/water interface with homeotropic surface alignment. Langmuir, 20(19):8110-8113, 2004.

[58] Guillaume Toquer, Ty Phou, Sophie Monge, Antoine Grimaldi, Maurizio Nobili, and Christophe Blanc. Colloidal shape controlled by molecular adsorption at liquid crystal interfaces. The Journal of Physical Chemistry B, 112(14):4157-4160, 2008.

[59] S Riegelman, N. A Allawala, M. K Hrenoff, and L. A Strait. The ultraviolet absorption spectrum as a criterion of the type of solubilization. Journal of Colloid Science, 13(3):208-217, 1958.

[60] J. C Eriksson. NMR [nuclear magnetic resonance] experiments on solubilization in soap micelles. Acta Chem. Scand, 17:1478-1481, 1963.

[61] J. C Eriksson and Gunilla Gillberg. NMR-studies of the solubilization of aromatic compounds in cetyltrimethylammonium bromide solution II. Acta Chem. Scand, 20(8):2019-2027, 1966.

[62] Joy T Kunjappu, P Somasundaran, and N. J Turro. A luminescence quenching study on the localization problem of tris (2, 2'-bipyridyl) ruthenium $(2+)$ in micelles and hemimicelles. Journal of physical chemistry, 94(22):8464-8468, 1990. 
[63] V. K Kelkar, B. S Valaulikar, Joy T Kunjappu, and C Manohar. Aggregation characteristics of laser dye Rhodamine 6G in aqueous surfactant solutions. Photochemistry and Photobiology, 52(4):717-721, 1990.

[64] Joy T Kunjappu. Luminescence behaviour of C120 - Ru (bpy) ${ }_{3}^{2+}$ donor - quencher pair in aqueous solutions. Journal of Photochemistry and Photobiology A: Chemistry, 78(3):237-240, 1994.

[65] V. K Aswal and P. S Goyal. Role of different counterions and size of micelle in concentration dependence micellar structure of ionic surfactants. Chemical physics letters, 368(1):59-65, 2003.

[66] J. V Joshi, V. K Aswal, and P. S Goyal. SANS study of micellar structures on oil solubilization. In AIP Conference Proceedings, volume 989, page 259, 2008.

[67] Takaaki Sato, Durga P Acharya, Masaya Kaneko, Kenji Aramaki, Yashveer Singh, Masahiko Ishitobi, and Hironobu Kunieda. Oil-induced structural change of wormlike micelles in sugar surfactant systems. Journal of dispersion science and technology, 27(5):611-616, 2006.

[68] P. S Goyal. Small angle neutron scattering from micellar solutions. Phase Transitions: A Multinational Journal, 50(1-3):143-176, 1994.

[69] K Kuperkar, L Abezgauz, D Danino, G Verma, P. A Hassan, V. K Aswal, D Varade, and P Bahadur. Viscoelastic micellar water $/ \mathrm{CTAB} / \mathrm{NaNO}_{3}$ solutions: Rheology, SANS and cryo-TEM analysis. Journal of colloid and interface science, 323(2):403-409, 2008.

[70] Malcolm M Cross. Rheology of non-Newtonian fluids: a new flow equation for pseudoplastic systems. Journal of Colloid Science, 20(5):417-437, 1965.

[71] Pierre J Carreau. Rheological equations from molecular network theories. Journal of Rheology, 16:99, 1972.

[72] Naveen K Pokhriyal, Jayant V Joshi, and Prem S Goyal. Viscoelastic behaviour of cetyl trimethyl ammonium bromide/sodium salicylate/water system:: Effect of solubilisation of different polarity oils. Colloids and Surfaces A: Physicochemical and Engineering Aspects, 218(1):201-212, 2003.

[73] Maria Törnblom and Ulf Henriksson. Effect of solubilization of aliphatic hydrocarbons on size and shape of rodlike $C_{16} \mathrm{TABr}$ micelles studied by ${ }^{2} H$ NMR relaxation. The Journal of Physical Chemistry B, 101(31):6028-6035, 1997.

[74] Vivek Agarwal, Mohit Singh, Gary McPherson, Vijay John, and Arijit Bose. Microstructure evolution in aqueous solutions of cetyl trimethylammonium bromide (CTAB) and phenol derivatives. Colloids and Surfaces A: Physicochemical and Engineering Aspects, 281(1):246-253, 2006.

[75] Pasupati Mukerjee. Solubilization in aqueous micellar systems. Solution chemistry of surfactants. pages 153-174, Springer, 1979.

[76] Vania Croce, Terence Cosgrove, Geoff Maitland, Trevor Hughes, and Göran Karlsson. Rheology, cryogenic transmission electron spectroscopy, and small-angle neutron scattering of highly viscoelastic wormlike micellar solutions. Langmuir, 19(20):8536-8541, 2003. 
[77] Jean-François Berret, Denis C Roux, and Grégoire Porte. Isotropic-to-nematic transition in wormlike micelles under shear. Journal de Physique II, 4(8):1261-1279, 1994.

[78] E Cappelaere, J. F Berret, J. P Decruppe, R Cressely, and P Lindner. Rheology, birefringence, and small-angle neutron scattering in a charged micellar system: Evidence of a shear-induced phase transition. Physical Review E, 56(2):1869, 1997.

[79] Daniel Quemada and Claudio Berli. Describing the flow curve of shear-banding fluids through a structural minimal model. arXiv preprint arXiv:0903.0808, 2009.

[80] Itai Cohen, Benny Davidovitch, Andrew B Schofield, Michael P Brenner, and David A Weitz. Slip, yield, and bands in colloidal crystals under oscillatory shear. Physical review letters, 97(21):215502, 2006.

[81] Olivier Diat, Didier Roux, and Frédéric Nallet. Effect of shear on a lyotropic lamellar phase. Journal de physique II, 3(9):1427-1452, 1993.

[82] Montgomery T Shaw and William J MacKnight. Introduction to polymer viscoelasticity. Wiley. com, 2005.

[83] Fumio Oosawa. Polyelectrolytes. Marcel Dekker, 1971.

[84] Gerald S Manning. Limiting laws and counterion condensation in polyelectrolyte solutions I. colligative properties. The Journal of Chemical Physics, 51:924, 1969.

[85] V. K Aswal and P. S Goyal. Counterions in the growth of ionic micelles in aqueous electrolyte solutions: a small-angle neutron scattering study. Physical Review E, 61(3):2947, 2000.

[86] C. L. A Berli, J. A Deiber, and D Quemada. On the viscosity of concentrated suspensions of charged colloids. Latin American applied research, 35(1):15-22, 2005.

[87] M. G Berni, C. J Lawrence, and D Machin. A review of the rheology of the lamellar phase in surfactant systems. Advances in colloid and interface science, 98(2):217-243, 2002 .

[88] P Partal, A. J Kowalski, D Machin, N Kiratzis, M. G Berni, and C. J Lawrence. Rheology and microstructural transitions in the lamellar phase of a cationic surfactant. Langmuir, 17(5):1331-1337, 2001.

[89] Jörg Läuger, Richard Weigel, Klaus Berger, Karl Hiltrop, and Walter Richtering. Rheo-small-angle-light-scattering investigation of shear-induced structural changes in a lyotropic lamellar phase. Journal of colloid and interface science, 181(2):521-529, 1996.

[90] F Cordobés, J. M Franco, and C Gallegos. Rheology of the lamellar liquid-crystalline phase in polyethoxylated alcohol/water/heptane systems. Grasas y aceites, 56(2):96105,2005 .

[91] Stefan Müller, Claus Börschig, Wolfram Gronski, Claudia Schmidt, and Didier Roux. Shear-induced states of orientation of the lamellar phase of $C_{12} E_{4} /$ water. Langmuir, 15(22):7558-7564, 1999. 
[92] Juan Carlos López-Montilla, Paulo Emilio Herrera-Morales, Samir Pandey, and Dinesh O Shah. Spontaneous emulsification: Mechanisms, physicochemical aspects, modeling, and applications. Journal of dispersion science and technology, 23(1-3):219$268,2002$.

[93] A. Z Patashinski, R Orlik, K Paclawski, M. A Ratner, and B. A Grzybowski. The unstable and expanding interface between reacting liquids: Theoretical interpretation of negative surface tension. Soft Matter, 8(5):1601-1608, 2012.

[94] Kevin P Browne, David A Walker, Kyle J. M Bishop, and Bartosz A Grzybowski. Self-division of macroscopic droplets: Partitioning of nanosized cargo into nanoscale micelles. Angewandte Chemie, 122(38):6908-6911, 2010.

[95] Jintao Zhu and Ryan C Hayward. Spontaneous generation of amphiphilic block copolymer micelles with multiple morphologies through interfacial instabilities. Journal of the American Chemical Society, 130(23):7496-7502, 2008.

[96] Shashi Thutupalli. Swimming droplets: Artificial squirmers. In Towards Autonomous Soft Matter Systems, pages 79-94. Springer, 2014.

[97] Alberto Fernández-Nieves, D. R Link, M Marquez, and David A Weitz. Topological changes in bipolar nematic droplets under flow. Physical review letters, 98(8):087801, 2007.

[98] Ch Bahr. Experimental study of prewetting transitions by systematic variation of the surface field at nematic liquid crystal/water interfaces. EPL (Europhysics Letters), 88(4):46001, 2009 .

[99] Xunda Feng and Christian Bahr. Surface order at surfactant-laden interfaces between isotropic liquid crystals and liquid phases with different polarity. Physical Review E, 84(3):031701, 2011.

[100] K. S Chan and D. O Shah. The physico-chemical conditions necessary to produce ultralow interfacial tension at the oil/brine interface. In Surface Phenomena in Enhanced Oil Recovery, pages 53-72. Springer, 1981.

[101] K. S Chan and D. O Shah. The molecular mechanism for achieving ultra low interfacial tension minimum in a petroleum sulfonate/oil/brine system. Journal of Dispersion Science and Technology, 1(1):55-95, 1980.

[102] Clarence A Miller, Rei-Nan Hwan, William J Benton, and Tomlinson Fort Jr. Ultralow interfacial tensions and their relation to phase separation in micellar solutions. Journal of Colloid and Interface Science, 61(3):554-568, 1977.

[103] Igor Muševič. Integrated and topological liquid crystal photonics. Liquid Crystals, 41(3):418-429, 2014.

[104] Matjaž Humar, Miha Ravnik, Stane Pajk, and Igor Muševič. Electrically tunable liquid crystal optical microresonators. Nature Photonics, 3(10):595-600, 2009.

[105] M Humar and I Muševič. 3D microlasers from self-assembled cholesteric liquid-crystal microdroplets. Optics express, 18(26):26995-27003, 2010. 
[106] V. S. R Jampani, M Humar, and I Muševič. Resonant transport of light from planar polymer waveguide into liquid-crystal microcavity. Optics Express, 21(18):2050620516, 2013.

[107] Chang-Hyun Jang, Li-Lin Cheng, Christopher W Olsen, and Nicholas L Abbott. Anchoring of nematic liquid crystals on viruses with different envelope structures. Nano letters, 6(5):1053-1058, 2006.

[108] Jeffrey M Brake and Nicholas L Abbott. Coupling of the orientations of thermotropic liquid crystals to protein binding events at lipid-decorated interfaces. Langmuir, 23(16):8497-8507, 2007.

[109] Jeffrey M Brake, Maren K Daschner, Yan-Yeung Luk, and Nicholas L Abbott. Biomolecular interactions at phospholipid-decorated surfaces of liquid crystals. Science, 302(5653):2094-2097, 2003.

[110] Elvira Tjipto, Katie D Cadwell, John F Quinn, Angus P. R Johnston, Nicholas L Abbott, and Frank Caruso. Tailoring the interfaces between nematic liquid crystal emulsions and aqueous phases via layer-by-layer assembly. Nano letters, 6(10):2243$2248,2006$.

[111] O. D Lavrentovich and Yu A Nastishin. Division of drops of a liquid-crystal in the case of a cholesteric-smectic-A phase-transition. JETP Lett, 40(6):1015-1019, 1984.

[112] O. D Lavrentovich, Yu A Nastishin, V. I Kulishov, Yu S Narkevich, A. S Tolochko, and S. V Shiyanovskii. Helical smectic A. EPL (Europhysics Letters), 13(4):313, 1990.

[113] O. D Lavrentovich and V. V Sergan. Parity-breaking phase transition in tangentially anchored nematic drops. Il Nuovo Cimento D, 12(9):1219-1222, 1990.

[114] Alfred Saupe. Textures, deformations, and structural order of liquid crystals. Journal of Colloid and Interface Science, 58(3):549-558, 1977.

[115] Jürgen-Hinrich Fuhrhop and Jürgen Köning. Binding interactions in micellar and vesicular fibres. 2007.

[116] Rudolf Virchow. Ueber das ausgebreitete vorkommen einer dem nervenmark analogen substanz in den thierischen geweben. Virchows Archiv, 6(4):562-572, 1854.

[117] Ling-Nan Zou and Sidney R Nagel. Stability and growth of single myelin figures. Physical review letters, 96(13):138301, 2006.

[118] Louisa Reissig, David J Fairhurst, Jacques Leng, Michael E Cates, Andrew R Mount, and Stefan U Egelhaaf. Three-dimensional structure and growth of myelins. Langmuir, 26(19):15192-15199, 2010.

[119] M Buchanan, J Arrault, and M. E Cates. Swelling and dissolution of lamellar phases: Role of bilayer organization. Langmuir, 14(26):7371-7377, 1998.

[120] Ling-Nan Zou. Myelin figures: The buckling and flow of wet soap. Physical Review E, 79(6):061502, 2009.

[121] Tino A. A Fonteijn, Dick Hoekstra, and Jan B. F. N Engberts. Vesicle formation of di$n$-alkyl phosphates: Liquid crystalline behavior, myelinization, counterion influence, and stability. Langmuir, 8(10):2437-2447, 1992. 
[122] N Shahidzadeh, D Bonn, and J Meunier. A new mechanism of spontaneous emulsification: Relation to surfactant properties. EPL (Europhysics Letters), 40(4):459, 1997.

[123] Bing-Hung Chen, Clarence A Miller, John M Walsh, Patrick B Warren, J Noel Ruddock, Peter R Garrett, Francoise Argoul, and Christophe Leger. Dissolution rates of pure nonionic surfactants. Langmuir, 16(12):5276-5283, 2000.

[124] M. A Arunagirinathan, Mainak Roy, A. K Dua, C Manohar, and Jayesh R Bellare. Micro-Raman investigations of myelins in aerosol-OT/water system. Langmuir, 20(12):4816-4822, 2004.

[125] Alan P Kennedy, Jonathan Sutcliffe, and Ji-Xin Cheng. Molecular composition and orientation in myelin figures characterized by coherent anti-Stokes Raman scattering microscopy. Langmuir, 21(14):6478-6486, 2005.

[126] W Harbich and W Helfrich. The swelling of egg lecithin in water. Chemistry and physics of lipids, 36(1):39-63, 1984.

[127] Jong Choo Lim and Clarence A Miller. Dynamic behavior and detergency in systems containing nonionic surfactants and mixtures of polar and nonpolar oils. Langmuir, 7(10):2021-2027, 1991.

[128] Veronika Kralj-Iglič, Gregor Gomišček, Janja Majhenc, Vesna Arrigler, and Saša Svetina. Myelin-like protrusions of giant phospholipid vesicles prepared by electroformation. Colloids and Surfaces A: Physicochemical and Engineering Aspects, 181(1):315-318, 2001.

[129] J-R Huang, L-N Zou, and Thomas A Witten. Confined multilamellae prefer cylindrical morphology. The European Physical Journal E, 18(3):279-285, 2005.

[130] Manami Masubuchi, Taro Toyota, Masumi Yamada, and Minoru Seki. Fluidic shearassisted formation of actuating multilamellar lipid tubes using microfabricated nozzle array device. Chemical Communications, 47(29):8433-8435, 2011.

[131] Mayur K Temgire, C Manohar, Jayesh Bellare, and Satyawati S Joshi. Structural studies on nonequilibrium microstructures of dioctyl sodium dodecyl sulfosuccinate (Aerosol-OT) in p-toluenesulfonic acid and phosphatidylcholine. Advances in Physical Chemistry, 2012, 2012.

[132] Lobat Tayebi, Masoud Mozafari, Daryoosh Vashaee, and Atul N Parikh. Structural configuration of myelin figures using fluorescence microscopy. International Journal of Photoenergy, 2012, 2012.

[133] N Anton, J-P Benoit, and P Saulnier. Particular conductive behaviors of emulsion phase inverting. Journal of drug delivery science and technology, 18(2):95-99, 2008.

[134] I Sakurai, T Shibata, M Minobe, and Y Kawamura. Free vertical growth of myelin figures. Molecular Crystals and Liquid Crystals, 363(1):157-165, 2001.

[135] Meiyu Lin, Li Li, Feng Qiu, and Yuliang Yang. Effect of added monovalent electrolytes on the myelin formation from charged lipids. Journal of colloid and interface science, 348(2):505-510, 2010. 
[136] Narges Fathi, Ali-Reza Moradi, Mehdi Habibi, Daryoosh Vashaee, and Lobat Tayebi. Digital holographic microscopy of the myelin figure structural dynamics and the effect of thermal gradient. Biomedical optics express, 4(6):950, 2013.

[137] J-R Huang. Theory of myelin coiling. The European Physical Journal E, 19(4):399412,2006 .

[138] Kiyoshi Mishima, Kenji Fukuda, and Kiyomitsu Suzuki. Double helix formation of phosphatidylcholine myelin figures. Biochimica et Biophysica Acta (BBA)Biomembranes, 1108(1):115-118, 1992.

[139] Vidar Frette, Ilan Tsafrir, Marie-Alice Guedeau-Boudeville, Ludovic Jullien, Daniel Kandel, and Joel Stavans. Coiling of cylindrical membrane stacks with anchored polymers. Physical review letters, 83(12):2465, 1999.

[140] K Mishima and K Yoshiyama. Growth rate of myelin figures of egg-yolk phosphatidylcholine. Biochimica et Biophysica Acta (BBA)-Biomembranes, 904(1):149-153, 1987.

[141] Ilan Tsafrir, Marie-Alice Guedeau-Boudeville, Daniel Kandel, and Joel Stavans. Coiling instability of multilamellar membrane tubes with anchored polymers. Physical Review E, 63(3):031603, 2001.

[142] C. D Santangelo and P Pincus. Coiling instabilities of multilamellar tubes. Physical Review E, 66(6):061501, 2002.

[143] Hitesh Dave, Megha Surve, C Manohar, and Jayesh Bellare. Myelin growth and initial dynamics. Journal of colloid and interface science, 264(1):76-81, 2003.

[144] Joel Stavans. Instabilities of membranes with anchored polymers. Physica A: Statistical Mechanics and its Applications, 306:368-375, 2002.

[145] C-M Chen, C. F Schmidt, P. D Olmsted, and F. C MacKintosh. Instability of myelin tubes under dehydration: Deswelling of layered cylindrical structures. Physical Review E, 64(5):050903, 2001.

[146] Ke-Chun Lin, Robert M Weis, and Harden M McConnell. Induction of helical liposomes by $\mathrm{Ca}^{2+}$-mediated intermembrane binding. 1982 .

[147] Mark Buchanan. Nonlinear dynamics in surfactant systems. ACS SYMPOSIUM SERIES, 869:226-235, 2004.

[148] Mohit Haran, Ashim Chowdhury, C Manohar, and Jayesh Bellare. Myelin growth and coiling. Colloids and Surfaces A: Physicochemical and Engineering Aspects, 205(1):2130, 2002.

[149] Giuseppe Battaglia and Anthony J Ryan. Neuron-like tubular membranes made of diblock copolymer amphiphiles. Angewandte Chemie International Edition, 45(13):2052-2056, 2006.

[150] Jinhua Bai and Clarence A Miller. Experiments and modeling of growth of myelinic figures in Aerosol OT/water system. Colloids and Surfaces A: Physicochemical and Engineering Aspects, 244(1):113-119, 2004.

[151] I Sakurai. Concentration gradient along the long axis of myelin figures of phosphatidylcholine. Biochimica et Biophysica Acta (BBA)-Biomembranes, 815(1):149-152, 1985. 
[152] M. A Arunagirinathan, C Manohar, and Jayesh R Bellare. Eroded myelin figures. Langmuir, 20(11):4318-4321, 2004.

[153] Rajiv Taribagil, M. A Arunagirinathan, C Manohar, and Jayesh R Bellare. Extended time range modeling of myelin growth. Journal of colloid and interface science, 289(1):242-248, 2005.

[154] Xinjiang Chen and Kaoru Tsujii. Synthetic myelin figures immobilized in polymer gels. Soft Matter, 3(7):852-856, 2007.

[155] Christophe Blanc. Interplay between growth mechanisms and elasticity in liquid crystalline nuclei. Progress of Theoretical Physics Supplement, 175:93-102, 2008.

[156] Patrick Oswald and Pawel Pieranski. Nematic and cholesteric liquid crystals: concepts and physical properties illustrated by experiments, volume 1. CRC press, 2006.

[157] Shweta Aggarwal, Larisa Yurlova, and Mikael Simons. Central nervous system myelin: structure, synthesis and assembly. Trends in cell biology, 21(10):585-593, 2011.

[158] J. M Seddon and R. H Templer. Polymorphism of lipid-water systems. Handbook of biological physics, 1:97-160, 1995.

[159] Karthik Peddireddy, Pramoda Kumar, Shashi Thutupalli, Stephan Herminghaus, and Christian Bahr. Solubilization of thermotropic liquid crystal compounds in aqueous surfactant solutions. Langmuir, 28(34):12426-12431, 2012.

[160] Karthik Peddireddy, Pramoda Kumar, Shashi Thutupalli, Stephan Herminghaus, and Christian Bahr. Myelin structures formed by thermotropic smectic liquid crystals. Langmuir, 2013.

[161] H Tajalli, A Ghanadzadeh Gilani, M. S Zakerhamidi, and P Tajalli. The photophysical properties of Nile red and Nile blue in ordered anisotropic media. Dyes and Pigments, 78(1):15-24, 2008.

[162] A Sorrenti, O Illa, and R. M Ortuño. Amphiphiles in aqueous solution: well beyond a soap bubble. Chemical Society Reviews, 42(21):8200-8219, 2013.

[163] Katsuhiro Maeda and Eiji Yashima. Dynamic helical structures: detection and amplification of chirality. Topics in Current Chemistry, 265:47-88, 2006.

[164] E Thomas Pashuck and Samuel I Stupp. Direct observation of morphological tranformation from twisted ribbons into helical ribbons. Journal of the American Chemical Society, 132(26):8819-8821, 2010.

[165] Yevgeniya V Zastavker, Neer Asherie, Aleksey Lomakin, Jayanti Pande, Joanne M Donovan, Joel M Schnur, and George B Benedek. Self-assembly of helical ribbons. Proceedings of the National Academy of Sciences, 96(14):7883-7887, 1999.

[166] Hee-Young Lee, Hyuntaek Oh, Jae-Ho Lee, and Srinivasa R Raghavan. Shedding light on helical microtubules: Real-time observations of microtubule self-assembly by light microscopy. Journal of the American Chemical Society, 134(35):14375-14381, 2012.

[167] Hiroshi Yanagawa, Yoko Ogawa, Hiroyuki Furuta, and Katsushige Tsuno. Spontaneous formation of superhelical strands. Journal of the American Chemical Society, 111(13):4567-4570, 1989. 
[168] E Neuzil, J Fourche, R Jensen, H Jensen, and G Morin. Structural requirements of sterols for myelin tube formation with sodium oleate. Biochimica et Biophysica Acta (BBA)-Biomembranes, 641(1):11-19, 1981.

[169] Inge Zöller, Marion Meixner, Dieter Hartmann, Heinrich Büssow, Rainer Meyer, Volkmar Gieselmann, and Matthias Eckhardt. Absence of 2-hydroxylated sphingolipids is compatible with normal neural development but causes late-onset axon and myelin sheath degeneration. The Journal of Neuroscience, 28(39):9741-9754, 2008.

[170] Paavo J Riekkinen, Jorma Palo, Antti U Arstila, Heikki J Savolainen, Urpo K Rinne, Erkki K Kivalo, and Harry Frey. Protein composition of multiple sclerosis myelin. Archives of neurology, 24(6):545, 1971.

[171] Robert K Yu, Kunihiro Ueno, Gilbert H Glaser, and Wallace W Tourtellotte. Lipid and protein alterations of spinal cord and cord myelin of multiple sclerosis. Journal of neurochemistry, 39(2):464-477, 1982.

[172] Neil M Ribe, Mehdi Habibi, and Daniel Bonn. Liquid rope coiling. Annual Review of Fluid Mechanics, 44:249-266, 2012.

[173] Neil M Ribe, Herbert E Huppert, Mark A Hallworth, Mehdi Habibi, Daniel Bonn, et al. Multiple coexisting states of liquid rope coiling. Journal of fluid mechanics, 555(1):275-297, 2006.

[174] Thomas Cubaud, Bibin M Jose, and Samira Darvishi. Folded micro-threads: Role of viscosity and interfacial tension. Physics of Fluids, 23:042002, 2011.

[175] L Mahadevan, William S Ryu, and Aravinthan D. T Samuel. Fluid 'rope trick' investigated. Nature, 392(6672):140-140, 1998.

[176] Kerry J Vahala. Optical microcavities. Nature, 424(6950):839-846, 2003.

[177] A Kiraz, Y Karadag, and A. F Coskun. Spectral tuning of liquid microdroplets standing on a superhydrophobic surface using electrowetting. Applied Physics Letters, 92(19):191104-191104, 2008.

[178] Mitsunori Saito, Hiroya Shimatani, and Hideyuki Naruhashi. Tunable whispering gallery mode emission from a microdroplet in elastomer. Optics Express, 16(16):1191511919, 2008.

[179] C. R Lee, S. H Lin, C. H Guo, S. H Chang, T. S Mo, and S. C Chu. All-optically controllable random laser based on a dye-doped polymer-dispersed liquid crystal with nano-sized droplets. Opt. Express, 18(3):2406-2412, 2010.

[180] Damian J Gardiner, Stephen M Morris, Philip J. W Hands, Carrie Mowatt, Rupert Rutledge, Timothy D Wilkinson, and Harry J Coles. Paintable band-edge liquid crystal lasers. Opt. Express, 19:2432-2439, 2011.

[181] Gabriella Cipparrone, Alfredo Mazzulla, Alfredo Pane, Raul Josue Hernandez, and Roberto Bartolino. Chiral self-assembled solid microspheres: A novel multifunctional microphotonic device. Advanced Materials, 23(48):5773-5778, 2011.

[182] M Humar and I Muševič. Surfactant sensing based on whispering-gallery-mode lasing in liquid-crystal microdroplets. Optics express, 19(21):19836-19844, 2011. 
[183] Hiroyoshi Naito, Masahiro Okuda, and Ou-Yang Zhong-Can. Pattern formation and instability of smectic-A filaments grown from an isotropic phase. Physical Review E, 55(2):1655, 1997.

[184] Masayoshi Todorokihara, Yosuke Iwata, and Hiroyoshi Naito. Periodic buckling of smectic-A tubular filaments in an isotropic phase. Physical Review E, 70(2):021701, 2004 .

[185] A Adamczyk. Droplet-filament-membrane-vesicle-double vesicle spontaneous transformations in nematosmectogen NPOOB/silicone oil systems. Supramolecular Science, 4(3):179-183, 1997.

[186] A Adamczyk. Phase transitions in liquid crystal filamentary structures. Molecular Crystals and Liquid Crystals, 351(1):145-152, 2000.

[187] A Adamczyk. Phase transitions in freely suspended smectic droplets. cotton-mouton technique, architecture of droplets and formation of nematoids. Molecular Crystals and Liquid Crystals, 170(1):53-69, 1989.

[188] E Weinan and Peter Palffy-Muhoray. Dynamics of filaments during the isotropicsmectic-A phase transition. Journal of Nonlinear Science, 9(4):417-437, 1999.

[189] Masayoshi Todorokihara, Koki Fujihara, and Hiroyoshi Naito. Helical tubular filaments of a smectic-A phase in an isotropic phase. Molecular Crystals and Liquid Crystals, 412(1):77-83, 2004.

[190] Etienne Brasselet, Naoki Murazawa, Hiroaki Misawa, and Saulius Juodkazis. Optical vortices from liquid crystal droplets. Physical review letters, 103(10):103903, 2009.

[191] Kerry Vahala. Optical microcavities. World Scientific, 2004.

[192] S. M Spillane, T. J Kippenberg, O. J Painter, and K. J Vahala. Ideality in a fiber-tapercoupled microresonator system for application to cavity quantum electrodynamics. Physical Review Letters, 91(4):043902, 2003.

[193] Karthik Peddireddy, VSR Jampani, Shashi Thutupalli, Stephan Herminghaus, Christian Bahr, and Igor Muševič. Lasing and waveguiding in smectic a liquid crystal optical fibers. Optics Express, 21(25):30233-30242, 2013.

[194] Matjaž Humar. Liquid-crystal microdroplets as optical microresonators and lasers. PhD thesis, Institute Jozef Stefan, 2012.

[195] Van Duong Ta, Rui Chen, Lin Ma, Yong Jun Ying, and Han Dong Sun. Whispering gallery mode microlasers and refractive index sensing based on single polymer fiber. Laser \& Photonics Reviews, 7(1):133-139, 2013.

[196] Harry Coles and Stephen Morris. Liquid-crystal lasers. Nature Photonics, 4(10):676$685,2010$.

[197] F Vandenbrouck, S Bardon, M. P Valignat, and A. M Cazabat. Wetting transition and divergence of the extrapolation length near the nematic-isotropic transition. Physical review letters, 81(3):610, 1998. 


\section{Index of Figures}

1 Cosmological structures that contain potentially life-enabling molecules . . . 2

2 Molecular structures of typical organic molecules that form LC phases . . . . 3

3 Schematic representation of typical calamitic and discotic LC molecules . . . 4

4 Schematic illustration of the molecular order in a nematic LC . . . . . . . . 5

5 Schematic representation of the thermotropic LC phases with positional order 5

6 Schematic representation of the thermotropic chiral LC phases . . . . . . . 6

$7 \quad$ Cholesteric droplets in aqueous micellar solutions . . . . . . . . . . . . 8

8 A typical phase diagram of an amphiphile-water system . . . . . . . . . . 9

9 Schematic view of the polarizing optical microscope . . . . . . . . . . . 15

10 Schematic view of the fluorescence confocal polarization microscopy . . . . . 16

11 Schematic diagram of the optical setup for lasing and waveguiding . . . . . . 18

12 Photo of the actual optical setup . . . . . . . . . . . . . 18

13 Phase diagrams of NLCs in TTAB 25 wt\% aqueous solutions . . . . . . . . 22

14 Visual appearances of equilibrium states of liquid crystals in TTAB 25 wt\%

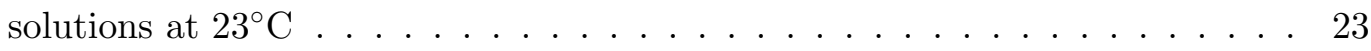

15 Optical microscopy images of LC-TTAB $25 \mathrm{wt} \%$ equilibrium solutions at $23^{\circ} \mathrm{C} 24$

16 Aqueous daughter droplets in a $5 \mathrm{CB}$ parent droplet . . . . . . . . . . . . . 25

17 Rate of dissolution vs initial droplet size . . . . . . . . . . . . . . . . . 27

18 Effect of temperature on 5CB droplet solubilization rates . . . . . . . . 28

19 Chain length dependence on solubilization kinetics . . . . . . . . . . . . . . 29

20 Solubilization process of MBBA in TTAB solution . . . . . . . . . . . . 30

21 Effect of surfactant concentration on threshold temperature . . . . . . . . . . 31

22 Solubilization rate of MBBA droplets vs Temperature . . . . . . . . . . . . . . 32

23 Rate of solubulization of LC droplets vs TTAB concentration . . . . . . . . . 33

24 Schematic diagram of plausible oil solubilization pathways . . . . . . . . . 35

25 Model fittings for LC solubilization in TTAB solutions . . . . . . . . . . . . . 37

26 Possible loci of solubilization . . . . . . . . . . . . . . . . . . 39

27 Steady shear flow curves of 5CB in TTAB 25 wt\% solutions . . . . . . . . . 39

28 Comparing models for a steady shear flow curve of 5CB in TTAB 25 wt\%

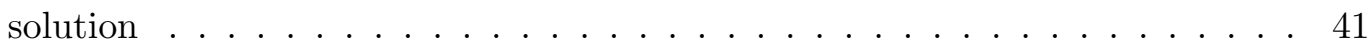

29 Shear banding phenomenon in 5CB-TTAB 25 wt\% systems . . . . . . . . . 43

30 Effect of the molecular chain length of solubilizate on viscosity values . . . . 44

31 Oscillatory shear flow curves of 5CB in TTAB 25 wt\% solutions. . . . . . . . 45

32 Steady shear flow curves of $L_{1}$ phase MBBA-TTAB 25 wt\% solutions at $23^{\circ} \mathrm{C} \quad 46$

33 Steady shear flow curves of lamellar phase MBBA-TTAB $25 \mathrm{wt} \%$ solutions

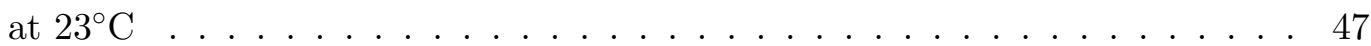

34 SANS spectra for $5 \mathrm{CB}$-TTAB $25 \mathrm{wt} \%$ systems with varying $5 \mathrm{CB}$ concentration 49 
35 Nematic director profile in a self-propelled 5 CB droplet . . . . . . . . . . . 50

36 The equilibrium velocities of self-propelled 5CB droplets vs surfactant concentration at $23^{\circ} \mathrm{C} \ldots \ldots \ldots \ldots \ldots \ldots \ldots$

37 Head-on collision between two self-propelling 5CB droplets in a square capillary 52

38 Interfacial instability in a large nematic $8 \mathrm{CB}$ droplet . . . . . . . . . . . . 54

39 Reversible transformation between an isotropic droplet and a nematic filament 56

40 Effect of temperature on the surface area of a nematic filament . . . . . . . 57

41 Observed structural distortions of a radial $8 \mathrm{CB}$ nematic droplet with a hedgehog defect at the center . . . . . . . . . . . . . . 5 58

42 Average smectic droplet size vs monoolein concentration . . . . . . . . . . . 59

43 Instability at a lipid - water interface . . . . . . . . . . . . . 62

44 Employed methods for investigating filaments' formation and growth . . . . . 64

45 Polarizing microscopy images of smectic-A filaments . . . . . . . . . . . 65

46 FCPM images of a smectic-A 8CB (doped with nile red) filament . . . . . . 66

47 Volume data for a growing $8 \mathrm{CB}$ filament in a square capillary . . . . . . . . . . 67

48 Optical micrographs of an $8 \mathrm{cb}$ sandwiched droplet . . . . . . . . . . . . 68

49 Water transport into a confined 8CB smectic filament . . . . . . . . . . 69

50 Eleven-months-old partially ordered smectic-A filament . . . . . . . . . . . 69

51 Comparison between smectic-A and smectic- $\mathrm{C}^{*}$ filaments' structures . . . . . 70

52 Crossed polarizer images of a C7 filament at different temperatures . . . . . . 71

53 Optical micrographs of double helical myelin figures formed at lipid-aqueous interface . . . . . . . . . . . . . . . . . . . 72

54 Optical micrographs of various structures formed by thermotropic smectic-A filaments . . . . . . . . . . . . . . . . . . . . 73

55 Optical micrographs of various thermotropic smectic-C filaments . . . . . . . 74

56 Optical micrographs of the observed structures in a smectic-C ${ }^{*}$ LC-water system 74

57 Sandwich method - time evolution of the 8CB-aqueous interface . . . . . . . . 75

58 Sandwich method - effect of CTAB concentration on the growth behavior of smectic-A filaments . . . . . . . . . . . . . . . . 76

59 Sandwich method - effect of temperature on growth rates of smectic filaments 77

60 Sandwich method - effect of confining walls on the morphology of smectic filaments . . . . . . . . . . . . . . . . . . . . 78

61 Sandwich method - effect of surfactant chain length on the growth rates of smectic-A filaments . . . . . . . . . . . . . . . . . 79

62 Sandwich method - instabilities in smectic-A filaments . . . . . . . . . . 80

63 Capillary method - growth behavior of smectic-A filaments in different sizes of capillaries . . . . . . . . . . . . . . . . . . . . 8 81

64 Comparison between sandwich and capillary methods . . . . . . . . . . 82

65 LC ropes - surface instability on thermotropic and lyotropic filaments . . . . 83

66 Destabilization of LC-aqueous interface using different methods . . . . . . . . 84

67 Self-healing lyotropic myelin figure . . . . . . . . . . . . . . . . 85

68 Sandwich method - effect of temperature on the stability of 8CB-aqueous interface . . . . . . . . . . . . . . . . . 86

69 Typical coiling structures observed in LC-aqueous systems . . . . . . . . . . . 88

70 LC rope to vesicle transformation . . . . . . . . . . . . . . . 88

71 BOAS structure - effect of salt on the LC rope helical coil structure . . . . . 89

72 UV photo-polymerization of smectic-A filaments . . . . . . . . . . . . . 90

73 Self-propelling motion of an 8CB smectic-A droplet . . . . . . . . . . . . . . 91

74 Production of the desired structures by manipulating smectic filaments . . . . 92 
75 Light guiding in smectic-A filaments . . . . . . . . . . . . . . . . . . 94

76 Possible regions on the surface of an $8 \mathrm{CB}$ smectic-A filament through which light can be guided . . . . . . . . . . . . . . . . . . 95

77 Light guiding in smectic-A super structures . . . . . . . . . . . . . . 96

78 Measurement of the quality of light guiding in a smectic-A filament . . . . . . 97

79 Measurements of the emitted fluorescence intensities in a smectic-A filament . 97

80 Lasing in Nile red dye doped 8CB smectic-A filaments . . . . . . . . . . . 98

81 Effect of the pulsed laser polarization on lasing threshold values . . . . . . . . 100

82 Coupled WGM cavities (8CB filaments and droplets) . . . . . . . . . . . . 101

83 Circulation of fluorescent light in a smectic- $\mathrm{C}^{*}$ droplet . . . . . . . . . . . . . 102

84 Light guiding through $\mathrm{C} 7$ smectic- $\mathrm{C}^{*}$ filaments . . . . . . . . . . . . . . . 104 


\section{Index of Tables}

1 Phase transition temperatures of LCs . . . . . . . . . . . . . . . . . . . 11

2 Chiral dopants ........................... 12

3 Critical micellar concentration values of amphiphiles . . . . . . . . . . 13

4 Carreau model fitting parameters for 5CB in TTAB 25 wt $\%$ solutions . . . . 41 


\section{Abbreviations}

\begin{tabular}{|c|c|c|}
\hline $5 \mathrm{CB}$ & $=$ & 4-Pentyl-4'-cyanobiphenyl \\
\hline $8 \mathrm{CB}$ & $=$ & $4^{\prime}$-Octyl-4-cyanobiphenyl \\
\hline 8.0 .6 & $=$ & 4-(Hexyloxy)phenyl 4'-(octyloxy)benzoate \\
\hline $8 \mathrm{OPhPy} 8$ & $=$ & 5-Octyl-2-(4-octyloxyphenyl)pyrimidine \\
\hline $\mathrm{AOD}$ & $=$ & Acousto-optic deflector \\
\hline BOAS & $=$ & Beads-on-a-string \\
\hline $\mathrm{C} 7$ & $=$ & 4-(2S,3S)-(2-Chloro-3-methylpentanoyloxy)-4'-heptyloxybiphenyl \\
\hline $\mathrm{C}_{n} \mathrm{E}_{n}$ & $=$ & Polyoxyethylene surfactant \\
\hline CFM & $=$ & Confocal fluorescence microscopy \\
\hline CLC & $=$ & Cholesteric liquid crystal \\
\hline CMC & $=$ & Critical micellar concentration \\
\hline CMOS & $=$ & Complementary metal-oxide semiconductor \\
\hline CTAB & $=$ & Hexadecyltrimethylammonium bromide \\
\hline $\mathrm{C}_{n} \mathrm{TAB}$ & $=$ & n - alkyltrimethylammonium bromide \\
\hline $\mathrm{CW}$ & $=$ & Continuous wave \\
\hline $\mathrm{ECF}$ & $=$ & Extra-cellular fluid \\
\hline Egg-PC & $=$ & Egg-yolk phosphatidylcholine \\
\hline EDL & $=$ & Electrical double layer \\
\hline $\mathrm{Eq}$ & $=$ & Equation \\
\hline FCPM & $=$ & Florescence confocal polarizing microscopy \\
\hline Fig & $=$ & Figure \\
\hline FSR & $=$ & Free spectral range \\
\hline IR & $=$ & Infrared \\
\hline $\mathrm{LC}$ & $=$ & Liquid crystal or liquid crystalline \\
\hline MBBA & $=$ & N-(4-Methoxybenzylidene)-4-butylaniline \\
\hline MLV & $=$ & Multilamellar vesicle \\
\hline $\mathrm{N}\left(\mathrm{N}^{*}\right)$ & $=$ & Nematic (chiral nematic) phase \\
\hline NLC & $=$ & Nematic liquid crystal \\
\hline PBG & $=$ & Photonic band gap \\
\hline PDMS & $=$ & Polydimethylsiloxane \\
\hline PMT & $=$ & Photomultiplier tube \\
\hline SANS & $=$ & Small-angle neutron scattering \\
\hline SDS & $=$ & Sodium dodecyl sulfate \\
\hline $\mathrm{Sm}-\mathrm{A}\left(\mathrm{C}, \mathrm{C}^{*}\right)$ & $=$ & Smectic-A $\left(\mathrm{C}, \mathrm{C}^{*}\right)$ \\
\hline TIR & $=$ & Total internal reflection \\
\hline TSLC & $=$ & Thermotropic smectic liquid crystal \\
\hline TTAB & $=$ & Tetradecyltrimethylammonium bromide \\
\hline UV & $=$ & Ultra violet \\
\hline WGM & $=$ & Whispering-gallery mode \\
\hline $\mathrm{Wt} \%$ & $=$ & Weight percent \\
\hline $3 \mathrm{D}$ & $=$ & Three-dimensional \\
\hline "I" and "Iso" & $=$ & Isotropic \\
\hline
\end{tabular}




\section{Appendix:}

\section{List of publications related to doctoral dissertation}

1. K. Peddireddy, P. Kumar, S. Thutupalli, S. Herminghaus, and Ch. Bahr, "Solubilization of Thermotropic Liquid Crystal Compounds in Aqueous Surfactant Solutions", Langmuir, vol. $28,12426,2012$.

2. K. Peddireddy, P. Kumar, S. Thutupalli, S. Herminghaus, and Ch. Bahr, "Myelin Structures Formed by Thermotropic Smectic Liquid Crystals", Langmuir, vol. 29, 15682, 2013.

3. K. Peddireddy, V. S. R. Jampani, S. Thutupalli, S. Herminghaus, Ch. Bahr, and I. Muševič, "Lasing and waveguiding in smectic A liquid crystal optical fibers", Opt. Express, vol. 21, 30233, 2013. Highlighted in OSA spotlight Vol. 21, 25 (2013), Nat. Photonics, Research Highlights, 8, 84 (2014). 


\section{Curriculum Vitae}

\section{Personal Details}

Name : $\quad$ Karthik Reddy Peddireddy

Date of birth : $\quad 13.06 .1986$

Place of birth : $\quad$ Nagarkurnool, Telangana, India

Address : $\quad$ Philipp-Reis-Straße 9, 37075, Göttingen

Nationality : Indian

\section{Education}

2010 - present:

Doctoral research,

Max Planck Institute for Dynamics and Self Organization, Göttingen, Germany.

Supervisors: Dr. Christian Bahr \&

Prof. Dr. Stephan Herminghaus.

$2008-2009:$

Master of Engineering in Chemical Engineering and Colloids,Polymers and Surfaces,

Carnegie Mellon University, Pittsburgh, USA.

Supervisor: Prof. Dr. Stephen Garoff

$2004-2008:$

Bachelor of Technology in Chemical Engineering, Indian Institute of Technology, Guwahati, India.

Supervisor: Prof. Dr. A. K. Ghosal. 


\section{Erklärung}

1. Ich habe diese Arbeit selbständig und ohne unerlaubte Hilfe angefertigt und dabei nur die angegebenen Quellen benutzt.

2. Eine entsprechende Promotion wurde an keiner anderen Hochschule im In- oder Ausland beantragt; die eingereichte Dissertation oder Teile von ihr wurden nicht für ein anderes Promotionsvorhaben verwendet.

Göttingen, den 19. 03. 2014

Karthik Reddy Peddireddy 

